\title{
MODEL BASED DECOMPOSITION OF MUAPS INTO THEIR CONSTITUENT SFEAPS
}

MARIA GEORGIOU XYDA

A submission presented in partial fulfilment of the requirements of the University of Glamorgan/Prifysgol Morgannwg for the degree of Master of Philosophy

This research programme was carried out in collaboration with the Department of Computer Science, University of Cyprus and the Departments of Neurophysiology and Computational Intelligence, Cyprus Institute of Neurology and Genetics

November 2006 


\section{ABSTRACT}

The motor unit action potential (MUAP) represents the spatial and temporal summation of single fibre extracellular action potentials (SFEAPS) generated from the same motor unit. MUAP morphology is affected by structural reorganisation of the motor unit that takes place due to disorders affecting peripheral nerve and muscle. MUAP features extracted in the time domain like duration, amplitude and phases are extensively used by the neurophysiologist for the assessment of neuromuscular disorders. In this study, a model based decomposition of MUAPS into their constituent SFEAPS is investigated. The aim of this study has been to develop a system that will give to the neurophysiologist a visualization of an "estimated" structural organisation of the motor unit, which includes information about number of fibres, fibre distribution and positioning and fibre diameter. This consists of the inverse problem in EMG.

The mathematical model developed in two dimensions by Dimitrova and Dimitrov (Dimitrova 1974; Dimitrov 1987) was used to generate SFEAPS. In addition this model was extended to three dimensions. Two-dimensional and three-dimensional models of normal MUAPS consisting of 1, 4, 5, 10 and 50 fibres respectively were developed and decomposed for a small recoding radius up to $5 \mathrm{~mm}$. Also three-dimensional models were developed and decomposed for myopathy in cases of variability in diameter, loss of fibres and reinnervation. The non linear least squares optimization procedure based on the Levenberg-Marquardt algorithm was used to obtain a solution to the MUAP decomposition problem i.e. fibre distribution, positioning and diameter. Using this method, a satisfactory solution to the decomposition problem was obtained. Future work will investigate the usefulness of the proposed analysis on MUAPS recorded from normal subjects and subjects suffering with neuromuscular disorders. 


\section{ACKNOWLEDGMENTS}

This work was carried out under the supervision of Costantinos S. Pattichis, Associate Professor with the Department of Computer Science at the University of Cyprus, to whom the Author would like to express gratitude for his support and advice.

In addition, the Author would like to express my sincere thanks to Paul Angel, Professor with the Department of Computer Studies at the Glamorgan University, for his guidance during the course of this thesis.

Furthermore, the Author would like to thank Prof Dimitrova and Prof Dimitrov from The Bulgarian Academy of Sciences for their help at the early stages of her work in selecting and implementing the SFEAP model. 


\section{DECLARATION}

This is to certify that neither this thesis, nor any part of it, has been presented or is being concurrently submitted in candidature for any other University degrees.

\section{Candidate}




\section{CERTIFICATE OF RESEARCH}

This is to certify that, except where specific reference is made, the work described in this thesis is the result of the investigation of the candidate.

\section{Candidate}




\section{CONTENTS}

Page

1. INTRODUCTION 1

1.1 Motor Unit Action Potential 2

1.2 Aim 3

1.3 Objectives 4

1.4 Guide to Thesis Contents 4

2. EXTRACELLULAR POTENTIAL MODELLING AND 5

MUAP SIGNAL ANALYSIS

2.1 The Motor Unit 5

$\begin{array}{ll}\text { 2.2 Single Fibre Action Potential Modelling } & 6\end{array}$

2.2.1 Core-Conduction Model (Weerd 1984) 8

2.2.1.1 Model Details $\quad 8$

2.2.1.2 Limitations 99

Volume Conduction as a Filter Process (Greco, Clark and 9

Harman,1977)

2.2.2.1 Model Details

$\begin{array}{ll}\text { 2.2.2.2 Limitations } & 16\end{array}$

$\begin{array}{ll}\text { 2.2.3 Dipole Model (Boyd and Lawrence, 1978) } & 17\end{array}$

$\begin{array}{ll}\text { 2.2.3.1 Model Details } & 17\end{array}$

$\begin{array}{ll}\text { 2.2.3.2 Limitations } & 18\end{array}$

2.2.4 Dipole Model (Dimitrova 1974; Dimitrov 1987) 18

$\begin{array}{ll}\text { 2.2.4.1 Model Details } & 18\end{array}$

$\begin{array}{ll}\text { 2.2.4.2 Limitations } & 19\end{array}$

2.2.5 Tripole Model (Rosenfalck, 1969) 20

$\begin{array}{ll}\text { 2.2.5.1 Model Details } & 20\end{array}$ 
3.4 Description of the Experiments 36

3.4.1 Simulation and decomposition of a two-dimensional model

3.4.3 Simulation and decomposition of a three-dimensional model in the case of a myopathy

\section{RESULTS}

4.1 MUAP Simulation and Decomposition Using a Two-Dimensional Model

4.1.3 MUAP simulation for 5 fibres 44

4.1.4 MUAP simulation for 10 fibres 46

4.1.5 Summarized results for MUAP simulation using a $\quad 48$ two-dimensional model 
4.2.1 MUAP simulation 1: 50 fibres, number of iterations 251, Error $=8.6774$

4.2.2 MUAP simulation 2:50 fibres, number of iterations 1011, Error $=0.0187$

4.2.3 MUAP simulation 3:50 fibres, number of iterations 5065, Error $=2.7241 \mathrm{e}-008$

4.2.4 Summarized results for normal MUAP simulation using a three-dimensional model

4.3 MUAP Simulation and Decomposition using a Three-Dimensional

Model for Myopathy

4.3.1 MUAP myopathy simulation 1, loss of half of the fibres: number of iterations 221, Error $=0.3823$

4.3.2 MUAP myopathy simulation 2, loss of half of the fibres: number of iterations 1115, Error=2.0846e-005

4.3.3 MUAP myopathy simulation 3, loss of half of the fibres: number of iterations 1113 , Error $=3.8031 \mathrm{e}-007$

4.3.4 MUAP myopathy simulation 4, variability in diameter: number of iterations 251, Error $=346.1319$

4.3.5 MUAP myopathy simulation 5, variability in diameter: number of iterations 1011, Error $=5.9174$

4.3.6 MUAP myopathy simulation 6, variability in diameter: number of iterations 5066, Error $=4.1195 \mathrm{e}-004$

4.3.7 MUAP myopathy simulation 7 , reinnervation of 25 fibres in both planes of the MU territory: number of iterations 376, Error=6.3581

4.3.8 MUAP myopathy simulation 8 , reinnervation of 25 fibres in both planes of the MU territory: number of iterations 1132,Error=0.0087

4.3.9 MUAP myopathy simulation 9, reinnervation of 25 fibres in both 66 planes of the MU territory: number of iterations 5294, Error $=1.7908 \mathrm{e}-007$

4.3.10 MUAP myopathy simulation 10, reinnervation of 25 fibres in the 68 right plane of the MU territory: number of iterations 376 , Error $=3.4393$ 
4.3.11 MUAP myopathy simulation 11, reinnervation of 25 fibres in the right plane of the MU territory: number of iterations 1113, Error $=0.0012$

4.3.12 MUAP myopathy simulation 12, reinnervation of 25 fibres in the right plane of the MU territory: number of iterations 5295, Error $=7.8821 \mathrm{e}-009$

4.3.13 Summarized results for myopathy MUAP simulation using a threedimensional model

5.1 MUAP Simulation and Decomposition Using a Two-dimensional Model for Normal MUAPS

5.2 MUAP Simulation and Decomposition Using a Three-Dimensional Model for Normal MUAPS

5.3 MUAP Simulation and Decomposition Using a Three-Dimensional Model for Myopathy MUAPS

6. CONCLUSIONS AND FUTURE WORK

6.1 Conclusions

6.2 Future Work

7. REFERENCES

APPENDIX 1

MATLAB FUNCTIONS FOR TWO-DIMENSIONAL MODELS

APPENDIX 2

MATLAB FUNCTIONS FOR THREE-DIMENSIONAL MODELS

APPENDIX 3

TABLES WITH SIMULATED NORMAL MUAPS FOR

THREE-DIMENSIONAL MODELS

APPENDIX 4

TABLES WITH SIMULATED MYOPATHY MUAPS FOR

THREE-DIMENSIONAL MODELS 


\section{LIST OF FIGURES}

Fig. 1.1 Single Fibre Extracellular Action Potential (EAP).

Fig. 2.1 Motor Unit

Fig. 2.2 Representation of the core-conduction model as an electric analog

Fig. 2.3 Filter process.

Fig. 2.4 Mathematical implementation of the filter process.

Fig. 2.5 The volume conduction model.

Fig. 2.6 The dipole model.

Fig. 2.7 The tripole model.

Fig. 2.8 The line source model.

Fig. 4.1 Simulation and decomposition of 1 fibre for a test radius of $0.6 \mathrm{~mm}$.

Fig. 4.2 Simulation and decomposition of 4 fibres for a test radius of $0.9 \mathrm{~mm}$.

Fig. 4.3 Simulation and decomposition of 5 fibres for a test radius of $1.0 \mathrm{~mm}$.

Fig. 4.4 Simulation and decomposition of 10 fibres for a test radius of $1.2 \mathrm{~mm}$.

Fig. 4.5 Simulation and decomposition of 50 fibres for a test radius of $5 \mathrm{~mm}$. No of iterations $=251$.

Fig. 4.6 Simulation and decomposition of 50 fibres for a test radius of $5 \mathrm{~mm}$. No of iterations $=1011$.

Fig. 4.7 Simulation and decomposition of 50 fibres for a test radius of $5 \mathrm{~mm}$. No of 53 iterations $=5065$. 
Fig. 4.8 Loss of half of the fibres. Simulation and decomposition of 25 fibres for a 56 test of radius $5 \mathrm{~mm}$. No of iterations $=221$.

Fig. 4.9 Loss of half of the fibres. Simulation and decomposition of 25 fibres for a 57 test radius of $5 \mathrm{~mm}$. No of Iterations $=1115$.

Fig. 4.10 Loss of half of the fibres. Simulation and decomposition of 25 fibres for a 59 test radius of $5 \mathrm{~mm}$. No of iterations $=1113$.

Fig. 4.11 Variability in diameter. Simulation and decomposition of 50 fibres for a test radius of $5 \mathrm{~mm}$. No of iterations $=251$.

Fig. 4.12 Variability in diameter. Simulation and decomposition of 50 fibres for a test radius of $5 \mathrm{~mm}$. No of iterations $=1011$.

Fig. 4.13 Variability in diameter. Simulation and decomposition of 50 fibres for a test radius $5 \mathrm{~mm}$. No of iterations $=5066$.

Fig. 4.14 Reinnervation of fibres. Simulation and decomposition of 75 fibres for a 64 test radius of $5 \mathrm{~mm}$. Twenty five fibres were reinnervated in both planes of the MU territory. No of Iterations $=376$.

Fig. 4.15 Reinnervation of fibres. Simulation and decomposition of 75 fibres for a test radius of $5 \mathrm{~mm}$. Twenty five fibres were reinnervated in both planes of the MU territory. No of iterations $=1132$.

Fig. 4.16 Reinnervation of fibres. Simulation and decomposition of 75 fibres for a 67 test radius of $5 \mathrm{~mm}$. Twenty five fibres were reinnervated in both planes of the MU territory. No of Iterations $=5294$.

Fig. 4.17 Reinnervation of fibres. Simulation and decomposition of 75 fibres for a 68 test radius of $5 \mathrm{~mm}$. Twenty five fibres were reinnervated in the right plane of the MU territory. No of iterations $=376$.

Fig. 4.18 Reinnervation of fibres. Simulation and decomposition of 75 fibres for a test radius of $5 \mathrm{~mm}$. Twenty five fibres were reinnervated in the right plane of the MU territory. No of iterations $=1133$.

Fig. 4.19 Reinnervation of fibres. Simulation and decomposition of 75 fibres for a test radius of $5 \mathrm{~mm}$. Twenty five fibres were reinnervated in the right plane of the MU territory. No of iterations $=5295$. 


\section{LIST OF TABLES}

Table 4.1 MUAP simulation and decomposition for 1 fibre.

Table 4.2 MUAP simulation and decomposition for 4 fibres.

Table 4.3 MUAP simulation and decomposition for 5 fibres.

Table 4.4 MUAP simulation and decomposition for 10 fibres.

Table 4.5 Summarized results for two-dimensional models.

Table 4.6 Normal MUAP, summarized results for three-dimensional models.

Table 4.7 Summarized results for myopathic MUAPS using a three-dimensional model.

Table A.3.1 Normal MUAP - Simulated values for 50 fibres.

Table A.3.2 Normal MUAP - Initial values for 50 fibres.

Table A.3.3 Normal MUAP - Results for 50 fibres after 251 iterations.

Table A.3.4 Normal MUAP - Results for 50 fibres after 1011 iterations.

Table A.3.5 Normal MUAP - Results for 50 fibres after 5065 iterations.

Table A.4.1 Myopathy MUAP - Loss of the half fibres, simulated values.

Table A.4.2 Myopathy MUAP - Loss of the half fibres, initial values.

Table A.4.3 Myopathy MUAP - loss of the half fibres, results after $221 \quad 116$ iterations.

Table A.4.4 Myopathy MUAP - loss of the half fibres, results after 1115 iterations.

Table A.4.5 Myopathy MUAP - loss of the half fibres, results after 1113 iterations.

Table A.4.6 Myopathy MUAP - diameter variability, simulated values. 
Table A.4.7 Myopathy MUAP - diameter variability, results after 251 iterations.

Table A.4.8 Myopathy MUAP - diameter variability, results after 1011 iterations.

Table A.4.9 Myopathy MUAP - diameter variability, results after 5066 iterations.

Table A.4.10 Myopathy MUAP - reinnervation of 25 fibres in both planes of the MU, simulated values.

Table A.4.11 Myopathy MUAP - reinnervation of 25 fibres in both planes of the MU, initial values.

Table A.4.12 Myopathy MUAP - reinnervation of 25 fibres in both planes of the MU, results after 376 iterations.

Table A.4.13 Myopathy MUAP - reinnervation of 25 fibres in both planes of the MU, results after 1132 iterations.

Table A.4.14 Myopathy MUAP - reinnervation of 25 fibres in both planes of the MU, results after 5294 iterations.

Table A4.15. Myopathic MUAP - reinnervation of 25 fibres in the right plane of the MU, simulated values.

Table A.4.16 Myopathy MUAP - reinnervation of 25 fibres in the right plane of the MU, initial values.

Table A.4.17 Myopathy MUAP - reinnervation of 25 fibres in the right plane of the MU, results after 376 iterations.

Table A.4.18 Myopathy MUAP - reinnervation of 25 fibres in the right plane of the MU, results after 1133 iterations.

Table A.4.19 Myopathy MUAP - reinnervation of 25 fibres in the right plane of the MU, results after 5295 iterations. 


\section{ABBREVIATIONS AND SYMBOLS}

MU

MUAP

SFEAP

EMG

EAP

ENG
Motor Unit

Motor Unit Action Potential

Single Fibre Extracellular Action Potential

Electromyography

Extracellular Action Potential

ElectroNeuroGraphy 


\section{INTRODUCTION}

There are more than 40 neuromuscular disorders that affect the brain and spinal cord, nerves or muscles. Many of these diseases are hereditary and the life expectancy of many sufferers is considerably reduced. Early detection and diagnosis of these diseases by clinical examination and laboratory tests, is essential for their management as well as their prevention through prenatal diagnosis and genetic counselling. Laboratory investigations include neurophysiologic tests, nerve and muscle biopsies, biochemical analysis and more recently DNA analysis for the localization of genes are currently used. For Duchenne and Becker muscular dystrophy, the dystrophy gene has been identified. These advances have opened new approaches in the management, prevention, and eradication of these diseases. In this study, a new method of describing electromyographic signals into its constituent components is investigated.

Electromyography (EMG) studies the electrical activity of the muscle, and forms a valuable aid in the diagnosis of neuromuscular disorders. EMG findings are used to detect and describe different disease processes affecting the motor unit, the smallest functional unit of the muscle. During slight voluntary contraction individual units are recorded, known as Motor Unit Action Potentials (MUAPS). The motor unit action potential (MUAP) represents the spatial and temporal summation of single fibres extracellular action potentials (SFEAPS) belonging to the same motor unit.

MUAP morphology is affected by structural reorganisation of the motor unit that takes place due to disorders affecting peripheral nerve and muscle. MUAP features extracted in the time domain like duration, amplitude and phases are extensively used by the 
neurophysiologist for the assessment of neuromuscular disorders. In this study, a model based decomposition of MUAPS into their constituent SFEAPS is investigated.

\subsection{The Motor Unit Action Potential}

The fluids inside and outside the cells of the body are electrolytic solutions containing 150 to $160 \mathrm{mEq}$ per litre of positive ions and the same concentration of negative ions.

Generally an excess of anions is accumulated immediately inside the cell membrane along its inner surface and an equal number of kations is accumulated immediately outside the membrane. The result of this is the establishment of a membrane potential between the inside and outside of the cell.

There are two basic mechanisms that develop membrane potentials:

a. Active transport of ions through the membrane, thus creating an imbalance of negative and positive charges.

b. Diffusion of ions through the membrane as a result of ion concentration differences between the two sides of the membrane thus also creates an imbalance of charges.

At resting conditions the membrane potential is developed by the first mechanism. An electrogenic pump, sodium potassium, transports three (3) Sodium $\left(\mathrm{Na}^{+}\right)$ions outwards for every two potassium $\left(\mathrm{K}^{+}\right)$inwards. Eventually there comes a point at which the inward diffusion of $\mathrm{Na}^{+}$(due to the low concentration inwards and negative membrane potential developed) equals the outwards pumping. At this point membrane potential averages $-90 \mathrm{mv}$ and is called Resting Potential. 
When the fibre is stimulated (by electrical stimulation, mechanical compression and application of chemicals to membrane) an action potential is developed due to the increased permeability of the membrane to sodium ions, about 5000 - fold, followed instantaneously by return of the sodium permeability to normal and then potassium permeability increases greatly.

The action potential (Fig. 1.1) moves along the length of the nerve, giving rise to the nerve signal. The action potential occurs in two stages called membrane depolarisation and membrane repolarisation.

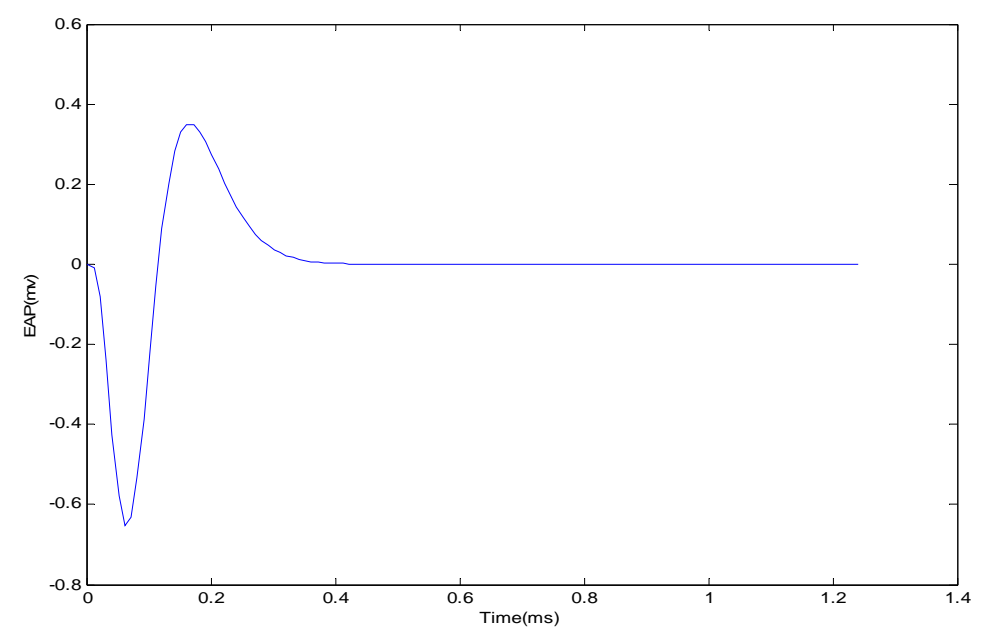

Fig. 1.1 Single Fibre Extracellular Action Potential (EAP).

A motor unit potential is the summation of the spatially and temporally dispersed action potentials of individual muscle fibres belonging to the motor unit. The depolarisation process travels in both directions along the entire extent of the fibre. This transmission is called nerve or muscle impulse.

\subsection{Aim}

The aim of this study was to develop a system that will give to the neurophysiologist a visualization of an "estimated" structural organisation of the motor unit, which includes 
information about number of fibres, fibre distribution and positioning and fibre diameter. This consists of the inverse problem in EMG.

\subsection{Objectives}

The overall objectives of this study were to describe the different methods developed for the simulation of a single fibre action potential, to choose and apply the most appropriate model in order to simulate SFEAPS, simulate MUAPS and decompose them to SFEAPS.

\subsection{Guide to Thesis Contents}

In Chapter 2, human motor unit anatomy is introduced and the basic single fibre action potential (SFEAP) modelling techniques are presented. Also the computer aided electromyography is described and other EMG and ENG Decomposition Techniques are presented. Chapter 3 describes the method followed in this study which consists of the simulation of SFEAP based on a dipole model, the simulation of MUAP (normal and myopathic) as a summation of SFEAPS and finally the decomposition of MUAPS into their constituent SFEAPS. The simulation was developed for two and threedimensional models with a recording radius up to $5 \mathrm{~mm}$ and with a maximum number of fibres up to 50 . In Chapter 4 , the values that were used for the simulation of each model as well as the results obtained from the decomposition are presented analytically. In Chapter 5, the experiments and the results obtained in this study are discussed. In Chapter 6, the conclusions and the future work are given where decomposition of real signals and different algorithms based on evolutionary computation are proposed. 


\section{EXTRACELLULAR POTENTIAL MODELLING AND MUAP SIGNAL}

\section{ANALYSIS}

\subsection{The Motor Unit}

A skeletal muscle is made of numerous fibres (cells) ranging between 10 and 80 microns in diameter. Groups of muscle fibres are supplied by the terminal branches of one nerve fibre or axon whose cell body lies in the anterior horn of the spinal grey matter. This nerve cell body, the long axon, the terminal branches and all the muscle fibres supplied by these branches, together constitute a motor unit (Fig. 2.1). So, the motor unit is the smallest functional unit of muscle, since an impulse descending the nerve axon causes all the muscle fibres in one motor unit to contract almost simultaneously.

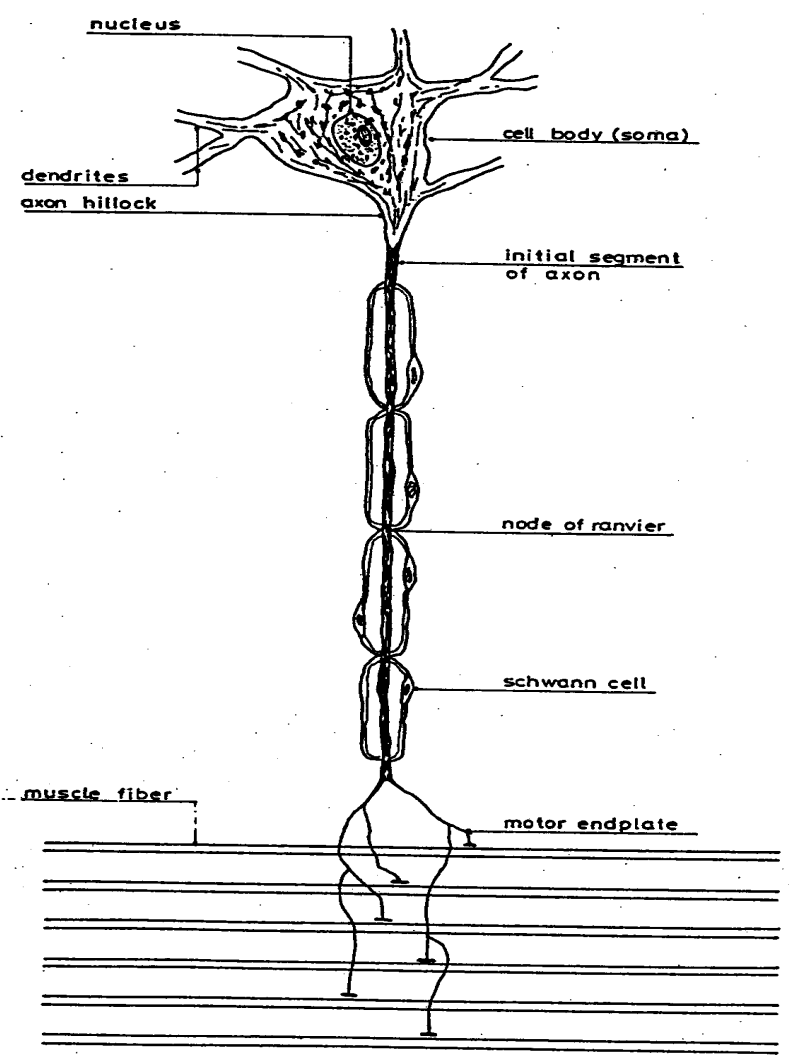

Fig. 2.1 Motor Unit 
The number of muscle fibres that are innervated by one motor neuron i.e. the number in a motor unit varies. In general small muscles that react rapidly and whose control is exact have few muscle fibres in each motor unit like the eyeball and larynx muscles.

On the other hand, large muscles which do not require a very fine degree of control, such as the gastronomies muscle, may have several hundred muscle fibres in a motor unit. On average motor units consist of 150 muscle fibres.

Usually an overlapping exists between fibres of one motor unit and fibres of another motor unit. This overlapping allows the separate motor units to contract in support of each other.

Loss of some of the nerve fibres from a muscle causes the remaining nerve fibres to sprout forth and innervate many of the paralysed muscle fibres. In this case a macro motor unit is developed but it has smaller degree of control, e.g. poliomyelitis causes this phenomenon.

\subsection{Single Fibre Action Potential Modelling}

Six mathematical models in the Single Fibre Action Potential modelling were investigated as follows:

- Core-conduction model (Weerd 1984).

An electric analog simulates the fibre and the medium.

- Volume Conduction as a filter process (Greco, Clark and Harman, 1977). The model is considered as two filters consisting of the membrane and the 
medium filter.

- Dipole Model (Boyd and Lawrence, 1978).

The muscle fibre is considered as a dipole generator consists of a source of current I and a sink of current I.

- Dipole Model (Dimitrova 1974, Dimitrov 1987).

The muscle fibre is considered as a dipole generator as the sum of potentials generated by equivalent dipoles distributed within the fibre length.

- $\quad$ Tripole Model (Rosenfalck, 1969)

The muscle fibre is considered as a tripole generator.

- $\quad$ Line Source Model (Nandedkar, Stalberg, 1983)

The single fibre action potential is viewed as the output of a linear system.

The details and the limitations of these models are described below. The dipole model (Dimitrova 1974, Dimitrov 1987) was selected to be applied as the most appropriate for the MUAP modelling under investigation in this study, as it is widely used, it is more close to real world and it can be applied as a convolution in MATLAB program. Changes in the basic assumptions about the MU architecture affect the results of the simulations. This step consists of the forward problem. The inverse problem has been faced as the decomposition of the MUAP into the constituent SFEAPS. The system was developed using the MATLAB program and consists of two main components, the simulation of MUAPS and the decomposition of the MUAPS into their constituent SFEAPS. 


\subsubsection{Core-Conduction Model (Weerd 1984)}

\subsubsection{Model Details}

Clinical electromyography is based on the measurement of extracellular phenomena i.e. on potential variations. In the core-conduction model it is assumed that a single (thin) fibre is surrounded by an infinitely extended, homogeneous and isotropic medium. However this is not realistic. In practice, the external medium is bound, is isotropic and is inhomogeneous. Potential differences are computed assuming a point-shaped electrode of negligible dimensions constituting the active electrode in the potential field and a second or indifferent electrode situated completely outside this field, or an indifferent electrode situated in the potential field but with large dimensions.

This model is illustrated in Fig. 2.2 in the form of an electric analog where an unmyelinated nerve fibre is assumed where $V i$ is the intracellular potential and $V e$ is the extracellular potential, $R i$ and $R e$ are the resistances per unit of length of intracellular and extracellular membrane and $\mathrm{Zm}$ is the membrane impedance.

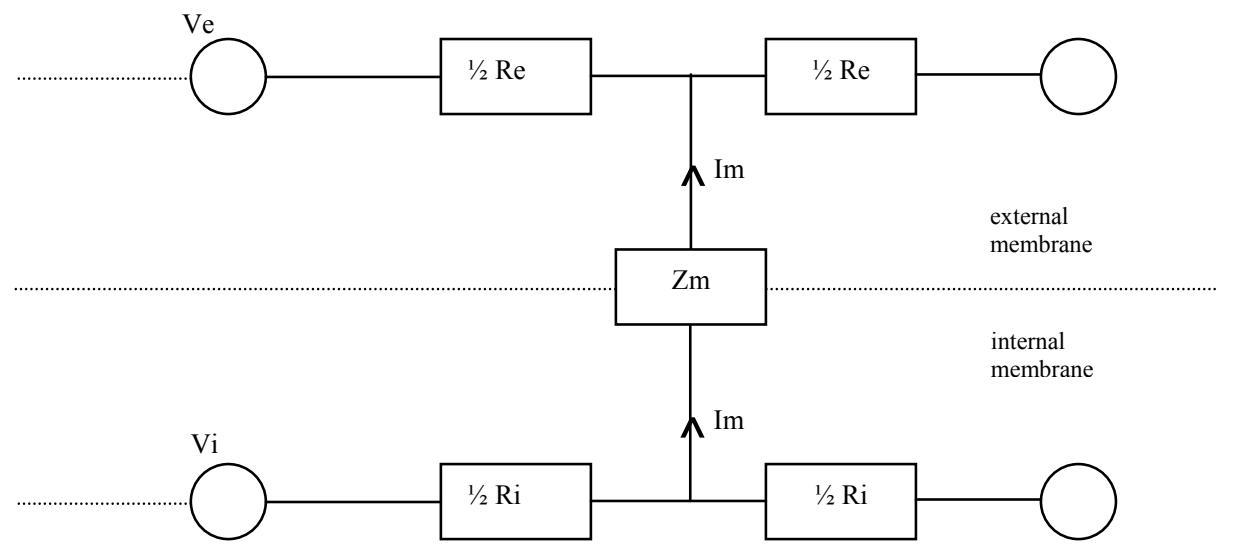

Fig. 2.2 Representation of the core-conduction model as an electric analog. 


\subsubsection{Limitations}

The limitations of the core-conduction model are as follows:

According to this model the extracellular potential measured at the surface of an active fibre is proportional, but opposite to, the intracellular potential. Hence the extracellular action potential should be monophasic as with the intracellular action potential. However this situation is only valid if the fibre is in oil or in air. If the nerve is in situ or immersed in a physiological saline solution, the extracellular currents will spread out in space and the extracellular action potential becomes triphasic.

\subsubsection{Volume Conduction as a Filter process (Greco, Clark and Harman, 1977)}

\subsubsection{Model Details}

Volume conduction has been considered as a filter process with two filters: membrane filter and medium filter. According to this, the extracellular action potential at the outer surface arises by filtering the intracellular action potential. The action potential in the external medium arises by the surface potential. The membrane filter may be regarded as a high pass filter and the medium filter as a low pass filter. The block diagram realization of the filter is given in Fig. 2.3.

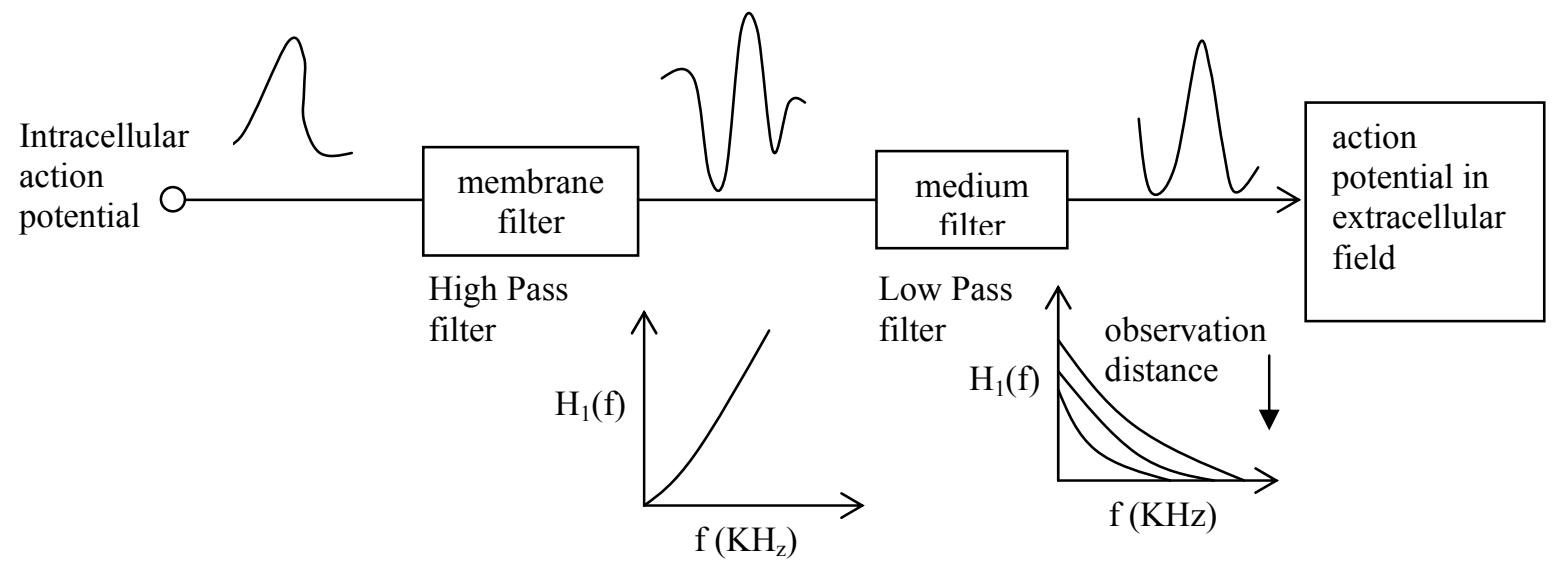

Fig. 2.3 Filter process. 
The mathematical implementation of the filter process is given in Fig. 2.4, where $F_{m}(k), F_{s o}(k), F^{0}(k, r)$ represent the Fourier transforms of trans-membrane, surface and field potential distributions.

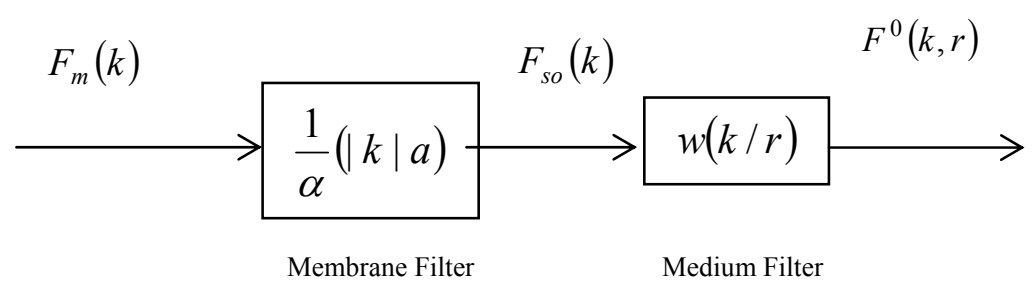

Fig. 2.4 Mathematical implementation of the filter process.

The forward problem is simulated based on the computation of the potential at an arbitrary field point in the extracellular medium.

The data given to the algorithm is:

\section{Transmembrane Action Potential}

2. Electrical and geometrical data, including the fibre radius, the conduction velocity and the conductivities of the intra and extracellular media.

The inverse problem is simulated based on the computation of the transmembrance action potential.

The data given to the algorithm is:

The potential distribution at an arbitrary radial distance $r$ from the fibre with the same electrical and geometrical data as above.

The problem is formulated as a one dimension linear filtering problem and allows the application of well known techniques in linear systems theory and optimal linear filtering (Discrete Fourier Transform DFT) for convolution and least-mean-square (Greco, Clark and Harman, 1977). 
The inverse problem is affected by noise (modelling error and measurement error).

\section{The Model}

A circular cylindrical model of the axon is utilised and simulated action potential data is utilised to produce a "forward" solution at a particular radial distance $r$ from the fibre. The resultant field potential is corrupted with additive Gaussian "measurement" noise and serves as an input to an inverse filter which provides the best estimates in leastsquares sense to the original transmembrane potential data.

The model assumes the intra- and extracellular media to be uniform, homogeneous and purely passive (all sources for the potential being assumed to lie within the fibre membrane), quasi-stationarity (propagation effects are neglected) and axial symmetry in cylindrical coordinates is assumed. $\left(\frac{\vartheta}{\vartheta \phi}=0\right)$

The model is illustrated in Figure 2.5 where $\sigma_{0}$ is the conductivity of the external medium and $\sigma_{\mathrm{i}}$ is the conductivity of the internal medium, $a$ is the fibre radius, $r$ is the radius to the field point and $z$ is the axial dimension.

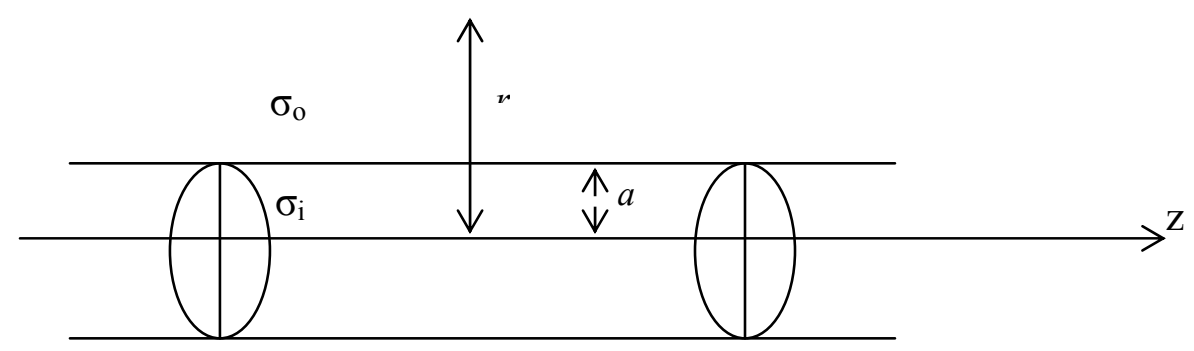

Fig. 2.5 The volume conduction model. 


\section{The forward problem}

Extracellular potential $\Phi^{o}$ at an arbitrary field point from a filter of radius $a$ is given by Clark and Plonse (1968) as

$\Phi^{0}(r, z)=\frac{1}{2 \pi} \int_{-\infty}^{\infty} \frac{F(k) K_{0}(|k| r) e^{-j k z}}{\alpha(|k| a) K_{0}(|k| a)} d k$

where $F_{m}(k)$ is the Fourier transform of the trans-membrane potential distribution $\Phi_{m}(z)$, that is,

$F_{m}(k)=\int_{-\infty}^{\infty} \Phi_{m}(z) e^{j k z} \cdot d z$

and

$\Phi_{m}(z)=\frac{1}{2 \pi} \int_{-\infty}^{\infty} F_{m}(k) \cdot e^{-j k z} \cdot d k$

The term $\alpha(|k| a)$ in equation (2.1) is:

$\alpha(|k| a) \equiv-\left[1+\frac{\sigma_{o}}{\sigma_{\imath}} \frac{\mathrm{K}_{1}(|k| a) \mathrm{I}_{o}(|k| a)}{\mathrm{K}_{0}(|k| a) \mathrm{I}_{1}(|k| a)}\right]$

where $\mathrm{K}_{0}$ is the modified Bessel Function of the second kind (order 0 ) and $I_{l}$ is the modified Bessel Function of the first kind (order 1). The constants $\sigma_{0}$ and $\sigma_{i}$ are the specific conductivities of the extra- and intracellular media, respectively.

In equation 2.1, if $r=r^{*}=$ constant value of radial distance, then it takes the form of a Fourier integral. 
LET $F^{0}\left(k r^{*}\right) \equiv H\left(|k| r^{*}\right) F_{m} \cdot(k)$

where

$$
H\left(|k| r^{*}\right) \equiv \frac{K_{0}\left(|k| r^{*}\right)}{\alpha(|k| a) \cdot K_{0}(|k| a)}
$$

Substituting (2.5) into (2.1) then we obtain the Fourier transform at $r=r^{*}$ in the external medium:

$$
\begin{aligned}
& \Phi^{0}\left(r^{*}, z\right)=\frac{1}{2 \pi} \int_{-\infty}^{\infty} F^{0}\left(k r^{*}\right) e^{-j k z} d k \\
& F^{0}\left(k r^{*}\right)=\int_{-\infty}^{\infty} \Phi^{0}\left(r^{*}, z\right) e^{j k z} d k
\end{aligned}
$$

At the outer membrane surface of the fibre $r^{*}=a$, the potential $\Phi_{s o}(z)$ is given by

$$
\Phi_{s o}(z) \equiv \Phi^{0}(a, z)=\frac{1}{2 \pi} \int_{-\infty}^{\infty} F^{0}(k a) e^{-j k z} d k
$$

The Fourier transform of the outer membrane surface potential is

$$
F_{s o}(k) \equiv F^{0}(k a)
$$

The following Fourier transform pair is established at the membrane surface:

$$
\begin{aligned}
& \Phi_{s o}(z)=\frac{1}{2 \pi} \int_{-\infty}^{\infty} F_{s o}(k) e^{-j k z} d k \\
& F_{s o}(k)=\int_{-\infty}^{\infty} \Phi_{s o}(z) e^{j k z} d k
\end{aligned}
$$


From equations (2.5) and (2.6), at $r^{*}=a$,

$F_{s o}(k)=H(|k| a) F_{m}(k)=\frac{F_{m}(k)}{\alpha(|k| a)}$

So equation (2.5) may also be written as

$$
F^{0}\left(k r^{*}\right)=H\left(|k| r^{*}\right) \alpha(|k| a) F_{s o}(k)
$$

and equation (2.7) may be written as

$$
\Phi^{0}\left(r^{*}, z\right)=\frac{1}{2 \pi} \int_{-\infty}^{\infty} W\left(|k| r^{*}\right) F_{s o}(k) e^{-j k z} d k
$$

where, utilising equation (2.6)

$$
W\left(|k| r^{*}\right) \equiv H\left(|k| r^{*}\right) \alpha(|k| a)=\frac{K_{0}\left(|k| r^{*}\right)}{K_{0}(|k| a)}
$$

From equation (2.14) and the Fourier transform of $\Phi^{0}\left(r^{*}, z\right)$

$$
F^{0}\left(k r^{*}\right)=W\left(|k| r^{*}\right) F_{s o}(k)=\int_{-\infty}^{\infty} \Phi^{0}\left(r^{*}, z\right) e^{j k z} d z
$$

The potential distribution in $z$ at an arbitrary radial distance $r^{*}$ from the fibre may be computed by specification of the Fourier transform of either the transmembrane or outer membrane surface potential distribution:

$$
\Phi^{0}\left(r^{*}, z\right)=\frac{1}{2 \pi} \int_{-\infty}^{\infty} H\left(|k| r^{*}\right) F_{m}(k) e^{-j k z} d k
$$


$\Phi^{0}\left(r^{*}, z\right)=\frac{1}{2 \pi} \int_{-\infty}^{\infty} W\left(|k| r^{*}\right) F_{s o}(k) e^{-j k z} d k$

where from equations (2.13) and (2.16)

$H\left(|k| r^{*}\right)=\frac{W\left(|k| r^{*}\right)}{\alpha(|k| a)}$

and as given in equation $(2.13) \quad F_{s o}(k)=\frac{F_{m}(k)}{\alpha(k \mid a)}$

the Fourier transform of $\Phi^{0}\left(r^{*}, z\right)$ is

$$
F^{0}\left(k r^{*}\right)=\alpha^{-1}(|k| a) W\left(|k| r^{*}\right) F_{m}(k)
$$

From the above, it is concluded that the field potential $\Phi^{0}\left(r^{*}, z\right)$ is a result of two-stage attenuation of the transmembrane potential $\Phi_{m}(z)$.

The first stage is associated with membrane (equation 2.13) where the filter

characteristic is $\alpha^{-1}[(|k| a)]$ and the second stage is characteristic of the external medium (equation 2.17) where the filter characteristic is $W\left(k \mid r^{*}\right)$.

\section{The inverse problem}

The objective is to solve for $F_{m}(k)$ given the filter characteristics and the potential distribution $\Phi^{0}\left(r^{*}, z\right)$.

Equation (2.20) gives: $F_{m}(k)=\alpha(|k| a) W^{-1}\left(|k| r^{*}\right) F^{o}\left(k r^{*}\right)$ in order 
to obtain: $F_{\text {so }}(k)=W^{-1}\left(|k| r^{*}\right) F^{o}\left(k r^{*}\right)$

From the definitions (2.4) and (2.16) is follows that

$\alpha(|k| a) \rightarrow \infty$ as $k \rightarrow 0$

$W^{-1}\left(|k| r^{*}\right) \rightarrow \infty$ as $k \rightarrow \infty$

Therefore, these inverse filter functions are not defined for all $\mathrm{k}$ in the large $-\infty \leq k \leq \infty$. In particular, the $W^{-1}\left(|k| r^{*}\right)$ function is an inverse filter function associated with extracellular medium. Its properties are such that for band-limited signals as $\Phi^{0}\left(r^{*}, z\right)$, the Fourier transform of the surface potential distribution $F_{s o}(k)$ can be obtained via equation (2.22) for a range of $r^{*}$ values of interest.

The membrane inverse filter function $\alpha(|k| a)$ is well behaved except at $k=0$ and this indicates that the direct current resting potential cannot be recovered in the inverse filtering process.

\subsubsection{Limitations}

The model assumes that the intracellular and extracellular media are uniform, homogeneous and purely passive. Also axial symmetry is assumed. However in real world they don't behave this way. In the real world the media is not uniform or homogeneous. 


\subsubsection{Dipole Model (Boyd and Lawrence, 1978)}

\subsubsection{Model Details}

In this model the extracellular action potential of the human muscle fibre, is simulated by a dipole model.

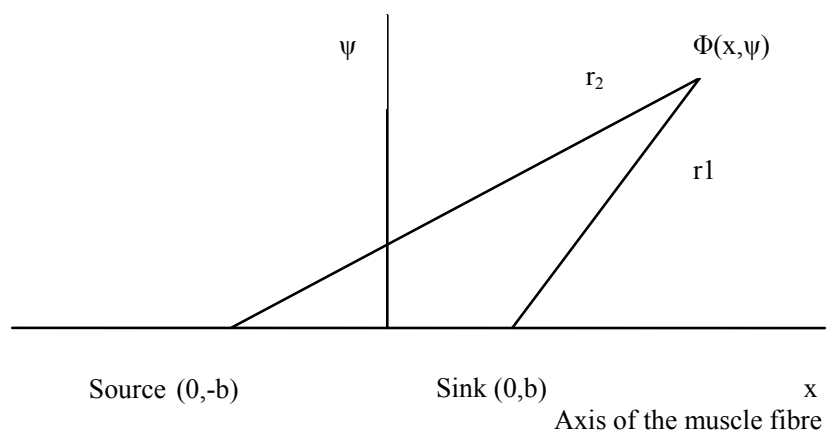

Fig. 2.6 The dipole model.

\section{The Model}

A dipole generator may be considered as consisting of a source of current $I$ and sink of current $I$ at a distance $2 \mathrm{~b}$ as shown in figure 2.6. A current source $I$ produces a spherical potential field $\Phi$ given by:

$\Phi(r)=\frac{I}{4 \pi v r}$

where $r$ is the distance from the source and $v$ is the conductivity of the external medium.

The potential at a point $x, \psi$ is given by

$$
\Phi(\chi, \psi)=\frac{I}{4 \pi v r}\left[\frac{1}{r_{1}}-\frac{1}{r_{2}}\right]
$$

or 
$\Phi(\chi, \psi)=\frac{I}{4 \pi v}\left[\frac{1}{(x+b)^{2}+\psi^{2}}-\frac{1}{(x-b)^{2}+\psi^{2}}\right]$

\subsubsection{Limitations}

The limitations of this model are:

1. The finite diameter of the muscle fibre is not considered.

2. Inhomogenity, anisotropy and finite extent of the volume conductor are not considered either.

\subsubsection{Dipole Model (Dimitrova 1974; Dimitrov 1987)}

\subsubsection{Model Details}

The SFEAP model developed by Dimitrova and Dimitrov (Dimitrova, 1974; Dimitrov, 1987 ) is used. According to the model, the SFEAP is considered to be the sum of external potentials developed by equivalent dipoles distributed within the fibre length and is calculated by the equation:

$$
\Phi\left(x_{0}, y_{0}, d, t\right)=-C_{e} \int_{-L_{2}}^{L_{1}} \frac{\vartheta \phi_{i}}{\vartheta x} \frac{\vartheta\left(\frac{1}{r_{a n}}\right)}{\vartheta x} d x
$$

where
$\phi_{i} \quad$ is the intracellular action potential $=A_{1} x^{A_{2}} e^{A_{3} \chi}$ and $\mathrm{A}_{1}, \mathrm{~A}_{2}, \mathrm{~A}_{3}$ are constants
$x=u t \quad u$ is the propagation velocity which is considered constant
$L_{1}, L_{2} \quad$ are the distances between the endplate and the two edges of the fibre

$C_{e}=\frac{d^{2} K_{a n} V_{m} \sigma_{i}}{16_{\sigma_{a n}}} \quad \sigma_{a n}=\sqrt{\sigma_{x} \sigma_{y}}$ 
$d \quad$ is a fibre diameter

$\sigma_{i} \quad$ is the conductivity inside the fibre

$\sigma_{\mathrm{x}} \quad$ is the conductivity along the $\mathrm{x}$ axis

$\sigma_{y} \quad$ is the conductivity along the y axis

$K_{a n} \quad$ is the medium anisotropicity

$x_{0}, y_{0} \quad$ are the horizontal and vertical distances of the electrode from the endplate

$v_{m} \quad$ is the intracellular action potential amplitude which is considered unity

$r_{a n}=\sqrt{\left(x-x_{0}\right)^{2}+K_{a n}\left(y-y_{0}\right)^{2}}$ is a distance between the electrode $\left(x_{0}, y_{0}\right)$ and fibre

endplate, considering the medium anisotropicity.

Equation (2.26) was implemented using a convolution operation as follows:

$$
\Phi\left(x_{0_{k}}, y_{0_{k}}, d_{k}, t\right)=-C_{e}\left(\frac{\vartheta \phi_{i}}{\vartheta x} * \frac{\vartheta\left(\frac{1}{r_{a n}}\right)}{\vartheta x}\right)
$$

According to the model it was shown that the basic quantitative characteristics of the extracellular potential such as amplitude, temporal, integral and spectral depend on the fibre-electrode distance.

\subsubsection{Limitations}

1. The model assumes that the potential field is generated from a human muscle immersed in an infinite, uniform and isotropic volume conductor (Dimitrova 1974). 
2. The proximity of the place of origin of the excitation to the point of recording causes strong changes in the form of the extracellular potential. Upon recording above the motor endplate area the extracellular potentials are of a two-phase type, and outside the motor endplate area are of three-phase type. The axial distance to which the extracellular potential is of the two-phase type increases with the increase in the radial distance of the point of recording to the axis of the fibre (Dimitrova 1974).

\subsubsection{Tripole Model (Rosenfalck, 1969)}

\subsubsection{Model Details}

The muscle fibre is simulated as a tripole generator as illustrated in figure 2.7 and the mathematical implementation is given in using equation (2.28).

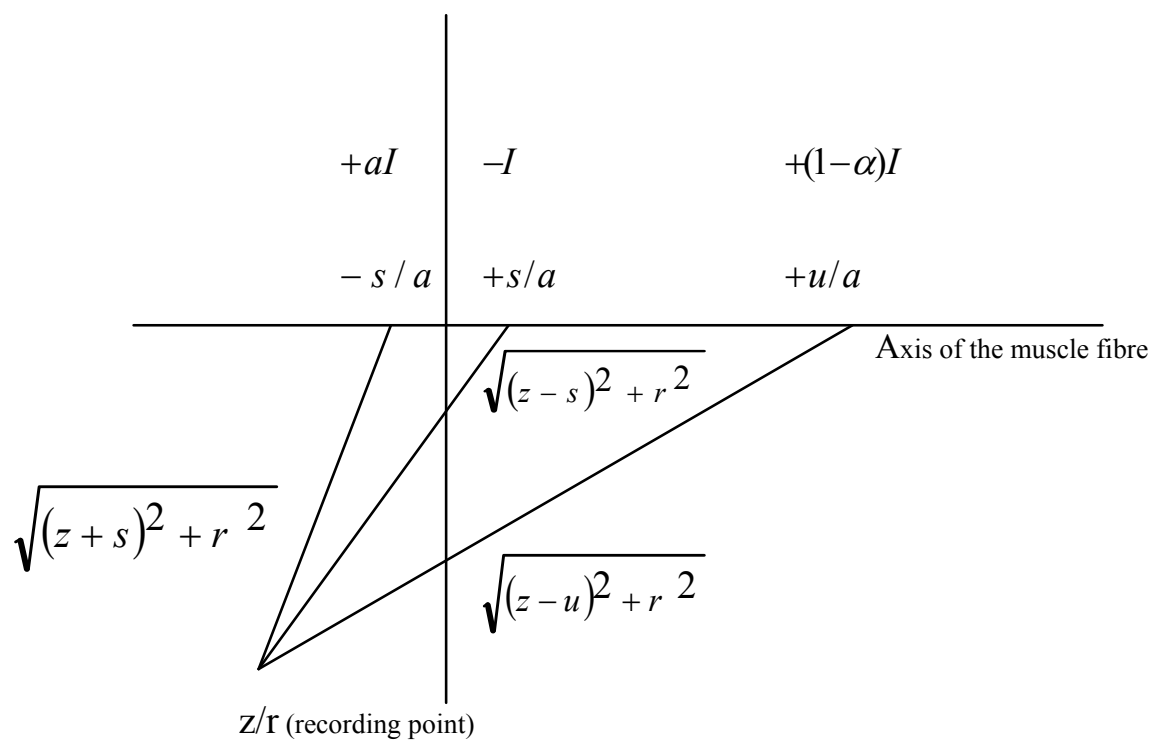

Fig. 2.7 The tripole model.

$\Phi(z, r)=\frac{I}{4 \pi \sigma}\left(\frac{a}{\sqrt{(z+s)^{2}+r^{2}}}-\frac{1}{\sqrt{(z-s)^{2}+r^{2}}}+\frac{1-a}{\sqrt{(z-u)^{2}+r^{2}}}\right)$ 
where

$\sigma: \quad$ conductivity of the volume conductor

$r$ : distance form the recording point perpendicular to the axis through the poles.

$z: \quad$ distance parallel to the three pole axis from the recording point to the centre of the two first poles, which are representing the depolarisation phase.

$s: \quad$ distance from the centre to each of the two first poles.

$u$ : distance to the third pole representing the depolarisation.

I: $\quad$ the current through the second pole.

$\alpha: \quad$ the part distributed to the first pole.

\subsubsection{Limitations}

1. Finite diameter of the muscle fibre was not considered.

2. Inhomogenity, anisotropy were not considered either.

\subsubsection{Line Source Model (Nandedkar and Stalberg 1983)}

\subsubsection{Model Details}

The muscle fibre is assumed to be straight and cylindrical. The extracellular medium is assumed to be infinite with cylindrical anisotropy. The action potential originates at the endplate $(z=0)$ and propagates with a constant velocity towards to tendons so the transmembrane current can also be considered to originate at the endplate and propagate towards the tendons.

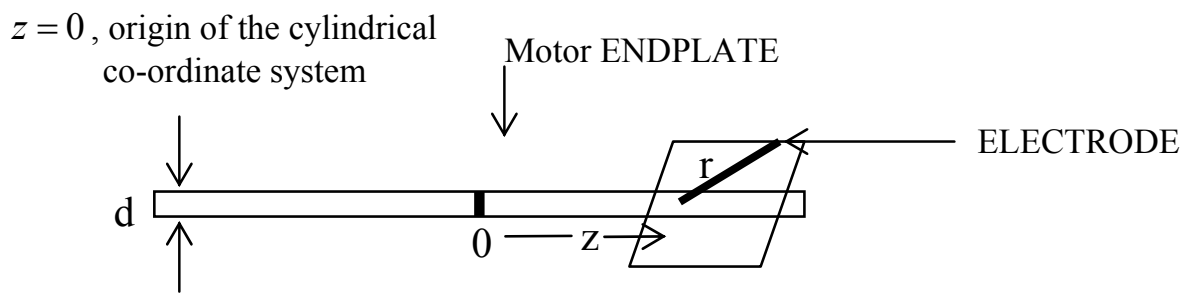

Fig. 2.8 The line source model. 
The current at the endplate is $i_{o}(t)$. The potential produced by a point current source located at the origin of a cylindrical co-ordinate system is:

$\Phi(r, z)=\frac{1}{4 \pi \sigma_{r}}\left(\frac{I}{\sqrt{k r^{2}+z^{2}}}\right)$

where $I=$ strength of current source

$r=$ radial separation

$z=$ axial separation

$\sigma_{r}=$ radial conductivity

$\sigma_{z}=$ axial conductivity

$k=\sigma_{z} / \sigma_{r}$

It is assumed that a unit current source originates at time zero at the endplate and propagates towards the tendons with a constant velocity. $i_{o}(t)$ has been approximated by sources of different amplitudes $\left(a_{1}, a_{2}, \ldots a_{n}\right)$. The first originates at time zero, and each subsequent source at an interval of $\Delta t$.

The first source originates at time 0 , propagates towards the tendons and generates a potential $a_{1} \cdot h(t)$.

The second source originates at the $\Delta t$, produces a potential: $a_{2} \cdot h(t-\Delta t) \therefore \Rightarrow$ Dipole model $n=2$, Tripole model $n=3$

$P(t)=a_{1} \cdot h(t)+a_{2} \cdot h(t-\Delta t)+\ldots+a_{n} h(t-(n-1) \Delta t)$ 
As $\Delta t \rightarrow 0$ and $n \rightarrow \infty$, equation (2.30) represents the convolution of $h(t)$ and $i_{o}(t)$

$$
P(t)=i_{o}(t) * h(t)
$$

As a result the single fibre potential can be viewed as the output of a linear system with the impulse response $h(t)$ and the transmembrane current $i_{o}$ as input.

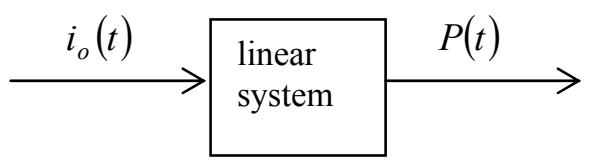

The Fourier transform of equation (2.31) gives:

$$
\begin{aligned}
& P(j w)=I_{o}(j w) \cdot H(j w) \\
& P(t)=F^{-1} P(j w)
\end{aligned}
$$

where

$$
\begin{aligned}
& H(j w)=\int_{-\infty}^{\infty} h(a) \cdot e^{-j w a} d a \\
& I_{o}(j w)=\int_{-\infty}^{\infty} l_{o}(z) \cdot e^{-j w z} d a
\end{aligned}
$$

The time interval used is $40 \mathrm{~ms}$, which satisfies the Nyquist criterion $>\frac{1}{2 f}$

Also Rosenfalck, 1969 described the intracellular action potential as: 
I.A.P $g(z)=96 z^{3} e^{-z}-90$

So the transmembrane current density is proportional to

$g^{\prime \prime}(z)=96 z\left(6-6 z+z^{2}\right) e^{-z}$

The transmembrane current was computed as:

$\operatorname{Im}(z)=\frac{\sigma_{i} d g^{\prime \prime}(z)}{4}$

where $d$ diameter of the fibre

$\sigma_{\imath}=$ intracellular conductivity

Because Rosenfalck found at his experimental data the max value of $g^{\prime \prime}(z)$ to be 400 $\mathrm{mvmm}^{-2}$ and the rise time and duration of the simulated intracellular potential and its second derivative twice of the experimentally recorded, Nandedkar and Stalberg modified Rosenfalck's I.A.P as

$e(z)=g(2 z)$

$e^{\prime \prime}(z)=4 g^{\prime \prime}(2 z)$

and now matches Rosenfalck's experimental data.

The transmembrane current on the surface is lumped at the centre of the fibre and is expressed as:

$I_{m}(z)=\frac{\sigma_{l} \pi d^{2}}{4} e^{\prime \prime}(z)$

Equation (2.39) describes the transmembrane current distribution along the length of the fibre at one instance. 
This is needed to compute the variation of current at the endplate in time.

$z=U \cdot t$

so $l_{o}(t)=I_{m} .(U t)$,

where $U=$ conduction velocity

Output: Function $h(t)$-The potential generated by a unit current source when it propagates from the endplates towards the tendons.

Input: 1. length of fibre $(200 \mathrm{~mm}$ in all simulations $)$

2. fibre diameter

3. conduction velocity

4. distance between the endplate and tendons

5. axial position of the electrode

6. radial position of the electrode

(The same values Rosenfalck 1980)

7. $\sigma_{r}=0.063 \mathrm{sm}^{-1}, \sigma_{z}=0,33 \mathrm{sm}^{-1}, \sigma_{t}=1.01 \mathrm{sm}^{-1}$

$$
\mathrm{K}=\frac{\sigma_{z}}{\sigma_{r}}=5
$$

Note: The potential is independent of the $\Theta$ co-ordinate owing to the cylindrical symmetry.

The source is moved in either direction from the endplate in time intervals of $40 \mu \mathrm{s}$. The potential is computed using the following equation:

$$
\left(\Phi(r, z)=\frac{I}{4 \pi \sigma_{r}}\left(\frac{I}{k r^{2}+z^{2}}\right)\right)
$$


and a 1024 point second of sampled $h(t)$ is constructed (Total duration: 40-96ms).

With conduction velocity $2.2 \mathrm{~ms}^{-1}$ the current source will move about $90 \mathrm{~mm}\left(\approx 2.2 \mathrm{~ms}^{-1} \mathrm{x}\right.$ $40 \mathrm{~ms}$ ) which is roughly equal to the distance between the endplate and the tendons.

\subsubsection{Limitations}

1. A correction factor for amplitude is needed when the source and the electrode are in the same z-plane, as well as when the electrode-fibre centre distance is 1.1 radii.

2. Underestimation of the peak amplitude for large muscle fibre diameters. 


\subsection{Computer Aided Electromyography}

Structural reorganisation of the motor unit, the smallest functional unit of muscle, takes place because of disorders affecting peripheral nerve and muscle. Motor unit morphology can be studied by recording its electrical activity, the procedure known as EMG. In clinical EMG, MUAPS are recorded using a needle electrode at slight voluntary contraction. The MUAP reflects the electrical activity of a single anatomical motor unit. It represents the compound action potential of those muscle fibres within the recording range of the electrode. Features of MUAP's extracted in the time domain such as duration, amplitude, and phases proved to be very valuable in differentiating between muscle and nerve diseases with the duration measure being the key parameter used in clinical practice. However, the measurement of the duration parameter is a difficult task depending on the neurophysiologist and/or the computer-aided method used. The definition of widely accepted criteria that will allow the computer-aided measurement of this parameter, are still lacking. On the other hand, frequency domain features of MUAP's like the mean, or median frequency, bandwidth, and quality factor provide additional information in the assessment of neuromuscular disorders and it has recently been shown that the discriminative power of the MUAP mean or median frequency is comparable to the duration measure or the spike duration measure (Pattichis and Elias, 1999). Furthermore, earlier EMG frequency analysis studies showed a displacement towards lower frequencies for neurogenic lesions, with the opposite being true for myogenic lesions. These findings were also confirmed by Larsson (Larsson, 1975) who devised a method for the automated measurement of the EMG power content of four octave band filters with center frequencies at 50, 200, 800 and $1600 \mathrm{~Hz}$. Even though frequency-domain techniques describe the frequencies present in the signal, information about their occurrence in time is missing. The analysis 
of MUAP signal characteristics in both the time and frequency domains using the wavelet transform was also investigated (Pattichis and Pattichis, 1999). They investigated the following four different wavelets: Daubechies with four (DAU4) and 20 (DAU20) coefficients, Chui $(\mathrm{CH})$ and Battle-Lemarie (BL). It was shown that significant information about MUAP morphology could be extracted. Computer-aided EMG provides indispensable tools in the hands of the neurophysiologist in facilitating quantitative analysis and decision making in clinical neurophysiology, rehabilitation, sports medicine, and studies of human physiology. These tools form the basis of a new era in the practice of neurophysiology facilitating:

(i) Standardisation. Diagnoses obtained with similar criteria in different laboratories can be verified.

(ii) Sensitivity. Neurophysiological findings in a particular subject under investigation may be compared with a database of normal values to determine whether abnormality exists or not.

(iii) Specificity. Findings may be compared with databases derived from patients with known diseases, to evaluate whether they fit a specific diagnosis.

(iv) Equivalence. Results from serial examinations on the same patient may be compared to decide whether there is evidence of disease progression or of response to treatment. Also, findings obtained from different quantitative methods may be contracted to determine which are most sensitive and specific.

In recent years, different methodologies have been developed in computer-aided EMG and ENG analysis ranging from simple quantitative measures of the recorded potentials as described above, to more complex knowledge-based and neural network systems that enable the automated assessment of neuromuscular disorders (Pattichis et. al. 1995, Pattichis et. al.1996, Christodoulou et. al. 1998, Pattichis et. al. 1999). However, the 
need still exists for the further advancement and standardization of these methodologies, especially nowadays with the emerging health telematics technologies, which will enable their wider application in the neurophysiological laboratory.

The main objective of this study was to develop a system that will give to the neurophysiologist a visualization of an "estimated" structural organisation of the motor unit, which includes information about number of fibres, fibre distribution and positioning and fibre diameter. This consists of the inverse problem in EMG.

\subsection{Other EMG and ENG Decomposition Techniques}

To the best of the Author's knowledge, no similar work to this study has been carried out for decomposing simulated MUAPS into their constituent SFEAPS. A similar work has been carried out for decomposing surface EMG into SFEAP based on neural networks (Graupe et. al, 1989). In addition, two studies investigated successfully the decomposition of needle EMG into its constituent MUAPS (Le Fever and De Luca, 1982, McGill et. al. 1985). Moreover, a decomposition of the Compound Action Potential in EMG has been proposed. These decomposition techniques are briefly described below:

\section{A. Decomposition of surface EMG into SFEAPS}

- Decomposition of surface EMG signals into SFEAPS by means of neural networks (Graupe et. al, 1989)

In this study a neural network model has been developed to decompose surface EMG signals into a multitude of below-skin single fibre external action potentials and also a localization of fibres by using subsequent conventional correlation computations. This approach utilizes a Hopfield neural network and it is based on the work by Tank and Hopfield on the decomposition of Gaussian 
sum probability distribution functions (p.d.f.'s). This utilization is facilitated by the nature of SFEAPS that they can be considered to be the sum of three base functions having the form of a Gaussian p.d.f function.

\section{B. Decomposition of needle EMG into its constituent MUAPS}

- Procedure for decomposing the EMG signal into its constituent Action Potentials (LeFever and De Luca Part I and II, 1982)

A technique has been developed which enables the decomposition of a myoelectric signal into its constituent MUAPS. It consists of a recording procedure, a data compression algorithm, a digital filtering algorithm and a hybrid visual decomposition interface. The decomposing algorithm uses a continuously updated template matching routine and firing statistics of the Motor Units to identify MUAPS in the signal recorded.

- Automatic decomposition of the clinical EMG (McGill et. al, 1985)

An automatic signal-processing method has been described for extracting MUAPS from the electromyography interference pattern. The method employs digital filtering to select the spike components of the MUAPS from the background activity, identifies the spikes by template matching, and averages the MUAP waveforms from the raw signal using the identified spikes as triggers.

C. Decomposition of the Compound Action Potential (CAP) in ENG

- The inverse Problem in Electroneurography: conceptual basis and mathematical Formulation (Schoonhoven et. al, 1988) 
A mathematical technique for the analysis of the compound action potential (CAP) of a peripheral nerve has been described which deals with the estimation of the number of active fibres contributing to the CAP and the distribution of their conduction velocities. The CAP has been described as a filtered Poisson process, with the (time-varying) filter representing the SFEAP waveshapes. The procedure consists of two parts. The first part is related to the fast conducting fibres contributing to the CAP and uses least-squares optimisation techniques for the reconstruction to the CAP waveshape. The second part is related to the small late components of the CAP due to the slowly conducting fibres. The model explicitly uses the Poisson process formalism and is based on reconstructing the energy or variance in the CAP.

Differences with my work

This study is different from the studies described in Sections A, B and C. In this study MUAPS were decomposed into their constituent SFEAPS and gives the neurophysiologist a visualisation of an "estimated" structural organisation of the motor unit. 


\section{METHODOLOGY}

\subsection{Simulation of SFEAP}

The model developed by Dimitrova and Dimitrov (Dimitrova 1974; Dimitrov 1987) was used in order to generate SFEAPS where the muscle is considered as a dipole. The model is described in Section 2.2.4. According to the model, the SFEAP is calculated by the equation:

$$
\Phi\left(x_{0}, y_{0}, d, t\right)=-C_{e} \int_{-L_{2}}^{L_{1}} \frac{\vartheta \phi_{i}}{\vartheta x} \frac{\vartheta\left(\frac{1}{r_{a n}}\right)}{\vartheta x} d x
$$

The simulation programs have been developed using MATLAB program, function tot_out3.m (Appendix 1) for a two-dimensional model and function extra.m (Appendix 2) for a three-dimensional model. The equation (3.1) has been implemented as a convolution within a specific time interval as follows:

$$
\Phi\left(x_{0_{k}}, y_{0_{k}}, d_{k}, t\right)=-C_{e}\left(\frac{\vartheta \phi_{i}}{\vartheta x} * \frac{\vartheta\left(\frac{1}{r_{a n}}\right)}{\vartheta x}\right)
$$

\subsection{Simulation of MUAP}

The structural organisation of normal motor units in human biceps brachii muscle was used as the basis of the simulations. A similar structure was also used by Nandedkar and co-workers (Nandedkar et. al. 1988). Motor units were simulated by randomly distributing 50 muscle fibres within a circular territory of $5.64 \mathrm{~mm}$ diameter. The mean and standard deviation of the diameter of muscle fibres in the motor unit were $50 \mu \mathrm{m}$ and $28 \mu \mathrm{m}$, respectively (Dubovitz and Brooke 1973). The endplates were assumed to 
be distributed within a $5 \mathrm{~mm}$ wide zone midway along the length of the muscle fibres (Aquilonius et. al. 1984). It was assumed that the recording electrode was positioned at the centre of the motor unit circular territory, at the centre of the endplate zone. The MUAP was computed by summing the SFEAPs within a predetermined semicircular recording-testing area facing the electrode as follows:

$$
\Phi\left(t_{i}\right)=\sum_{k=1}^{M} \Phi\left(x_{k}, y_{k}, d_{k}, t_{i k}\right) \quad i=1, \ldots, N
$$

where $\mathrm{M}$ is the number of fibres positioned within the testing area

$\mathrm{N}$ is the maximum number of sample points.

In the first case, a MUAP has been simulated as a sum of SFEAPS generated for 1, 4, 5 and 10 fibres enclosed within a semicircular area of radii $0.6,0.9,1.0$ and $1.2 \mathrm{~mm}$. The function used is invmuap.m and it is attached in Appendix 1.

The experiments start with 1 fibre in order to determine the behaviour of a single fibre before extending the model.

In the second case, a MUAP has been simulated as a sum of SFEAPS generated for 50 fibres enclosed within a semicircular area of radii $5 \mathrm{~mm}$. In this case, a third dimension has been added and also the model has been applied for myopathic cases. The functions used (normal2.m, diam2.m, rein1.m, rein2.m) are attached in Appendix 2.

\subsection{Decomposition}

The decomposition of simulated MUAPS into their constituent SFEAPS was addressed as an unconstrained optimization problem. The non-linear least squares optimization 
procedure based on the Levenberg-Marquardt algorithm (More, 1977) was used to obtain a solution to the MUAP decomposition problem i.e. fibre distribution and positioning and fibre diameter. The problem to be solved was formulated as follows:

$$
\begin{aligned}
& \text { minimize } \\
& \qquad p \varepsilon R^{n} \quad\left\{F(p)=\sum_{i=1}^{N}\left(R\left(p, t_{i}\right)-\Phi\left(t_{i}\right)\right)^{2}\right\}
\end{aligned}
$$

where $\mathrm{p}=\left[\mathrm{x}_{01}, \mathrm{y}_{01}, \mathrm{~d}_{1}, \ldots . . \mathrm{x}_{0 \mathrm{M}}, \mathrm{y}_{0 \mathrm{M}}, \mathrm{d}_{\mathrm{M}}\right]$ is the $\mathrm{n}$-dimensional unknown vector, $\mathrm{R}$ is a reconstructed MUAP defined as follows:

$$
R(p, t)=-C_{e} \int_{-L_{2}}^{L_{1}} \frac{\vartheta \phi_{i}}{\vartheta x} \frac{\vartheta\left(\frac{1}{r_{a n}}\right)}{\vartheta x} d x
$$

The equation (3.4) also gives the error function.

The n-dimensional vector has dimensions $1 \mathrm{M}$, where 1 is three representing variables $\mathrm{x}_{0 \mathrm{k}}, \mathrm{y}_{0 \mathrm{k}}, \mathrm{d}_{\mathrm{k}}$ of the SFEAP.

The Levenberg-Marquardt method was used to optimize $F(p)$ as implemented in the MATLAB optimization toolbox. Levenberg-Marquardt is a popular alternative to the Gauss-Newton method of finding the minimum of a function $\mathrm{F}(\mathrm{x})$ that is a sum of squares of non-linear functions $\mathrm{f}_{\mathrm{i}}(\mathrm{x})$,

$$
F(x)=\frac{1}{2} \sum_{i=1}^{m}\left[f_{i}(x)\right]^{2}
$$

Let the Jacobian of $f_{i}(x)$ be denoted as $J_{i}(x)$, then the Levenberg-Marquardt method searches in the direction given by the solution $p$ to the equations $\left(j_{k}^{T}+\lambda_{k} I\right) p_{k}=-J_{k}^{T} f_{k}$, where $\lambda_{\kappa}$ are non negative scalars and $\mathrm{I}$ is the identity matrix. The method has the nice property that, for some scalar $\nabla$ related to $\lambda_{\kappa}$, the vector $p_{k}$ is 
the solution of the constrained subproblem of minimizing $\left\|J_{k} p+f_{k}\right\|_{2}^{2} / 2$ subject to $\|p\| 2 \leq \nabla$ (Gill et. al. 1981, p.136).

The main contributions of this study are:

a. The extension of the two-dimensional model developed by Dimitrova and Dimitrov to a three-dimensional model and;

b. The development of a new method to aid the neurophysiologist in gaining a better assessment of the muscle structural organisation. 


\subsection{Description of the Experiments}

All programs developed using the MATLAB program were based on the dipole model (Dimitrova 1974; Dimitrov 1987), which is described in Section 2.2.4. The steps followed in each model are described, below.

\subsubsection{Simulation and decomposition of a two-dimensional model}

The simulation and decomposition of the two-dimensional model can be divided into five steps as described below.

Step 1. Generate the random numbers to create the components of each fibre using the MATLAB function $\operatorname{rand}(3, n)$ where $n$ is the number of fibres. Each fibre is represented by a column of $x$-horizontal distance, $y$-vertical distance and $d$-diameter. The same generator was used to produce values for $1,4,5$ and 10 fibres in a test radius of $0.6 \mathrm{~mm}$, $0.9 \mathrm{~mm}, 1 \mathrm{~mm}$ and $1.2 \mathrm{~mm}$ respectively. The generator was used to produce simulated and initial values.

Step 2. Apply equation (3.2) $\Phi\left(x_{0_{k}}, y_{0_{k}}, d_{k}, t\right)=-C_{e}\left(\frac{\vartheta \phi_{i}}{\vartheta x} * \frac{\vartheta\left(\frac{1}{r_{a n}}\right)}{\vartheta x}\right)$ using the MATLAB convolution function $\operatorname{conv}($ ).

$\phi_{i} \quad$ is the intracellular action potential $=A_{1} x^{A_{2}} e^{A_{3} X}$ and $\mathrm{A}_{1}=72234, \mathrm{~A}_{2}=5$, $\mathrm{A}_{3=}-11$ are constants.

$x=u t \quad u=4 \mathrm{~m} / \mathrm{s}$ is the propagation velocity which is considered constant.

$L_{1}, L_{2} \quad$ are the distances between the endplate and the two edges of the fibre

$C_{e}=\frac{d^{2} K_{a n} V_{m} \sigma_{i}}{16 \sigma_{a n}}$ where $\sigma_{a n}=\sqrt{\sigma_{x} \sigma_{y}}$ 
d

$\sigma_{\mathrm{i}}=1.01 \quad$ is the conductivity inside the fibre and

$\sigma_{\mathrm{x}}=0.33 \quad$ is the conductivity along the $\mathrm{x}$ axis

$\sigma_{\mathrm{y}}=0.63 \quad$ is the conductivity along the $\mathrm{y}$ axis

$\mathrm{K}_{\mathrm{an}}=5 \quad$ is the medium anisotropicity

$\mathrm{x}_{0}, \mathrm{y}_{0}$

$\mathrm{V}_{\mathrm{m}}=1$ are the horizontal and vertical distances of the electrode from the endplate and considered 0 .

$r_{a n}=\sqrt{\left(x-x_{0}\right)^{2}+K_{a n}\left(y-y_{0}\right)^{2}}$ is a distance between the electrode $\left(\mathrm{x}_{0}, \mathrm{y}_{0}\right)$ and fibre

endplate, considering the medium anisotropicity.

Step 3. Develop the model using equation (3.2) to simulate a SFEAP. The function tot_out3.m (Appendix 1) was used.

Step 4. Sum the SFEAPS using equation (3.3) to produce the MUAP in all cases.

Step 5. Develop the program invmuap.m (Appendix 1) to decompose the MUAP into its constituent SFEAPS using equation (3.4). The program applied the non-linear leastsquares optimization procedure based on Levenberg-Marquardt algorithm, by using the matlab function leastsq(). In each case the simulated and the forecasted signals are compared. The forecasted signals are developed by applying the above model using initial values. 


\subsubsection{Simulation and decomposition of a three-dimensional Model}

The simulation and decomposition of the three-dimensional model can be divided into five steps as follows:

Step 1. Generate random numbers to create the components of each fibre by using the MATLAB function rand(5,50) which generates 5 columns for each fibre with the total number of fibres equal to 50 in a test radius of $5 \mathrm{~mm}$. Each fibre is represented by a column of $x$-horizontal distance, $y$-vertical distance, $z$-z distance, $d$-diameter and $t$ delay. The generator was used to produce simulated and initial values.

Step 2. Apply equation (3.2) with the difference that a third dimension $z$ was included. Therefore the distance between the electrode $\left(\mathrm{x}_{0}, \mathrm{y}_{0}, \mathrm{z}_{0}\right)$ and the fibre endplate, considering the third dimension $z$ is $\quad r_{a n}=\sqrt{\left(x-x_{0}\right)^{2}+\mathrm{K}_{a n}\left(y-y_{0}\right)^{2}+\mathrm{K}_{\mathrm{an}}\left(\mathrm{z}-\mathrm{z}_{0}\right)^{2}}$

Step 3. Develop the model using equation (3.2) to simulate a SFEAP. The function extra.m (Appendix 2) was used.

Step 4. Sum the SFEAPS using equation (3.3) to produce the MUAP using the function simu0208.m (Appendix 2).

Step 5. Develop the program normal2.m (Appendix 2) to decompose the MUAP into it's constituent SFEAPS using equation (3.4). The program applied the non-linear leastsquares optimisation procedure based on Levenberg-Marquardt algorithm, by using the MATLAB function leastsq( ). The program was executed for 251 iterations, 1011 iterations, 5065 iterations and the simulated and forecasted signals are compared. The forecasted signals are developed by applying the above model using initial values. 


\subsubsection{Simulation and decomposition of a three-dimensional model in the case of a} myopathy

Three different cases of myopathy were examined:

- Variability in diameter, where the mean diameter of the fibres changes.

- Loss of half of the fibres

- Reinnervation of fibres. Twenty five fibres were added:
a. In the whole MU territory and
b. In the right plane of the MU territory.

The same steps were used as in normal cases (3.4.2 above). Functions used are diam2.m, halfloss. $m$, rein $1 . m$, rein22.m respectively and they are given in Appendix 2.

Extending the model to three dimensions and adding more fibres increases the computational complexity and performance of the algorithm. 


\section{RESULTS}

\subsection{MUAP Simulation and Decomposition Using a Two-Dimensional Model}

MUAP simulations with $1,4,5$ and 10 fibres using a two-dimensional model were carried out. For each experiment, the simulated values, initial values and final results (forecasted values) are given for the $x$-horizontal distance, $y$-vertical distance and $d$ diameter. Plots of the position of the coordinates of the simulated and final values, as well as the simulated and final waveforms are given.

\subsubsection{MUAP simulation for 1 fibre}

Table 4.1 gives the simulated, initial and final results for the $x$ and $y$ coordinates and diameter, $d$. It is clearly shown that the final results are very close to the simulated values in 17 iterations with an infinitely small error. Figure 4.1 illustrates the closeness of the coordinates between the simulated and final values as well as the overlap between the simulated and final waveforms.

\begin{tabular}{ll}
\hline Simulated Values $(\mathrm{r} 1) \mathrm{mm}$ \\
\hline $\mathrm{x}$ & 0.3750 \\
$\mathrm{y}$ & 0.3946 \\
$\mathrm{~d}$ & 0.0328 \\
\hline Initial Values $(\mathrm{z} 1) \mathrm{mm}$ \\
\hline $\mathrm{x}$ & 0.4000 \\
$\mathrm{y}$ & 0.4000 \\
$\mathrm{~d}$ & 0.0400 \\
\hline Results $(\mathrm{o} 1) \mathrm{mm}$ \\
\hline $\mathrm{x}$ & 0.3749 \\
$\mathrm{y}$ & 0.3945 \\
$\mathrm{~d}$ & 0.0328 \\
\hline Error=4.7475e-011
\end{tabular}

Table 4.1 MUAP simulation and decomposition for 1 fibre. 

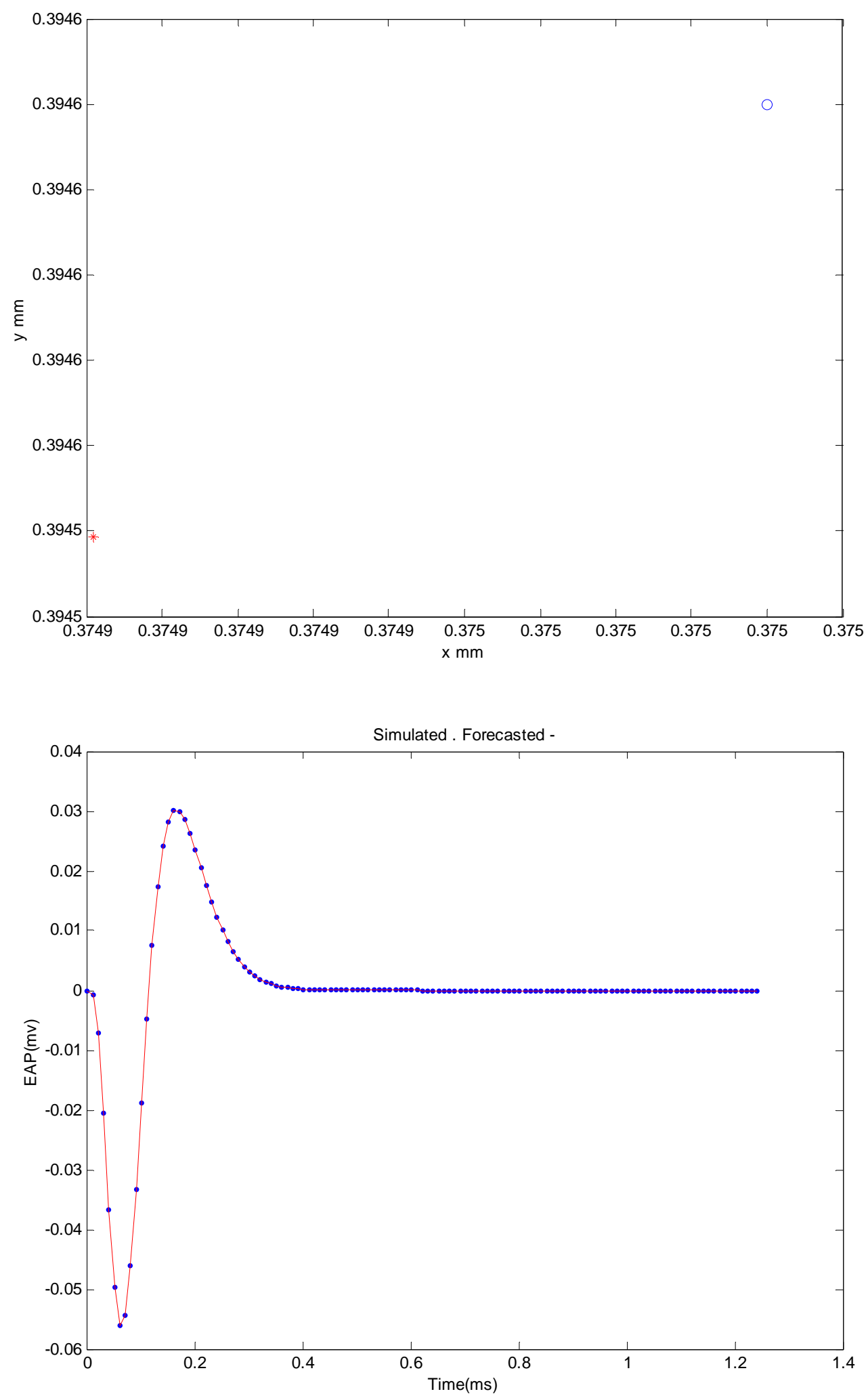

Fig. 4.1 Simulation and decomposition of 1 fibre for a test radius of $0.6 \mathrm{~mm}$. (simulated blue '.', forecasted red '-')

The top graph shows the positioning of the fibres.

(simulated blue 'o', forecasted red '+') 


\subsubsection{MUAP simulation for 4 fibres}

Table 4.2 gives the simulated, initial and final results for the $x$ and $y$ coordinates and diameter, $d$. It is clearly shown that the final results are very close to the simulated values in 58 iterations with an infinitely small error. Figure 4.2 illustrates the closeness of the coordinates between the simulated and final values as well as the overlap between the simulated and final waveforms.

\begin{tabular}{|c|c|c|c|c|}
\hline \multicolumn{5}{|c|}{ Simulated values (r4) mm } \\
\hline \multirow[t]{4}{*}{ Fibre no: } & 1 & 2 & 3 & 4 \\
\hline & 0.0751 & 0.3750 & 0.3180 & -0.3367 \\
\hline & 0.5817 & 0.3946 & 0.6158 & 0.6348 \\
\hline & 0.0679 & 0.0328 & 0.0983 & 0.0478 \\
\hline \multicolumn{5}{|c|}{ Initial Values (z4) mm } \\
\hline Fibre no: & 1 & 2 & 3 & 4 \\
\hline \multirow{2}{*}{$\begin{array}{l}x \\
y\end{array}$} & 0.0800 & 0.4000 & 0.3000 & -0.3300 \\
\hline & 0.6000 & 0.4000 & 0.6200 & 0.6500 \\
\hline $\mathrm{d}$ & 0.0700 & 0.0300 & 0.1000 & 0.0500 \\
\hline \multicolumn{5}{|c|}{ Results (o4) mm } \\
\hline Fibre no: & 1 & 2 & 3 & 4 \\
\hline \multirow[t]{3}{*}{7} & 0.0804 & 0.4001 & 0.3005 & -0.3299 \\
\hline & 0.5999 & 0.3995 & 0.6191 & 0.6502 \\
\hline & 0.0676 & 0.0333 & 0.0992 & 0.0460 \\
\hline \multicolumn{5}{|c|}{ Error $=1.9997 \mathrm{e}-013$} \\
\hline
\end{tabular}

Table 4.2 MUAP simulation and decomposition for 4 fibres. 

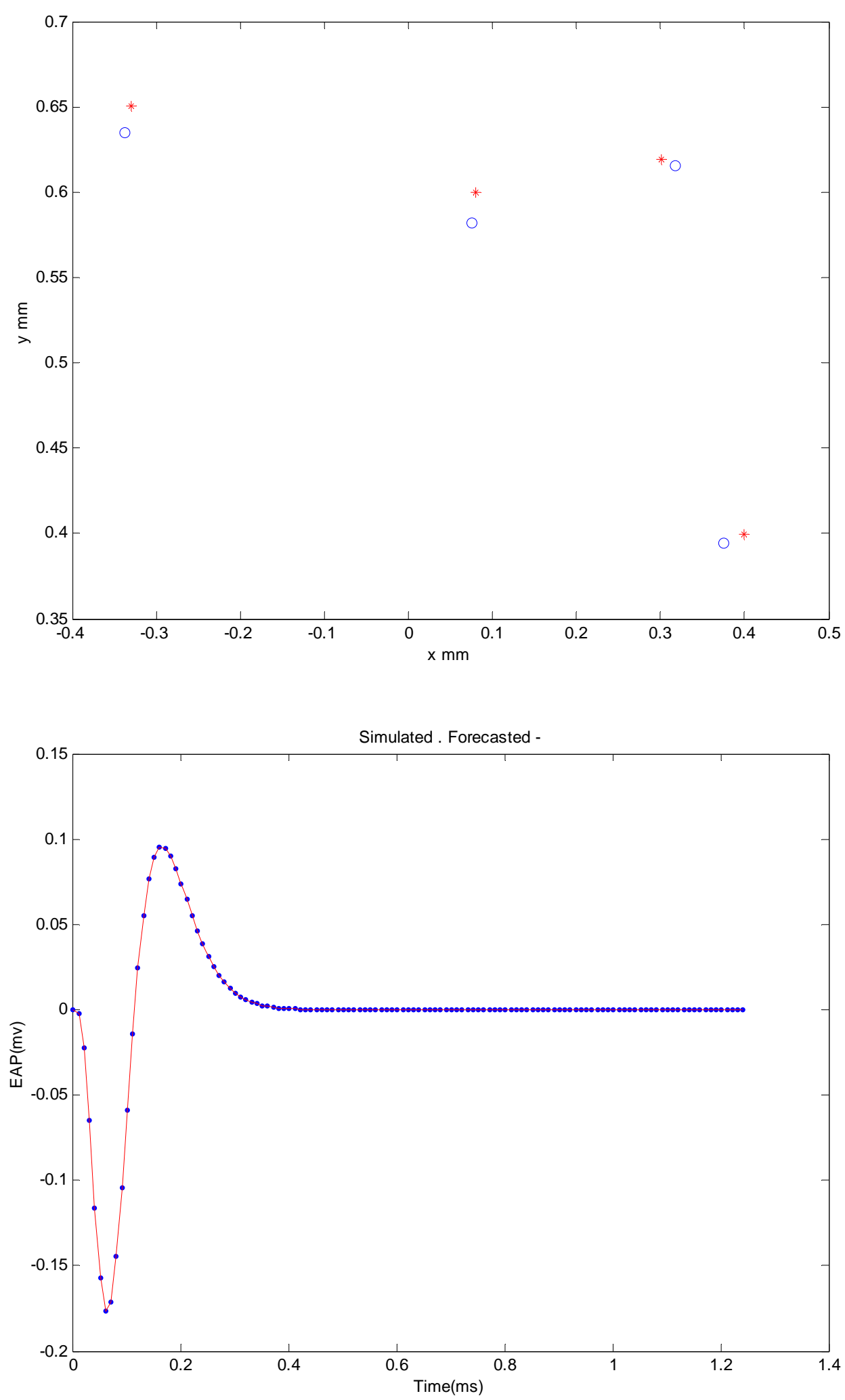

Fig. 4.2 Simulation and decomposition of 4 fibres for a test radius of $0.9 \mathrm{~mm}$. (simulated blue '.' forecasted red '-')

The top graph shows the positioning of the fibres.

(simulated blue 'o', forecasted red '+') 


\subsubsection{MUAP simulation for 5 fibres}

Table 4.3 gives the simulated, initial and final results for the $x$ and $y$ coordinates and diameter, $d$. It is clearly shown that the final results are very close to the simulated values in 53 iterations with an infinitely small error. Figure 4.3 illustrates the closeness of the coordinates between the simulated and final values as well as the overlap between the simulated and final waveforms.

\begin{tabular}{|c|c|c|c|c|c|}
\hline \multicolumn{6}{|c|}{ Simulated values (r5) $\mathrm{mm}$} \\
\hline Fibre no: & 1 & 2 & 3 & 4 & 5 \\
\hline $\mathrm{x}$ & 0.0751 & -0.6965 & 0.3750 & 0.3180 & -0.3367 \\
\hline $\mathrm{y}$ & 0.5817 & 0.4142 & 0.3946 & 0.6158 & 0.6348 \\
\hline $\mathrm{d}$ & 0.0679 & 0.0935 & 0.0328 & 0.0983 & 0.0478 \\
\hline \multicolumn{6}{|c|}{ Initial Values (z5) mm } \\
\hline Fibre no: & 1 & 2 & 3 & 4 & 5 \\
\hline $\mathrm{x}$ & 0.0750 & -0.6960 & 0.3700 & 0.3200 & -0.3370 \\
\hline $\mathrm{y}$ & 0.5800 & 0.4140 & 0.3950 & 0.6160 & 0.6350 \\
\hline $\mathrm{d}$ & 0.0680 & 0.0940 & 0.0330 & 0.0990 & 0.0500 \\
\hline \multicolumn{6}{|c|}{ Results (05) mm } \\
\hline \multirow[t]{4}{*}{ Fibre no: } & 1 & 2 & 3 & 4 & 5 \\
\hline & 0.0737 & -0.6947 & 0.3687 & 0.3188 & -0.3383 \\
\hline & 0.5813 & 0.4133 & 0.3962 & 0.6172 & 0.6337 \\
\hline & 0.0668 & 0.0926 & 0.0326 & 0.0984 & 0.0510 \\
\hline \multicolumn{6}{|c|}{ Error $=6.3597 \mathrm{e}-017$} \\
\hline
\end{tabular}

Table 4.3 MUAP simulation and decomposition for 5 fibres. 

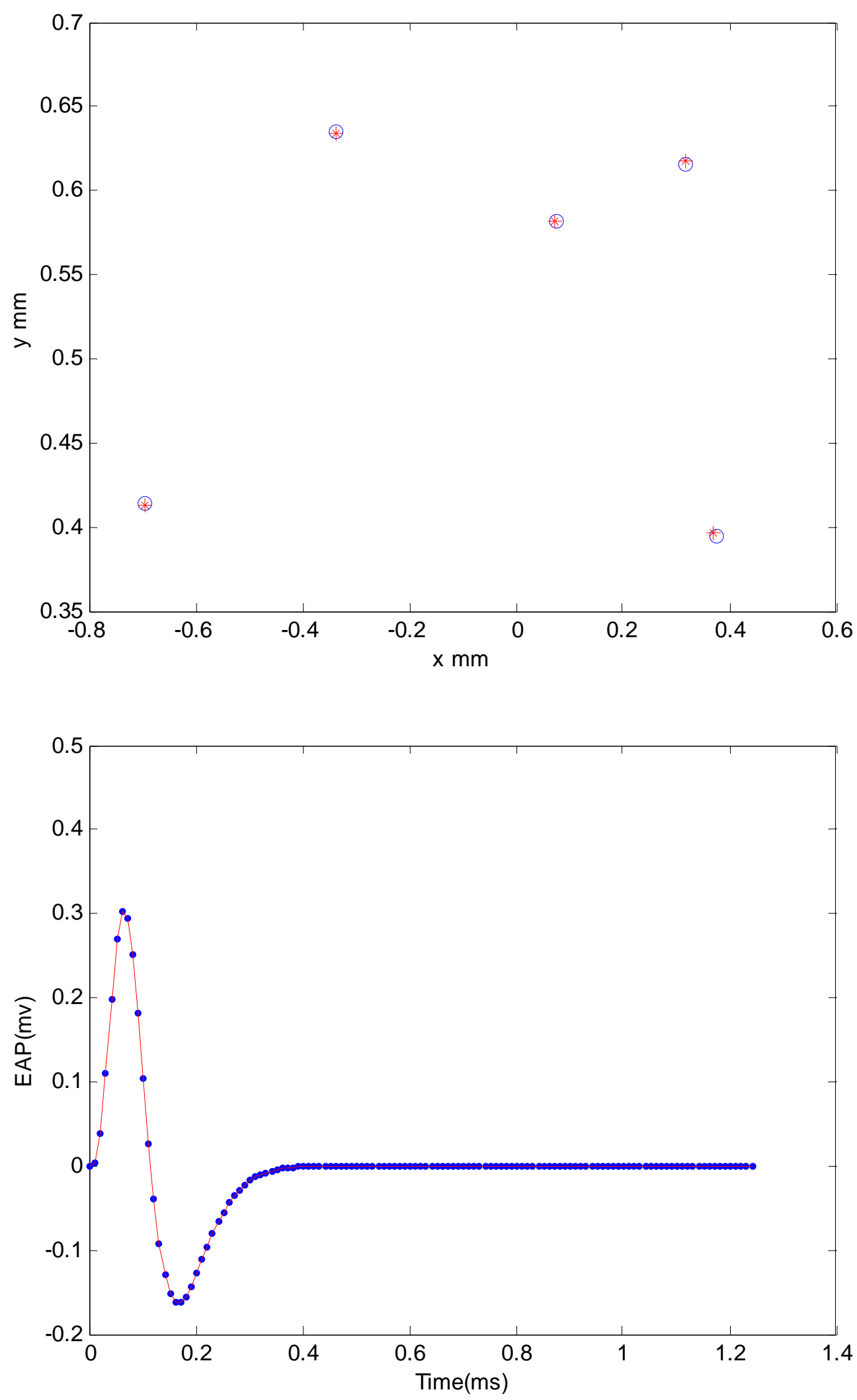

Fig. 4.3 Simulation and decomposition of 5 fibres for a test radius of $1.0 \mathrm{~mm}$. (simulated blue '.', forecasted red '-')

The top graph shows the positioning of the fibres.

(simulated blue 'o', forecasted red '+') 


\subsubsection{MUAP simulation for 10 fibres}

Table 4.4 gives the simulated, initial and final results for the $x$ and $y$ coordinates and diameter, $d$. It is clearly shown that the final results are very close to the simulated values in 97 iterations with an infinitely small error. Figure 4.4 illustrates clearly the overlap of the coordinates between the simulated and final values as well as the overlap between the simulated and final waveforms.

\begin{tabular}{|c|c|c|c|c|c|c|c|c|c|c|}
\hline \multicolumn{11}{|c|}{ Simulated values (r10) mm } \\
\hline Fibre no: & 1 & 2 & 3 & 4 & 5 & 6 & 7 & 8 & 9 & 10 \\
\hline $\mathrm{x}$ & 0.0751 & -0.6965 & -0.7989 & -0.7652 & 0.7562 & 0.3750 & 0.3180 & 0.8476 & -0.3367 & 0.4152 \\
\hline $\mathrm{y}$ & 0.5817 & 0.4142 & 0.1500 & 0.7031 & 0.0350 & 0.3946 & 0.6158 & 0.0399 & 0.6348 & 0.8204 \\
\hline $\mathrm{d}$ & 0.0679 & 0.0935 & 0.0092 & 0.0654 & 0.0262 & 0.0328 & 0.0983 & 0.0730 & 0.0478 & 0.0238 \\
\hline \multicolumn{11}{|c|}{ Initial Values (z10) mm } \\
\hline Fibre no: & 1 & 2 & 3 & 4 & 5 & 6 & 7 & 8 & 9 & 10 \\
\hline $\mathrm{x}$ & 0.0750 & -0.6960 & -0.7990 & -0.7650 & 0.7560 & 0.3755 & 0.3200 & 0.8500 & -0.3370 & 0.4150 \\
\hline $\mathrm{y}$ & 0.5800 & 0.4140 & 0.1510 & 0.7030 & 0.0360 & 0.3950 & 0.6160 & 0.0400 & 0.6350 & 0.8200 \\
\hline d & 0.0680 & 0.0930 & 0.0090 & 0.0660 & 0.0260 & 0.0330 & 0.0990 & 0.0730 & 0.0480 & 0.0240 \\
\hline \multicolumn{11}{|c|}{ Results (o10) mm } \\
\hline Fibre no: & 1 & 2 & 3 & 4 & 5 & 6 & 7 & 8 & 9 & 10 \\
\hline $\mathrm{x}$ & 0.0750 & -0.6960 & -0.7990 & -0.7650 & 0.7560 & 0.3755 & 0.3200 & 0.8499 & -0.3370 & 0.4150 \\
\hline $\mathrm{y}$ & 0.5800 & 0.4141 & 0.1510 & 0.7030 & 0.0360 & 0.3950 & 0.6160 & 0.0400 & 0.6350 & 0.8200 \\
\hline $\mathrm{d}$ & 0.0677 & 0.0929 & 0.0089 & 0.0662 & 0.0261 & 0.0329 & 0.0984 & 0.0730 & 0.0479 & 0.0238 \\
\hline
\end{tabular}

Table 4.4 MUAP simulation and decomposition for 10 fibres. 

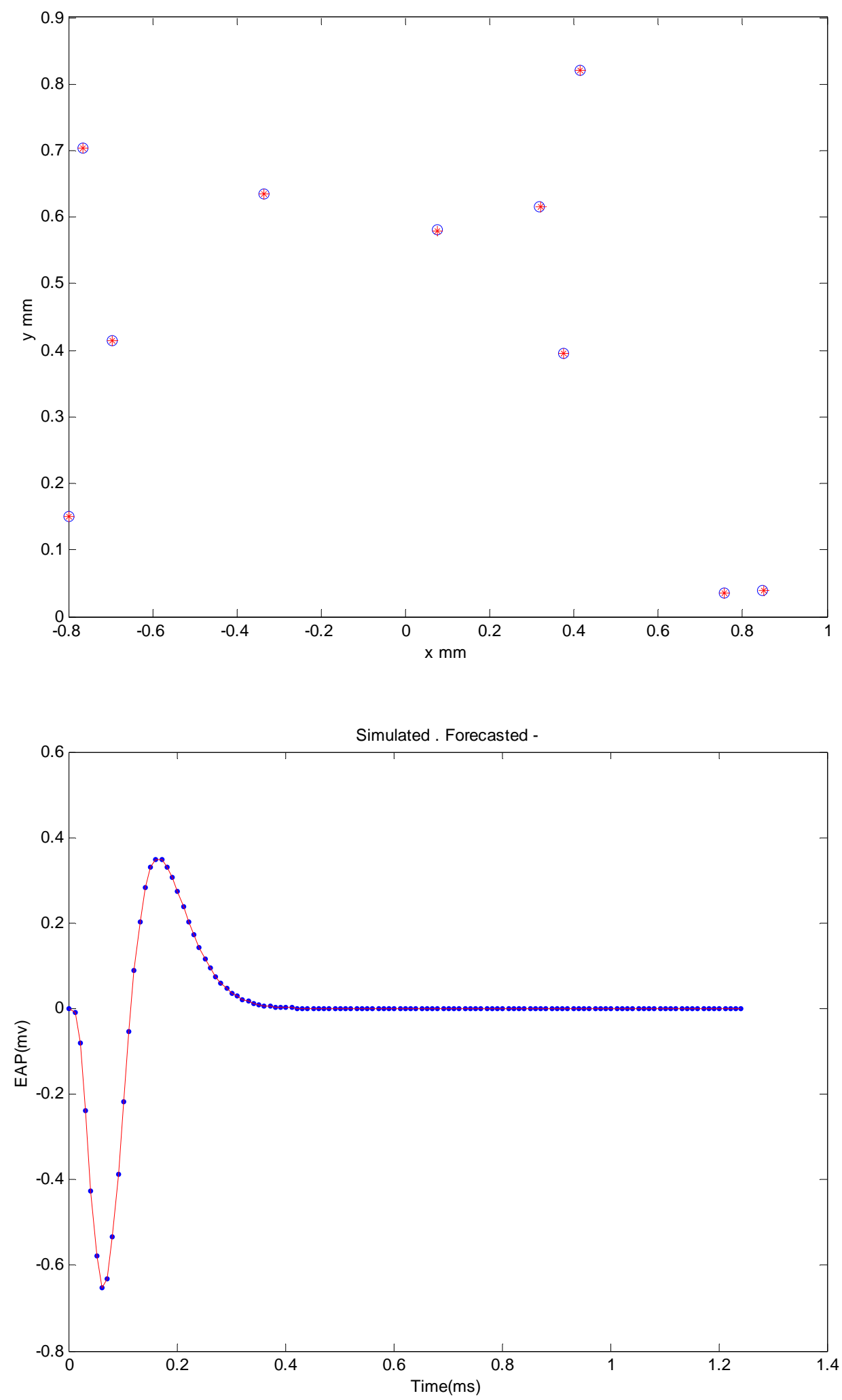

Fig. 4.4 Simulation and decomposition of 10 fibres for a test radius of $1.2 \mathrm{~mm}$. (simulated blue '.', forecasted red '-')

The top graph shows the positioning of the fibres.

(simulated blue 'o', forecasted red '+') 


\subsubsection{Summarized results for MUAP simulation using a two-dimensional model}

Table 4.5 summarizes the results for the two dimensional models. From the above results it is shown that the error (equation 3.4) is minimal.

\begin{tabular}{cccc}
\hline No of Fibres & Test radius $(\mathrm{mm})$ & No of Iterations & Error \\
\hline 1 & 0.6 & 17 & $4.7475 \mathrm{e}^{-011}$ \\
4 & 0.9 & 58 & $1.9997 \mathrm{e}^{-013}$ \\
5 & 1.0 & 53 & $6.3597 \mathrm{e}^{-017}$ \\
10 & 1.2 & 97 & $9.0148 \mathrm{e}^{-016}$ \\
\hline
\end{tabular}

Table 4.5 Summarized results for two-dimensional models. 


\subsection{MUAP Simulation and Decomposition Using a Three-Dimensional Model for} Normal MUAPS

MUAP simulations with 50 fibres using a three-dimensional model were carried out. For each experiment initial values, simulated values and forecasted values (results) are given for the $x$-horizontal distance, $y$-vertical distance, $z$-distance, $d$-diameter and $t$ delay. The number of the iterations has been given as a parameter. Plots of the simulated and final waveforms are given. Tables A.3.1 and A.3.2 in Appendix 3 give the simulated values and the initial values respectively. 


\subsubsection{MUAP simulation 1: 50 fibres, number of iterations 251, Error= 8.6774}

Table A.3.3 in Appendix 3 gives the final values (results) for the $x$-horizontal distance, $y$-vertical distance, $z$-distance and $d$-diameter. It is shown that the error is not minimal after 251 iterations. Figure 4.5 illustrates a difference between the simulated and the final waveform.

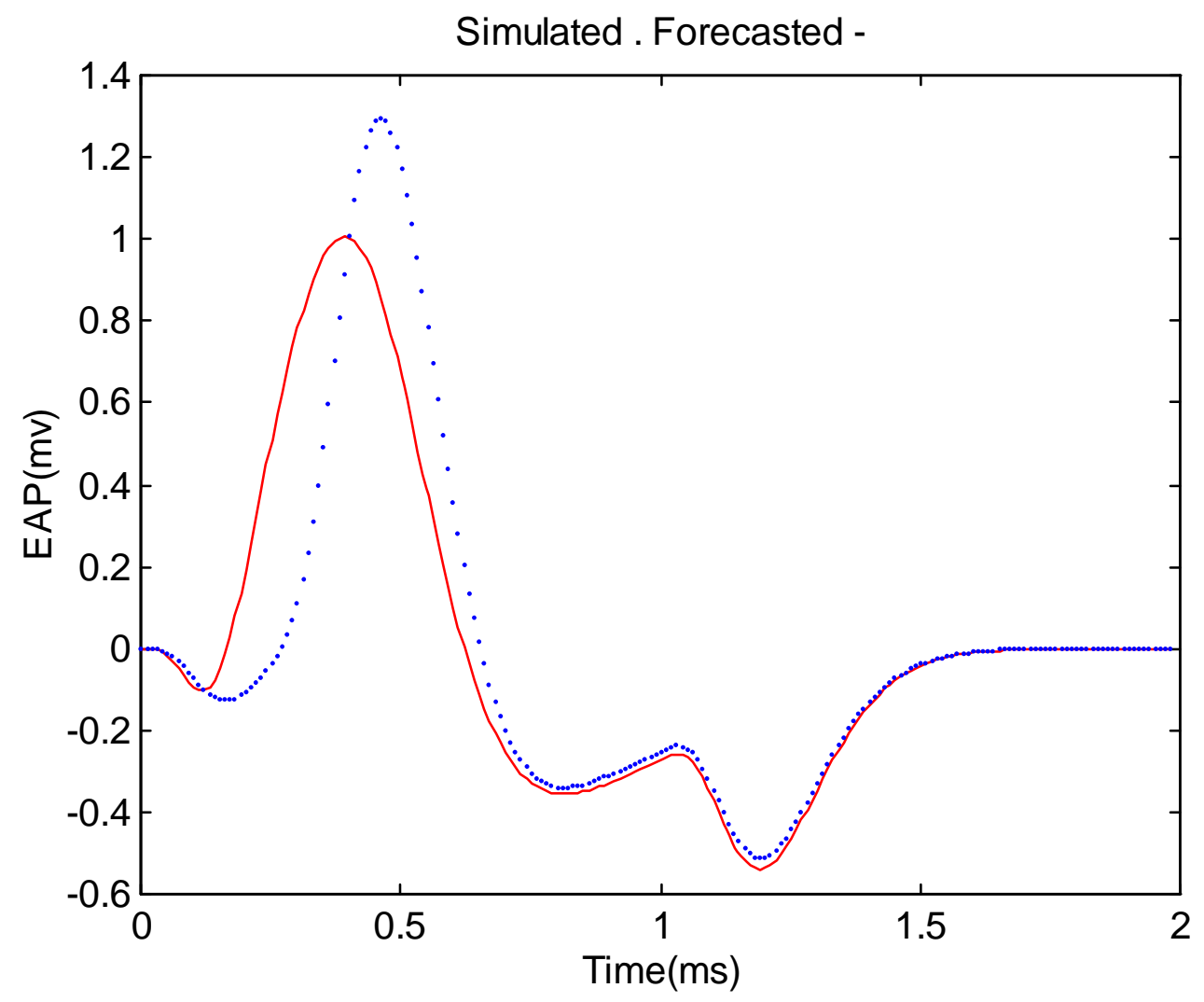

Fig. 4.5 Simulation and decomposition of 50 fibres for a test radius of $5 \mathrm{~mm}$. No of iterations $=251$. (simulated blue '.', forecasted red '-') 


\subsubsection{MUAP simulation 2: 50 fibres, number of iterations 1011, Error=0.0187}

Table A.3.4 in Appendix 3 gives the final values (results) for the $x$-horizontal distance, $y$-vertical distance, $z$-distance and $d$-diameter. It is shown that in 1011 iterations the error is significant smaller than the 251 iterations case but still is not minimal. Figure 4.6 illustrates the overlap between the simulated and final waveform.

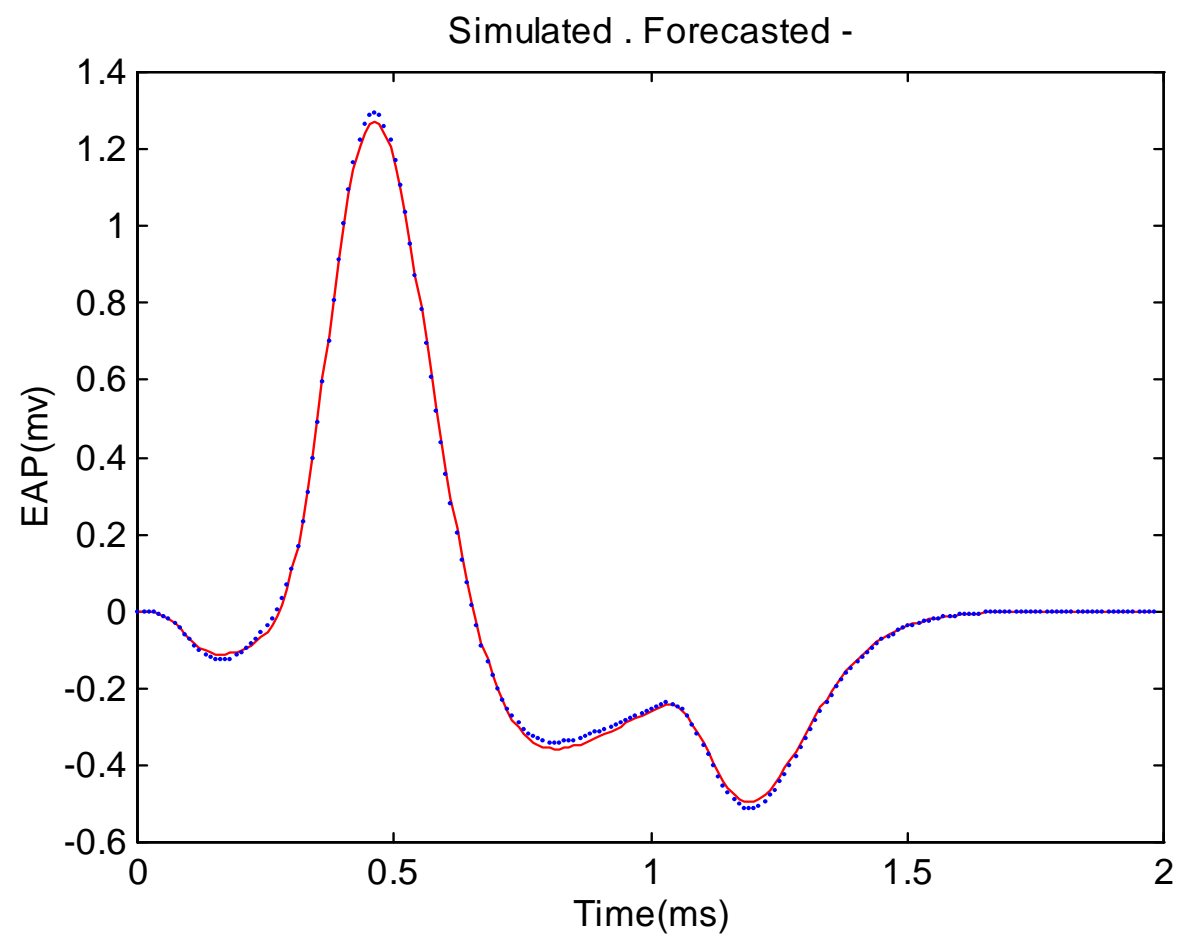

Fig. 4.6 Simulation and decomposition of 50 fibres for a test radius of $5 \mathrm{~mm}$. No of iterations=1011. (simulated blue '.', forecasted red '-') 


\subsubsection{MUAP simulation 3: 50 fibres, number of iterations 5065, Error=2.7241e-008}

Table A.3.5 in Appendix 3 gives the final values (results) for the $x$-horizontal distance, $y$-vertical distance, $z$-distance and $d$-diameter. In this case the final values (results) obtained by the previous experiment were used as initial. It is shown that the error is infinitely small after 5065 iterations. Figure 4.7 illustrates the closeness of the coordinates between the simulated and the final values as well as the overlap between the simulated and the final waveform. 


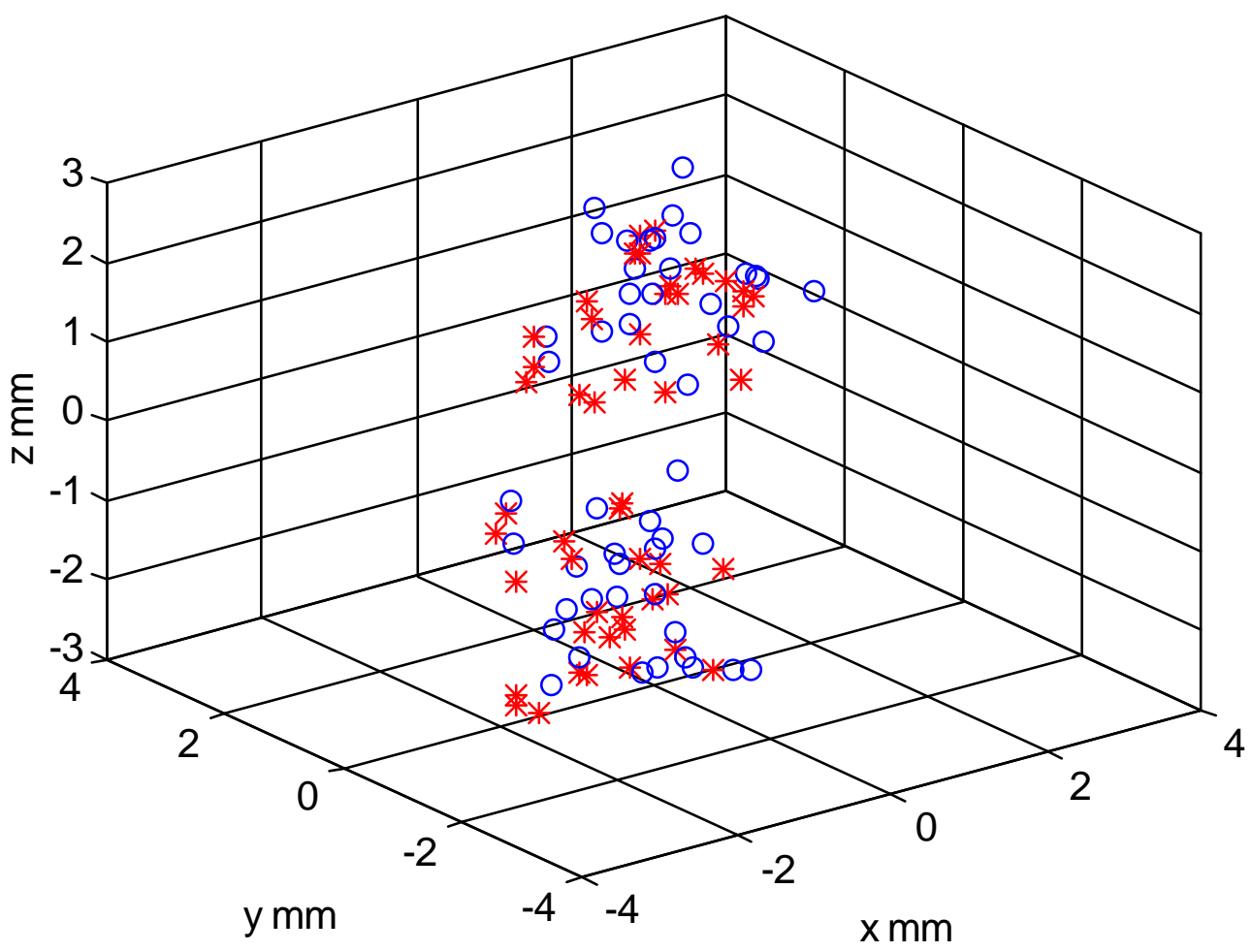

Simulated . Forecasted -

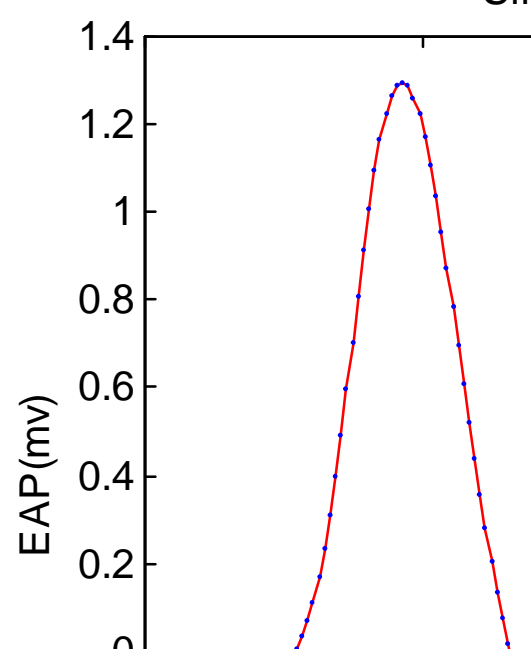

Fig. 4.7 Simulation and decomposition of 50 fibres for a test radius of $5 \mathrm{~mm}$. No of iterations $=5065$. ( simulated blue '.', forecasted red '-') The top graph shows the positioning of the fibres. (simulated blue 'o', forecasted red ' + ') 


\subsubsection{Summarized results for normal MUAP simulation using a three-dimensional} model

Table 4.6 tabulates the summarized results for a normal MUAP based on the threedimensional model. It is shown that the error is infinitely small in 5065 iterations.

\begin{tabular}{|c|c|c|c|}
\hline No of Fibres & Test radius $(\mathrm{mm})$ & No of Iterations & Error \\
\hline 50 & 5 & 251 & 8.6774 \\
\hline 50 & 5 & 1011 & 0.0187 \\
\hline 50 & 5 & 5065 & $2.7241 \mathrm{e}-008$ \\
\hline
\end{tabular}

Table 4.6 Summarized results for normal MUAP simulation using a three-dimensional model. 


\subsection{MUAP Simulation and Decomposition Using a Three-Dimensional Model for Myopathy}

MUAP simulations with 50 and 75 fibres using a three-dimensional model have been carried out for myopathic cases. For each experiment simulated values and forecasted values (results) are given for the $x$-horizontal distance, $y$-vertical distance, $z$-distance, $d$ diameter and $t$-delay. The number of the iterations has been given as a parameter. Plots of the simulated and final waveforms are given also. Three different cases of myopathic MUAPS are examined:

\section{A. $\quad$ Loss of half of the fibres (Simulation 1-3)}

It has been simulated that half of the fibres were lost from both planes in the MU's territory. Table A.4.1 in Appendix 4 gives the simulated values for the fibres remained (20 to 44) for the $x$-horizontal distance, $y$-vertical distance, $\mathrm{z}$-distance, d-diameter and $\mathrm{t}$ delay. Table A.4.2 in Appendix 4 gives the initial values used.

\section{B. $\quad$ Variability in the diameter (Simulation 4-6)}

Table A.4.6 in Appendix 4 gives the simulated values with a change in diameter (the diameter is twice as big as the diameter in normal cases). Table A.3.2 in Appendix 3 gives the initial values used, which are the same as in the case of the normal MUAP.

\section{Reinnervation of fibres (Simulation 7-12)}

Two cases of reinnervation of fibres are examined. In the first case 25 fibres have been added in both planes in MU's territory (simulation 7-9) and the simulated values are given in Table A.4.10 in Appendix 4. In the second case, 25 fibres have been added in the right plane of MU's territory (simulation 10-12) and the simulated values are given in Tables A.4.15 in Appendix 4. Tables A.4.11 and A.4.16 in Appendix 4 give the initial values used respectively. 


\subsubsection{MUAP myopathy simulation 1, loss of half of the fibres: number of iterations} 221, Error $=0.3823$

Table A.4.3 in Appendix 4 gives the final values (results) for the $x$-horizontal distance, $y$-vertical distance, $z$-distance and $d$-diameter. It is shown that the error is not minimal after 221 iterations. Figure 4.8 illustrates the difference between the simulated and the final waveform.

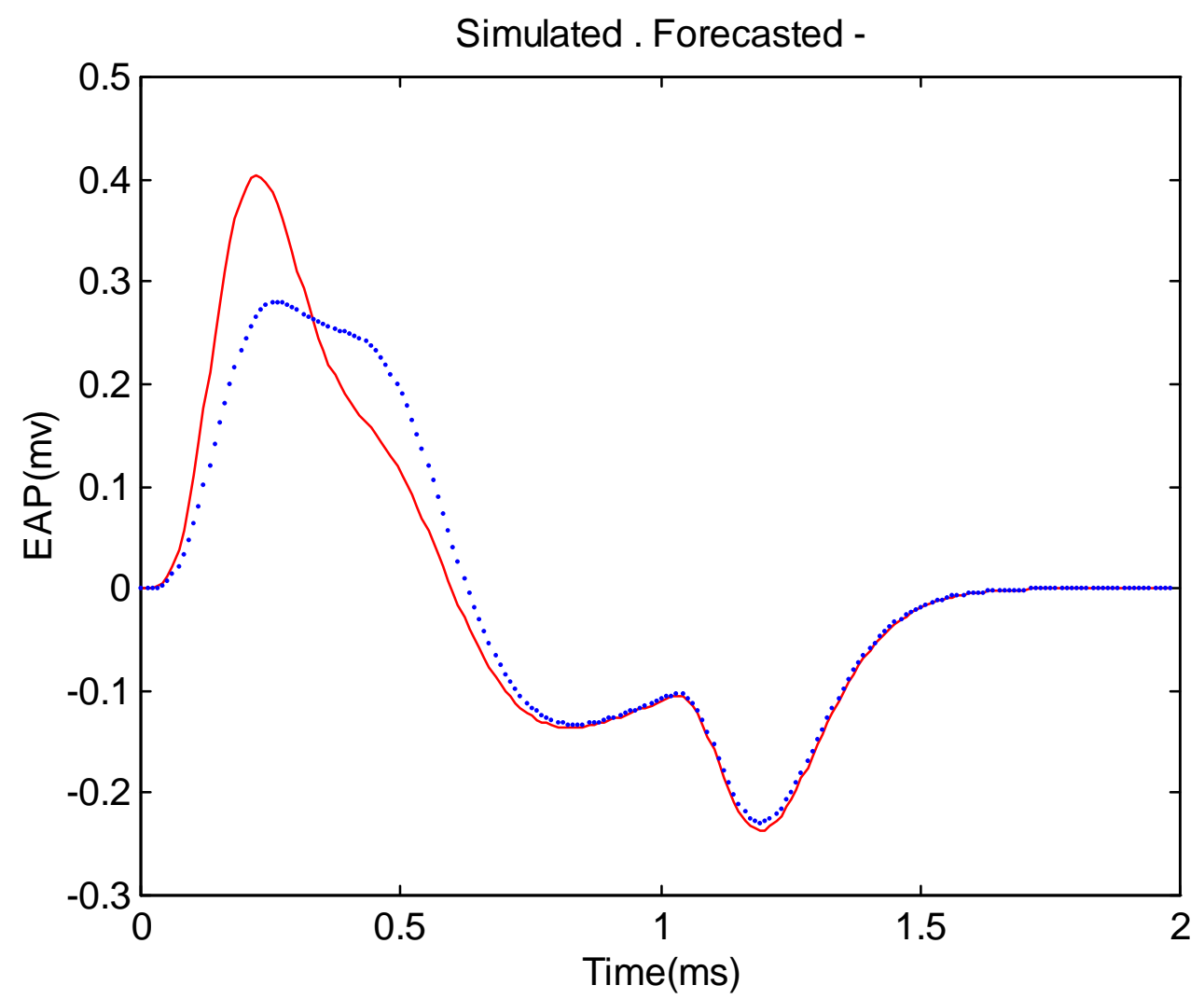

Fig. 4.8 Loss of half of the fibres. Simulation and decomposition of 25 fibres for a test radius of $5 \mathrm{~mm}$. No of iterations=221. (simulated blue '.', forecasted red '-') 


\subsubsection{MUAP myopathy simulation 2, loss of half of the fibres: number of iterations}

\section{5, Error $=2.0846 e-005$}

Table A.4.4 in Appendix 4 gives the final values (results) for the $x$-horizontal distance, $y$-vertical distance, $z$-distance and $d$-diameter. It is shown that the error is minimal after 1115 iterations. Figure 4.9 illustrates the overlap between the simulated and the final waveform.

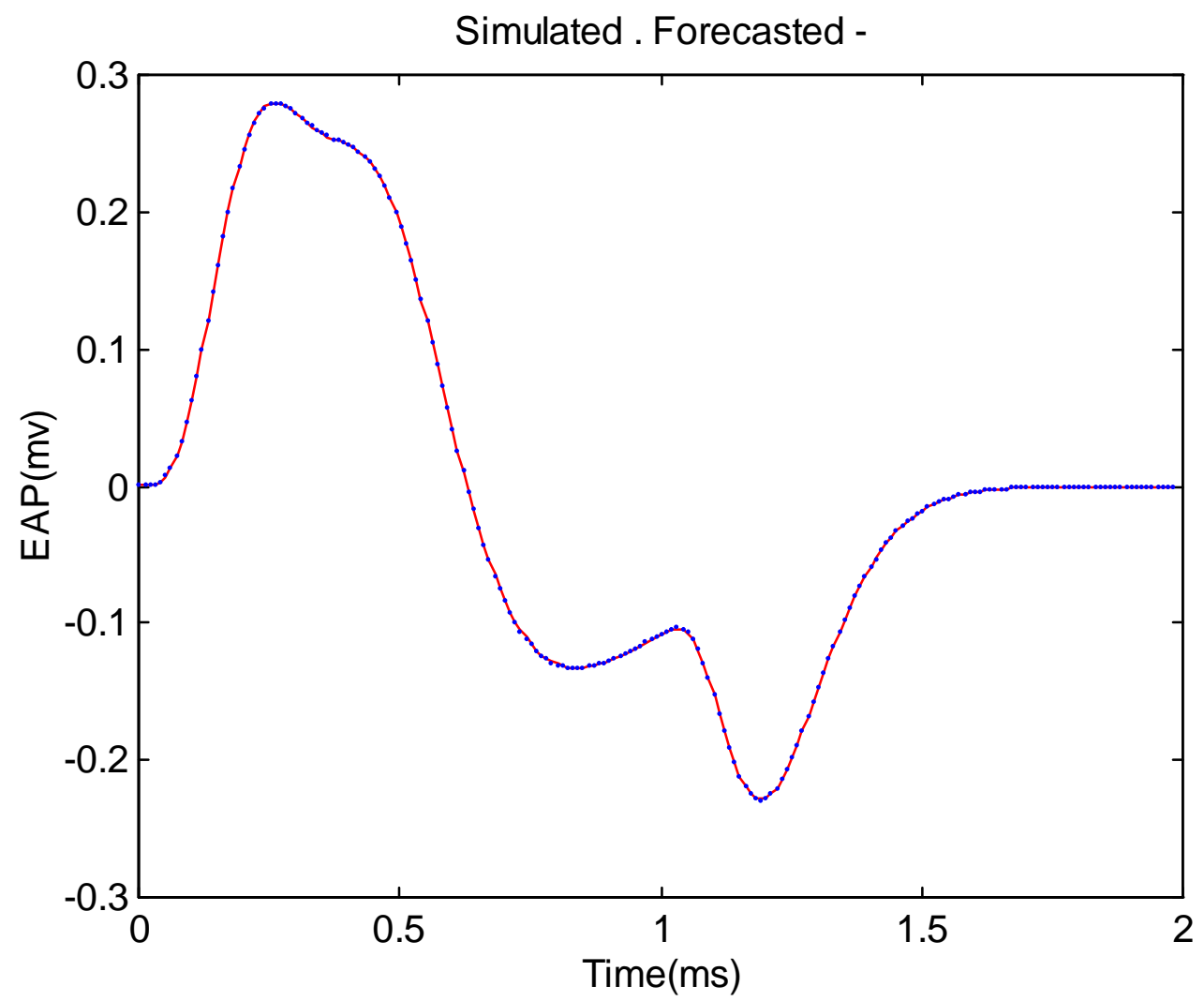

Fig. 4.9 Loss of half of the fibres. Simulation and decomposition of 25 fibres for a test radius of $5 \mathrm{~mm}$. No of Iterations $=1115$. ( 


\subsubsection{MUAP myopathy simulation 3, loss of half of the fibres: number of iterations} 1113, Error $=3.8031 e-007$

Table A.4.5 in Appendix 4 gives the final values (results) for the $x$-horizontal distance, $y$-vertical distance, $z$-distance and $d$-diameter. In this experiment the final values (results) of the previous experiment were used as initial values. As we can see the error is almost infinitely small. Figure 4.10 illustrates the closeness of the coordinates between the simulated and final values as well as the overlap between the simulated and final waveform. 


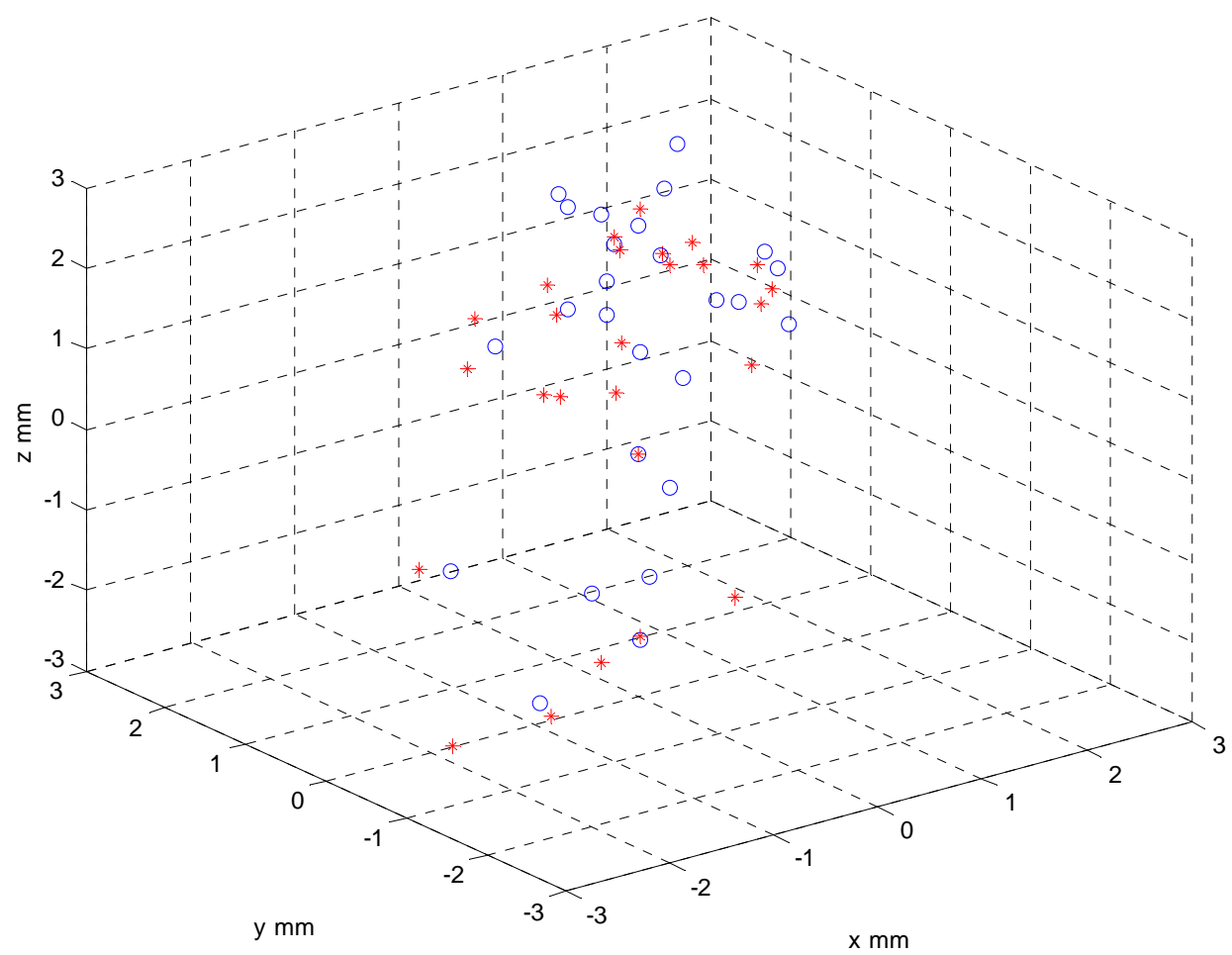

Simulated . Forecasted -

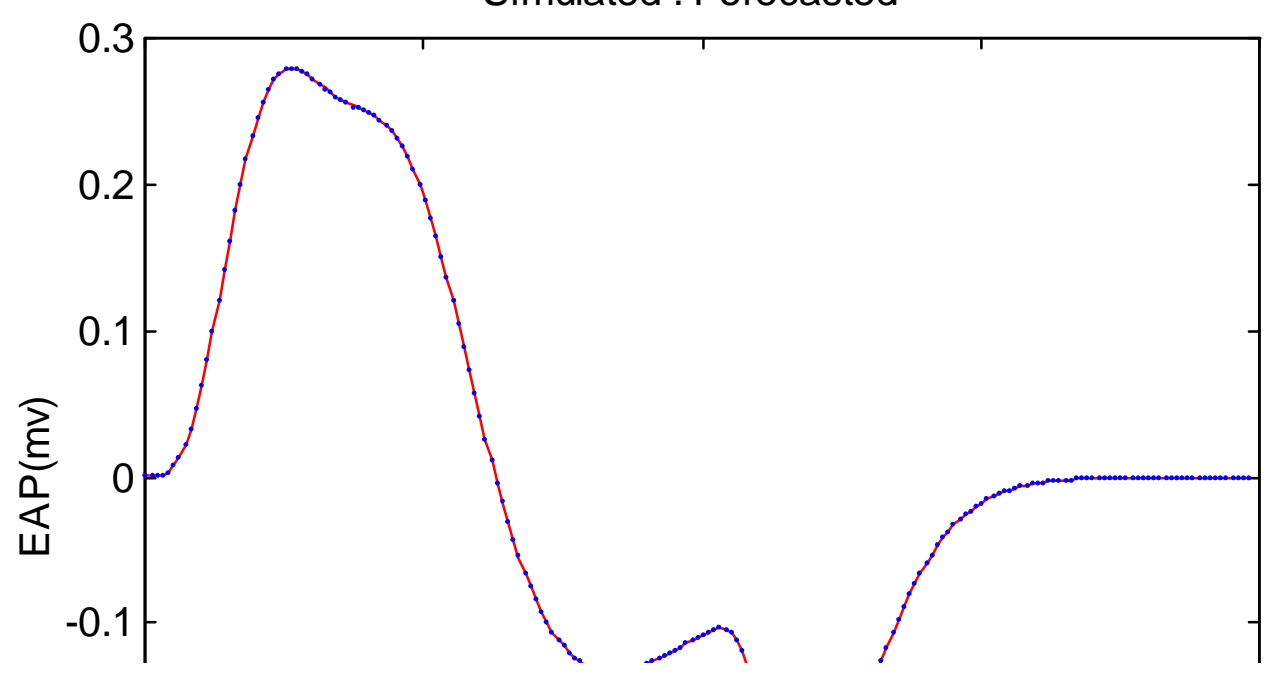

Fig. 4.10 Loss of half of the fibres. Simulation and decomposition of 25 fibres for a test radius of $5 \mathrm{~mm}$. No of iterations=1113. (simulated blue '.', forecasted red '-').

The top graph shows the positioning of the fibres. (simulated blue 'o', forecasted red '+') 


\subsubsection{MUAP myopathy simulation 4, variability in diameter: number of iterations}

\section{1, Error=346.1319}

Table A.4.7 in Appendix 4 gives the final values (results) for the $x$-horizontal distance, $y$-vertical distance, $z$-distance and $d$-diameter. It is shown that the algorithm didn't converge after 251 iterations. Figure 4.11 illustrates the difference between the simulated and final waveform.

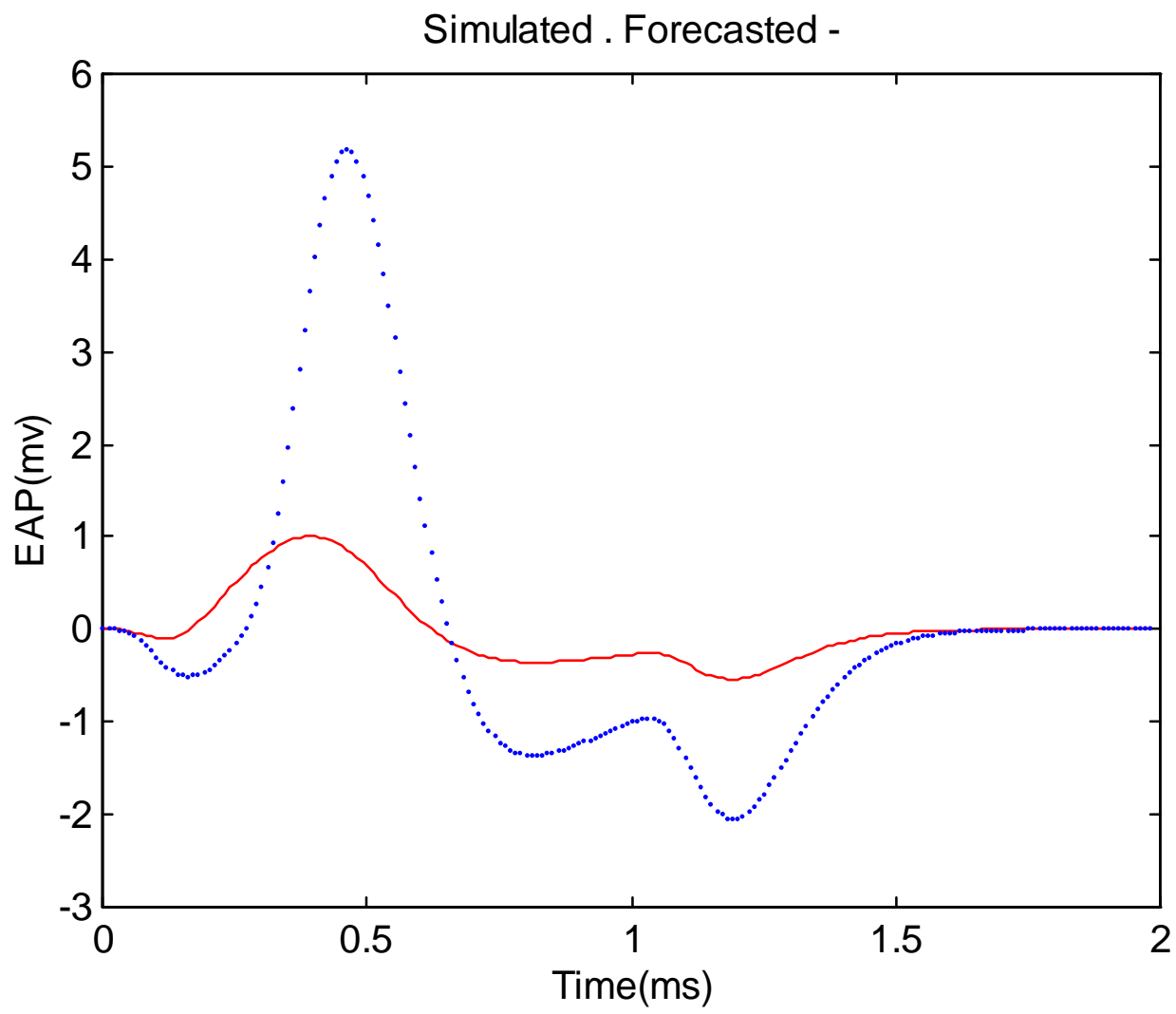

Fig. 4.11 Variability in diameter. Simulation and decomposition of 50 fibres for a test radius of $5 \mathrm{~mm}$. No of iterations $=251$. (simulated blue '.', forecasted red '-') 


\subsubsection{MUAP myopathy simulation 5, variability in diameter: number of iterations} 1011, Error=5.9174.

Table A.4.8 in Appendix 4 gives the final values (results) for the $x$-horizontal distance, $y$-vertical distance, $z$-distance and $d$-diameter. It is shown that the error is not minimal after 1011 iterations. The algorithm executed twice with the same options and the previous values were used as input. Figure 4.12 illustrates the difference between the simulated and final waveform.

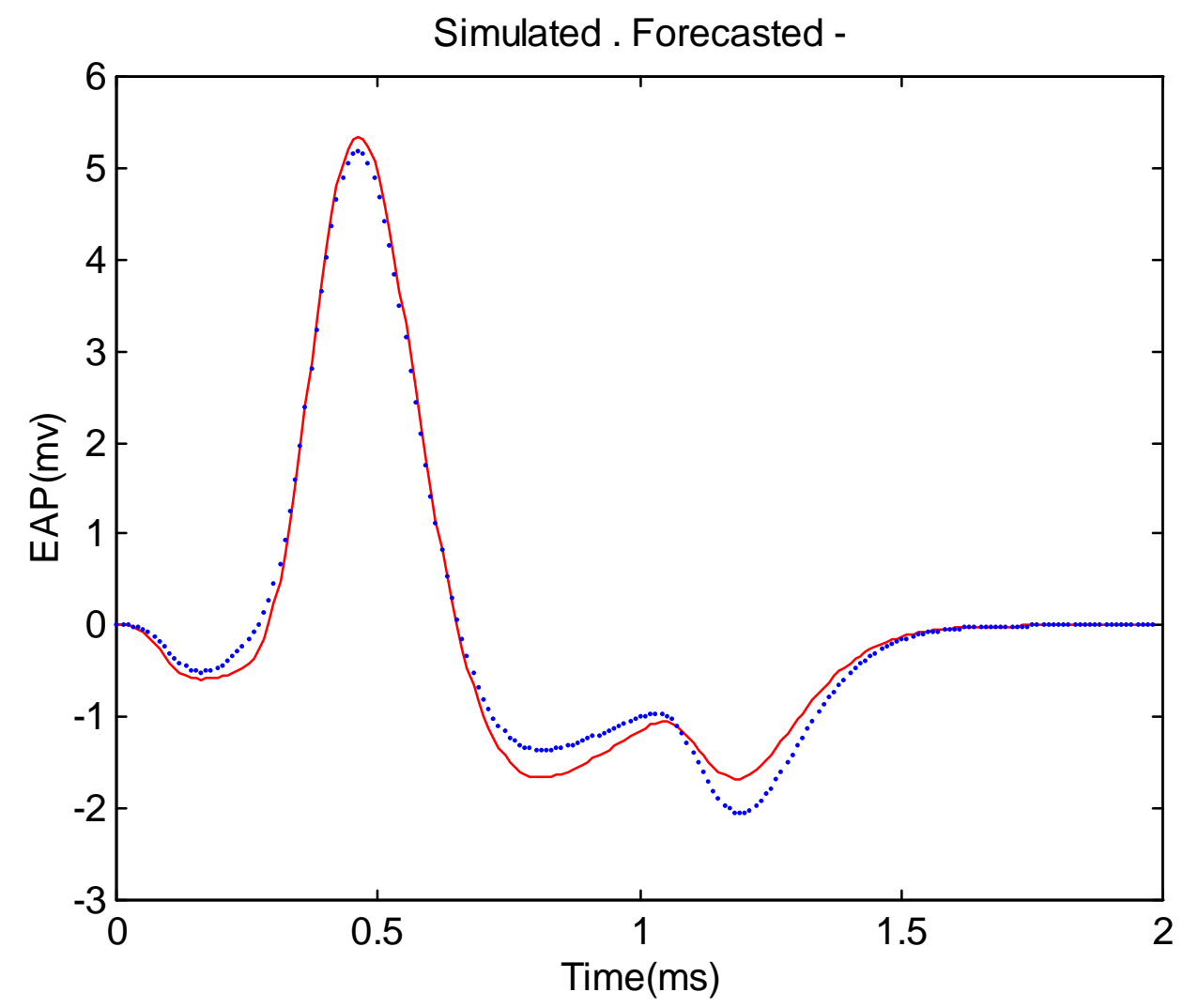

Fig. 4.12. Variability in diameter. Simulation and decomposition of 50 fibres for a test radius of $5 \mathrm{~mm}$. No of iterations $=1011$. (simulated blue '.', forecasted red '-') 


\subsubsection{MUAP myopathy simulation 6, variability in diameter: number of iterations} 5066, Error $=4.1195 \mathrm{e}-004$

Table A.4.9 in Appendix 4 gives the final values (results) for the $x$-horizontal distance, $y$-vertical distance, $z$-distance and d-diameter. It is shown that the error is minimal after 5066 iterations. The previous results were used as input. Figure 4.13 illustrates the closeness of the coordinates between the simulated and final fibres as well as the overlap between the simulated and final waveform. 


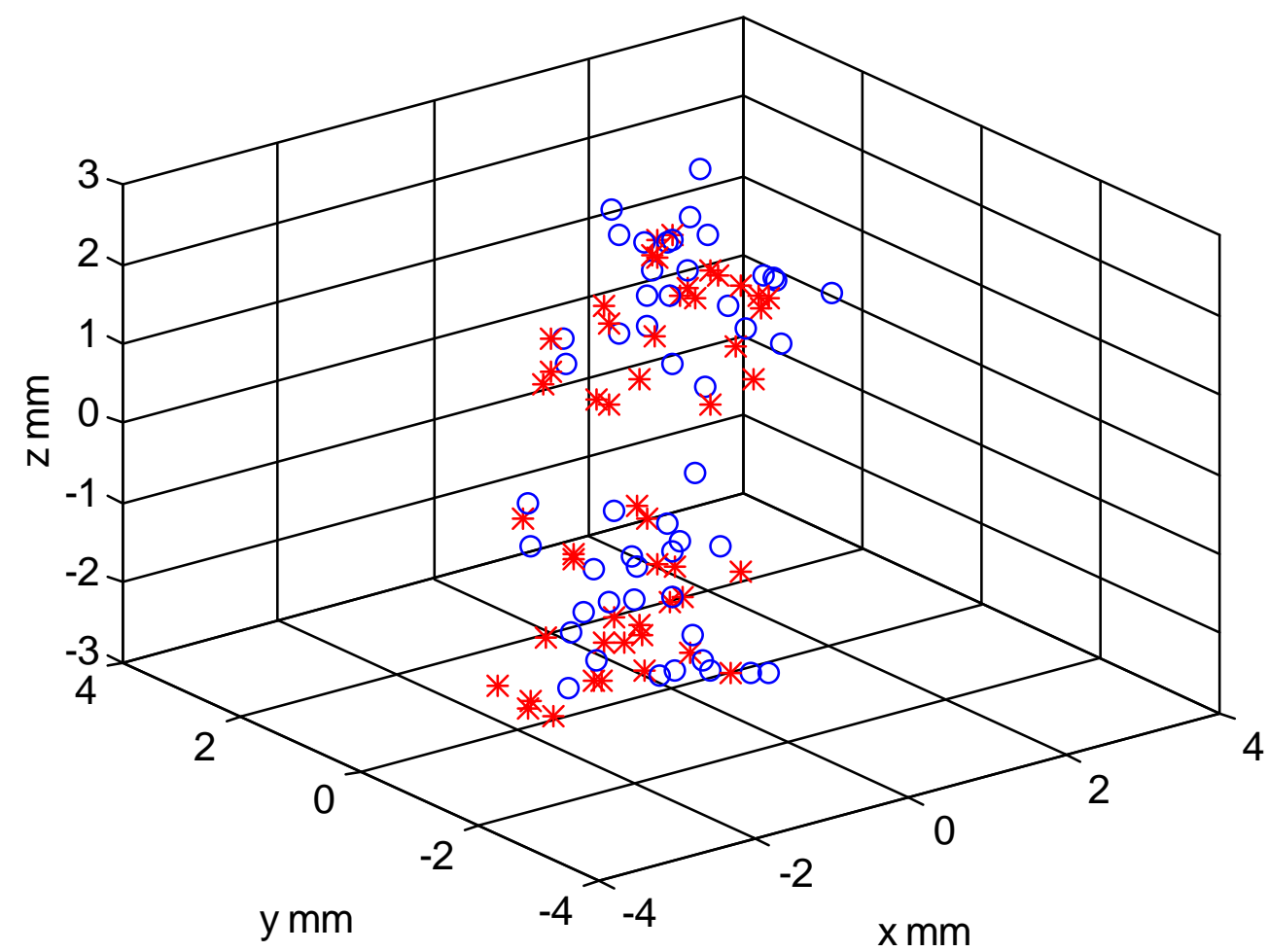

Simulated . Forecasted -
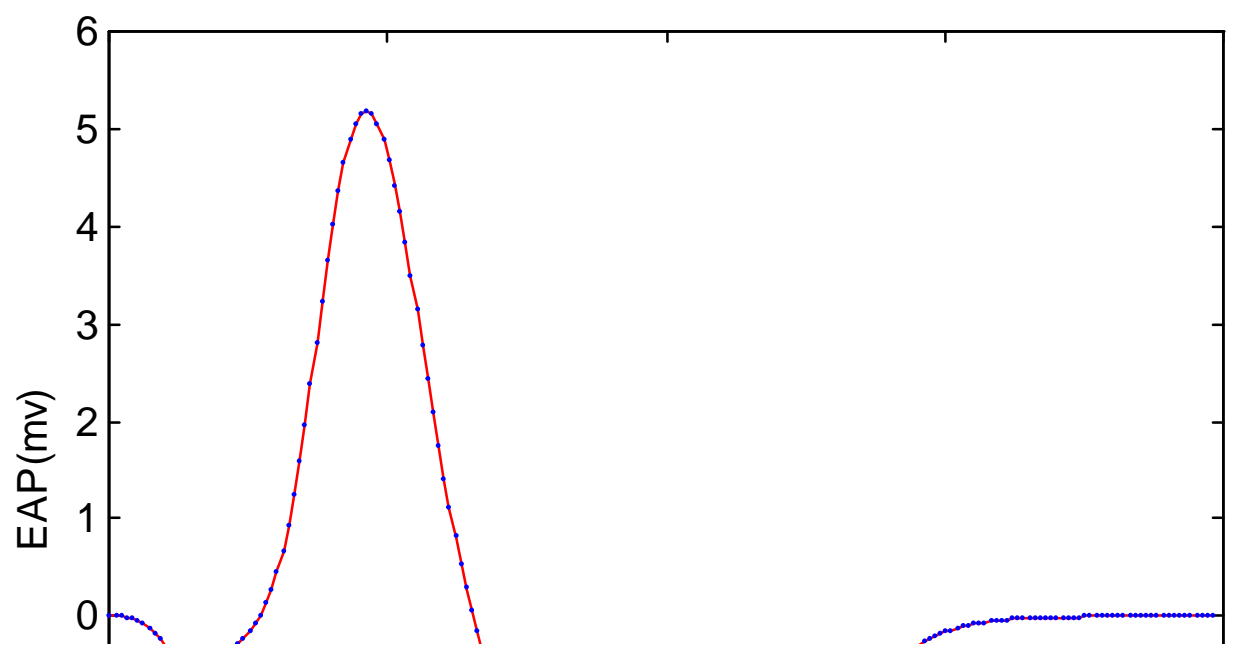

Fig. 4.13. Variability in diameter. Simulation and decomposition of 50 fibres for a test radius of $5 \mathrm{~mm}$. No of iterations $=5066$. ( simulated blue ' , forecasted red '-')

The top graph shows the positioning of the fibres. (simulated blue 'o', forecasted red ' + ') 


\subsubsection{MUAP myopathy simulation 7, reinnervation of 25 fibres in both planes of} the MU territory: number of iterations 376, Error $=6.3581$

Table A.4.12 in Appendix 4 gives the final values (results) for the $x$-horizontal distance, $y$-vertical distance, $z$-distance and $d$-diameter. It is shown that the algorithm does not converge after 376 iterations. Figure 4.14 illustrates the difference between the simulated and final waveform.

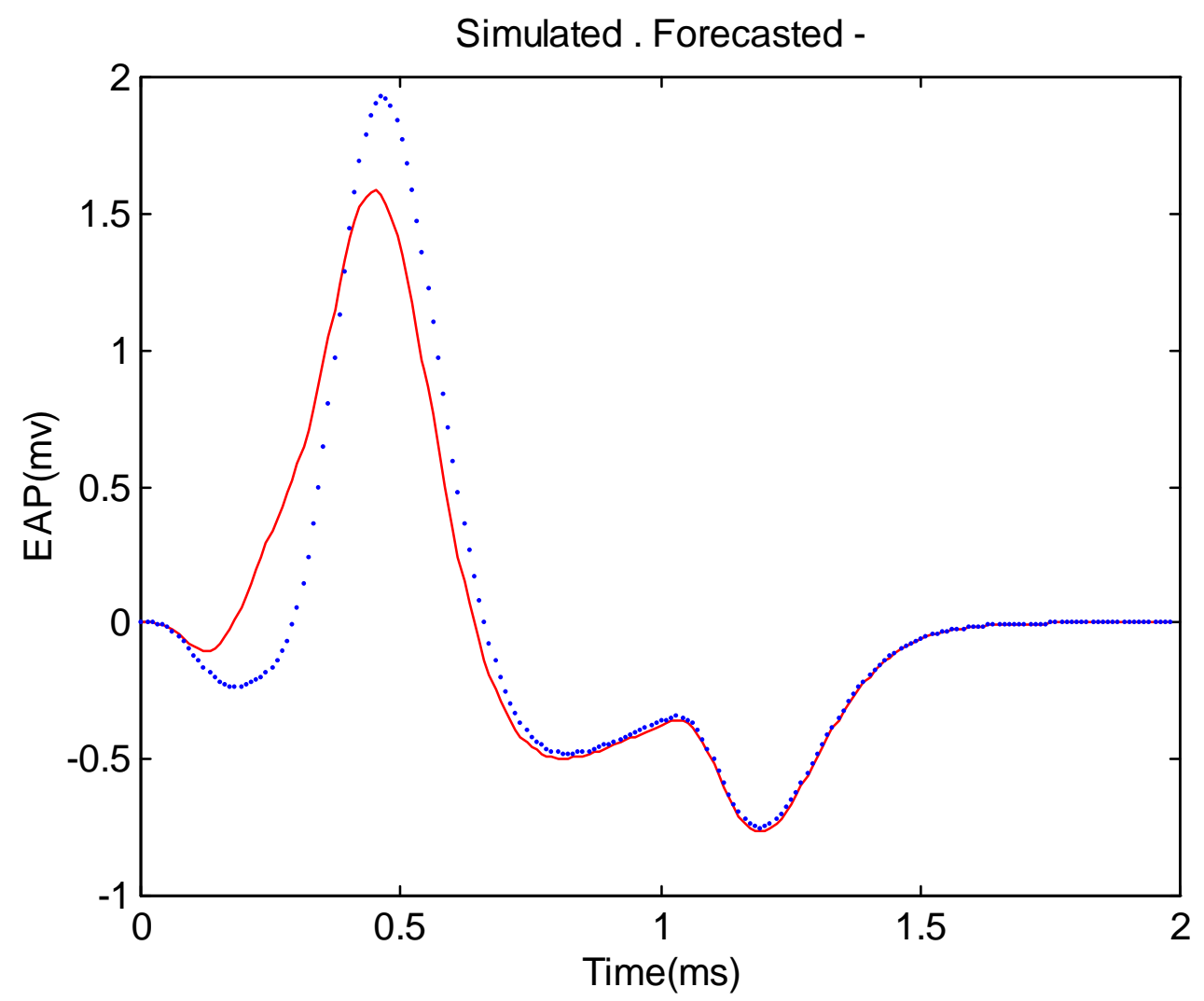

Fig. 4.14 Reinnervation of fibres. Simulation and decomposition of 75 fibres for a test radius of $5 \mathrm{~mm}$. Twenty five fibres were reinnervated in both planes of the MU territory. No of Iterations $=376$. ( simulated blue '.', forecasted red '-') 


\subsubsection{MUAP myopathy simulation 8, reinnervation of 25 fibres in both planes of} the MU territory: number of iterations 1132, Error $=0.0087$

Table A.4.13 in Appendix 4 gives the final values (results) for the $x$-horizontal distance, $y$-vertical distance, $z$-distance and $d$-diameter. It is shown that the error is smaller than in the case of 376 iterations but still not minimal. Figure 4.15 illustrates the overlap between the simulated and final waveform.

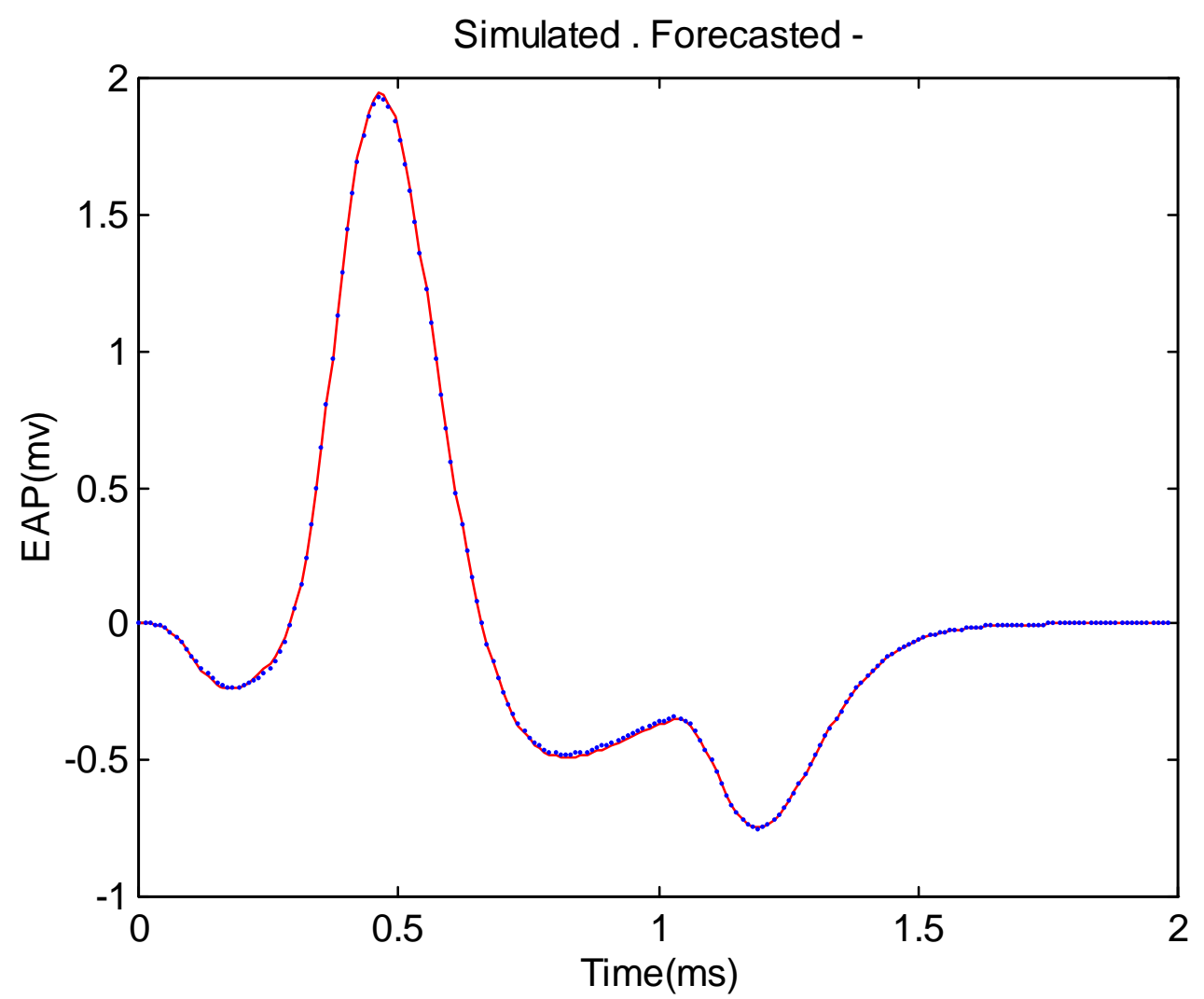

Fig. 4.15 Reinnervation of fibres. Simulation and decomposition of 75 fibres for a test radius of $5 \mathrm{~mm}$. Twenty five fibres were reinnervated in both planes of the MU territory. No of iterations=1132. ( simulated blue '.', forecasted red '-') 


\subsubsection{MUAP myopathy simulation 9, reinnervation of 25 fibres in both planes of} the MU territory: number of iterations 5294, Error=1.7908e-007

Table A.4.14 in Appendix 4 gives the final values (results) for the $x$-horizontal distance, $y$-vertical distance, $z$-distance and $d$-diameter. It is shown that the error is almost infinitely small after 5294 iterations. Figure 4.16 illustrates the closeness of the coordinates between the simulated and final fibres as well as the overlap between the simulated and final waveform. 


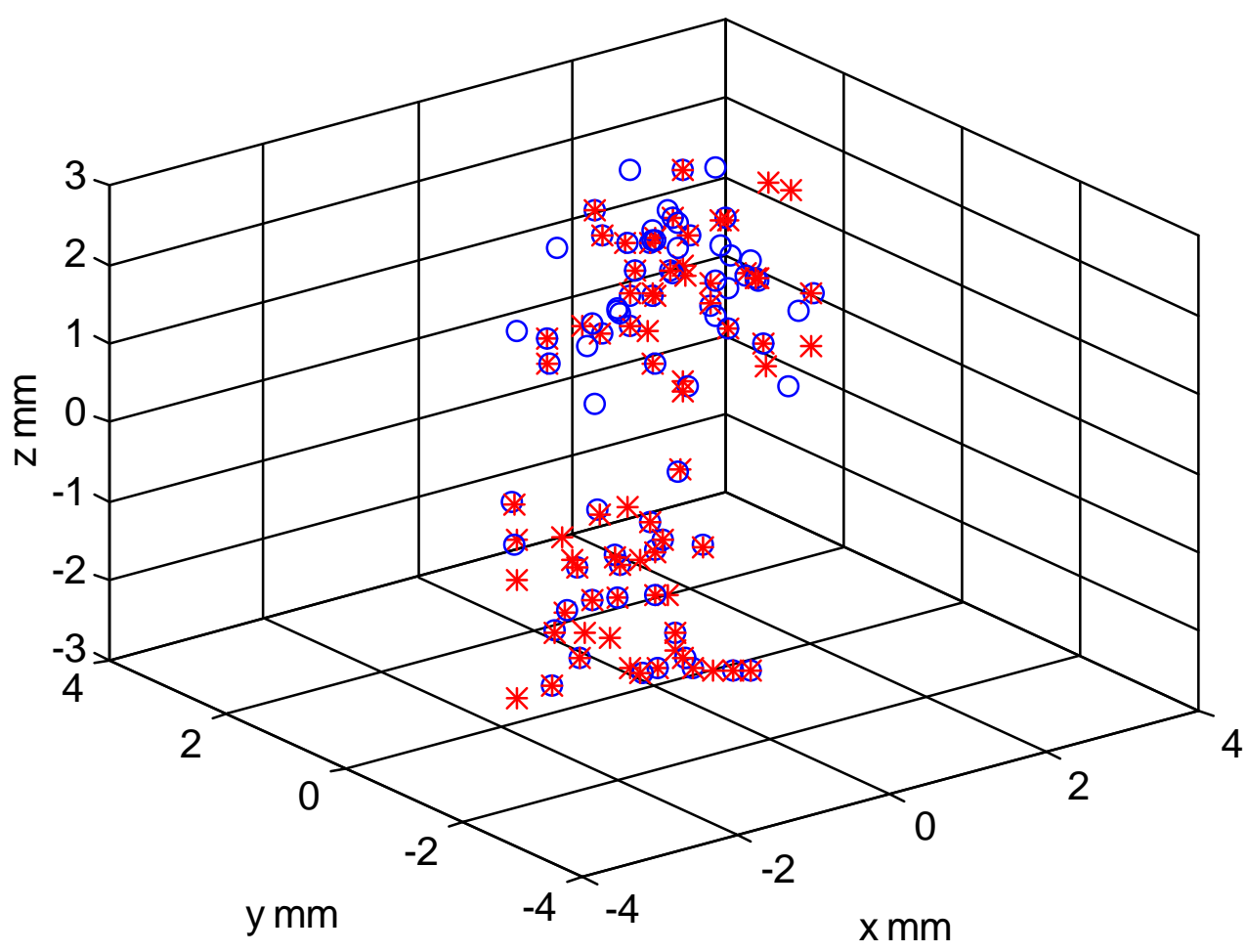

Simulated . Forecasted -
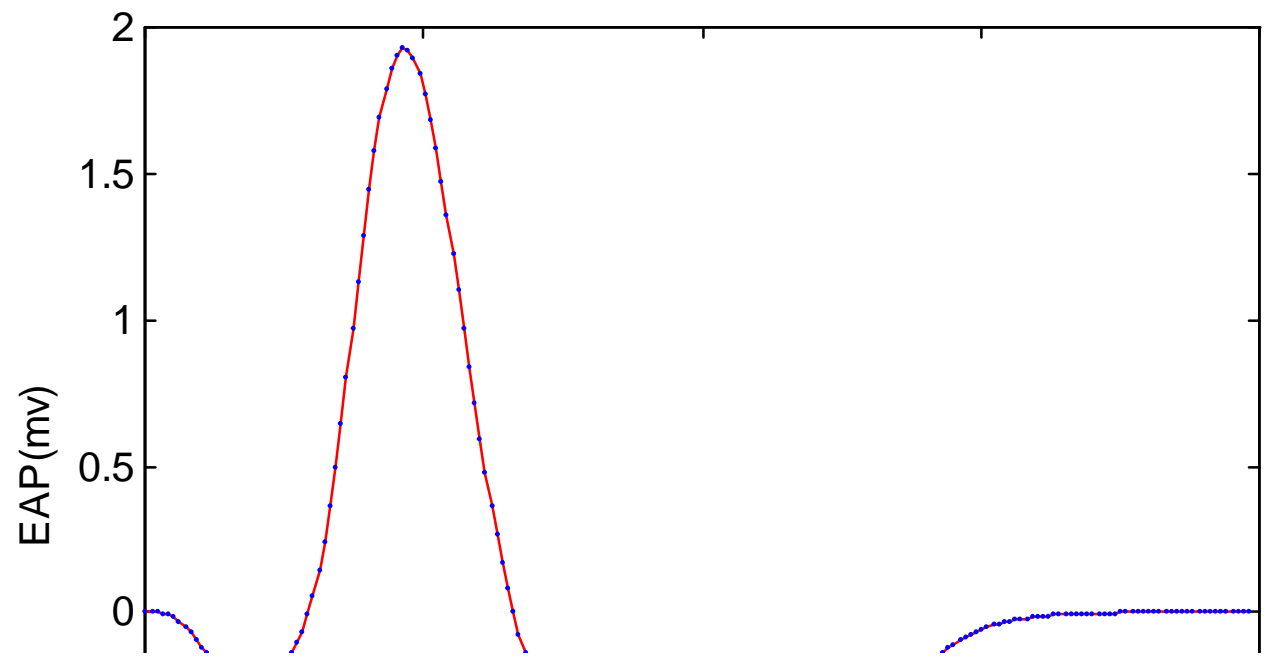

Fig. 4.16 Reinnervation of fibres. Simulation and decomposition of 75 fibres for a test radius of $5 \mathrm{~mm}$. Twenty five fibres were reinnervated in both planes of the MU territory. No of Iterations=5294. (simulated blue '.', forecasted red '-').

The top graph shows the positioning of the fibres. (simulated blue 'o', forecasted red '+') 


\subsubsection{MUAP myopathy simulation 10, reinnervation of 25 fibres in the right plane} of the MU territory: number of iterations 376, Error=3.4393

Table A.4.17 in Appendix 4 gives the values (results) for the $x$-horizontal distance, $y$ vertical distance, $z$-distance and $d$-diameter. It is shown that the algorithm didn't converge after 376 iterations. Figure 4.17 illustrates the difference between the simulated and final waveform.

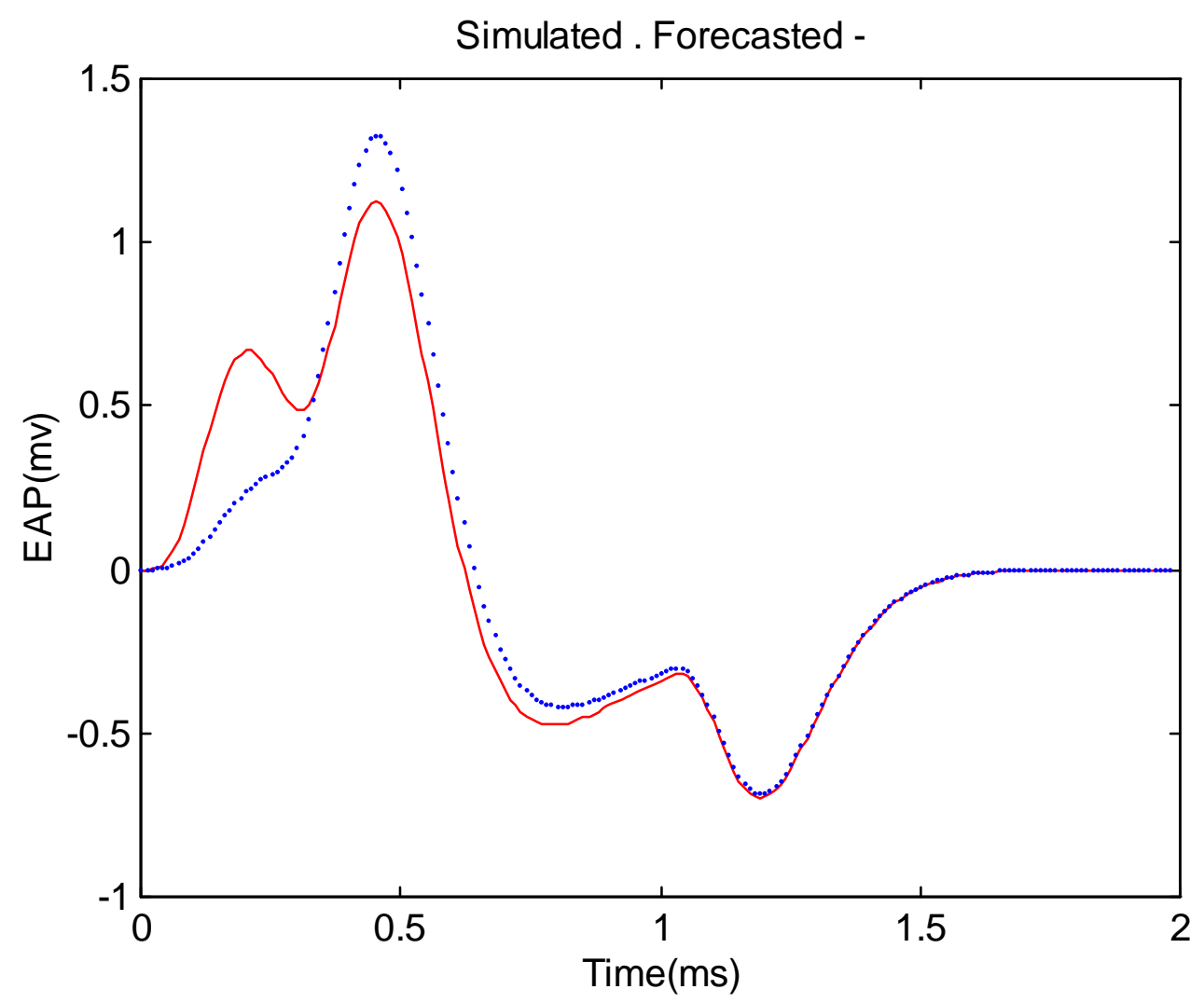

Fig. 4.17 Reinnervation of fibres. Simulation and decomposition of 75 fibres for a test radius of $5 \mathrm{~mm}$. Twenty five fibres were reinnervated in the right plane of the MU territory. No of iterations=376. (simulated blue '.', forecasted red '-‘) 


\subsubsection{MUAP myopathy simulation 11, reinnervation of 25 fibres in the right plane} of the MU territory: number of iterations 1113, Error=0.0012

Table A.4.18 in Appendix 4 gives the final values (results) for the $x$-horizontal distance, $y$-vertical distance, $z$-distance and $d$-diameter. It is shown that the error is smaller than the 376 iterations case but still is not minimal. Figure 4.18 illustrates the difference between the simulated and final waveform.

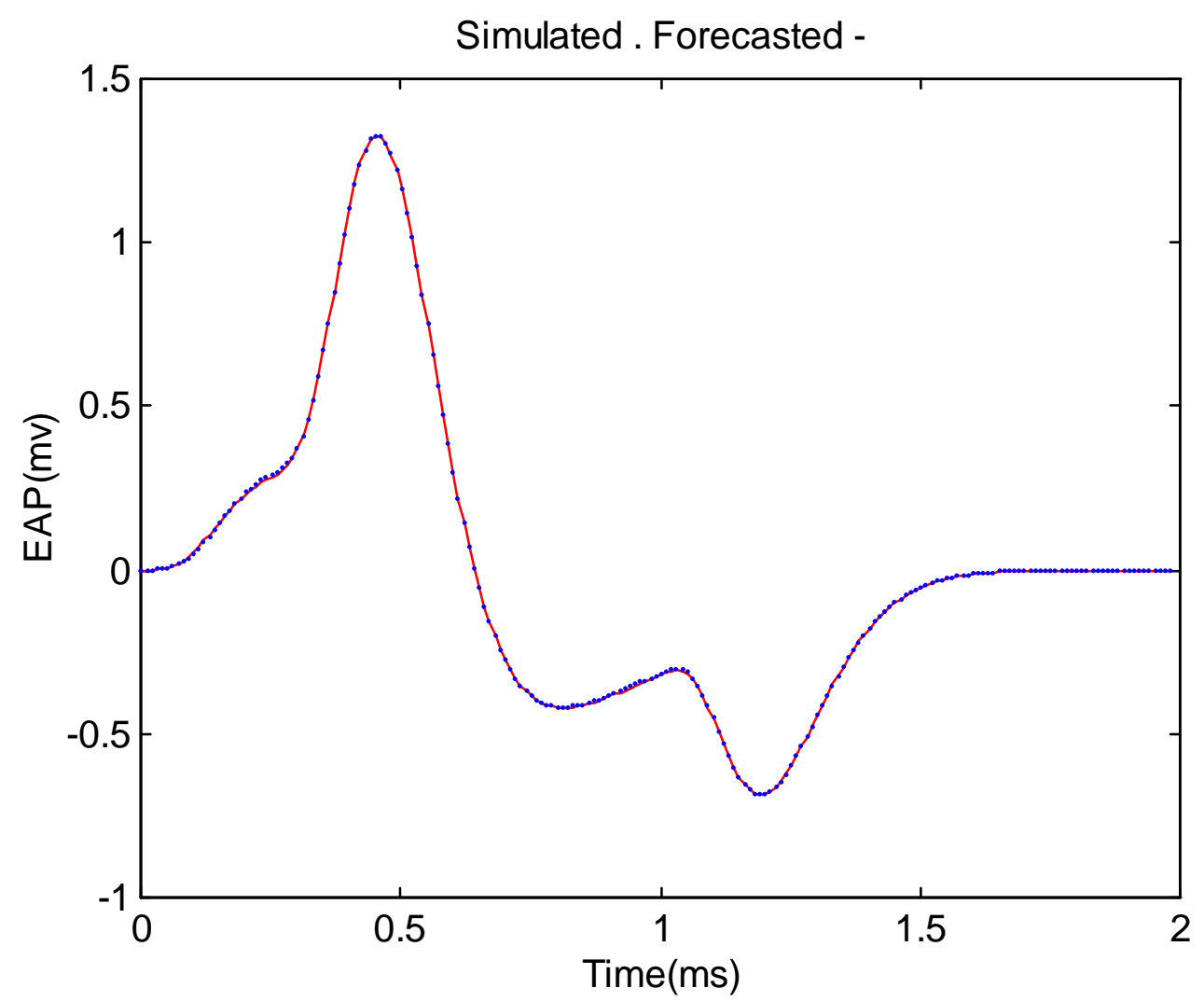

Fig. 4.18 Reinnervation of fibres. Simulation and decomposition of 75 fibres for a test radius of $5 \mathrm{~mm}$. Twenty five fibres were reinnervated in the right plane of the MU territory. No of iterations=1133. ( simulated blue '.', forecasted red '`') 
4.3.12 MUAP myopathy simulation 12, reinnervation of 25 fibres in the right plane of the MU territory: number of iterations 5295, Error=7.8821e-009.

Table A.4.19 in Appendix 4 gives the final values (results) for the $x$-horizontal distance, $y$-vertical distance, $z$-distance and $d$-diameter. It is shown that the error is almost infinitely small after 5295 iterations. Figure 4.19 illustrates the closeness between the simulated and final values as well as the overlap between the simulated and final waveform. 

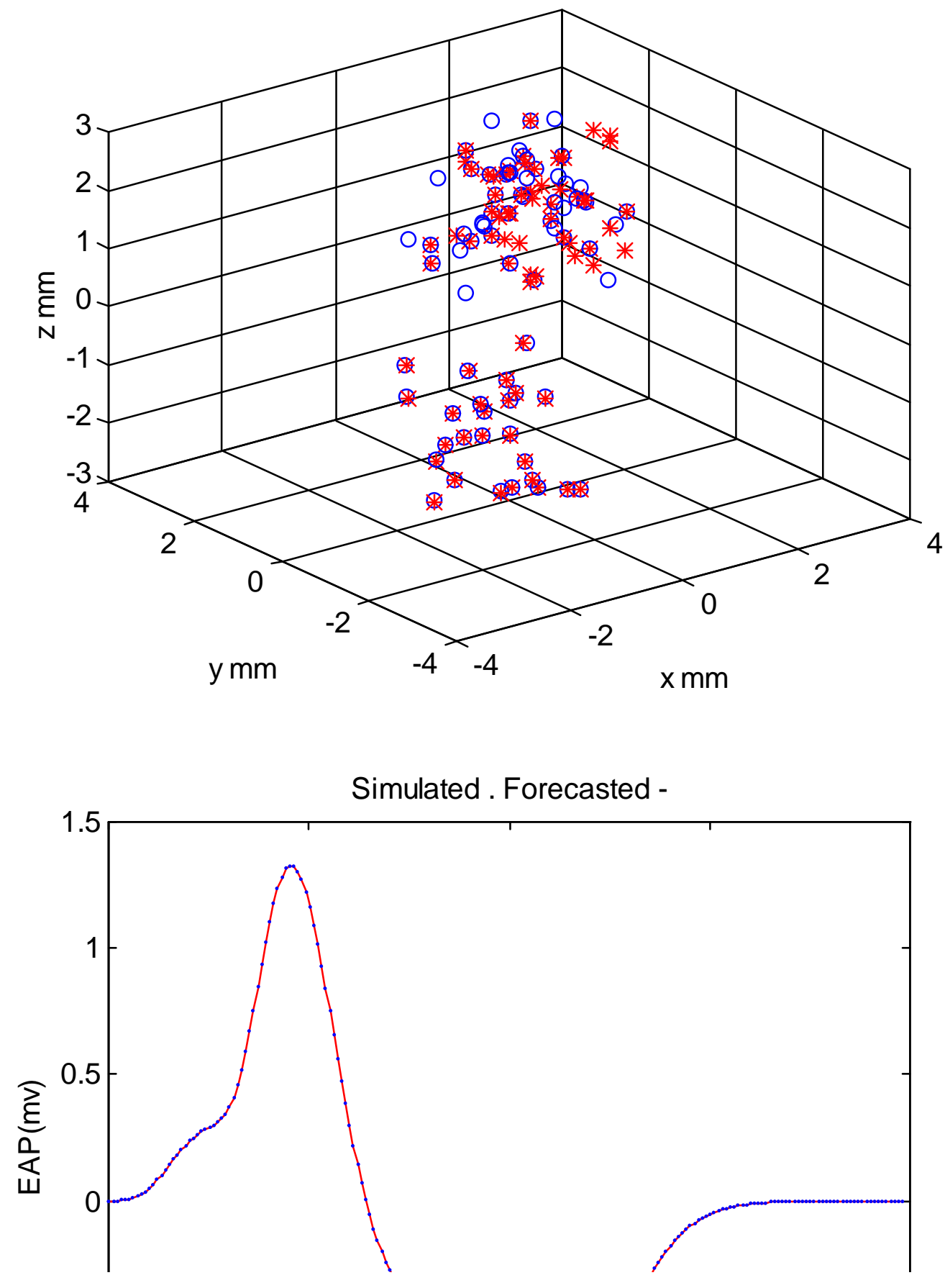

Fig. 4.19 Reinnervation of fibres. Simulation and decomposition of 75 fibres for a test radius of $5 \mathrm{~mm}$. Twenty five fibres were reinnervated in the right plane of the MU territory. No of iterations $=5295$. ( simulated blue '.', forecasted red '-'). The top graph shows the positioning of the fibres. (simulated blue 'o', forecasted red ' + ') 


\subsubsection{Summarized results for myopathy MUAP simulation using a three- dimensional model}

Table 4.7 shows the results for the simulation and decomposition of myopathic MUAPS. It is shown clearly that in all cases the error is infinitely small after approximately 5000 iterations.

\begin{tabular}{|c|c|c|c|c|c|c|}
\hline \multirow[t]{2}{*}{ Myopathy } & Simulated & Initial & Resulted & Test radius & No of & Error \\
\hline & Fibres & Fibres & Fibres & $(\mathrm{mm})$ & Iterations & \\
\hline \multicolumn{7}{|c|}{ I. Loss of fibres } \\
\hline & 25 & 25 & 25 & 5 & 221 & 0.3823 \\
\hline & 25 & 25 & 25 & 5 & 1115 & $2.0846 \mathrm{e}-005$ \\
\hline & 25 & 25 & 25 & 5 & 1113 & $3.8031 \mathrm{e}-007$ \\
\hline \multicolumn{7}{|c|}{ II. Variability in diameter } \\
\hline & 50 & 50 & 50 & 5 & 251 & 346.1319 \\
\hline & 50 & 50 & 50 & 5 & 1011 & 5.9174 \\
\hline & 50 & 50 & 50 & 5 & 5066 & $4.1195 \mathrm{e}-004$ \\
\hline \multicolumn{7}{|c|}{ III. Reinnervation of fibres. ( 25 fibres added in MU territory) } \\
\hline & 75 & 75 & 75 & 5 & 376 & 6.3581 \\
\hline & 75 & 75 & 75 & 5 & 1132 & 0.0087 \\
\hline & 75 & 75 & 75 & 5 & 5294 & $1.7908 \mathrm{e}-007$ \\
\hline \multicolumn{7}{|c|}{ IV. Reinnervation of fibres. (25 fibres added in the right plane of MU's territory) } \\
\hline & 75 & 75 & 75 & 5 & 376 & 3.4393 \\
\hline & 75 & 75 & 75 & 5 & 1133 & 0.0012 \\
\hline & 75 & 75 & 75 & 5 & 5295 & $7.8821 \mathrm{e}-009$ \\
\hline
\end{tabular}

Table 4.7 Summarized results for myopathic MUAPS using a three-dimensional model. 


\section{DISCUSSION}

The dipole model developed by Dimitrova 1974 and Dimitrov 1987 has been investigated for the problem of Model Based Decomposition of MUAPS into their Constituent SFEAPS. The non linear least squares optimization procedure based on the Levenberg-Marquardt algorithm has been used to obtain a solution to the MUAP decomposition problem i.e. fibre distribution and positioning and fibre diameter. Models with different complexity, representing normal and abnormal waveshapes have been generated and decomposed based on the proposed system. The system has been developed using the MATLAB program and consists of two main components, the simulation of MUAPS and the decomposition of the MUAPS into their constituent SFEAPS for normal and myopathy cases. The experiments and the results obtained are discussed below.

\subsection{MUAP Simulation and Decomposition Using a Two-Dimensional Model for Normal MUAPS}

MUAP simulations with 1, 4, 5, and 10 fibres within a test radius of $0.6 \mathrm{~mm}, 0.9 \mathrm{~mm}$, $1.0 \mathrm{~mm}$ and $1.2 \mathrm{~mm}$ respectively have been carried out using the dipole model developed by Dimitrova and Dimitrov (Dimitrova 1974; Dimitrov 1987). This model is described in Section 2.2.4. The non-linear least-squares algorithm LevenbergMarquardt algorithm, which is described in Section 3.3, was applied to achieve the decomposition of the simulated MUAPS into their constituent SFEAPS. The error was minimal $\left(4.7475 \mathrm{e}^{-11}, 1.9997 \mathrm{e}^{-13}, 6.3597 \mathrm{e}^{-017}, 9.0148 \mathrm{e}^{-16}\right)$ for the simulations of $1,4,5$ and 10 fibres after 17, 58, 53 and 97 iterations respectively and an overlap of the coordinates between the simulated and final values as well between the simulated and 
final waveforms has been achieved. The results are satisfactory and they are described in detail in Section 4.1.

\subsection{MUAP Simulation and Decomposition Using a Three-Dimensional Model for} Normal MUAPS.

MUAP simulations with 50 fibres within a test radius of $5 \mathrm{~mm}$ have been carried out. The same dipole model as in the case of two-dimensional models has been applied to achieve the simulations with the difference that a $3^{\text {rd }}$ coordinate has been added. Also the non-linear least-squares Levenberg-Marquardt algorithm has been applied to achieve the decomposition of the simulated MUAPS into their constituent SFEAPS. The algorithm has been repeated for 251, 1011 and 5065 iterations. The results after 5065 iterations were satisfactory as the error was minimal $\left(2.7241 \mathrm{e}^{-8}\right)$ and an overlap between the simulated and the final waveform has been achieved. The description of the results is given in detail in Section 4.2.

\subsection{MUAP Simulation and Decomposition Using a Three-Dimensional Model for Myopathy MUAPS}

Changes in the MU architecture affect the results of the MUAP simulations. MUAP simulations in cases of myopathy using a three-dimensional model have been carried out. The non-linear least-squares Levenberg-Marquardt algorithm again has been applied to achieve the decomposition of the simulated MUAPS. The following myopathy cases have been examined:

A. Loss of half of the fibres

A MUAP has been simulated with only 25 fibres out of 50 in the case of the normal MU territory. This is a pathological case where half fibres were considered lost. In this case, 
the error during the decomposition after 1113 iterations is infinitely small $\left(3.8031 \mathrm{e}^{-7}\right)$ and an overlap between the simulated and final waveform has been achieved. The description of the results is given in detail in Sections 4.3.1 - 4.3.3.

\section{B. $\quad$ Variability in the diameter}

A MUAP has been simulated and decomposed with variability in diameter (the diameter was twice as big as the diameter in normal cases). The algorithm was repeated for 251 , 1011 and 5066 iterations. The results after 5066 iterations were satisfactory as the error was minimal $\left(4.1195 \mathrm{e}^{-4}\right)$ and an overlap between the simulated and final waveform has been achieved. The description of the results is given in detail in Sections 4.3.4 - 4.3.6.

\section{Reinnervation of fibres}

Two cases of fibre reinnervation have been examined. In the first case, 25 fibres were added in both planes of the MU territory. In the second case 25 , fibres have been added in the right plane of MU territory. The same procedure has been followed as in the previous cases. In both cases, the error was minimal $\left(1.7908 \mathrm{e}^{-7}, 7.8821 \mathrm{e}^{-9}\right)$ after 5300 iterations approximately and an overlap between the simulated and final waveform has been achieved. The description of the results is given in detail in Sections 4.3.7 - 4.3.12. 


\section{CONCLUSIONS AND FUTURE WORK}

\subsection{Conclusions}

The MUAP represents the spatial and temporal summation of SFEAPS generated from the same motor unit. MUAP morphology is affected by structural reorganisation of the motor unit that takes place due to disorders affecting peripheral nerve and muscle. MUAP features extracted in the time domain like duration, amplitude and phases are extensively used by the neurophysiologist for the assessment of neuromuscular disorders. In this study, a model based decomposition of MUAPS into their constituent SFEAPS has been investigated.

Initially, the two-dimensional mathematical model developed by Dimitrova and Dimitrov (Dimitrova 1974; Dimitrov 1987) has been used to generate SFEAPS. The two dimensional model was then extended to a three-dimensional model. Typical MUAPS recorded from normal and myopathic muscle have been simulated. The non linear least squares optimization procedure based on Levenberg-Marquardt algorithm has been used to obtain a solution to the MUAP decomposition problem i.e. fibre distribution and positioning and fibre diameter. Two-dimensional and three-dimensional models for normal MUAPS consisting of 1, 4, 5, 10 and 50 fibres respectively were developed and decomposed for a small recoding radius up to $5 \mathrm{~mm}$. Also threedimensional models have been developed and decomposed for the myopathic cases (loss of fibres, variability in the diameter and reinnervation). In all the models investigated, satisfactory results have been obtained within a rather limited number of iterations.

These findings suggest that an "estimated" visualization of the structural organisation of the motor unit can be derived. This visualization gives to the neurophysiologist 
information about the number of fibres, fibre distribution and positioning and fibre diameter for the simulated cases investigated.

The main contributions of this study are:

a. The extension of the two-dimensional model developed by Dimitrova and Dimitrov to a three-dimensional model and;

b. The development of a new method to aid the neurophysiologist in gaining a better assessment of the muscle structural organisation.

Future work will investigate the usefulness of the proposed new method on MUAPS recorded from normal subjects and subjects suffering with neuromuscular disorders.

\subsection{Future Work}

In this study simulated MUAPS have been developed based on the mathematical dipole model and then decomposed into their constituent SFEAPS using the LevenbergMarquardt non linear least-squares optimisation algorithm. Although a limited number of MUAPS have been developed the results illustrate that the methodology followed is appropriate and promising. However, more work has to be carried out. Moreover, to the best of the Author's knowledge, this approach has not yet been investigated by other researches but only for the decomposition of surface EMG into SFEAPS and needle EMG into MUAPS. Possible directions of future research can be summarized as follows: 


\section{A. Decomposition of real EMG signals}

The methodology proposed in this study could be followed for the decomposition of MUAP signals recorded from both normal subjects as well as from subjects suffering with neuromuscular disorders. The MUAP signals should be recorded at slight voluntary contraction so that they can be easily identified and segmented. Moreover, the usefulness of the proposed methodology could be investigated for decomposing interference pattern EMG signals recorded at higher force. These recordings could be carried out using either needle or surface electrodes. It is well understood that trying the proposed methodology in the cases of signals recorded at higher force with both needle and/or surface electrodes is a very difficult task, however it is worth of investigating. This investigation could be carried out in collaboration with the Department of Clinical Neurophysiology of the Cyprus Institute of Neurology and Genetics, with which our team is strongly collaborating.

\section{B. Decomposition of signals using other optimisation algorithms}

The use of other optimisation algorithms based on evolutionary computation (Fogel 1995, Michalewicz 1995) could also be investigated. Most importantly a hybrid optimisation methodology can be followed, where the evolutionary algorithm is used to compute an initial solution to the optimisation problem, and then, the classical leastsquares optimisation technique can be used to derive the final solution. This approach has already been used successfully in other optimisation problems, including optimal bandwidth allocation in communication links (Pitsillides et. al. 2002). 


\section{C. $\quad$ Simulation of MUAPS using more complicated SFEAP models}

SFEAP decomposition based on more complicated models could also be developed. These models could take into account the spatial coordinates, and the fibre diameter (as was the case of this study), as well as the delay information between the signal generators and the recording electrode. In addition, the firing statistics of the SFEAP signals can also be taken into consideration.

\section{Training Software}

The methodology followed in this study could be easily expanded with additional functionality and a user friendly interface that can be used for the simulation of MUAPS under different disease processes. The system could be used for training new neuroscientists.

It is hoped that this study will help in the understanding of muscle structure and pathophysiology, contributing towards the development of new techniques, aiding the neurophysiologist in gaining a better assessment of the muscle structural organisation. This could be eventually lead to the offering of a better service to the citizen. 


\section{REFERENCES}

Aquilonius S, Askmark H, Gilberg P, Nandedkar S, Olsson Y, Stalberg E, Topographical localization of motor endplates in cryosections of whole human muscles. Muscle \& Nerve, 7: 287-293, 1984.

Bertorini TE, Stalberg E, Yuson CP, Engel WK, Single fibre electromyography in neuromuscular disorders: correlation of muscle histochemistry, single fibre electromyography, and clinical findings, Muscle Nerve, 17(3): 345-53, 1994.

Bromberg MB, Scott DM, Single fibre EMG reference values: reformatted in tabular form. AD HOC Committee of the AAEM Single Fibre Special Interest Group, Muscle Nerve, 17(7): 820-1, 1994.

Buchthal F, Kamieniecka Z, The diagnostic yield of quantified electromyography and quantified muscle biopsy in neuromuscular disorders. Muscle \& Nerve 1982; 5:265280.

Clark J, Plonsey R, A mathematical evaluation of the core conductor model, Biophys J, 6(1): 95-112, 1966.

Clark J, Plonsey R, The extracellular potential field of the single active nerve fibre in a volume conductor, Biophys J 8(7): 842-64, 1968.

Clark JW Jr, Plonsey R, Fibre interaction in a nerve trunk, Biophys J, 11(3): 281-94, 
1971.

Clark JW, Plonsey R, A mathematical study of nerve fibre interaction, Biophys J, 10(10): 937-57, 1970.

Dimitrov GV, Changes in the extracellular potentials produced by unmyelinated nerve fibre resulting from alterations in the propagation velocity of the duration of the action potential. Electromyogr. Clin. Neurophysiol. 27, pp. 243-249, 1987.

Dimitrov GV, Lateva ZC, Dimitrova NA, Model of the slow components of skeletal muscle potentials. Med. \& Biol. Eng. \& Comput., 32, pp. 432-436, 1994.

Dimitrova NA, Model of the extracellular potential field of a single striated muscle fibre. Electromyogr. Clin. Neurophysiol., 14, pp. 53-66, 1974.

Dubowitz V, Brooke M, Muscle Biopsy: A Modern Approach. Philadelphia, W.B. Saunders, 1973.

Fogel DB. Evolutionary Computation, IEEE Press, NJ, USA, 1995.

Gill, PR, Murray, W. and Wright, MH: The Levenberg-Marquardt Method. §4.7.3 in Practical Optimization, Academic Press, London 1981, 136-137.

Graupe D, Vern B, Gruener G, Field and Huang Q, Decomposition of Surface EMG Signals into Single Fiber Action Potentials by Means of Neural Networks, IEEE Intern. Symposium on Circuits and Systems 10: 1008-1011, 1988. 
Greco EC, Clark JW, Harman TL, Volume - Conductor Fields of the Isolated Axon, Mathematical Biosciences 33:235-256, 1977.

Gydikov A, Kosarov D, Volume conduction of the potentials from separate motor units in human muscle, Electromyogr-Clin-Neurophysiol, 12(2): 127-47, 1972.

Henneberg K, Plonsey R, Spatial and frequency domain ring source models for the single muscle fibre action potential, Med Biol Eng Comput, 32(1): 27-34, 1994.

Henneberg KA, Plonsey R, Boundary element analysis of the directional sensitivity of the concentric EMG electrode, IEEE Trans Biomed Eng, 40(17): 621-31, 1993.

Jabre JF, Concentric macro electromyography, Muscle Nerve, 14(9): 820-5, 1991.

Larsson LE, On the relation between the EMG frequency spectrum and the duration of symptoms in lesions of the peripheral motor neuron, Electroencephalogr. Clin. Neurophysiol. Vol. $38: 69-79,1975$.

LeFever RS and De Luca CJ, Procedure for Decomposing the Myoelectric Signal Into Its Constituent Action Potentials - Part I: Technique, Theory, and Implementation. IEEE Transactions on Biomedical Engineering, Vol. BME-29, NO. 3: 149-157, March 1982. 
LeFever RS, Xenakis AP and De Luca CJ, Procedure for Decomposing the Myoelectric Signal Into Its Constituent Action Potentials - Part II: Execution and Test for Accuracy, IEEE Transactions on Biomedical Engineering, Vol. BME-29, NO. 3: 158-164, March 1982.

McGill KC and Dorfman LJ, Automatic Decomposition Electromyography (ADEMG): Validation and Normadive Data in Brachial Biceps, Electromyography and clinical Neurology, 61:453-461, 1985.

Michalewicz Z, Genetic Algorithm+Data Structures=Evolution Programs. Third, Revised and Extended Edition. Springer, Berlin, Germany, 1995.

More JJ, The Levenberg-Marquardt algorithm: implementation and theory. In Numerical Analysis, ed. G.A. Watson, Lecture Notes in Mathematics 630, SpringerVerlag, pp. 105-116, 1977.

Nakanishi T, Test for analysing nerve conduction velocity, Rinsho Shinkeigaku, 31(12): 1326-9, 1991.

Nandedkar SD, Models and Simulations in Electromyography, Muscle \& Nerve Supplement 11 : S46-S54,2002

Nandedkar SD, Sanders DB and Stalberg EV, EMG of reinnervated motor units: a simulation study, Electromyography and clinical Neurophysiology, 70: 177-184, 1988. 
Oostendorp $\mathrm{T}$, van-Oosterom $\mathrm{A}$, The potential distribution generated by surface electrodes in inhomogeneous volume conductors of arbitrary shape, IEEE Trans Biomed Eng, 38(5): 409-17, 1991.

C.S. Pattichis, A. Elias, Autoregressive and Cepstral Analysis of Motor Unit Action Potentials, Medical Engineering \& Physics, Special Issue on Intelligent Data Analysis in Electromyography and Electroneurography, Vol. 21, No. 6/7, pp. 405-419, 1999.

Pattichis CS and Pattichis MS, Time-Scale Analysis of Motor Unit Action Potentials, IEEE Transactions on Biomedical Engineering, Vol. 46, NO. 11:1320-1329, November 1999.

Pattichis CS, and Schizas CN, Genetics-Based Machine Learning Models for the Diagnosis of Certain Neuromuscular Disorders, IEEE Transactions on Neural Networks, Vol. 7, No. 2, pp. 427-439, 1996.

Pattichis CS, Pattichis MS, Time-Scale Analysis of Motor Unit Action Potentials, IEEE Transactions on Biomedical Engineering, Vol. 46, No. 11, pp. 1320-1329, 1999.

Pattichis CS, Schizas CN, Middleton L, Neural Network Models in Classifying Motor Unit Potential Parameters, IEEE Transactions on Biomedical Engineering, Vol. 42, No. 5, pp. 486-496, 1995.

Pattichis CS, Schofield I,Merletti R, Parker PA, Middleton LT, Introduction to this Special Issue: Intelligent Data Analysis on Electromyography and Electroneurography, 
Medical Engineering and Physics Vol. 21, No. 6/7, pp. 379-388, 1999.

Pitsillides A, Stylianou G, Pattichis CS, Sekercioglu A, Vassilakos T, Bandwidth Allocation for Virtual Paths (BAVP): Investigation of Performance of Classical Constrained and Genetic Algorithm Based Optimisation Techniques, Journal on Computer Communications, Special Issue On Computational Intelligence In Telecommunications Networks, Vol. 25, No. 16, pp. 1443-1453, 2002.

Plonsey R, Heppner DB, Considerations of quasi-stationarity in electrophysiological systems, 29(4): 657-64, 1967.

Plonsey R, On volume conductor fields of compound nerve action potentials, Med Biol Eng, 7(2): 253-4, 1969.

Quantitative analysis of individual motor unit potentials: A proposition for standardized terminology and criteria for measurement. J. Of Clin. Neurophysiol., 3(4):313-348, Raven Press, New York, 1986.

Rosenfalck A, Choice of electrode, amplifier and auxiliary equipment for the recording of muscle and nerve action potentials in man, Electroencephalogr-Clin-Neurophysiol, 25(4): 400, 1968.

Rosenfalck P, Intra-and extracellular potential fields of active nerve and muscle fibres. A physioco-mathematical analysis of different models, Throb-Diath-Haemorrh-Suppl, 321: 1-168, 1969. 
Ruurd Schoonoven, Dick F. Stegeman, Adriaan Van Oosterom and Guido F. M. Dautzenberg, The Inverse Problem in Electromyography -I: Conceptual Basis and Mathematical Formulation, IEEE Transactions on Biomedical Engineering, Vol. 35, NO. 10:769-776, October 1988.

Seagar AD, Grognard RJ, Simulation of current flow in piecewise constant media, Australas Phys Eng Sci Med, 14(4): 204-12, 1991.

Stalberg E, Andreassen S, Falck B, Lang H, Rosenfalck A, Trojaborg W, Quantitave analysis of individual motor unit potentials: A proposition for standardized terminology and criteria for measurement. J. Of Clin. Neurophysiol. 3(4):313-348, Raven Press, New York, 1986.

Stegeman DF, Gootzen TH, Theeuwen MM, Vingerhoets HJ, Intramuscular potential changes caused by the presence of the recording EMG needle electrode, Electroencephalogr Clin Neurophysiol, 93(2): 81-90, 1994.

Takeda K, Tojima H, Aoyagi M, Koike Y, Recording of single fibre electromyography in patients with peripheral facial palsy, Acta Otolaryngol Suppl Stockh, 511: 156-60, 1994.

Thevenet M, Bertrand O, Perrin F, Dumont T, Pernier J, The finite element method for a realistic head model of electrical brain activities: preliminary results, Clin Phys Physiol Meas, 12 suppl A: 89-94, 1991. 


\section{APPENDIX 1}

\section{MATLAB FUNCTIONS FOR TWO-DIMENSIONAL MODELS}

\section{A.1.1 FUNCTION INVMUAP}

function invmuap=invmuap(z);

$\%$ columns of matrix

$\% 11,12,131415-\mathrm{x}$

$\% 21,22,23,24,25-\mathrm{y}$

$\% 31,32,33,34,35-\mathrm{d}$

$\% \mathrm{nr}=$ real number of fibres constint

$\% \mathrm{nc}=$ calculated number of fibres

$\mathrm{v}=4 ; \mathrm{kan}=5 ; \mathrm{vm}=1 ; \mathrm{si}=1.01 ; \mathrm{sx}=0.33 ; \mathrm{sy}=0.63$;

$\mathrm{nc}=1$;

pcalc $=0$;

$\% 1$ fibre $\mathrm{r}=\left[\begin{array}{llll}0.3750 & 0.3946 & 0.0328\end{array}\right]$;

$\% 4$ fibre

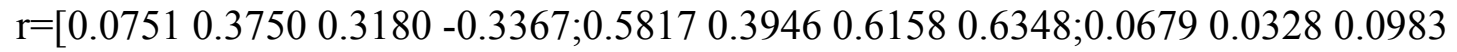
$0.0478]$

$\%$ not used 3 fibres $r=\left[\begin{array}{llllll}0.0644 & -0.4002 & 0.2843 ; 0.0083 & 0.1571 & 0.3826 ; 0.0899 & 0.0700\end{array}\right.$

$0.0651]$

$\% 5$ fibres

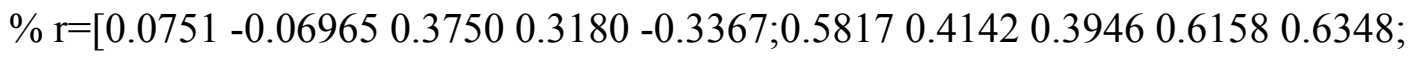

$0.06790 .09350 .03280 .09830 .0478]$;

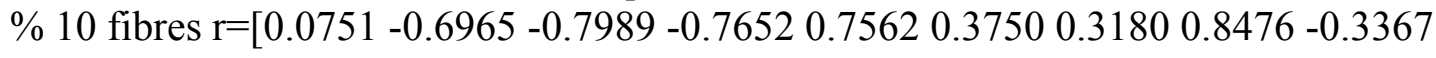

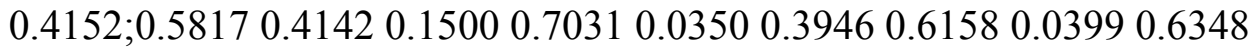

$\left.\begin{array}{lllllllllll}0.8204 ; 0.0679 & 0.0935 & 0.0092 & 0.0654 & 0.0262 & 0.0328 & 0.0983 & 0.073 & 0.0478 & 0.0238\end{array}\right]$

p_real1=tot_out3 $\left(\mathrm{v}, \mathrm{r}(1,1)^{*} 10^{\wedge}(-3), \mathrm{r}(2,1)^{*} 10^{\wedge}(-3), \mathrm{r}(3,1)^{*} 10^{\wedge}(-3), \mathrm{kan}, \mathrm{vm}, \mathrm{si}, \mathrm{sx}, \mathrm{sy}\right)$;

p_calc1=tot_out $3\left(\mathrm{v}, \mathrm{z}(1,1)^{*} 10^{\wedge}(-3), \mathrm{z}(2,1)^{*} 10^{\wedge}(-3), \mathrm{z}(3,1)^{*} 10^{\wedge}(-3), \mathrm{kan}, \mathrm{vm}, \mathrm{si}, \mathrm{sx}, \mathrm{sy}\right)$;

p_real2=tot_out3 $\left(\mathrm{v}, \mathrm{r}(1,2)^{*} 10^{\wedge}(-3), \mathrm{r}(2,2)^{*} 10^{\wedge}(-3), \mathrm{r}(3,2)^{*} 10^{\wedge}(-3), \mathrm{kan}, \mathrm{vm}, \mathrm{si}, \mathrm{sx}, \mathrm{sy}\right)$; p_calc2=tot_out3 $\left(\mathrm{v}, \mathrm{z}(1,2)^{*} 10^{\wedge}(-3), \mathrm{z}(2,2)^{*} 10^{\wedge}(-3), \mathrm{z}(3,2)^{*} 10^{\wedge}(-3), \mathrm{kan}, \mathrm{vm}, \mathrm{si}, \mathrm{sx}, \mathrm{sy}\right)$;

p_real3=tot_out3 $\left(\mathrm{v}, \mathrm{r}(1,3) * 10^{\wedge}(-3), \mathrm{r}(2,3)^{*} 10^{\wedge}(-3), \mathrm{r}(3,3) * 10^{\wedge}(-3), \mathrm{kan}, \mathrm{vm}, \mathrm{si}, \mathrm{sx}, \mathrm{sy}\right)$; p_calc3=tot_out3 $\left(\mathrm{v}, \mathrm{z}(1,3) * 10^{\wedge}(-3), \mathrm{z}(2,3) * 10^{\wedge}(-3), \mathrm{z}(3,3) * 10^{\wedge}(-3), \mathrm{kan}, \mathrm{vm}, \mathrm{si}, \mathrm{sx}, \mathrm{sy}\right)$;

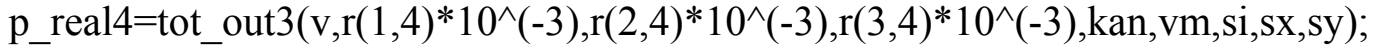

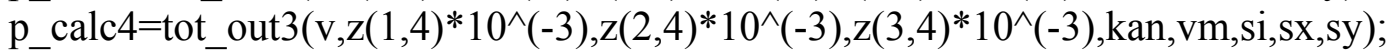

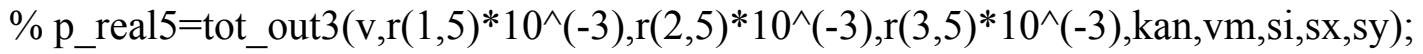

$\%$ p_calc5=tot_out3 $\left(\mathrm{v}, \mathrm{z}(1,5) * 10^{\wedge}(-3), \mathrm{z}(2,5) * 10^{\wedge}(-3), \mathrm{z}(3,5) * 10^{\wedge}(-3), \mathrm{kan}, \mathrm{vm}, \mathrm{si}, \mathrm{sx}, \mathrm{sy}\right)$;

$\%$ p_real6=tot_out3 $\left(\mathrm{v}, \mathrm{r}(1,6)^{*} 10^{\wedge}(-3), \mathrm{r}(2,6) * 10^{\wedge}(-3), \mathrm{r}(3,6)^{*} 10^{\wedge}(-3), \mathrm{kan}, \mathrm{vm}, \mathrm{si}, \mathrm{sx}, \mathrm{sy}\right)$;

$\%$ p_calc6 $=$ tot_out3(v,z $\left.(1,6) * 10^{\wedge}(-3), \mathrm{z}(2,6) * 10^{\wedge}(-3), \mathrm{z}(3,6) * 10^{\wedge}(-3), \mathrm{kan}, \mathrm{vm}, \mathrm{si}, \mathrm{sx}, \mathrm{sy}\right)$;

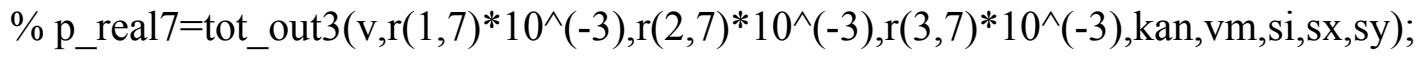


$\%$ p_calc7 $=$ tot_out $3\left(\mathrm{v}, \mathrm{z}(1,7) * 10^{\wedge}(-3), \mathrm{z}(2,7)^{*} 10^{\wedge}(-3), \mathrm{z}(3,7) * 10^{\wedge}(-3), \mathrm{kan}, \mathrm{vm}, \mathrm{si}, \mathrm{sx}, \mathrm{sy}\right)$;

$\%$ p_real8=tot_out3(v,r $\left.(1,8)^{*} 10^{\wedge}(-3), \mathrm{r}(2,8)^{*} 10^{\wedge}(-3), \mathrm{r}(3,8)^{*} 10^{\wedge}(-3), \mathrm{kan}, \mathrm{vm}, \mathrm{si}, \mathrm{sx}, \mathrm{sy}\right)$; $\%$ p_calc8=tot_out3 $\left(\mathrm{v}, \mathrm{z}(1,8) * 10^{\wedge}(-3), \mathrm{z}(2,8)^{*} 10^{\wedge}(-3), \mathrm{z}(3,8) * 10^{\wedge}(-3), \mathrm{kan}, \mathrm{vm}, \mathrm{si}, \mathrm{sx}, \mathrm{sy}\right)$;

$\%$ p_real9=tot_out3 $\left(\mathrm{v}, \mathrm{r}(1,9)^{*} 10^{\wedge}(-3), \mathrm{r}(2,9)^{*} 10^{\wedge}(-3), \mathrm{r}(3,9)^{*} 10^{\wedge}(-3), \mathrm{kan}, \mathrm{vm}, \mathrm{si}, \mathrm{sx}, \mathrm{sy}\right)$; $\% \mathrm{p} \_$calc9=tot_out3 $\left(\mathrm{v}, \mathrm{z}(1,9)^{*} 10^{\wedge}(-3), \mathrm{z}(2,9) * 10^{\wedge}(-3), \mathrm{z}(3,9)^{*} 10^{\wedge}(-3), \mathrm{kan}, \mathrm{vm}, \mathrm{si}, \mathrm{sx}, \mathrm{sy}\right)$;

$\%$ p_real10 $=$ tot_out3 $\left(\mathrm{v}, \mathrm{r}(1,10) * 10^{\wedge}(-3), \mathrm{r}(2,10) * 10^{\wedge}(-3), \mathrm{r}(3,10) * 10^{\wedge}(-\right.$

3),kan,vm,si,sx,sy);

$\% \mathrm{p} \_$calc10 $=$tot_out $3\left(\mathrm{v}, \mathrm{z}(1,10) * 10^{\wedge}(-3), \mathrm{z}(2,10) * 10^{\wedge}(-3), \mathrm{z}(3,10) * 10^{\wedge}(-\right.$

3),kan,vm,si,sx,sy);

p_rtot $=p \_r e a 11+p \_r e a 12+p \_r e a 13+p \_r e a l 4 ;$

$\mathrm{p} \_$ctot $=\mathrm{p} \_$calc $1+\mathrm{p} \_$calc $2+\mathrm{p} \_$calc $3+\mathrm{p} \_$calc 4 ;

invmuap $=$ p_rtot-p_ctot;

\section{A.1.2 FUNCTION TOT_OUT3}

function tot_out3=tot_out3(v,x0,y0,d,kan,vm,si,sx,sy)

total $=0$;

for $\mathrm{t}=0: 6.2^{*} 10^{\wedge}(-2): 6.2^{*} 10^{\wedge}(-1)$,

$\mathrm{tx}=\left(0: 10^{\wedge}(-2): \mathrm{t}\right)$;

$\mathrm{k} 1=$ out3 $(\mathrm{kan}, \mathrm{v}, \mathrm{tx}, \mathrm{x} 0, \mathrm{y} 0)$;

total $=[\mathrm{k} 1]$;

end

$\mathrm{ce}=\mathrm{c} 1(\mathrm{~d}, \mathrm{kan}, \mathrm{vm}, \mathrm{si}, \mathrm{sx}, \mathrm{sy})$;

tot_out $3=-$ ce*total;

\section{A.1.3 FUNCTION OUT3}

function out $3=$ out $3(\mathrm{kan}, \mathrm{v}, \mathrm{tx}, \mathrm{x} 0, \mathrm{y} 0)$

$\%$ fx $1={ }^{\prime} 72234 * x^{\wedge} 5 * \exp (-11 * x)^{\prime}$

$\%$ diff_fx $1=(\exp (-11 * x)) *\left(361170 * x^{\wedge} 4-794574 * x^{\wedge} 5\right)$

$\left.\% \mathrm{wfl}=-1 /\left(\mathrm{x}^{\wedge} 2+\mathrm{y} 0^{\wedge} 2\right)\right)^{\wedge}(3 / 2)^{*} \mathrm{x}$;

$\mathrm{x}=\mathrm{v} * \mathrm{tx}$;

$\operatorname{diff} 1=\exp (-11 * x)$;

$\operatorname{diff} 2=361170 * x .^{\wedge} 4-794574 * x .^{\wedge} 5$;

diff_fx $1=\operatorname{diff} 1 *^{*} \operatorname{diff} 2$;

$\%$ wf1 $1=\mathrm{x} .{ }^{\wedge} 2+5^{*} \mathrm{y} 0 \wedge 2$;

$\%$ wf11=wf1 1.^(3/2);

$\% \mathrm{wfl}=-\mathrm{wfl1} .^{*} \mathrm{x}$;

$\mathrm{wf1}=$ diff_ran $(\mathrm{kan}, \mathrm{x} 0, \mathrm{y} 0,0, \mathrm{x})$;

out3=conv(diff_fx1,wf1); 


\section{A.1.4 FUNCTION DIFF_RUN}

function diff_ran=diff_ran $(\mathrm{kan}, \mathrm{x} 0, \mathrm{y} 0, \mathrm{y}, \mathrm{x})$

$\%$ Rosenfalk Value: kan $=5$

$\%$ ran $=^{\prime}\left((\mathrm{x}-\mathrm{x} 0)^{\wedge} 2+\mathrm{kan} *(\mathrm{y}-\mathrm{y} 0)^{\wedge} 2\right)^{\wedge} 1 / 2^{\prime}$

$\%$ inv_ran='(x-x0)^2+kan* $\left.(\mathrm{y}-\mathrm{y} 0)^{\wedge} 2\right)^{\wedge}(-1 / 2)^{\prime}$

$\%$ diff_ran=diff(inv_ran,' $x$ ')

$\%$ diff_ran $=-1 / 2 /\left((\mathrm{x}-\mathrm{x} 0)^{\wedge} 2+\mathrm{kan} *(\mathrm{y}-\mathrm{y} 0)^{\wedge} 2\right)^{\wedge}(3 / 2)^{*}(2 * \mathrm{x}-2 * \mathrm{x} 0)$

diff_ran $=(\mathrm{x}-\mathrm{x} 0) . \wedge 2$;

diff_ran=diff_ran $+\mathrm{kan} *(\mathrm{y}-\mathrm{y} 0)^{\wedge} 2$;

diff_ran=diff_ran.^(3/2);

diff_ran $=-1 / 2 *$ diff_ran.^(-1);

diff_ran=diff_ran. $*(2 * x-2 * x 0)$;

\section{A.1.5 FUNCTION COMMUAPN}

function commuapn=commuapn(rn,on, count,counto);

$\%$ count - real number of fibers

$\%$ counto - calculated number of fibers

$\mathrm{v}=4 ; \mathrm{d} 1=45^{*} 10^{\wedge}(-6) ; \mathrm{kan}=5 ; \mathrm{vm}=1 ; \mathrm{si}=1.01 ; \mathrm{sx}=0.33 ; \mathrm{sy}=0.63 ; \mathrm{n}=1 ;$ pers $=0$;

$\mathrm{d} 2=55^{*} 10^{\wedge}(-6) ; \mathrm{d} 3=50^{*} 10^{\wedge}(-6) ; \mathrm{d} 4=48^{*} 10^{\wedge}(-6) ; \mathrm{d} 5=49^{*} 10^{\wedge}(-6)$;

if count $==1$ ptitle $=$ 'A. Test radius $=0.6 \mathrm{~mm}$ No of fibres $=1$;

end

if count $==4$ ptitle $=$ 'B. Test radius $=0.9 \mathrm{~mm}$ No of fibres $=4$;

end

if count $==5$ ptitle $={ }^{\prime} C$. Test radius $=1.0 \mathrm{~mm}$ No of fibres $=5^{\prime}$;

end

if count $==10$ ptitle $=$ 'D. Test radius $=1.2 \mathrm{~mm}$ No of fibres $=10$ ';

end

muap_real $=0$;

muap_real_anat=[];

muap_out $=0$;

muap_out_anat $=[]$;

$\mathrm{rx}=[]$

ry $=[]$

$\mathrm{rd}=[]$

$\mathrm{ox}=[]$;

oy=[];

$\mathrm{od}=[]$;

$\mathrm{r}=\mathrm{rn}$;

$\mathrm{o}=\mathrm{on}$;

$\% * * * * * * * * * * * *$ real values 


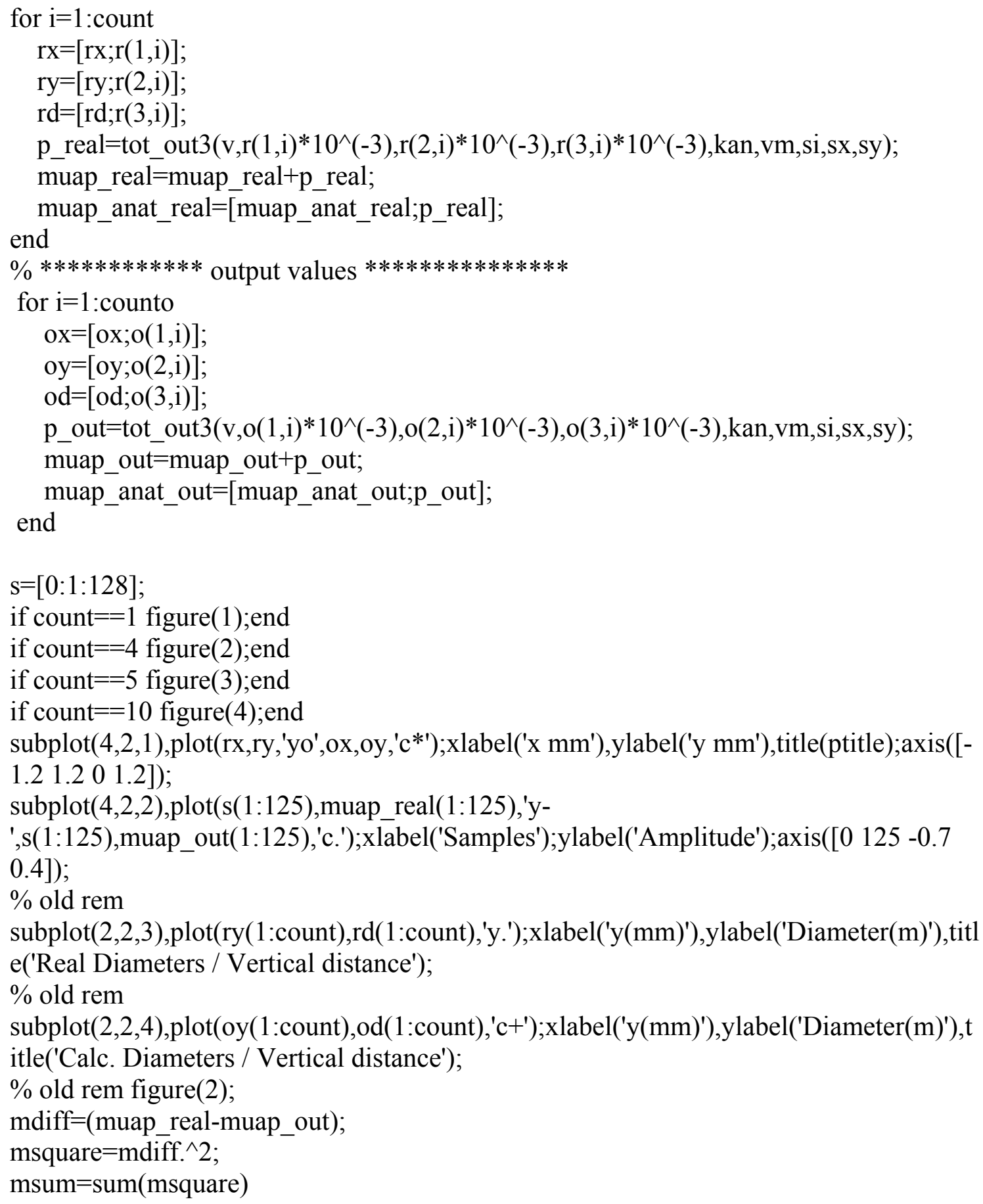




\section{APPENDIX 2}

\section{MATLAB FUNCTIONS FOR THREE-DIMENSIONAL MODELS}

\section{A.2.1 FUNCTION SIMU0208}

function simu $0208=$ simu 0208 ;

$\mathrm{n}=50 ; \%$ no of fibers

$\% \mathrm{p}=\operatorname{rand}(5,50)$;

load real_p; \%equal with rand $(5,50)$

$\mathrm{p}=$ real $\mathrm{p}$;

$\% \quad$ p11,p12 .... x 1 - x coordinate of end plates

$\% \quad$ p21,p22 .... y1 - y coordinate of end plates

$\% \quad$ p31,p32 .... z1 - z coordinate of end plates

$\% \quad$ p41,p42 .... diameter of each fiber

$\% \quad$ p51,p52 .... delay of eap of each fiber

$\mathrm{p}$

for $\mathrm{i}=1: 5$,

for $\mathrm{j}=1: 50$,

if $i==1$

$p(i, j)=p(i, j) * 2.5$;

elseif $i==2$

$p(i, j)=p(i, j) * 2.5$;

elseif $i==3$

$p(i, j)=p(i, j) * 2.5$;

elseif $i==4$

$$
p(i, j)=(\operatorname{abs}(p(i, j) * 12-6)+50)^{*} 10^{\wedge}(-2) \text {; }
$$

else

$p(i, j)=a b s(p(i, j)) * 10$;

end;

end

end

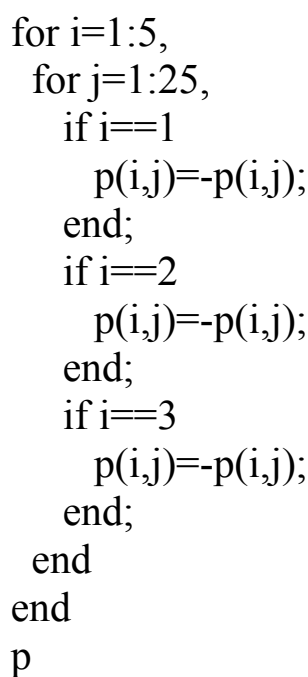




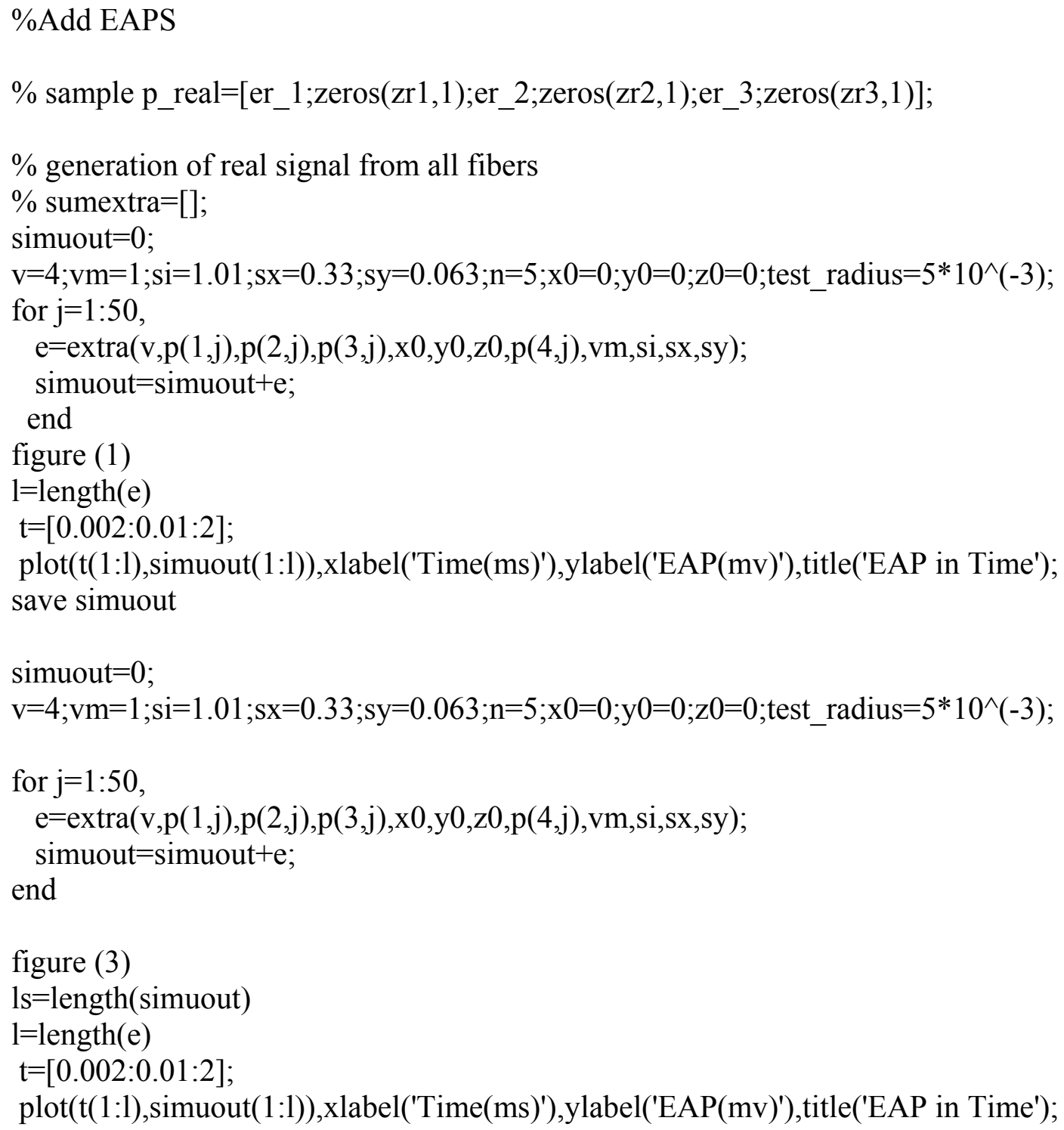

\section{A.2.2 FUNCTION NORMAL2}

function normal2 $=$ normal2 $(\mathrm{z})$;

$\%$ this function is used to simulate normal muap and then decompose

\%load final_z to be used as $\mathrm{z}$

\section{\%Add EAPS}

$\%$ generation of the calculated signal from all fibers using $\mathrm{z}$

calextra $=0$;

$\mathrm{v}=4 ; \mathrm{vm}=1 ; \mathrm{si}=1.01 ; \mathrm{sx}=0.33 ; \mathrm{sy}=0.063 ; \mathrm{n}=5 ; \mathrm{x} 0=0 ; \mathrm{y} 0=0 ; \mathrm{z} 0=0 ;$ test_radius $=5 * 10^{\wedge}(-3)$; for $\mathrm{j}=1: 50$, $\mathrm{e}=\operatorname{extra}(\mathrm{v}, \mathrm{z}(1, \mathrm{j}), \mathrm{z}(2, \mathrm{j}), \mathrm{z}(3, \mathrm{j}), \mathrm{x} 0, \mathrm{y} 0, \mathrm{z} 0, \mathrm{z}(4, \mathrm{j}), \mathrm{vm}, \mathrm{si}, \mathrm{sx}, \mathrm{sy})$; calextra $=$ calextra + ; 
end

$\%$ keep z

temp_z=z;

$\% \% \% \% \% \% \% \% \%$ known signal $\% \% \% \% \% \% \% \% \% \% \% \% \% \% \% \% \% \% \% \% \% \% \% \% \%$ load real_p; \%50 fibers

$\mathrm{p}=$ real_p;

for $\mathrm{i}=1: 5$,

for $\mathrm{j}=1: 50$,

if $i==1$

$p(i, j)=p(i, j) * 2.5$;

elseif $i==2$

$p(i, j)=p(i, j) * 2.5$;

elseif $i==3$

$p(i, j)=p(i, j) * 2.5$;

elseif $i==4$

$p(i, j)=(\operatorname{abs}(p(i, j) * 12-6)+50)^{*} 10^{\wedge}(-2)$;

else

$p(i, j)=a b s(p(i, j)) * 10 ;$

end;

end

end

$\%$ half fibers are positioned before zero $(\mathrm{x}, \mathrm{y}, \mathrm{z})$

for $i=1: 5$,

for $\mathrm{j}=1: 50 / 2$,

if $i==1$

$p(i, j)=-p(i, j) ;$

end;

if $i==2$

$p(i, j)=-p(i, j)$;

end;

if $i==3$

$p(i, j)=-p(i, j)$;

end;

end

end

$\%$ p_modif $=\mathrm{p}$;

\%save p_modif;

$\%$ retrieve $\mathrm{z}$

$\mathrm{z}=$ temp_z;

$\%$ generation of real signal from all fibers 


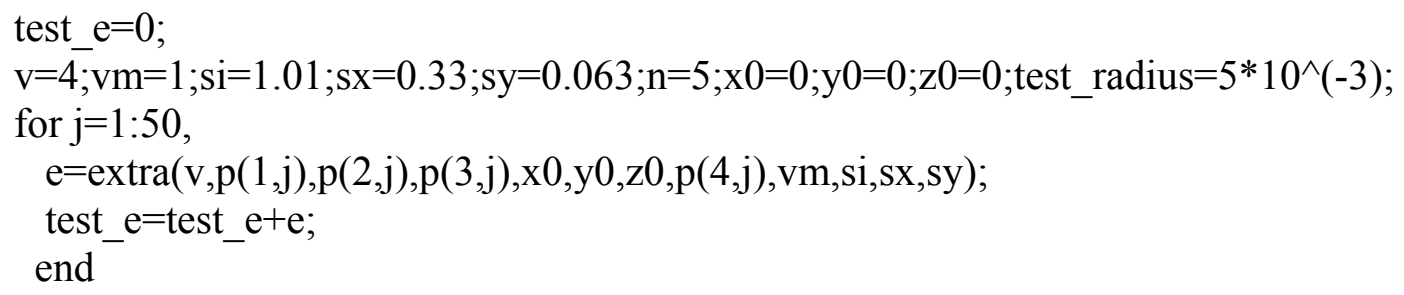

\section{A.2.3 FUNCTION HALFLOSS}

function halfloss $=$ halfloss $(\mathrm{z})$;

$\%$ this function is used to simulate myopathic muap (half fibres are lost) and then decompose

\%load zhalf to be used as $\mathrm{Z}$

\section{$\%$ Add EAPS}

$\%$ generation of the calculated signal from 25 fibers using $\mathrm{z}$

calextra $=0$

$\mathrm{v}=4 ; \mathrm{vm}=1 ; \mathrm{si}=1.01 ; \mathrm{sx}=0.33 ; \mathrm{sy}=0.063 ; \mathrm{n}=5 ; \mathrm{x} 0=0 ; \mathrm{y} 0=0 ; \mathrm{z} 0=0 ;$ test_radius $=5^{*} 10^{\wedge}(-3)$; for $\mathrm{j}=20: 44$, $\mathrm{e}=\operatorname{extra}(\mathrm{v}, \mathrm{z}(1, \mathrm{j}), \mathrm{z}(2, \mathrm{j}), \mathrm{z}(3, \mathrm{j}), \mathrm{x} 0, \mathrm{y} 0, \mathrm{z} 0, \mathrm{z}(4, \mathrm{j}), \mathrm{vm}, \mathrm{si}, \mathrm{sx}, \mathrm{sy})$; calextra $=$ calextra + ; end 
$\%$ keep z

final_z=z;

$\% \% \% \% \% \% \% \% \%$ known signal $\% \% \% \% \% \% \% \% \% \% \% \% \% \% \% \% \% \% \% \% \% \% \% \% \%$ load phalf; $\% 25$ fibers

$\mathrm{p}=$ phalf;

$\%$ retrieve $\mathrm{z}$

$\mathrm{z}=$ final_z;

$\%$ generation of real signal from half fibers

test_e $=0$;

$\mathrm{v}=4 ; \mathrm{vm}=1 ; \mathrm{si}=1.01 ; \mathrm{sx}=0.33 ; \mathrm{sy}=0.063 ; \mathrm{n}=5 ; \mathrm{x} 0=0 ; \mathrm{y} 0=0 ; \mathrm{z} 0=0 ;$ test_radius $=5^{*} 10^{\wedge}(-3)$;

for $\mathrm{j}=20: 44$,

$\mathrm{e}=\operatorname{extra}(\mathrm{v}, \mathrm{p}(1, \mathrm{j}), \mathrm{p}(2, \mathrm{j}), \mathrm{p}(3, \mathrm{j}), \mathrm{x} 0, \mathrm{y} 0, \mathrm{z} 0, \mathrm{p}(4, \mathrm{j}), \mathrm{vm}, \mathrm{si}, \mathrm{sx}, \mathrm{sy}) ;$

test_e=test_e+e;

end

figure(1)

$\mathrm{t}=[0.002: 0.01: 2]$;

$1=$ length(test_e);

subplot(1,1,1),plot(t(1:1),calextra(1:1),'r-

',t(1:1),test_e(1:1),'b.'),xlabel('Time(ms)'),ylabel('EAP(mv)'),title('Simulated . Forecasted $-')$

$\% \% \% \% \% \% \% \% \% \% \% \%$

differ=test_e-calextra;

$\% \% \% \%$ calc error $\% \% \% \%$

mdiff=differ;

msquare $=$ mdiff. $\wedge 2$;

msum $=$ sum(msquare);

merror $=$ msum

halfloss $=$ differ;

\section{A.2.4 FUNCTION DIAM2}

function $\operatorname{diam} 2=\operatorname{diam} 2(\mathrm{z})$; 
$\%$ this function is used to simulate myopathic muap (double diameter) and then decompose

\%load final_z to be used as $\mathrm{z}$

\section{\%Add EAPS}

$\%$ generation of the calculated signal from all fibers using $\mathrm{z}$

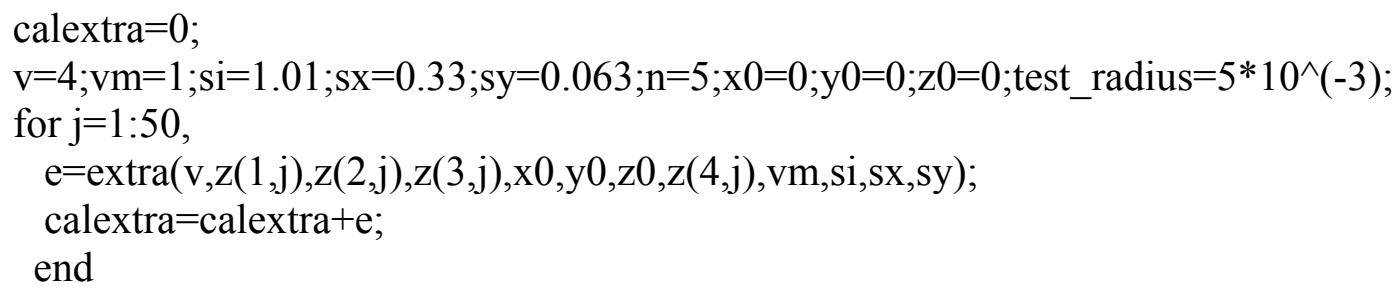




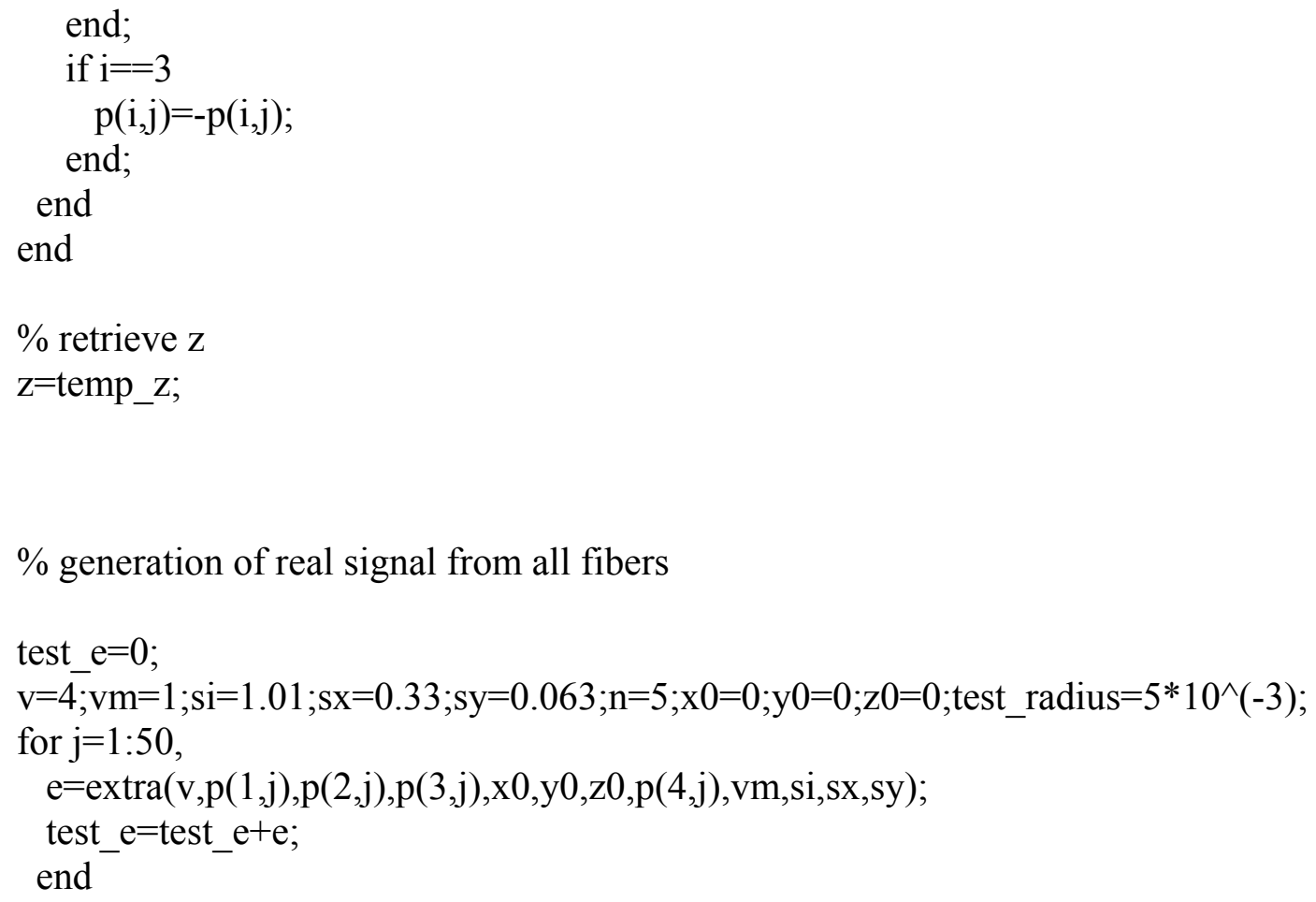

$\%$ generation of real signal from all fibers

test_e $=0$;

$\mathrm{v}=\overline{4 ; \mathrm{vm}}=1 ; \mathrm{si}=1.01 ; \mathrm{sx}=0.33 ; \mathrm{sy}=0.063 ; \mathrm{n}=5 ; \mathrm{x} 0=0 ; \mathrm{y} 0=0 ; \mathrm{z} 0=0 ;$ test_radius $=5^{*} 10^{\wedge}(-3)$; for $\mathrm{j}=1: 50$,

figure(1)

$\mathrm{t}=[0.002: 0.01: 2]$

$1=$ length(test_e);

subplot $(1,1, \overline{1})$, plot(t(1:1), calextra(1:1),'r-

',t(1:1),test_e(1:1),'b.'),xlabel('Time(ms)'),ylabel('EAP(mv)'),title('Simulated . Forecasted $-')$

$\% \% \% \% \% \% \% \% \% \% \% \%$

differ=test_e-calextra;

$\% \% \% \%$ calc error $\% \% \% \% \%$

mdiff= $=$ differ;

msquare $=$ mdiff. $^{\wedge} 2$;

msum $=$ sum(msquare);

merror $=$ msum

$\operatorname{diam} 2=$ differ;

\section{A.2.5 FUNCTION REIN1}

function $\operatorname{rein} 1=\operatorname{rein}(\mathrm{z})$;

$\%$ this function is used to simulate myopathic muap (half fibres are reinervated)

$\%$ in MU's territory and the decompose 
$\% \mathrm{z}$ is created from cr_reiz1 and save as zren1.mat

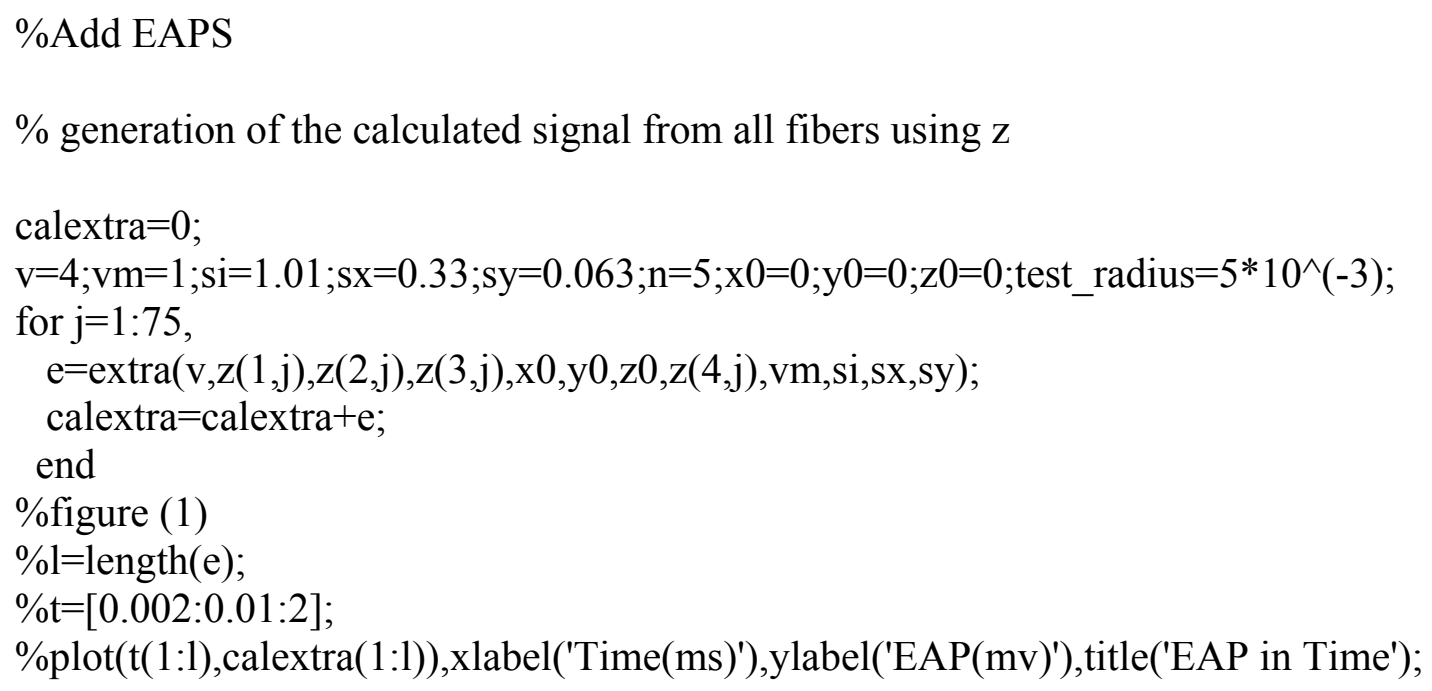




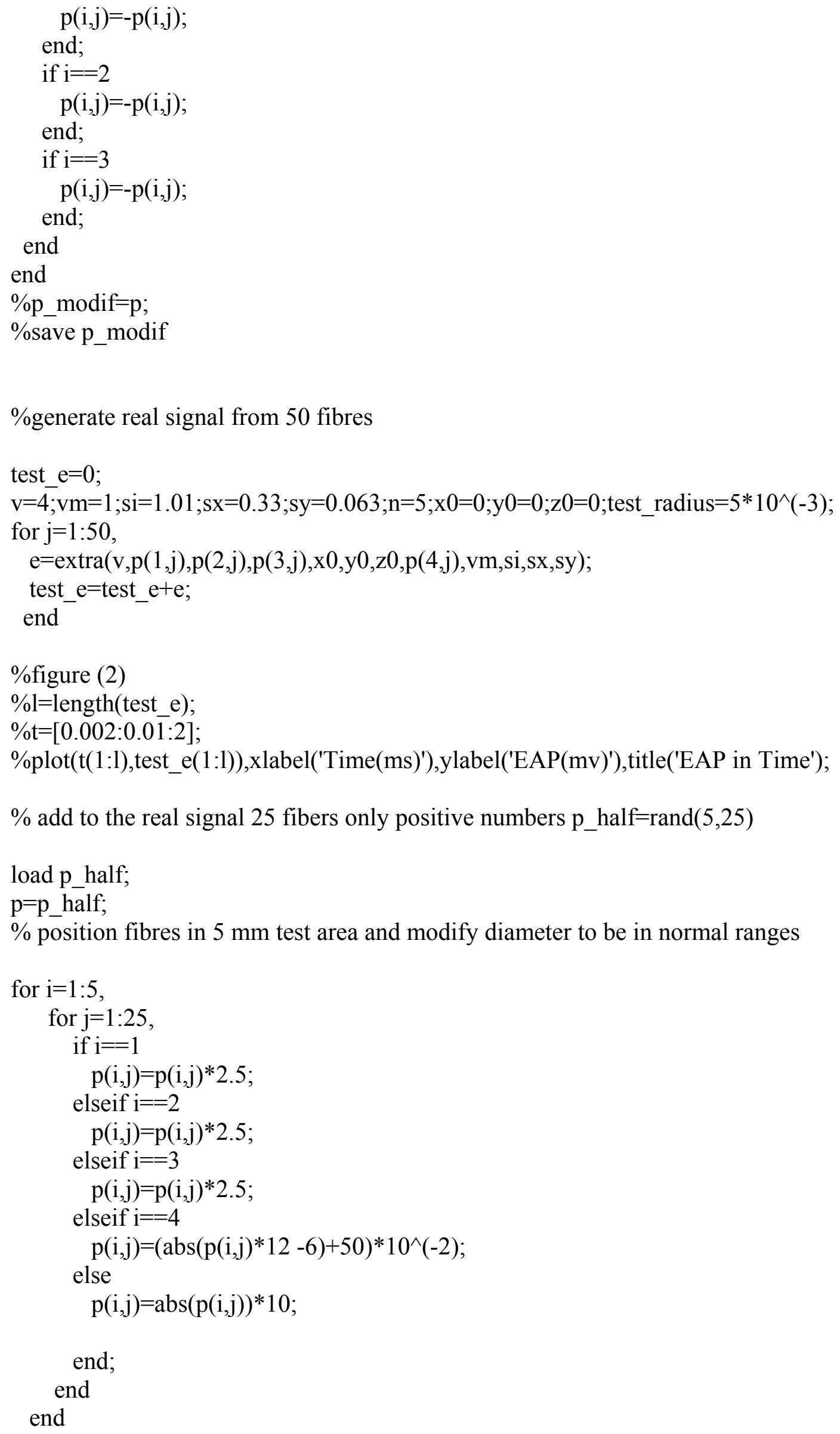




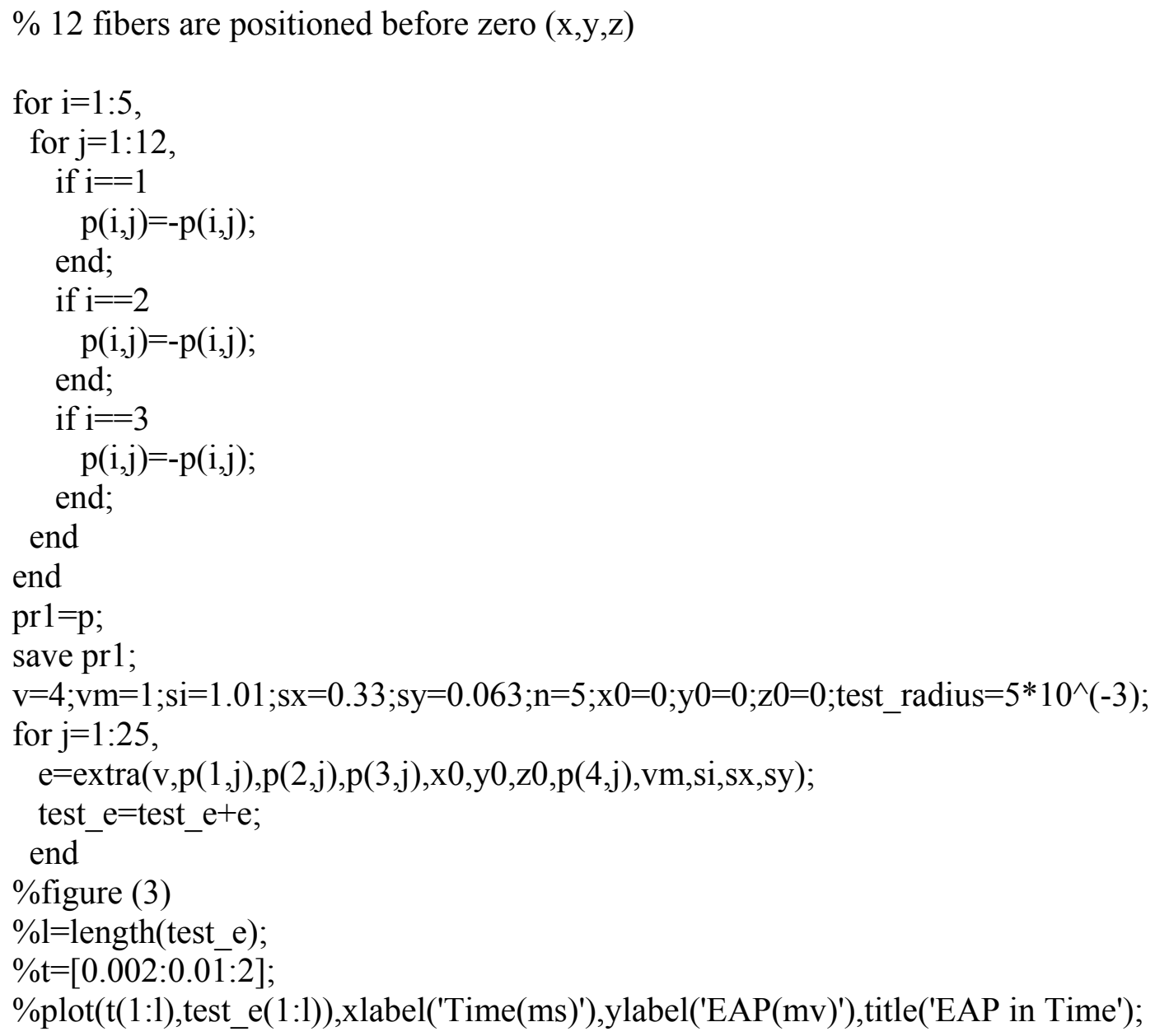

figure(4)

$\mathrm{t}=[0.002: 0.01: 2]$;

$1=$ length(test e);

\%subplot(2,2,1),plot(t(1:1),test_e(1:1)),xlabel('Time(ms)'),ylabel('EAP(mv)'),title('Simul ated EAP in Time');

\%subplot(2,2,2),plot(t(1:1),calextra(1:1)),xlabel('Time(ms)'),ylabel('EAP(mv)'), title('Calc ulated EAP in Time');

$\%$ subplot(2,2,3),plot(t(1:1),calextra(1:1),'y-

',t(1:1),test_e(1:1),'c.'),xlabel('Time(ms)'),ylabel('EAP(mv)'),title('comparison'); subplot $(1,1,1), \operatorname{plot}(\mathrm{t}(1: 1), \operatorname{calextra}(1: 1), \mathrm{r}-$

',t(1:1),test e(1:1),'b.'),xlabel('Time(ms)'),ylabel('EAP(mv)'),title('Simulated . Forecasted $-')$

$\% \% \% \% \% \% \% \% \% \% \% \%$

differ=test_e-calextra;

$\% \% \% \%$ calc error $\% \% \% \% \%$ 
mdiff $=$ differ;

msquare $=$ mdiff. $^{\wedge} 2$;

msum $=$ sum(msquare);

merror $=$ msum

rein $1=$ differ;

\section{A.2.6 FUNCTION REIN2}

function rein $2=\operatorname{rein} 2(z)$;

$\%$ this function is used to simulate myopathic muap (half fibres are reinervated)

$\%$ in MU's territory and the decompose

$\% \mathrm{z}$ is created from cr reiz 2 and save as zren2.mat

\section{$\%$ Add EAPS}

$\%$ generation of the calculated signal from all fibers using $\mathrm{z}$

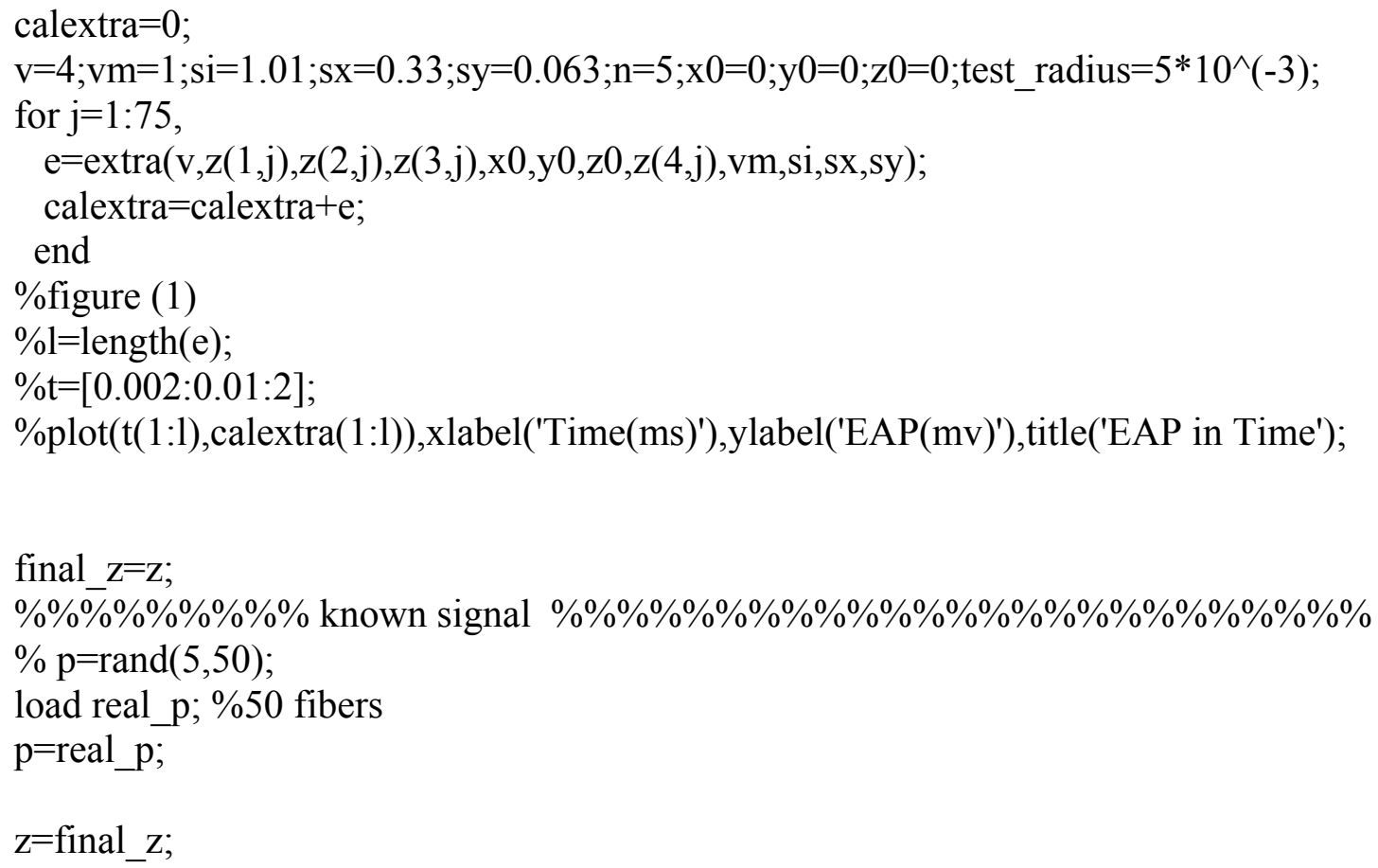




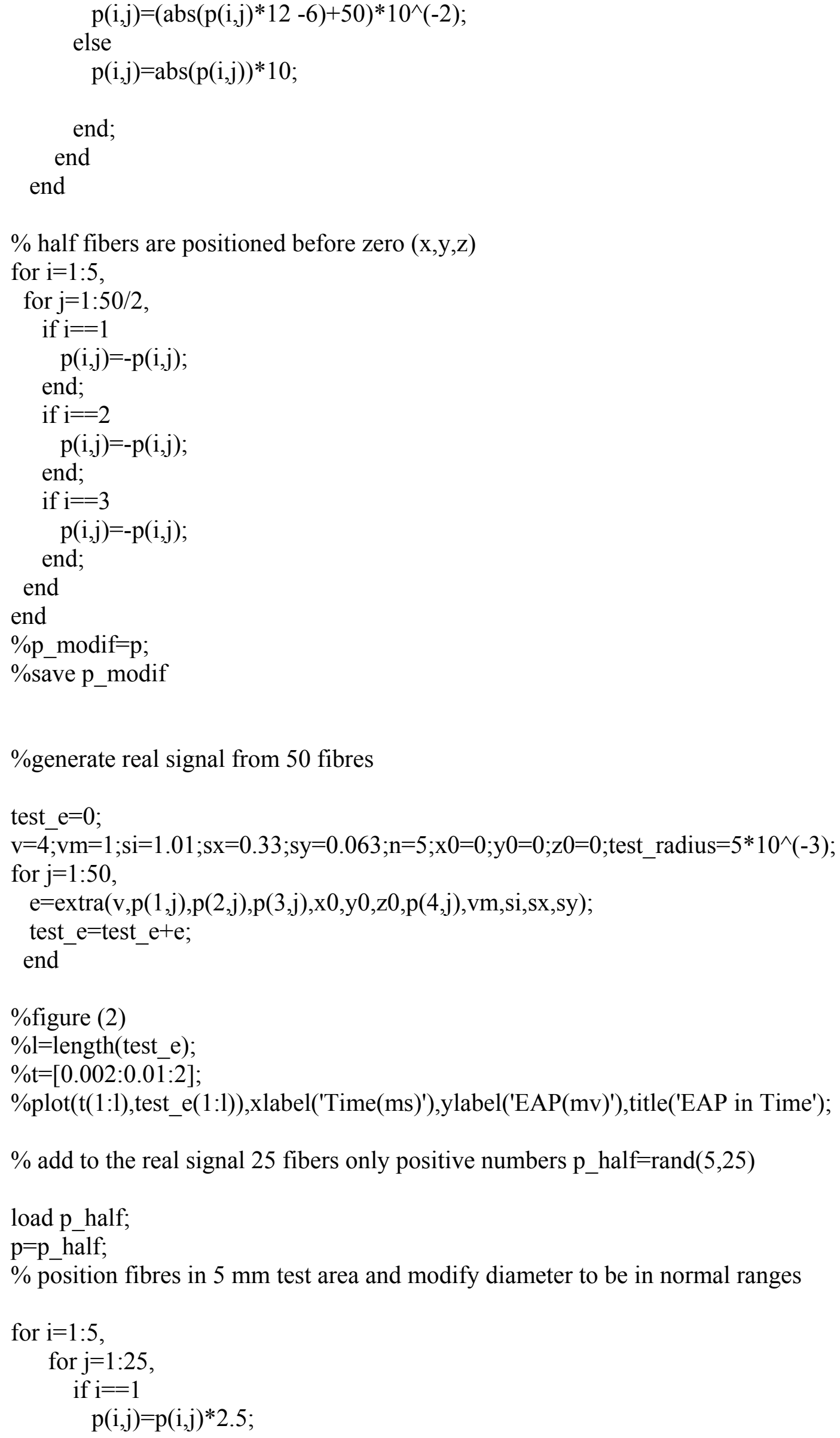




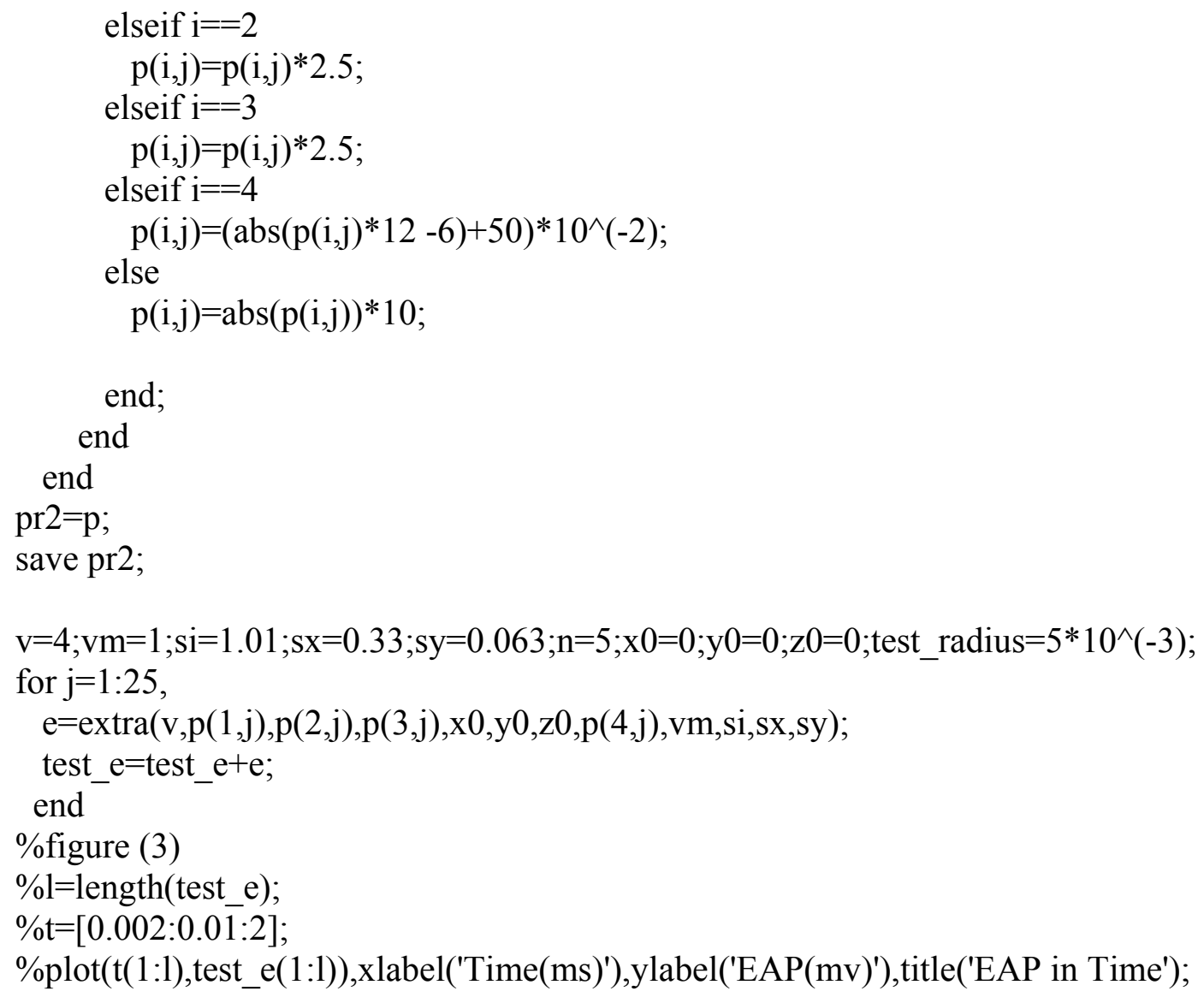




\section{A.2.7 FUNCTION EXTRA}

function extra $=\operatorname{extra}(\mathrm{v}, \mathrm{x} 1, \mathrm{y} 1, \mathrm{z} 1, \mathrm{x} 0, \mathrm{y} 0, \mathrm{z} 0, \mathrm{~d}, \mathrm{vm}, \mathrm{si}, \mathrm{sx}, \mathrm{sy})$

$\%$ function that calculates extra cellular action potential ... Jul 1996

$\% \mathrm{x} 1, \mathrm{y} 1, \mathrm{z} 1$ coordinates of end plate

$\% \mathrm{x} 0, \mathrm{y} 0, \mathrm{z} 0$ coordinates of electrode

$\%$ after Bulgaria

kan $=5$;

$\%$ fibre length in $\mathrm{mm}$

$1=100$;

$\mathrm{b}=5$;

extra $=[]$;

$\%$ for $\mathrm{t}=0: 1.375:(1 / 2+\mathrm{b}) / \mathrm{v}$,

$\mathrm{f}=\mathrm{conv}(\mathrm{fi}$ diff(v),ran_diff(v,x1,y1,z1,x0,y0,z0));

extra $=[$ extra;f];

$\%$ end

$\mathrm{ce}=\mathrm{cl}(\mathrm{d}, \mathrm{kan}, \mathrm{vm}, \mathrm{si}, \mathrm{sx}, \mathrm{sy})$;

extra=-ce*extra;

l=length(extra);

$\%$ plot

$\% \mathrm{t}=[0.002: 0.01: 2]$

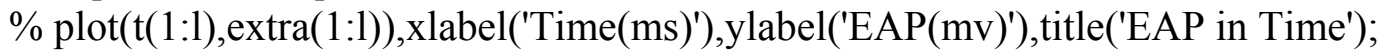

\section{A.2.8 FUNCTION FI_DIFF}

function fi_diff=fi_diff(v)

$\%$ this function calculates the first time derivative of

$\%$ intracellular action potential

$\%$ intracellular : $\mathrm{fi}={ }^{\prime} \mathrm{a} 1^{*} \mathrm{x}^{\wedge} \mathrm{a} 2 * \exp (-\mathrm{a} 3 * \mathrm{x})^{\prime}$

$\%$ fi_diff $=a 1 * x^{\wedge} a 2 * a 2 / x^{*} \exp (-a 3 * x)-a{ }^{*} x^{\wedge} a 2 * a 3 * \exp \left(-a 3^{*} x\right)$

$\%$ coefficients :

$\mathrm{a} 1=9.179951 ; \mathrm{a} 2=4.813291 ; \mathrm{a} 3=2.806631$;

$\%$ for

$\%$ asymmetry kas $=3.5, \mathrm{~b}=5 \mathrm{~mm}$

$\% \mathrm{v}$ is input as $4 \mathrm{~m} / \mathrm{s}$

$\%$ the step :

$\mathrm{dt}=0.02$;

fi_diff_ $\mathrm{x}=[]$;

$\%$ here the spatial derivative is computed

for $\mathrm{t}=0.001: 0.02: 2$,

$\mathrm{x}=\mathrm{v}^{*} \mathrm{t}$;

$\mathrm{f} 1=\mathrm{a} 1 * \mathrm{x}^{\wedge} \mathrm{a} 2$

$\mathrm{f} 2=\mathrm{a} 2 / \mathrm{x}$

$\mathrm{f} 3=\exp (-\mathrm{a} 3 * \mathrm{x})$

$\mathrm{f} 4=\mathrm{a} 1 * \mathrm{x}^{\wedge} \mathrm{a} 2 * \mathrm{a} 3$

$\mathrm{f} 5=\exp (-\mathrm{a} 3 * \mathrm{x})$;

ftotal $=\mathrm{f} 1 * \mathrm{f} 2 . * \mathrm{f} 3-\mathrm{f} 4 . * \mathrm{f} 5$;

fi_diff_x=[fi_diff_x;ftotal]; 
end

\%temporal derivative fi_diff $=\mathrm{fi}$ _diff_ $\mathrm{x} * \mathrm{v} * \mathrm{dt}$, where $\mathrm{dt}=0.02$ the step

fi_diff $=\mathrm{v}^{*} \mathrm{dt} *$ fi_diff_x;

$\% \mathrm{t}=[0.001: 0.0 \overline{2}: 2]$;

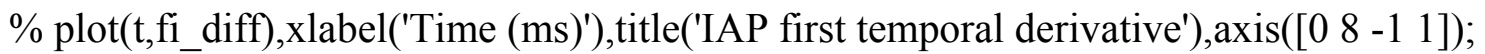

\section{A.2.9 FUNCTION RAN DIFF}

function ran diff=ran diff(v, $\mathrm{x} 1, \mathrm{y} 1, \mathrm{z} 1, \mathrm{x} 0, \mathrm{y} 0, \mathrm{z} 0)$

$\%$ this function calculates the first time derivative of

$\%$ distance of electrode $(\mathrm{x} 0, \mathrm{y} 0, \mathrm{z} 0)$ from the end plate $(\mathrm{x} 1, \mathrm{y} 1, \mathrm{z} 1)$

$\%$ Anisotropicity of the medium :

$\mathrm{kan}=5$;

$\% \mathrm{x} 0, \mathrm{y} 0, \mathrm{z} 0:$ the coordinates of electrode from motor center

$\% \mathrm{x}, \mathrm{y}, \mathrm{z}:$ : the coordinates of signal propagates along the fibre

$\% \quad$ from motor center

$\%$ distance : $\operatorname{ran}=^{\prime}\left((\mathrm{x}+\mathrm{x} 1-\mathrm{x} 0)^{\wedge} 2+\mathrm{kan}(\mathrm{y} 1-\mathrm{y} 0)^{\wedge} 2+\mathrm{kan}(\mathrm{z} 1-\mathrm{z} 0)^{\wedge} 2\right)^{\wedge} 0.5^{\prime}$

$\%$ first spatial derivative of $1 /$ ran :

$\%$ ran_diff $={ }^{\prime}-.5 /\left((\mathrm{x}+\mathrm{x} 1-\mathrm{x} 0)^{\wedge} 2+\mathrm{kan} *(\mathrm{y} 1-\mathrm{y} 0)^{\wedge} 2+\mathrm{kan} *(\mathrm{z} 1-\mathrm{z} 0)^{\wedge} 2\right)^{\wedge} 1.5 *(2 * \mathrm{x}+2 * \mathrm{x} 1-2 * \mathrm{x} 0)^{\prime}$

$\%$ the step :

$\mathrm{dt}=0.02$;

ran_diff_x $=[]$;

$\%$ here the spatial derivative is computed

for $\mathrm{t}=0.001: 0.02: 2$,

$\mathrm{x}=\mathrm{v} * \mathrm{t}$;

$\mathrm{f} 1=-.5 /\left((\mathrm{x}+\mathrm{x} 1-\mathrm{x} 0)^{\wedge} 2+\mathrm{kan}^{*}(\mathrm{y} 1-\mathrm{y} 0)^{\wedge} 2+\mathrm{kan}^{*}(\mathrm{z} 1-\mathrm{z} 0)^{\wedge} 2\right)^{\wedge} 1.5$;

$\mathrm{f} 2=(2 * \mathrm{x}+2 * \mathrm{x} 1-2 * \mathrm{x} 0)$;

ftotal $=\mathrm{f} 1 . * \mathrm{f} 2$;

ran_diff_x $=[$ ran_diff_x;ftotal];

end

\%temporal derivative ran_diff=ran_diff_ $\mathrm{x}^{*} \mathrm{v} * \mathrm{dt}$, where $\mathrm{dt}=0.02$ the step ran_diff $=\mathrm{v} * \mathrm{dt} *$ ran_diff_x;

$\% \mathrm{t}=[0.001: 0.02: 2]$;

$\%$ plot(t,ran_diff),xlabel('Time (ms)'),title('1/ran first temporal derivative');

\section{A.2.10 FUNCTION C1}

function $\mathrm{cl}=\mathrm{c} 1(\mathrm{~d}, \mathrm{kan}, \mathrm{vm}, \mathrm{si}, \mathrm{sx}, \mathrm{sy})$

$\% \mathrm{vm}=1$ (IAP amplidute) si,sx,sy (Rosenfalk Values),d (Fibre Diameter)

$\% \quad \mathrm{kan}=5$

$\mathrm{san}=(\mathrm{sx} * \mathrm{sy})^{\wedge} 1 / 2$;

$\mathrm{c} 1=\left(\mathrm{d}^{\wedge} 2^{*} \mathrm{kan}^{*} \mathrm{vm}{ }^{*} \mathrm{si}\right) /\left(16^{*} \mathrm{san}\right)$; 

APPENDIX 3

TABLES WITH SIMULATED NORMAL MUAPS FOR THREE-DIMENSIONAL MODELS 
Simulated values $\mathrm{x}, \mathrm{y}, \mathrm{z}, \mathrm{d}$ in $\mathrm{mm}, \mathrm{t}$ in $\mathrm{ms}$ (p_modif.mat)

Fibre No:

\begin{tabular}{lllllllllllllll} 
& 1 & 2 & 3 & 4 & 5 & 6 & 7 & 8 & 9 & 10 & 11 & 12 & 13 & 14 \\
\hline $\mathrm{x}$ & -0.5474 & -0.9588 & -1.3243 & -1.0437 & -1.3173 & -2.2758 & -0.8206 & -0.6176 & -0.1817 & -1.9162 & -0.4163 & -2.2616 & -1.2349 & -1.2518 \\
$\mathrm{y}$ & -0.1176 & -1.2985 & -1.6779 & -1.7169 & -0.2299 & -1.9055 & -1.5816 & -2.4564 & -1.5791 & -1.1943 & -1.2163 & -1.2613 & -0.6654 & -0.9604 \\
$\mathrm{z}$ & -1.6972 & -2.0774 & -0.0192 & -1.4724 & -1.6348 & -0.6561 & -1.8910 & -1.8067 & -2.2118 & -0.5944 & -2.2441 & -1.2907 & -0.2268 & -0.6927 \\
$\mathrm{~d}$ & 0.5215 & 0.5559 & 0.5140 & 0.5517 & 0.5101 & 0.5543 & 0.5589 & 0.5304 & 0.5273 & 0.5270 & 0.5491 & 0.5217 & 0.5537 & 0.5497 \\
$\mathrm{t}$ & 9.3469 & 0.5346 & 0.6684 & 8.4617 & 7.0119 & 7.3608 & 3.6534 & 6.5152 & 4.3641 & 3.5926 & 0.6056 & 9.8664 & 0.7375 & 5.2975 \\
\hline
\end{tabular}
Fibre No:

\begin{tabular}{cccccccccccccccc}
\multicolumn{1}{c}{15} & 16 & 17 & 18 & 19 & 20 & 21 & 22 & 23 & 24 & 25 & 26 & 27 & 28 \\
\hline $\mathrm{x}$ & -1.1611 & -2.0695 & -1.5739 & -0.5830 & -2.1150 & -1.3433 & -1.4291 & -2.3884 & -2.1051 & -0.2275 & -1.7745 & 2.2175 & 0.9693 & 1.4753 \\
$\mathrm{y}$ & -2.3524 & -0.3134 & -1.8406 & -0.7658 & -1.0302 & -1.1698 & -2.0060 & -1.8707 & -0.3994 & -0.6865 & -2.3447 & 1.6301 & 1.2494 & 2.3885 \\
$\mathrm{z}$ & -0.1252 & -0.0397 & -1.8135 & -0.8775 & -2.1038 & -0.7180 & -0.0826 & -1.3865 & -0.5319 & -0.0075 & -0.5998 & 0.3758 & 0.3688 & 1.3904 \\
$\mathrm{~d}$ & 0.5314 & 0.5226 & 0.5599 & 0.5016 & 0.5277 & 0.5386 & 0.5041 & 0.5469 & 0.5258 & 0.5103 & 0.5383 & 0.5218 & 0.5105 & 0.5422 \\
$\mathrm{t}$ & 7.7020 & 8.6825 & 8.8857 & 5.9111 & 4.1539 & 1.5372 & 4.9848 & 6.2485 & 1.3043 & 0.2688 & 3.1754 & 3.8581 & 8.4558 & 9.8331 \\
\hline
\end{tabular}
Fibre No:

\begin{tabular}{cccccccccccccccccc}
\multicolumn{2}{r}{3} & 30 & 31 & 32 & 33 & 34 & 35 & 36 & 37 & 38 & 39 & 40 & 41 & 42 \\
\hline $\mathrm{x}$ & 1.0219 & 1.1601 & 1.5732 & 0.6196 & 2.2542 & 0.3280 & 0.9133 & 0.8738 & 0.5381 & 1.1782 & 1.1556 & 1.7225 & 0.7233 & 1.4418 \\
$\mathrm{y}$ & 0.3545 & 2.4027 & 0.3168 & 1.1911 & 1.0662 & 2.2141 & 0.6326 & 1.1308 & 1.6990 & 1.2649 & 2.3784 & 1.7555 & 1.3436 & 2.1914 \\
$\mathrm{z}$ & 1.4122 & 0.3151 & 1.6281 & 0.9733 & 0.3551 & 0.2304 & 0.3378 & 2.0224 & 2.2723 & 1.5010 & 1.5818 & 2.4679 & 1.2861 & 1.1001 \\
$\mathrm{~d}$ & 0.5297 & 0.5360 & 0.5146 & 0.5356 & 0.5537 & 0.5405 & 0.5340 & 0.5518 & 0.5300 & 0.5381 & 0.5073 & 0.5545 & 0.5476 & 0.5276 \\
$\mathrm{t}$ & 4.8851 & 3.1925 & 8.0307 & 0.2838 & 4.1031 & 0.7106 & 4.5531 & 6.5165 & 8.6086 & 7.5584 & 8.2470 & 8.5127 & 4.1403 & 8.6926 \\
\hline
\end{tabular}
Fibre No:

\begin{tabular}{llllllllll}
\multicolumn{1}{l}{43} & \multicolumn{2}{c}{44} & 45 & 46 & 47 & 48 & 49 & 50 \\
\hline $\mathrm{x}$ & 1.7891 & 2.2151 & 1.2224 & 2.1647 & 2.4734 & 2.2038 & 0.5288 & 0.0015 \\
$\mathrm{y}$ & 2.0018 & 1.3125 & 1.6692 & 2.2250 & 0.5388 & 1.0993 & 2.4978 & 0.0022 \\
$\mathrm{z}$ & 1.7663 & 1.1583 & 1.7051 & 1.3599 & 1.1151 & 1.1688 & 0.3840 & 1.9334 \\
$\mathrm{~d}$ & 0.5290 & 0.5522 & 0.5361 & 0.5433 & 0.5221 & 0.5368 & 0.5157 & 0.5273 \\
$\mathrm{t}$ & 0.1909 & 7.1342 & 9.1663 & 4.5035 & 5.1466 & 3.6516 & 6.1635 & 3.1918 \\
\hline
\end{tabular}

Table A.3.1 Normal MUAP - Simulated values for 50 fibres. 


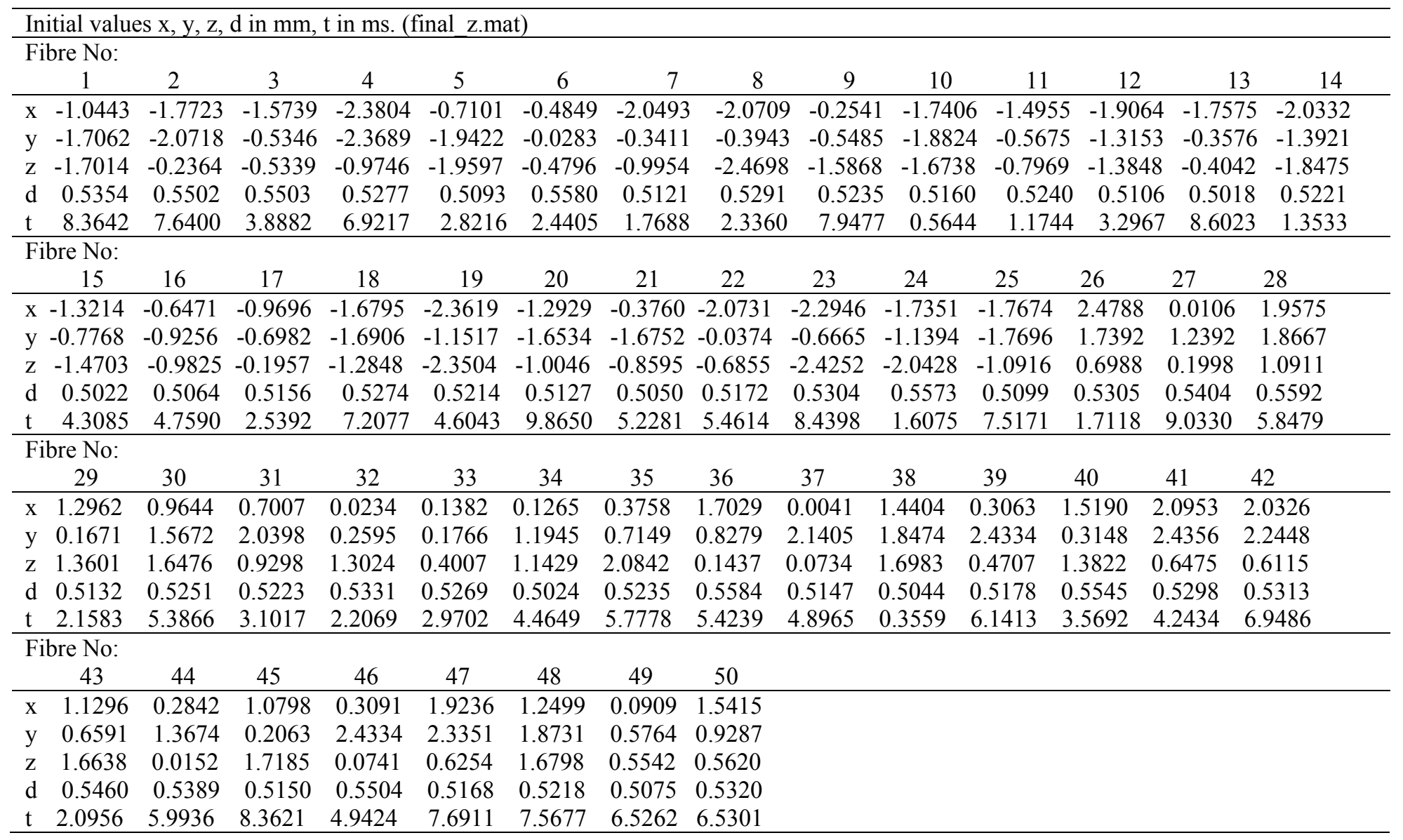

Table A.3.2 Normal MUAP - Initial values for 50 fibres. 


\begin{tabular}{|c|c|c|c|c|c|c|c|c|c|c|c|c|c|c|}
\hline \multicolumn{15}{|c|}{ Results after 251 iterations $\mathrm{x}, \mathrm{y}, \mathrm{z}, \mathrm{d}$ in $\mathrm{mm}, \mathrm{t}$ in $\mathrm{ms}$ (o_no100.mat) } \\
\hline \multicolumn{15}{|c|}{ Fibre No: } \\
\hline & 1 & 2 & 3 & 4 & 5 & 6 & 7 & 8 & 9 & 10 & 11 & 12 & 13 & 14 \\
\hline $\mathrm{x}$ & -1.0443 & -1.7723 & -1.5739 & -2.3804 & -0.7101 & -0.4849 & -2.0493 & -2.0709 & -0.2541 & -1.7406 & -1.4955 & -1.9064 & -1.7575 & -2.0332 \\
\hline $\mathrm{y}$ & -1.7062 & -2.0718 & -0.5346 & -2.3689 & -1.9422 & -0.0283 & -0.3411 & -0.3943 & -0.5485 & -1.8824 & -0.5675 & -1.3153 & -0.3576 & -1.3921 \\
\hline $\mathrm{z}$ & -1.7014 & -0.2364 & -0.5339 & -0.9746 & -1.9597 & -0.4796 & -0.9954 & -2.4698 & -1.5868 & -1.6738 & -0.7969 & -1.3848 & -0.4042 & -1.8475 \\
\hline $\mathrm{d}$ & 0.5354 & 0.5502 & 0.5503 & 0.5277 & 0.5093 & 0.5580 & 0.5121 & 0.5291 & 0.5235 & 0.5160 & 0.5240 & 0.5106 & 0.5018 & 0.5221 \\
\hline $\mathrm{t}$ & 8.3642 & 7.6400 & 3.8882 & 6.9217 & 2.8216 & 2.4405 & 1.7688 & 2.3360 & 7.9477 & 0.5644 & 1.1744 & 3.2967 & 8.6023 & 1.3533 \\
\hline \multicolumn{15}{|c|}{ Fibre No: } \\
\hline & 15 & 16 & 17 & 18 & 19 & 20 & 21 & 22 & 23 & 24 & 25 & 26 & 27 & 28 \\
\hline $\mathrm{x}$ & -1.3214 & -0.6471 & -0.9696 & -1.6795 & -2.3619 & -1.2929 & -0.3760 & -2.0731 & -2.2946 & -1.7351 & -1.7674 & 2.4788 & 0.0106 & 1.9575 \\
\hline $\mathrm{y}$ & -0.7768 & -0.9256 & -0.6982 & -1.6906 & -1.1517 & -1.6534 & -1.6752 & -0.0374 & -0.6665 & -1.1394 & -1.7696 & 1.7392 & 1.2392 & 1.8667 \\
\hline $\mathrm{z}$ & -1.4703 & -0.9825 & -0.1957 & -1.2848 & -2.3504 & -1.0046 & -0.8595 & -0.6855 & -2.4252 & -2.0428 & -1.0916 & 0.6988 & 0.1998 & 1.0911 \\
\hline $\mathrm{d}$ & 0.5022 & 0.5064 & 0.5156 & 0.5274 & 0.5214 & 0.5127 & 0.5050 & 0.5172 & 0.5304 & 0.5573 & 0.5099 & 0.5305 & 0.5404 & 0.5592 \\
\hline $\mathrm{t}$ & 4.3085 & 4.7590 & 2.5392 & 7.2077 & 4.6043 & 9.8650 & 5.2281 & 5.4614 & 8.4398 & 1.6075 & 7.5171 & 1.7118 & 9.0330 & 5.8479 \\
\hline \multicolumn{15}{|c|}{ Fibre No: } \\
\hline & 29 & 30 & 31 & 32 & 33 & 34 & 35 & 36 & 37 & 38 & 39 & 40 & 41 & 42 \\
\hline $\mathrm{x}$ & 1.2962 & 0.9644 & 0.7007 & 0.0234 & 0.1382 & 0.1265 & 0.3758 & 1.7029 & 0.0041 & 1.4404 & 0.3063 & 1.5190 & 2.0953 & 2.0326 \\
\hline $\mathrm{y}$ & 0.1671 & 1.5672 & 2.0398 & 0.2595 & 0.1766 & 1.1945 & 0.7149 & 0.8279 & 2.1405 & 1.8474 & 2.4334 & 0.3148 & 2.4356 & 2.2448 \\
\hline $\mathrm{z}$ & 1.3601 & 1.6476 & 0.9298 & 1.3024 & 0.4007 & 1.1429 & 2.0842 & 0.1437 & 0.0734 & 1.6983 & 0.4707 & 1.3822 & 0.6475 & 0.6115 \\
\hline $\mathrm{d}$ & 0.5132 & 0.5251 & 0.5223 & 0.5331 & 0.5269 & 0.5024 & 0.5235 & 0.5584 & 0.5147 & 0.5044 & 0.5178 & 0.5545 & 0.5298 & 0.5313 \\
\hline $\mathrm{t}$ & 2.1583 & 5.3866 & 3.1017 & 2.2069 & 2.9702 & 4.4649 & 5.7778 & 5.4239 & 4.8965 & 0.3559 & 6.1413 & 3.5692 & 4.2434 & 6.9486 \\
\hline \multicolumn{15}{|c|}{ Fibre No: } \\
\hline & 43 & 44 & 45 & 46 & 47 & 48 & 49 & 50 & & & & & & \\
\hline $\mathrm{x}$ & 1.1296 & 0.2842 & 1.0798 & 0.3091 & 1.9236 & 1.2499 & 0.0909 & 1.5415 & & & & & & \\
\hline $\mathrm{y}$ & 0.6591 & 1.3674 & 0.2063 & 2.4334 & 2.3351 & 1.8731 & 0.5764 & 0.9287 & & & & & & \\
\hline $\mathrm{z}$ & 1.6638 & 0.0152 & 1.7185 & 0.0741 & 0.6254 & 1.6798 & 0.5542 & 0.5620 & & & & & & \\
\hline $\mathrm{d}$ & 0.5460 & 0.5389 & 0.5150 & 0.5504 & 0.5168 & 0.5218 & 0.5075 & 0.5320 & & & & & & \\
\hline $\mathrm{t}$ & 2.0956 & 5.9936 & 8.3621 & 4.9424 & 7.6911 & 7.5677 & 6.5262 & 6.5301 & & & & & & \\
\hline
\end{tabular}

Table A.3.3 Normal MUAP - Results for 50 fibres after 251 iterations. 


\begin{tabular}{|c|c|c|c|c|c|c|c|c|c|c|c|c|c|c|}
\hline \multicolumn{15}{|c|}{ Results after 1011 iterations $\mathrm{x}, \mathrm{y}, \mathrm{z}, \mathrm{d}$ in $\mathrm{mm}, \mathrm{t}$ in $\mathrm{ms}$ (o_no1000.mat) } \\
\hline \multicolumn{15}{|c|}{ Fibre No: } \\
\hline & 1 & 2 & 3 & 4 & 5 & 6 & 7 & 8 & 9 & 10 & 11 & 12 & 13 & 14 \\
\hline $\mathrm{x}$ & $-1,0463$ & $-1,7762$ & $-1,6261$ & $-2,3828$ & $-0,7113$ & $-0,4027$ & $-2,0610$ & $-2,0733$ & $-0,2556$ & $-1,7427$ & $-1,5130$ & $-1,9107$ & $-1,9781$ & $-2,0361$ \\
\hline $\mathrm{y}$ & $-1,7078$ & $-2,0739$ & $-0,5633$ & $-2,3674$ & $-1,9430$ & $-0,0351$ & $-0,3429$ & $-0,3942$ & $-0,5519$ & $-1,8825$ & $-0,5840$ & $-1,3171$ & $-0,2405$ & $-1,3921$ \\
\hline $\mathrm{z}$ & $-1,7029$ & $-0,2366$ & $-0,5625$ & $-0,9740$ & $-1,9605$ & $-0,5958$ & $-1,0006$ & $-2,4692$ & $-1,5966$ & $-1,6739$ & $-0,8201$ & $-1,3867$ & $-0,2719$ & $-1,8475$ \\
\hline $\mathrm{d}$ & 0,5368 & 0,5567 & 0,4803 & 0,5407 & 0,5108 & 0,3277 & 0,5007 & 0,5396 & 0,5034 & 0,5234 & 0,4764 & 0,5171 & 0,5247 & 0,5323 \\
\hline $\mathrm{t}$ & 8,3642 & 7,6400 & 3,8882 & 6,9217 & 2,8216 & 2,4405 & 1,7688 & 2,3360 & 7,9477 & 0,5644 & 1,1744 & 3,2967 & 8,6023 & 1,3533 \\
\hline \multicolumn{15}{|c|}{ Fibre No: } \\
\hline & 15 & 16 & 17 & 18 & 19 & 20 & 21 & 22 & 23 & 24 & 25 & 26 & 27 & 28 \\
\hline $\mathrm{x}$ & $-1,3254$ & $-0,6497$ & $-0,9796$ & $-1,6828$ & $-2,3640$ & $-1,2961$ & $-0,3776$ & $-2,1240$ & $-2,2971$ & $-1,7381$ & $-1,7707$ & 2,4794 & 0,0118 & 1,9576 \\
\hline $\mathrm{y}$ & $-0,7806$ & $-0,9381$ & $-0,7991$ & $-1,6923$ & $-1,1511$ & $-1,6575$ & $-1,6813$ & $-0,0283$ & $-0,6662$ & $-1,1398$ & $-1,7712$ & 1,7425 & 1,2601 & 1,8700 \\
\hline $\mathrm{z}$ & $-1,4773$ & $-0,9959$ & $-0,2239$ & $-1,2861$ & $-2,3491$ & $-1,0071$ & $-0,8626$ & $-0,5192$ & $-2,4240$ & $-2,0435$ & $-1,0926$ & 0,7001 & 0,2032 & 1,0930 \\
\hline d & 0,4938 & 0,4723 & 0,3838 & 0,5327 & 0,5338 & 0,5105 & 0,4929 & 0,5562 & 0,5430 & 0,5649 & 0,5158 & 0,5160 & 0,4945 & 0,5472 \\
\hline $\mathrm{t}$ & 4,3085 & 4,7590 & 2,5392 & 7,2077 & 4,6043 & 9,8650 & 5,2281 & 5,4614 & 8,4398 & 1,6075 & 7,5171 & 1,7118 & 9,0330 & 5,8479 \\
\hline \multicolumn{15}{|c|}{ Fibre No: } \\
\hline & 29 & 30 & 31 & 32 & 33 & 34 & 35 & 36 & 37 & 38 & 39 & 40 & 41 & 42 \\
\hline $\mathrm{x}$ & 1,2980 & 0,9640 & 0,7002 & 0,0239 & 0,1655 & 0,1260 & 0,3750 & 1,7078 & 0,0030 & 1,4402 & 0,3054 & 1,5209 & 2,0952 & 2,0326 \\
\hline $\mathrm{y}$ & 0,1681 & 1,5697 & 2,0435 & 0,2630 & 0,1649 & 1,2013 & 0,7163 & 0,8355 & 2,1451 & 1,8492 & 2,4362 & 0,3166 & 2,4379 & 2,2476 \\
\hline $\mathrm{z}$ & 1,3681 & 1,6503 & 0,9314 & 1,3202 & 0,3741 & 1,1494 & 2,0883 & 0,1450 & 0,0735 & 1,7000 & 0,4712 & 1,3901 & 0,6481 & 0,6122 \\
\hline d & 0,4840 & 0,5158 & 0,5130 & 0,4931 & 0,4128 & 0,4806 & 0,5144 & 0,5145 & 0,5061 & 0,4975 & 0,5128 & 0,5260 & 0,5223 & 0,5218 \\
\hline $\mathrm{t}$ & 2,1583 & 5,3866 & 3,1017 & 2,2069 & 2,9702 & 4,4649 & 5,7778 & 5,4239 & 4,8965 & 0,3559 & 6,1413 & 3,5692 & 4,2434 & 6,9486 \\
\hline \multicolumn{15}{|c|}{ Fibre No: } \\
\hline & 43 & 44 & 45 & 46 & 47 & 48 & 49 & 50 & & & & & & \\
\hline $\mathrm{x}$ & 1,1300 & 0,2852 & 1,0802 & 0,3081 & 1,9235 & 1,2495 & 0,1024 & 1,5449 & & & & & & \\
\hline$y$ & 0,6616 & 1,3830 & 0,2070 & 2,4368 & 2,3376 & 1,8751 & 0,5987 & 0,9358 & & & & & & \\
\hline $\mathrm{z}$ & 1,6701 & 0,0154 & 1,7251 & 0,0742 & 0,6261 & 1,6815 & 0,5755 & 0,5663 & & & & & & \\
\hline $\mathrm{d}$ & 0,5267 & 0,5017 & 0,4952 & 0,5445 & 0,5086 & 0,5150 & 0,4203 & 0,4948 & & & & & & \\
\hline $\mathrm{t}$ & 2,0956 & 5,9936 & 8,3621 & 4,9424 & 7,6911 & 7,5677 & 6,5262 & 6,5301 & & & & & & \\
\hline
\end{tabular}

Table A.3.4 Normal MUAP - Results for 50 fibres after 1011 iterations. 


\begin{tabular}{|c|c|c|c|c|c|c|c|c|c|c|c|c|c|}
\hline \multicolumn{14}{|c|}{ Results after 5065 iterations $\mathrm{x}, \mathrm{y}, \mathrm{z}, \mathrm{d}$ in $\mathrm{mm}, \mathrm{t}$ in $\mathrm{ms}$ (o_no5000.mat) } \\
\hline \multicolumn{14}{|l|}{ Fibre No: } \\
\hline 1 & 2 & 3 & 4 & 5 & 6 & 7 & 8 & 9 & 10 & 11 & 12 & 13 & 14 \\
\hline$-1,0452$ & $-1,7750$ & $-1,5897$ & $-2,3799$ & $-0,7103$ & $-0,4605$ & $-2,0648$ & $-2,0710$ & $-0,2552$ & $-1,7410$ & $-1,5053$ & $-1,9105$ & $-2,0716$ & $-2,0340$ \\
\hline$-1,7046$ & $-2,0646$ & $-0,5722$ & $-2,3638$ & $-1,9411$ & $-0,0379$ & $-0,3533$ & $-0,3935$ & $-0,5506$ & $-1,8794$ & $-0,5846$ & $-1,3097$ & $-0,2051$ & $-1,3881$ \\
\hline$-1,6997$ & $-0,2356$ & $-0,5714$ & $-0,9726$ & $-1,9586$ & $-0,6416$ & $-1,0307$ & $-2,4646$ & $-1,5929$ & $-1,6712$ & $-0,8210$ & $-1,3789$ & $-0,2318$ & $-1,8422$ \\
\hline 0,5483 & 0,5731 & 0,5012 & 0,5410 & 0,5186 & 0,3137 & 0,5307 & 0,5436 & 0,5281 & 0,5293 & 0,5084 & 0,5381 & 0,4937 & 0,5403 \\
\hline 8,3642 & 7,6400 & 3,8882 & 6,9217 & 2,8216 & 2,4405 & 1,7688 & 2,3360 & 7,9477 & 0,5644 & 1,1744 & 3,2967 & 8,6023 & 1,3533 \\
\hline \multicolumn{14}{|l|}{ Fibre No: } \\
\hline$-1,3265$ & $-0,6506$ & $-0,9774$ & $-1,6817$ & $-2,3614$ & $-1,2959$ & $-0,3772$ & $-2,0538$ & $-2,2944$ & $-1,7362$ & $-1,7698$ & 2,4794 & 0,0127 & 1,9580 \\
\hline$-0,7776$ & $-0,9362$ & $-0,7368$ & $-1,6861$ & $-1,1496$ & $-1,6517$ & $-1,6776$ & $-0,0299$ & $-0,6650$ & $-1,1366$ & $-1,7644$ & 1,7366 & 1,2619 & 1,8654 \\
\hline$-1,4717$ & $-0,9939$ & $-0,2065$ & $-1,2814$ & $-2,3461$ & $-1,0036$ & $-0,8607$ & $-0,5477$ & $-2,4196$ & $-2,0378$ & $-1,0884$ & 0,6977 & 0,2035 & 1,0903 \\
\hline 0,5199 & 0,4998 & 0,4485 & 0,5477 & 0,5336 & 0,5305 & 0,5138 & 0,4225 & 0,5448 & 0,5748 & 0,5312 & 0,5447 & 0,5223 & 0,5710 \\
\hline 4,3085 & 4,7590 & 2,5392 & 7,2077 & 4,6043 & 9,8650 & 5,2281 & 5,4614 & 8,4398 & 1,6075 & 7,5171 & 1,7118 & 9,0330 & 5,8479 \\
\hline \multicolumn{14}{|l|}{ Fibre No: } \\
\hline 29 & 30 & 31 & 32 & 33 & 34 & 35 & 36 & 37 & 38 & 39 & 40 & 41 & 42 \\
\hline 1,3005 & 0,9646 & 0,7008 & 0,0248 & 0,3232 & 0,1267 & 0,3757 & 1,7075 & 0,0037 & 1,4406 & 0,3061 & 1,5229 & 2,0954 & 2,0329 \\
\hline 1,3583 & 1,6475 & 0,9298 & 1,3202 & 0,5071 & 1,1470 & 2,0846 & 0,1342 & 0,0734 & 1,6979 & 0,4706 & 1,3783 & 0,6473 & 0,6112 \\
\hline 0,5234 & 0,5346 & 0,5316 & 0,5210 & 0,2477 & 0,5054 & 0,5328 & 0,6225 & 0,5241 & 0,5133 & 0,5271 & 0,5687 & 0,5396 & 0,5418 \\
\hline 2,1583 & 5,3866 & 3,1017 & 2,2069 & 2,9702 & 4,4649 & 5,7778 & 5,4239 & 4,8965 & 0,3559 & 6,1413 & 3,5692 & 4,2434 & 6,9486 \\
\hline \multicolumn{14}{|l|}{ Fibre No: } \\
\hline 43 & 44 & 45 & 46 & 47 & 48 & 49 & 50 & & & & & & \\
\hline 1,1311 & 0,2872 & 1,0813 & 0,3089 & 1,9238 & 1,2500 & 0,1075 & 1,5486 & & & & & & \\
\hline 0,6593 & 1,3814 & 0,2064 & 2,4329 & 2,3341 & 1,8726 & 0,6325 & 0,9145 & & & & & & \\
\hline 1,6643 & 0,0154 & 1,7194 & 0,0741 & 0,6251 & 1,6793 & 0,6080 & 0,5534 & & & & & & \\
\hline 0,5554 & 0,5314 & 0,5233 & 0,5603 & 0,5266 & 0,5310 & 0,4144 & 0,5568 & & & & & & \\
\hline 2,0956 & 5,9936 & 8,3621 & 4,9424 & 7,6911 & 7,5677 & 6,5262 & 6,5301 & & & & & & \\
\hline
\end{tabular}

Table A.3.5 Normal MUAP - Results for 50 fibres after 5065 iterations. 
APPENDIX 4

TABLES WITH SIMULATED MYOPATHY MUAPS FOR THREE-DIMENSIONAL MODELS 


\begin{tabular}{|c|c|c|c|c|c|c|c|c|c|c|c|c|}
\hline \multicolumn{13}{|c|}{ Simulated Values $\mathrm{x}, \mathrm{y}, \mathrm{z}, \mathrm{d}$ in $\mathrm{mm}, \mathrm{t}$ in $\mathrm{ms}$ (phalf.mat) } \\
\hline \multicolumn{13}{|c|}{ Fibre No: } \\
\hline $\mathrm{x}-1.3433$ & -1.4291 & -2.3884 & -2.1051 & -0.2275 & -1.7745 & $5 \quad 2.2175$ & $5 \quad 0.9693$ & $3 \quad 1.4753$ & 31.0219 & 1.1601 & 1.5732 & 0.6196 \\
\hline $\mathrm{Z}-0.7180$ & -0.0826 & -1.3865 & -0.5319 & -0.0075 & -0.5998 & 0.3758 & 0.3688 & 1.3904 & $4 \quad 1.4122$ & 20.3151 & 1.6281 & 0.9733 \\
\hline d 0.5386 & 0.5041 & 0.5304 & 0.5273 & 0.5270 & 0.5491 & 0.5217 & $7 \quad 0.5537$ & $7 \quad 0.5497$ & $\begin{array}{ll}7 & 0.529\end{array}$ & $7 \quad 0.5360$ & $0 \quad 0.5146$ & 0.5356 \\
\hline t 1.5372 & 4.9848 & 7.7020 & 8.6825 & 8.8857 & 5.9111 & 4.1539 & $\begin{array}{l}9 \\
9\end{array}$ & 24.9848 & $8 \quad 4.885$ & $1 \quad 3.1925$ & $5 \quad 8.0307$ & 0.2838 \\
\hline X 2.2542 & 0.3280 & 0.9133 & 0.8738 & 0.5381 & 1.1782 & 1.1556 & 1.7225 & 0.7233 & 1.4418 & 1.7891 & 2.2151 & \\
\hline y 1.0662 & 2.2141 & 0.6326 & 1.1308 & 1.6990 & 1.2649 & 2.3784 & 1.7555 & 1.3436 & 2.1914 & 2.0018 & 1.3125 & \\
\hline z $\quad 0.3551$ & 0.2304 & 0.3378 & 2.0224 & 2.2723 & 1.5010 & 1.5818 & 2.4679 & 1.2861 & 1.1001 & 1.7663 & 1.1583 & \\
\hline d $\quad 0.5537$ & 0.5405 & 0.5340 & 0.5518 & 0.5300 & 0.5381 & 0.5073 & 0.5545 & 0.5476 & 0.5276 & 0.5290 & 0.5522 & \\
\hline $\mathrm{t} 4.1031$ & 0.7106 & 4.5531 & 6.5165 & 8.6086 & 7.5584 & 8.2470 & 8.5127 & 4.1403 & 8.6926 & 0.1909 & 7.1342 & \\
\hline
\end{tabular}

Table A.4.1 Myopathy MUAP - Loss of the half fibres, simulated values. 
Initial values $\mathrm{x}, \mathrm{y}, \mathrm{z}, \mathrm{d}$ in $\mathrm{mm}, \mathrm{t}$ in $\mathrm{ms}$ (zhalf.mat)

Fibre No:

20 21 $22 \quad 23$ 24 25 $26 \quad 27$ 28 29 30 31 32

\begin{tabular}{|c|c|c|c|c|c|c|c|c|c|c|c|c|c|}
\hline $\mathrm{x}$ & -1.2929 & -0.3760 & -2.0731 & -2.2946 & -1.7351 & -1.7674 & 2.4788 & 0.0106 & 1.9575 & 1.2962 & 0.9644 & 0.7007 & 0.0234 \\
\hline $\mathrm{y}$ & -1.6534 & -1.6752 & -0.0374 & -0.6665 & -1.1394 & -1.7696 & 1.7392 & 1.2392 & 1.8667 & 0.1671 & 1.5672 & 2.0398 & 0.2595 \\
\hline z & -1.0046 & -0.8595 & -0.6855 & -2.4252 & -2.0428 & -1.0916 & 0.6988 & 0.1998 & 1.0911 & 1.3601 & 1.6476 & 0.9298 & 1.3024 \\
\hline$d$ & 0.5127 & 0.5050 & 0.5172 & 0.5304 & 0.5573 & 0.5099 & 0.5305 & 0.5404 & 0.5592 & 0.5132 & 0.5251 & 0.5223 & 0.5331 \\
\hline $\mathrm{t}$ & 9.8650 & 5.2281 & 5.4614 & 8.4398 & 1.6075 & 7.5171 & 1.7118 & 9.0330 & 5.8479 & 2.1583 & 5.3866 & 3.1017 & 2.2069 \\
\hline \multicolumn{14}{|c|}{ Fibre No: } \\
\hline 33 & 34 & 35 & 36 & 37 & 38 & 39 & 40 & 41 & 42 & 43 & 44 & & \\
\hline $\mathrm{x}$ & 0.1382 & 0.1265 & 0.3758 & 1.7029 & 0.0041 & 1.4404 & 0.3063 & 1.5190 & 2.0953 & 2.0326 & 1.1296 & 0.2842 & \\
\hline $\mathrm{y}$ & 0.1766 & 1.1945 & 0.7149 & 0.8279 & 2.1405 & 1.8474 & 2.4334 & 0.3148 & 2.4356 & 2.2448 & 0.6591 & 1.3674 & \\
\hline $\mathrm{z}$ & 0.4007 & 1.1429 & 2.0842 & 0.1437 & 0.0734 & 1.6983 & 0.4707 & 1.3822 & 0.6475 & 0.6115 & 1.6638 & 0.0152 & \\
\hline d & 0.5269 & 0.5024 & 0.5235 & 0.5584 & 0.5147 & 0.5044 & 0.5178 & 0.5545 & 0.5298 & 0.5313 & 0.5460 & 0.5389 & \\
\hline t & 2.9702 & 4.4649 & 5.7778 & 5.4239 & 4.8965 & 0.3559 & 6.1413 & 3.5692 & 4.2434 & 6.9486 & 2.0956 & 5.9936 & \\
\hline
\end{tabular}

Table A.4.2 Myopathy MUAP - Loss of the half fibres, initial values. 


\begin{tabular}{|c|c|c|c|c|c|c|c|c|c|c|c|c|c|}
\hline \multicolumn{14}{|c|}{ Results after 221 iterations $\mathrm{x}, \mathrm{y}, \mathrm{z}, \mathrm{d}$ in $\mathrm{mm}, \mathrm{t}$ in $\mathrm{ms}(\ln 100 . \mathrm{mat})$} \\
\hline \multicolumn{14}{|c|}{ Fibre No: } \\
\hline & & 21 & 22 & 23 & 24 & 25 & 26 & 27 & 28 & 29 & 30 & 31 & 32 \\
\hline $\mathrm{x}$ & $-1,2929$ & $-0,3760$ & $-2,0731$ & $-2,2946$ & $-1,7351$ & $-1,7674$ & 2,4788 & 0,0106 & 1,9575 & 1,2962 & 0,9644 & 0,7007 & 0,0234 \\
\hline y & $-1,6534$ & $-1,6752$ & $-0,0374$ & $-0,6665$ & $-1,1394$ & $-1,7696$ & 1,7392 & 1,2392 & 1,8667 & 0,1671 & 1,5672 & 2,0398 & 0,2595 \\
\hline $\mathrm{z}$ & $-1,0046$ & $-0,8595$ & $-0,6855$ & $-2,4252$ & $-2,0428$ & $-1,0916$ & 0,6988 & 0,1998 & 1,0911 & 1,3601 & 1,6476 & 0,9298 & 1,3024 \\
\hline d & 0,5127 & 0,5050 & 0,5172 & 0,5304 & 0,5573 & 0,5099 & 0,5305 & 0,5404 & 0,5592 & 0,5132 & 0,5251 & 0,5223 & 0,5331 \\
\hline $\mathrm{t}$ & 9,8650 & 5,2281 & 5,4614 & 8,4398 & 1,6075 & 7,5171 & 1,7118 & 9,0330 & 5,8479 & 2,1583 & 5,3866 & 3,1017 & 2,2069 \\
\hline \multicolumn{14}{|c|}{ Fibre No: } \\
\hline & & 34 & 35 & 36 & 37 & 38 & 39 & 40 & 41 & 42 & 43 & 44 & \\
\hline $\mathrm{x}$ & 0,1382 & 0,1265 & 0,3758 & 1,7029 & 0,0041 & 1,4404 & 0,3063 & 1,5190 & 2,0953 & 2,0326 & 1,1296 & 0,2842 & \\
\hline $\mathrm{y}$ & 0,1766 & 1,1945 & 0,7149 & 0,8279 & 2,1405 & 1,8474 & 2,4334 & 0,3148 & 2,4356 & 2,2448 & 0,6591 & 1,3674 & \\
\hline $\mathrm{z}$ & 0,4007 & 1,1429 & 2,0842 & 0,1437 & 0,0734 & 1,6983 & 0,4707 & 1,3822 & 0,6475 & 0,6115 & 1,6638 & 0,0152 & \\
\hline d & 0,5269 & 0,5024 & 0,5235 & 0,5584 & 0,5147 & 0,5044 & 0,5178 & 0,5545 & 0,5298 & 0,5313 & 0,5460 & 0,5389 & \\
\hline $\mathrm{t}$ & 2,9702 & 4,4649 & 5,7778 & 5,4239 & 4,8965 & 0,3559 & 6,1413 & 3,5692 & 4,2434 & 6,9486 & 2,0956 & 5,9936 & \\
\hline
\end{tabular}

Table A.4.3 Myopathy MUAP - loss of the half fibres, results after 221 iterations. 


\begin{tabular}{|c|c|c|c|c|c|c|c|c|c|c|c|c|c|}
\hline \multicolumn{14}{|c|}{ Results after 1115 iterations $\mathrm{x}, \mathrm{y}, \mathrm{z}, \mathrm{d}$ in $\mathrm{mm}, \mathrm{t}$ in $\mathrm{ms}$ (ln1000.mat) } \\
\hline \multicolumn{14}{|c|}{ Fibre No: } \\
\hline & & 21 & 22 & 23 & 24 & 25 & 26 & 27 & 28 & 29 & 30 & 31 & 32 \\
\hline $\mathrm{x}$ & $-1,2861$ & $-0,3720$ & $-2,1077$ & $-2,2940$ & $-1,7319$ & $-1,7621$ & 2,4790 & 0,0074 & 1,9580 & 1,2948 & 0,9655 & 0,7021 & 0,0222 \\
\hline $\mathrm{y}$ & $-1,6581$ & $-1,6728$ & $-0,0359$ & $-0,6697$ & $-1,1451$ & $-1,7798$ & 1,7385 & 1,2104 & 1,8660 & 0,1667 & 1,5668 & 2,0394 & 0,2549 \\
\hline $\mathrm{z}$ & $-1,0075$ & $-0,8583$ & $-0,6593$ & $-2,4368$ & $-2,0530$ & $-1,0979$ & 0,6985 & 0,1951 & 1,0907 & 1,3566 & 1,6473 & 0,9296 & 1,2792 \\
\hline $\mathrm{d}$ & 0,4757 & 0,4882 & 0,5112 & 0,5026 & 0,5237 & 0,4670 & 0,5268 & 0,5617 & 0,5538 & 0,5147 & 0,5169 & 0,5130 & 0,5466 \\
\hline $\mathrm{t}$ & 9,8650 & 5,2281 & 5,4614 & 8,4398 & 1,6075 & 7,5171 & 1,7118 & 9,0330 & 5,8479 & 2,1583 & 5,3866 & 3,1017 & 2,2069 \\
\hline \multicolumn{14}{|c|}{ Fibre No: } \\
\hline & & 34 & 35 & 36 & 37 & 38 & 39 & 40 & 41 & 42 & 43 & 44 & \\
\hline $\mathrm{x}$ & 0,1167 & 0,1285 & 0,3775 & 1,7020 & 0,0065 & 1,4411 & 0,3077 & 1,5180 & 2,0957 & 2,0331 & 1,1302 & 0,2826 & \\
\hline y & 0,2648 & 1,1886 & 0,7147 & 0,8330 & 2,1402 & 1,8475 & 2,4344 & 0,3142 & 2,4356 & 2,2445 & 0,6578 & 1,3508 & \\
\hline $\mathrm{z}$ & 0,6009 & 1,1373 & 2,0838 & 0,1446 & 0,0734 & 1,6984 & 0,4709 & 1,3792 & 0,6475 & 0,6114 & 1,6606 & 0,0150 & \\
\hline $\mathrm{d}$ & 0,4930 & 0,4972 & 0,5123 & 0,5565 & 0,5008 & 0,4979 & 0,5067 & 0,5544 & 0,5245 & 0,5260 & 0,5415 & 0,5479 & \\
\hline $\mathrm{t}$ & 2,9702 & 4,4649 & 5,7778 & 5,4239 & 4,8965 & 0,3559 & 6,1413 & 3,5692 & 4,2434 & 6,9486 & 2,0956 & 5,9936 & \\
\hline
\end{tabular}

Table A.4.4 Myopathy MUAP - loss of the half fibres, results after 1115 iterations . 
Results after 1113 iterations $\mathrm{x}, \mathrm{y}, \mathrm{z}, \mathrm{d}$ in $\mathrm{mm}, \mathrm{t}$ in $\mathrm{ms}(\ln 2000 . \mathrm{mat}), \mathrm{o} \_\ln 1000 . \mathrm{mat}$ has been used as input Fibre No:

\begin{tabular}{|c|c|c|c|c|c|c|c|c|c|c|c|c|c|}
\hline & & 21 & 22 & 23 & 24 & 25 & 26 & 27 & 28 & 29 & 30 & 31 & 32 \\
\hline $\mathrm{x}$ & $-1,2752$ & $-0,3643$ & $-2,1260$ & $-2,3011$ & $-1,7300$ & $-1,7535$ & 2,4809 & 0,0203 & 1,9600 & 1,3004 & 0,9678 & 0,7048 & 0,0343 \\
\hline $\mathrm{y}$ & $-1,6826$ & $-1,6867$ & $-0,0358$ & $-0,6835$ & $-1,1685$ & $-1,8158$ & 1,7408 & 1,2041 & 1,8709 & 0,1668 & 1,5733 & 2,0491 & 0,2547 \\
\hline z & $-1,0223$ & $-0,8654$ & $-0,6574$ & $-2,4869$ & $-2,0949$ & $-1,1201$ & 0,6994 & 0,1941 & 1,0935 & 1,3574 & 1,6541 & 0,934 & 1,2784 \\
\hline$d$ & 0,4261 & 0,4669 & 0,5102 & 0,5408 & 0,5228 & 0,4207 & 0,5547 & 0,5301 & 0,5835 & 0,5225 & 0,5398 & 0,532 & 0,5158 \\
\hline $\mathrm{t}$ & 9,8650 & 5,2281 & 5,4614 & 8,4398 & 1,6075 & 7,5171 & 1,7118 & 9,0330 & 5,8479 & 2,1583 & 5,3866 & 3,1017 & 2,2069 \\
\hline \multicolumn{14}{|c|}{ Fibre No: } \\
\hline \multicolumn{2}{|c|}{33} & 34 & 35 & 36 & 37 & 38 & 39 & 40 & 41 & 42 & 43 & 44 & \\
\hline $\mathrm{x}$ & 0,0102 & 0,1363 & 0,381 & 1,7102 & 0,0109 & 1,4419 & 0,309 & 1,5236 & 2,0963 & 2,0343 & 1,1351 & 0,2926 & \\
\hline $\mathrm{y}$ & 0,2855 & 1,1948 & 0,7189 & 0,8095 & 2,1552 & 1,8524 & 2,4471 & 0,3143 & 2,4406 & 2,2495 & 0,6602 & 1,3523 & \\
\hline $\mathrm{z}$ & 0,6479 & 1,1431 & 2,0959 & 0,1405 & 0,0739 & 1,7029 & 0,4733 & 1,3799 & 0,6489 & 0,6128 & 1,6666 & 0,015 & \\
\hline d & 0,5033 & 0,4798 & 0,5256 & 0,5895 & 0,5049 & 0,5299 & 0,5333 & 0,5678 & 0,5603 & 0,5578 & 0,5522 & 0,5275 & \\
\hline $\mathrm{t}$ & 2,9702 & 4,4649 & 5,7778 & 5,4239 & 4,8965 & 0,3559 & 6,1413 & 3,5692 & 4,2434 & 6,9486 & 2,0956 & 5,9936 & \\
\hline
\end{tabular}

Table A.4.5 Myopathy MUAP - loss of the half fibres, results after 1113 iterations. 
Simulated values $\mathrm{x}, \mathrm{y}, \mathrm{z}, \mathrm{d}$ in $\mathrm{mm}, \mathrm{t}$ in $\mathrm{ms}$ (pdiam.mat)

Fibre No:

\begin{tabular}{lrrrrrrrrrrrrrrrr} 
& 1 & 2 & 3 & \multicolumn{2}{c}{4} & \multicolumn{2}{c}{5} & 6 & 7 & 8 & 9 & 10 & 11 & 12 & 13 & 14 \\
\hline $\mathrm{x}$ & -0.5474 & -0.9588 & -1.3243 & -1.0437 & -1.3173 & -2.2758 & -0.8206 & -0.6176 & -0.1817 & -1.9162 & -0.4163 & -2.2616 & -1.2349 & -1.2518 \\
$\mathrm{y}$ & -0.1176 & -1.2985 & -1.6779 & -1.7169 & -0.2299 & -1.9055 & -1.5816 & -2.4564 & -1.5791 & -1.1943 & -1.2163 & -1.2613 & -0.6654 & -0.9604 \\
$\mathrm{z}$ & -1.6972 & -2.0774 & -0.0192 & -1.4724 & -1.6348 & -0.6561 & -1.8910 & -1.8067 & -2.2118 & -0.5944 & -2.2441 & -1.2907 & -0.2268 & -0.6927 \\
$\mathrm{~d}$ & 1.0430 & 1.1117 & 1.0280 & 1.1033 & 1.0202 & 1.1086 & 1.1178 & 1.0608 & 1.0545 & 1.0540 & 1.0982 & 1.0434 & 1.1075 & 1.0993 \\
$\mathrm{t}$ & 9.3469 & 0.5346 & 0.6684 & 8.4617 & 7.0119 & 7.3608 & 3.6534 & 6.5152 & 4.3641 & 3.5926 & 0.6056 & 9.8664 & 0.7375 & 5.2975 \\
\hline
\end{tabular}
Fibre No:

\begin{tabular}{llllllllllllllllll}
\multicolumn{1}{c}{15} & 16 & 17 & 18 & 19 & 20 & 21 & 22 & 23 & 24 & 25 & 26 & 27 & 28 \\
\hline $\mathrm{x}$ & -1.1611 & -2.0695 & -1.5739 & -0.5830 & -2.1150 & -1.3433 & -1.4291 & -2.3884 & -2.1051 & -0.2275 & -1.7745 & 2.2175 & 0.9693 & 1.4753 \\
$\mathrm{y}$ & -2.3524 & -0.3134 & -1.8406 & -0.7658 & -1.0302 & -1.1698 & -2.0060 & -1.8707 & -0.3994 & -0.6865 & -2.3447 & 1.6301 & 1.2494 & 2.3885 \\
$\mathrm{z}$ & -0.1252 & -0.0397 & -1.8135 & -0.8775 & -2.1038 & -0.7180 & -0.0826 & -1.3865 & -0.5319 & -0.0075 & -0.5998 & 0.3758 & 0.3688 & 1.3904 \\
$\mathrm{~d}$ & 1.0628 & 1.0452 & 1.1199 & 1.0032 & 1.0554 & 1.0772 & 1.0083 & 1.0938 & 1.0515 & 1.0206 & 1.0766 & 1.0435 & 1.0209 & 1.0844 \\
$\mathrm{t}$ & 7.7020 & 8.6825 & 8.8857 & 5.9111 & 4.1539 & 1.5372 & 4.9848 & 6.2485 & 1.3043 & 0.2688 & 3.1754 & 3.8581 & 8.4558 & 9.8331 \\
\hline
\end{tabular}
Fibre No:

\begin{tabular}{llllllllllllllllll}
\multicolumn{2}{c}{29} & 30 & 31 & 32 & 33 & 34 & 35 & 36 & 37 & 38 & 39 & 40 & 41 & 42 \\
\hline $\mathrm{x}$ & 1.0219 & 1.1601 & 1.5732 & 0.6196 & 2.2542 & 0.3280 & 0.9133 & 0.8738 & 0.5381 & 1.1782 & 1.1556 & 1.7225 & 0.7233 & 1.4418 \\
$\mathrm{y}$ & 0.3545 & 2.4027 & 0.3168 & 1.1911 & 1.0662 & 2.2141 & 0.6326 & 1.1308 & 1.6990 & 1.2649 & 2.3784 & 1.7555 & 1.3436 & 2.1914 \\
$\mathrm{z}$ & 1.4122 & 0.3151 & 1.6281 & 0.9733 & 0.3551 & 0.2304 & 0.3378 & 2.0224 & 2.2723 & 1.5010 & 1.5818 & 2.4679 & 1.2861 & 1.1001 \\
$\mathrm{~d}$ & 1.0595 & 1.0721 & 1.0292 & 1.0712 & 1.1074 & 1.0811 & 1.0680 & 1.1036 & 1.0600 & 1.0762 & 1.0146 & 1.1091 & 1.0952 & 1.0551 \\
$\mathrm{t}$ & 4.8851 & 3.1925 & 8.0307 & 0.2838 & 4.1031 & 0.7106 & 4.5531 & 6.5165 & 8.6086 & 7.5584 & 8.2470 & 8.5127 & 4.1403 & 8.6926 \\
\hline
\end{tabular}
Fibre No:

\begin{tabular}{llllllllll}
\multicolumn{1}{l}{43} & \multicolumn{2}{c}{44} & 45 & 46 & 47 & 48 & 49 & \multicolumn{2}{c}{50} \\
\hline $\mathrm{x}$ & 1.7891 & 2.2151 & 1.2224 & 2.1647 & 2.4734 & 2.2038 & 0.5288 & 0.0015 \\
$\mathrm{y}$ & 2.0018 & 1.3125 & 1.6692 & 2.2250 & 0.5388 & 1.0993 & 2.4978 & 0.0022 \\
$\mathrm{z}$ & 1.7663 & 1.1583 & 1.7051 & 1.3599 & 1.1151 & 1.1688 & 0.3840 & 1.9334 \\
$\mathrm{~d}$ & 1.0580 & 1.1044 & 1.0721 & 1.0866 & 1.0442 & 1.0736 & 1.0313 & 1.0546 \\
$\mathrm{t}$ & 0.1909 & 7.1342 & 9.1663 & 4.5035 & 5.1466 & 3.6516 & 6.1635 & 3.1918
\end{tabular}

Table A.4.6 Myopathy MUAP - diameter variability, simulated values. 
Results after 251 iterations $\mathrm{x}, \mathrm{y}, \mathrm{z}, \mathrm{d}$ in $\mathrm{mm}, \mathrm{t}$ in $\mathrm{ms}$ (o_dn100.mat)

Fibre No:

\begin{tabular}{lrrrrrrrrrrrrrrrrr}
\multicolumn{1}{c}{1} & 2 & 3 & \multicolumn{2}{c}{4} & \multicolumn{2}{c}{5} & 6 & 7 & 8 & 9 & 10 & 11 & 12 & 13 & 14 \\
\hline $\mathrm{x}$ & -1.0443 & -1.7723 & -1.5739 & -2.3804 & -0.7101 & -0.4849 & -2.0493 & -2.0709 & -0.2541 & -1.7406 & -1.4955 & -1.9064 & -1.7575 & -2.0332 \\
$\mathrm{y}$ & -1.7062 & -2.0718 & -0.5346 & -2.3689 & -1.9422 & -0.0283 & -0.3411 & -0.3943 & -0.5485 & -1.8824 & -0.5675 & -1.3153 & -0.3576 & -1.3921 \\
$\mathrm{z}$ & -1.7014 & -0.2364 & -0.5339 & -0.9746 & -1.9597 & -0.4796 & -0.9954 & -2.4698 & -1.5868 & -1.6738 & -0.7969 & -1.3848 & -0.4042 & -1.8475 \\
$\mathrm{~d}$ & 0.5354 & 0.5502 & 0.5503 & 0.5277 & 0.5093 & 0.5580 & 0.5121 & 0.5291 & 0.5235 & 0.5160 & 0.5240 & 0.5106 & 0.5018 & 0.5221 \\
$\mathrm{t}$ & 8.3642 & 7.6400 & 3.8882 & 6.9217 & 2.8216 & 2.4405 & 1.7688 & 2.3360 & 7.9477 & 0.5644 & 1.1744 & 3.2967 & 8.6023 & 1.3533 \\
\hline
\end{tabular}
Fibre No:

\begin{tabular}{llllllllllllllllll}
\multicolumn{1}{c}{15} & 16 & 17 & 18 & 19 & 20 & 21 & 22 & 23 & 24 & 25 & 26 & 27 & 28 \\
\hline $\mathrm{x}$ & -1.3214 & -0.6471 & -0.9696 & -1.6795 & -2.3619 & -1.2929 & -0.3760 & -2.0731 & -2.2946 & -1.7351 & -1.7674 & 2.4788 & 0.0106 & 1.9575 \\
$\mathrm{y}$ & -0.7768 & -0.9256 & -0.6982 & -1.6906 & -1.1517 & -1.6534 & -1.6752 & -0.0374 & -0.6665 & -1.1394 & -1.7696 & 1.7392 & 1.2392 & 1.8667 \\
$\mathrm{z}$ & -1.4703 & -0.9825 & -0.1957 & -1.2848 & -2.3504 & -1.0046 & -0.8595 & -0.6855 & -2.4252 & -2.0428 & -1.0916 & 0.6988 & 0.1998 & 1.0911 \\
$\mathrm{~d}$ & 0.5022 & 0.5064 & 0.5156 & 0.5274 & 0.5214 & 0.5127 & 0.5050 & 0.5172 & 0.5304 & 0.5573 & 0.5099 & 0.5305 & 0.5404 & 0.5592 \\
$\mathrm{t}$ & 4.3085 & 4.7590 & 2.5392 & 7.2077 & 4.6043 & 9.8650 & 5.2281 & 5.4614 & 8.4398 & 1.6075 & 7.5171 & 1.7118 & 9.0330 & 5.8479 \\
\hline
\end{tabular}
Fibre No:

\begin{tabular}{lllllllllllllllllll}
\multicolumn{2}{c}{29} & 30 & 31 & 32 & 33 & 34 & 35 & 36 & 37 & 38 & 39 & 40 & 41 & 42 \\
\hline $\mathrm{x}$ & 1.2962 & 0.9644 & 0.7007 & 0.0234 & 0.1382 & 0.1265 & 0.3758 & 1.7029 & 0.0041 & 1.4404 & 0.3063 & 1.5190 & 2.0953 & 2.0326 \\
$\mathrm{y}$ & 0.1671 & 1.5672 & 2.0398 & 0.2595 & 0.1766 & 1.1945 & 0.7149 & 0.8279 & 2.1405 & 1.8474 & 2.4334 & 0.3148 & 2.4356 & 2.2448 \\
$\mathrm{z}$ & 1.3601 & 1.6476 & 0.9298 & 1.3024 & 0.4007 & 1.1429 & 2.0842 & 0.1437 & 0.0734 & 1.6983 & 0.4707 & 1.3822 & 0.6475 & 0.6115 \\
$\mathrm{~d}$ & 0.5132 & 0.5251 & 0.5223 & 0.5331 & 0.5269 & 0.5024 & 0.5235 & 0.5584 & 0.5147 & 0.5044 & 0.5178 & 0.5545 & 0.5298 & 0.5313 \\
$\mathrm{t}$ & 2.1583 & 5.3866 & 3.1017 & 2.2069 & 2.9702 & 4.4649 & 5.7778 & 5.4239 & 4.8965 & 0.3559 & 6.1413 & 3.5692 & 4.2434 & 6.9486 \\
\hline
\end{tabular}
Fibre No:

\begin{tabular}{lllllllllll}
\multicolumn{2}{c}{43} & 44 & 45 & 46 & 47 & 48 & 49 & 50 \\
\hline $\mathrm{x}$ & 1.1296 & 0.2842 & 1.0798 & 0.3091 & 1.9236 & 1.2499 & 0.0909 & 1.5415 \\
$\mathrm{y}$ & 0.6591 & 1.3674 & 0.2063 & 2.4334 & 2.3351 & 1.8731 & 0.5764 & 0.9287 \\
$\mathrm{z}$ & 1.6638 & 0.0152 & 1.7185 & 0.0741 & 0.6254 & 1.6798 & 0.5542 & 0.5620 \\
$\mathrm{~d}$ & 0.5460 & 0.5389 & 0.5150 & 0.5504 & 0.5168 & 0.5218 & 0.5075 & 0.5320 \\
$\mathrm{t}$ & 2.0956 & 5.9936 & 8.3621 & 4.9424 & 7.6911 & 7.5677 & 6.5262 & 6.5301
\end{tabular}

Table A.4.7 Myopathy MUAP - diameter variability, results after 251 iterations. 
Results after 1011 iterations $\mathrm{x}, \mathrm{y}, \mathrm{z}, \mathrm{d}$ in $\mathrm{mm}, \mathrm{t}$ in $\mathrm{ms}$ (o_db1000.mat)

Fibre No:

\begin{tabular}{|c|c|c|c|c|c|c|c|c|c|c|c|c|c|c|}
\hline & 2 & 2 & 3 & 4 & 5 & 6 & 7 & 8 & 9 & 10 & 11 & 12 & 13 & 14 \\
\hline $\mathrm{X}$ & -1.0490 & .7814 & -1.7164 & -2.3804 & .7123 & -0.4060 & -2.1471 & -2.0724 & -0.2679 & -1.7428 & -1.5686 & -1.9184 & -1.9936 & -2.0 \\
\hline & -1.6837 & 0171 & -0.5879 & 56 & 9277 & 16 & -0.3239 & -0.3886 & $5-1$ & 84 & 53 & 51 & 2595 & -1 \\
\hline$Z$ & -1.6789 & -0.2301 & -0.5871 & -0.9610 & -1.9451 & -0.5362 & -0 & 37 & -1 & -1.6525 & -0.7799 & -1.3425 & -0.2933 & -1.8133 \\
\hline d & 0.7086 & 0.7955 & 1.2288 & 0.6867 & 0.6363 & 0.9562 & 1.1402 & 0.6973 & 0.7682 & 0.6763 & 1.0619 & 0.7796 & 1.3807 & 0.7166 \\
\hline & 8.3642 & 7.6400 & 3.8882 & 6.9217 & 2.8216 & 2.4405 & 1.7688 & 2.3360 & 7.9477 & 0.5644 & 1.1744 & 3.2967 & 8.6023 & 1.3533 \\
\hline
\end{tabular}
Fibre No:

\begin{tabular}{rrrrrrrrrrrrrrr}
\multicolumn{1}{l}{15} & 16 & 17 & 18 & \multicolumn{1}{c}{19} & \multicolumn{2}{c}{20} & 21 & 22 & 23 & 24 & 25 & 26 & 27 & 28 \\
\hline $\mathrm{x}$ & -1.3397 & -0.6694 & -1.0146 & -1.6871 & -2.3616 & -1.3041 & -0.3862 & -2.1731 & -2.2952 & -1.7397 & -1.7752 & 2.4726 & -0.0091 & 1.9514 \\
$\mathrm{y}$ & -0.7530 & -0.9002 & -0.7688 & -1.6529 & -1.1372 & -1.6150 & -1.6476 & -0.0492 & -0.6565 & -1.1179 & -1.7293 & 1.7275 & 1.2068 & 1.8532 \\
$\mathrm{z}$ & -1.4251 & -0.9556 & -0.2154 & -1.2562 & -2.3208 & -0.9813 & -0.8454 & -0.9029 & -2.3889 & -2.0042 & -1.0667 & 0.6941 & 0.1946 & 1.0832 \\
$\mathrm{~d}$ & 0.7969 & 0.8204 & 0.9679 & 0.7527 & 0.6711 & 0.7553 & 0.7183 & 1.4403 & 0.6970 & 0.7577 & 0.7378 & 0.6678 & 0.8405 & 0.7003 \\
$\mathrm{t}$ & 4.3085 & 4.7590 & 2.5392 & 7.2077 & 4.6043 & 9.8650 & 5.2281 & 5.4614 & 8.4398 & 1.6075 & 7.5171 & 1.7118 & 9.0330 & 5.8479 \\
\hline
\end{tabular}
Fibre No:

\begin{tabular}{cccccccccccccccccc}
\multicolumn{2}{r}{29} & 30 & 31 & 32 & 33 & 34 & 35 & 36 & 37 & 38 & 39 & 40 & 41 & 42 \\
\hline $\mathrm{x}$ & 1.2850 & 0.9589 & 0.6949 & 0.0051 & 0.1177 & 0.1149 & 0.3694 & 1.6849 & -0.0028 & 1.4366 & 0.3019 & 1.5071 & 2.0914 & 2.0279 \\
$\mathrm{y}$ & 0.1647 & 1.5547 & 2.0219 & 0.2532 & 0.1237 & 1.1749 & 0.7076 & 0.8057 & 2.1154 & 1.8376 & 2.4142 & 0.3101 & 2.4239 & 2.2321 \\
$\mathrm{z}$ & 1.3403 & 1.6345 & 0.9216 & 1.2708 & 0.2808 & 1.1241 & 2.0631 & 0.1398 & 0.0725 & 1.6894 & 0.4670 & 1.3615 & 0.6444 & 0.6080 \\
$\mathrm{~d}$ & 0.7161 & 0.6706 & 0.6749 & 0.8177 & 0.9496 & 0.7232 & 0.6868 & 0.8051 & 0.6899 & 0.6217 & 0.6579 & 0.7596 & 0.6428 & 0.6551 \\
$\mathrm{t}$ & 2.1583 & 5.3866 & 3.1017 & 2.2069 & 2.9702 & 4.4649 & 5.7778 & 5.4239 & 4.8965 & 0.3559 & 6.1413 & 3.5692 & 4.2434 & 6.9486 \\
\hline
\end{tabular}
Fibre No:

\begin{tabular}{lllllllllll}
\multicolumn{2}{c}{43} & 44 & 45 & 46 & 47 & 48 & 49 & 50 \\
\hline $\mathrm{x}$ & 1.1202 & 0.2678 & 1.0709 & 0.3038 & 1.9194 & 1.2458 & 0.0759 & 1.5275 \\
$\mathrm{y}$ & 0.6511 & 1.3369 & 0.2039 & 2.4108 & 2.3230 & 1.8620 & 0.5534 & 0.9108 \\
$\mathrm{z}$ & 1.6436 & 0.0149 & 1.6987 & 0.0734 & 0.6222 & 1.6698 & 0.5321 & 0.5512 \\
$\mathrm{~d}$ & 0.7317 & 0.8047 & 0.6964 & 0.7029 & 0.6345 & 0.6457 & 0.8484 & 0.7552 \\
$\mathrm{t}$ & 2.0956 & 5.9936 & 8.3621 & 4.9424 & 7.6911 & 7.5677 & 6.5262 & 6.5301
\end{tabular}

Table A.4.8 Myopathy MUAP - diameter variability, results after 1011 iterations. 
Results after 5066 iterations $\mathrm{x}, \mathrm{y}, \mathrm{z}, \mathrm{d}$ in $\mathrm{mm}, \mathrm{t}$ in $\mathrm{ms}$ (o_dn5000.mat)

Fibre No:

\begin{tabular}{lrrrrrrrrrrrrrrrrr} 
& \multicolumn{2}{c}{2} & 3 & 4 & 5 & 6 & 7 & 8 & 9 & 10 & 11 & 12 & 13 & 14 \\
\hline $\mathrm{x}$ & -1.0679 & -1.8046 & -1.7485 & -2.4284 & -0.7267 & -0.4612 & -2.0598 & -2.1112 & -0.2733 & -1.7712 & -1.6737 & -1.9276 & -2.0654 & -2.0732 \\
$\mathrm{y}$ & -1.7021 & -2.1259 & -0.6480 & -2.3679 & -1.9303 & -0.0346 & -0.5656 & -0.3949 & -0.5485 & -1.8800 & -0.5330 & -1.3747 & -0.2220 & -1.4103 \\
$\mathrm{z}$ & -1.6972 & -0.2425 & -0.6471 & -0.9742 & -1.9477 & -0.5871 & -1.6502 & -2.4737 & -1.5867 & -1.6717 & -0.7484 & -1.4473 & -0.2509 & -1.8716 \\
$\mathrm{~d}$ & 0.9590 & 1.0944 & 1.4460 & 1.0427 & 0.8499 & 1.0190 & 1.2933 & 1.0226 & 0.9650 & 0.9634 & 1.3219 & 1.0485 & 1.1494 & 1.0346 \\
$\mathrm{t}$ & 8.3642 & 7.6400 & 3.8882 & 6.9217 & 2.8216 & 2.4405 & 1.7688 & 2.3360 & 7.9477 & 0.5644 & 1.1744 & 3.2967 & 8.6023 & 1.3533 \\
\hline
\end{tabular}
Fibre No:

\begin{tabular}{lrrrrrrrrrrrrrrrr}
\multicolumn{1}{l}{15} & 16 & 17 & \multicolumn{2}{c}{18} & \multicolumn{1}{c}{19} & 20 & 21 & 22 & 23 & 24 & 25 & 26 & 27 & 28 \\
\hline $\mathrm{x}$ & -1.3317 & -0.6736 & -1.0297 & -1.7083 & -2.4059 & -1.3116 & -0.3932 & -2.3347 & -2.3418 & -1.7720 & -1.7946 & 2.4686 & -0.0120 & 1.9456 \\
$\mathrm{y}$ & -0.7860 & -0.9301 & -0.9528 & -1.7201 & -1.1478 & -1.6727 & -1.6737 & -0.1265 & -0.6680 & -1.1478 & -1.8086 & 1.7307 & 1.2592 & 1.8566 \\
$\mathrm{z}$ & -1.4877 & -0.9874 & -0.2670 & -1.3072 & -2.3424 & -1.0163 & -0.8588 & -2.3196 & -2.4305 & -2.0578 & -1.1156 & 0.6954 & 0.2030 & 1.0852 \\
$\mathrm{~d}$ & 1.0153 & 1.0094 & 1.1101 & 1.0347 & 1.0170 & 0.9957 & 0.9178 & 1.8817 & 1.0471 & 1.0687 & 1.0136 & 0.8039 & 1.0142 & 0.8530 \\
$\mathrm{t}$ & 4.3085 & 4.7590 & 2.5392 & 7.2077 & 4.6043 & 9.8650 & 5.2281 & 5.4614 & 8.4398 & 1.6075 & 7.5171 & 1.7118 & 9.0330 & 5.8479
\end{tabular}
Fibre No:

\begin{tabular}{|c|c|c|c|c|c|c|c|c|c|c|c|c|c|c|}
\hline 2 & & 30 & 31 & 32 & 33 & 34 & 35 & 36 & 37 & 38 & 39 & 40 & 41 & 42 \\
\hline $\mathrm{X}$ & 1.2843 & 0.9517 & 0.6871 & 0.0020 & 0.6288 & 0.1101 & 0.3608 & 1.6915 & -0.0120 & 1.4306 & 0.2924 & 1.5054 & 2.0859 & 2.0225 \\
\hline $\mathrm{y}$ & 0.1666 & 1.5592 & 2.0298 & 0.2621 & 0.1492 & 1.1935 & 0.7115 & 0.8029 & 2.1324 & 1.8390 & 2.4207 & 0.3132 & 2.4246 & 2.2342 \\
\hline z & 1.3555 & 1.6393 & 0.9252 & 1.3154 & 0.3385 & 1.1419 & 2.0744 & 0.1393 & 0.0731 & 1.6906 & 0.4682 & 1.3750 & 0.6446 & 0.6086 \\
\hline d & 0.8648 & 0.8372 & 0.8483 & 0.9937 & 0.9000 & 0.9002 & 0.8708 & 0.9595 & 0.8833 & 0.7677 & 0.8404 & 0.9175 & 0.7815 & 0.7975 \\
\hline $\mathrm{t}$ & 2.1583 & 5.3866 & 3.1017 & 2.2069 & 2.9702 & 4.4649 & 5.7778 & 5.4239 & 4.8965 & 0.3559 & 6.1413 & 3.5692 & 4.2434 & 6.9486 \\
\hline
\end{tabular}

\begin{tabular}{lllllllllll}
\multicolumn{1}{c}{43} & 44 & 45 & 46 & 47 & 48 & 49 & 50 \\
\hline $\mathrm{x}$ & 1.1151 & 0.2654 & 1.0666 & 0.2932 & 1.9141 & 1.2390 & 0.0820 & 1.5310 \\
$\mathrm{y}$ & 0.6560 & 1.3756 & 0.2055 & 2.4193 & 2.3246 & 1.8638 & 0.6530 & 0.9208 \\
$\mathrm{z}$ & 1.6560 & 0.0153 & 1.7118 & 0.0737 & 0.6226 & 1.6714 & 0.6278 & 0.5573 \\
$\mathrm{~d}$ & 0.8996 & 0.9785 & 0.8554 & 0.8973 & 0.7745 & 0.8014 & 0.9508 & 0.9020 \\
$\mathrm{t}$ & 2.0956 & 5.9936 & 8.3621 & 4.9424 & 7.6911 & 7.5677 & 6.5262 & 6.5301
\end{tabular}

Table A.4.9 Myopathy MUAP - diameter variability, results after 5066 iterations. 


\begin{tabular}{|c|c|c|c|c|c|c|c|c|c|c|c|c|c|}
\hline \multicolumn{14}{|c|}{ Simulated Values $\mathrm{x}, \mathrm{y}, \mathrm{z}, \mathrm{d}$ in $\mathrm{mm}, \mathrm{t}$ in $\mathrm{ms}$ (p_addmod.mat) } \\
\hline \multicolumn{14}{|c|}{ Fibre No: } \\
\hline 1 & 2 & 3 & 4 & 5 & 6 & 7 & 8 & 9 & 10 & 11 & 12 & 13 & 14 \\
\hline $\mathrm{X}-0.5802$ & -2.4173 & -2.4940 & -2.0385 & -2.0533 & -0.2413 & -1.2923 & -0.4800 & $0-2.3028$ & $8-0.0658$ & $8-1.4745$ & -2.0781 & 1.2270 & 1.3286 \\
\hline y -1.3413 & -2.1263 & -1.5988 & -1.3538 & -0.3885 & -1.2969 & -1.6863 & -1.6775 & $\begin{array}{ll}75 & -0.5541\end{array}$ & $1-1.7278$ & $8-2.3138$ & -2.2981 & 0.4035 & 1.7464 \\
\hline z $\quad-1.0765$ & -2.1688 & -1.1702 & -1.4320 & -0.1027 & -0.0560 & -1.8429 & -0.8647 & $.7-0.9503$ & $\begin{array}{ll}3 & -1.9059\end{array}$ & $9-2.3242$ & -1.5041 & 2.4403 & 1.6676 \\
\hline 0.5116 & 0.5226 & 0.5518 & 0.5409 & 0.5188 & 0.5074 & 0.5238 & 0.5520 & $\begin{array}{ll}0 & 0.5103\end{array}$ & $\begin{array}{ll}3 & 0.5256\end{array}$ & $6 \quad 0.5083$ & 0.5269 & 0.5089 & 0.5483 \\
\hline 9.8732 & 1.4839 & 6.9077 & 9.4525 & 1.1002 & 28.5688 & 0.2280 & 1.4071 & $1 \quad 1.6731$ & 0.7732 & 5.0597 & 5.1934 & 2.7325 & 5.4874 \\
\hline \multicolumn{14}{|l|}{ Fibre No: } \\
\hline $\begin{array}{ll}\mathrm{X} & 1.5268\end{array}$ & 1.1033 & 0.1290 & 0.2680 & 2.3381 & 1.7425 & 1.3355 & 1.3262 & 1.9575 & 0.5757 & 0.0120 & & & \\
\hline y 1.6699 & 0.3780 & 1.3052 & 0.9574 & 1.9301 & 1.0188 & 0.6916 & 2.3456 & 2.3006 & 0.3592 & 2.3398 & & & \\
\hline Z $\quad 1.2378$ & 1.2557 & 0.8016 & 1.3796 & 1.2829 & 1.2171 & 1.5138 & 0.5885 & 1.7037 & 2.2488 & 0.6884 & & & \\
\hline d $\quad 0.5086$ & 0.5278 & 0.5558 & 0.5117 & 0.5016 & 0.5024 & 0.5264 & 0.5104 & 0.5385 & 0.5298 & 0.5431 & & & \\
\hline t $\quad 5.8889$ & 0.3166 & 2.4514 & 6.1111 & 6.5013 & 7.1045 & 7.0337 & 5.9748 & 5.2741 & 6.5151 & 2.7893 & & & \\
\hline
\end{tabular}

Table A.4.10 Myopathy MUAP - reinnervation of 25 fibres in both planes of the MU, simulated values. 
Initial values $\mathrm{x}, \mathrm{y}, \mathrm{z}, \mathrm{d}$ in $\mathrm{mm}, \mathrm{t}$ in $\mathrm{ms}$ (zren1.mat)

\begin{tabular}{|c|c|c|c|c|c|c|c|c|c|c|c|c|c|c|}
\hline \\
\hline \multicolumn{2}{|c|}{$\begin{array}{l}\text { Fibre No: } \\
1\end{array}$} & 2 & 3 & 4 & 5 & 6 & 7 & 8 & 9 & 10 & 11 & 12 & 13 & 14 \\
\hline $\mathrm{X}$ & -0.5474 & -0.9588 & -1.3243 & -1.0437 & -1.3173 & -2.2758 & -0.8206 & -0.6 & -0.1817 & -1.9162 & -0.4163 & -2.2616 & -1.2349 & -1.2518 \\
\hline $\mathrm{y}$ & -0.1176 & -1.2985 & -1.6779 & -1.7169 & -0.2299 & -1.9055 & -1.5816 & -2.4564 & -1.5791 & -1.1943 & -1.2163 & -1.2613 & -0.6654 & -0.9604 \\
\hline $\mathrm{z}$ & -1.6972 & -2.0774 & -0.0192 & -1.4724 & -1.6348 & -0.6561 & -1.8910 & -1.8067 & -2.2118 & -0.5944 & -2.2441 & -1.2907 & -0.2268 & -0.6927 \\
\hline d & 0.5215 & 0.5559 & 0.5140 & 0.5517 & 0.5101 & 0.5543 & 0.5589 & 0.5304 & 0.5273 & 0.5270 & 0.5491 & 0.5217 & 0.5537 & 0.5497 \\
\hline & 9.3469 & 0.5346 & 0.6684 & 8.4617 & 7.0119 & 7.3608 & 3.6534 & 6.5152 & 4.3641 & 3.5926 & 0.6056 & 9.8664 & 0.7375 & 5.2975 \\
\hline
\end{tabular}
Fibre No:

\begin{tabular}{lrllllllllllllllll}
\multicolumn{1}{l}{15} & 16 & 17 & \multicolumn{2}{c}{18} & 19 & 20 & 21 & 22 & 23 & 24 & 25 & 26 & 27 & 28 \\
\hline $\mathrm{x}$ & -1.1611 & -2.0695 & -1.5739 & -0.5830 & -2.1150 & -1.3433 & -1.4291 & -2.3884 & -2.1051 & -0.2275 & -1.7745 & 2.2175 & 0.9693 & 1.4753 \\
$\mathrm{y}$ & -2.3524 & -0.3134 & -1.8406 & -0.7658 & -1.0302 & -1.1698 & -2.0060 & -1.8707 & -0.3994 & -0.6865 & -2.3447 & 1.6301 & 1.2494 & 2.3885 \\
$\mathrm{z}$ & -0.1252 & -0.0397 & -1.8135 & -0.8775 & -2.1038 & -0.7180 & -0.0826 & -1.3865 & -0.5319 & -0.0075 & -0.5998 & 0.3758 & 0.3688 & 1.3904 \\
$\mathrm{~d}$ & 0.5314 & 0.5226 & 0.5599 & 0.5016 & 0.5277 & 0.5386 & 0.5041 & 0.5469 & 0.5258 & 0.5103 & 0.5383 & 0.5218 & 0.5105 & 0.5422 \\
$\mathrm{t}$ & 7.7020 & 8.6825 & 8.8857 & 5.9111 & 4.1539 & 1.5372 & 4.9848 & 6.2485 & 1.3043 & 0.2688 & 3.1754 & 3.8581 & 8.4558 & 9.8331 \\
\hline
\end{tabular}
Fibre No:

\begin{tabular}{|c|c|c|c|c|c|c|c|c|c|c|c|c|c|c|}
\hline 2 & & 30 & 31 & 32 & 33 & 34 & 35 & 36 & 37 & 38 & 39 & 40 & 41 & 42 \\
\hline $\mathrm{x}$ & 1.0219 & 1.1601 & 1.5732 & 0.6196 & 2.2542 & 0.3280 & 0.9133 & 0.8738 & 0.5381 & 1.1782 & 1.1556 & 1.7225 & 0.7233 & 1.4418 \\
\hline $\mathrm{y}$ & 0.3545 & 2.4027 & 0.3168 & 1.1911 & 1.0662 & 2.2141 & 0.6326 & 1.1308 & 1.6990 & 1.2649 & 2.3784 & 1.7555 & 1.3436 & 2.1914 \\
\hline $\mathrm{z}$ & 1.4122 & 0.3151 & 1.6281 & 0.9733 & 0.3551 & 0.2304 & 0.3378 & 2.0224 & 2.2723 & 1.5010 & 1.5818 & 2.4679 & 1.2861 & 1.1001 \\
\hline d & 0.5297 & 0.5360 & 0.5146 & 0.5356 & 0.5537 & 0.5405 & 0.5340 & 0.5518 & 0.5300 & 0.5381 & 0.5073 & 0.5545 & 0.5476 & 0.5276 \\
\hline $\mathrm{t}$ & 4.8851 & 3.1925 & 8.0307 & 0.2838 & 4.1031 & 0.7106 & 4.5531 & 6.5165 & 8.6086 & 7.5584 & 8.2470 & 8.5127 & 4.1403 & 8.6926 \\
\hline
\end{tabular}

\begin{tabular}{|c|c|c|c|c|c|c|c|c|c|c|c|c|c|c|}
\hline & & 44 & 45 & 46 & 47 & 48 & 49 & 50 & 1 & & 53 & 54 & 55 & 56 \\
\hline & 1.7 & & & & & & & & & & & & & \\
\hline & 2.0018 & 3125 & 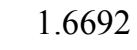 & 2.2250 & 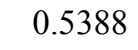 & 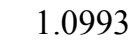 & 8 & 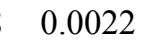 & -1 & & 46 & -2 & 22 & \\
\hline & 1.7663 & 1.1583 & & 1 & 1 & & 0 & & & & -0 & -0 & -1 & \\
\hline & 0.5290 & 0.5522 & 1 & 0 . & 0 . & 0 & 77 & 0. & 0 & 02 & 03 & 277 & 93 & 0. \\
\hline & 0.1909 & .1342 & 9.1663 & 4.5035 & 5.1466 & 3.6516 & 6.1635 & 3.1918 & 8.3642 & 7.6400 & 3.8882 & 6.9217 & 2.8216 & 2.4405 \\
\hline
\end{tabular}




\begin{tabular}{|c|c|c|c|c|c|c|c|c|c|c|c|c|c|c|}
\hline \multicolumn{15}{|c|}{ Fibre No: } \\
\hline & 57 & 58 & 59 & 60 & 61 & 62 & 63 & 64 & 65 & 66 & 67 & 68 & 69 & 70 \\
\hline $\mathrm{X}$ & -2.0493 & -2.0709 & -0.2541 & -1.7406 & -1.4955 & -1.9064 & 1.7575 & 2.0332 & 1.3214 & 0.6471 & 0.9696 & 1.6795 & 2.3619 & 1.2929 \\
\hline $\mathrm{y}$ & -0.3411 & -0.3943 & -0.5485 & -1.8824 & -0.5675 & -1.3153 & 0.3576 & 1.3921 & 0.7768 & 0.9256 & 0.6982 & 1.6906 & 1.1517 & 1.6534 \\
\hline $\mathrm{Z}$ & -0.9954 & -2.4698 & -1.5868 & -1.6738 & -0.7969 & -1.3848 & 0.4042 & 1.8475 & 1.4703 & 0.9825 & 0.1957 & 1.2848 & 2.3504 & 1.0046 \\
\hline d & 0.5121 & 0.5291 & 0.5235 & 0.5160 & 0.5240 & 0.5106 & 0.5018 & 0.5221 & 0.5022 & 0.5064 & 0.5156 & 0.5274 & 0.5214 & 0.5127 \\
\hline$\underline{t}$ & 1.7688 & 2.3360 & 7.9477 & 0.5644 & 1.1744 & 3.2967 & 8.6023 & 1.3533 & 4.3085 & 4.7590 & 2.5392 & 7.2077 & 4.6043 & 9.8650 \\
\hline \multicolumn{15}{|c|}{ Fibre No: } \\
\hline & 71 & 72 & 73 & 74 & 75 & & & & & & & & & \\
\hline$x$ & 0.3760 & 2.0731 & 2.2946 & 1.7351 & 1.7674 & & & & & & & & & \\
\hline $\mathrm{y}$ & 1.6752 & 0.0374 & 0.6665 & 1.1394 & 1.7696 & & & & & & & & & \\
\hline z & 0.8595 & 0.6855 & 2.4252 & 2.0428 & 1.0916 & & & & & & & & & \\
\hline d & 0.5050 & 0.5172 & 0.5304 & 0.5573 & 0.5099 & & & & & & & & & \\
\hline $\mathrm{t}$ & 5.2281 & 5.4614 & 8.4398 & 1.6075 & 7.5171 & & & & & & & & & \\
\hline
\end{tabular}

Table A.4.11 Myopathy MUAP - reinnervation of 25 fibres in both planes of the MU, initial values. 


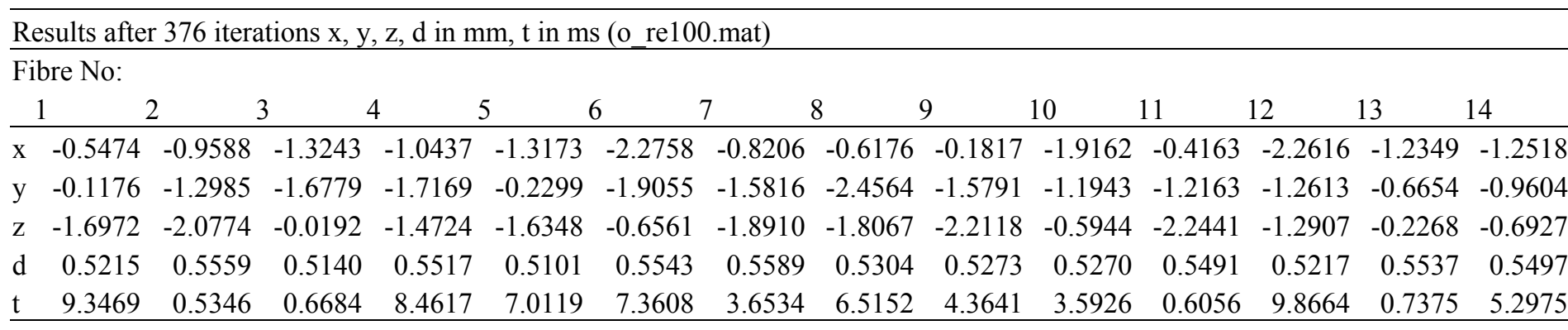
Fibre No:

\begin{tabular}{|c|c|c|c|c|c|c|c|c|c|c|c|c|c|c|}
\hline 1 & 5 & 16 & 17 & 18 & 19 & 20 & 21 & 22 & 23 & 24 & 25 & 26 & 27 & 28 \\
\hline $\mathrm{x}$ & -1.1611 & -2.0695 & -1.5739 & -0.5830 & -2.1150 & -1.3433 & -1.4291 & -2.3884 & -2.1051 & -0.2275 & -1.7745 & 2.2175 & 0.9693 & 1.4753 \\
\hline y & -2.3524 & -0.3134 & -1.8406 & -0.7658 & -1.0302 & -1.1698 & -2.0060 & -1.8707 & -0.3994 & -0.6865 & -2.3447 & $\begin{array}{ll}7 & 1.6301\end{array}$ & 1.2494 & 2.3885 \\
\hline z & -0.1252 & -0.0397 & -1.8135 & -0.8775 & -2.1038 & -0.7180 & -0.0826 & -1.3865 & -0.5319 & -0.0075 & -0.5998 & $\begin{array}{ll}3 & 0.3758\end{array}$ & 0.3688 & 1.3904 \\
\hline d & 0.5314 & 0.5226 & 0.5599 & 0.5016 & 0.5277 & 0.5386 & 0.5041 & 0.5469 & 0.5258 & 0.5103 & 0.5383 & $\begin{array}{ll}3 & 0.5218\end{array}$ & 0.5105 & 0.5422 \\
\hline $\mathrm{t}$ & 7.7020 & 8.6825 & 8.8857 & 5.9111 & 4.1539 & 1.5372 & 4.9848 & $8 \quad 6.2485$ & 1.3043 & 0.2688 & 3.1754 & $\begin{array}{l}4 \quad 3.8581 \\
\end{array}$ & 8.4558 & 9.8331 \\
\hline
\end{tabular}
Fibre No:

\begin{tabular}{lllllllllllllllllll}
\multicolumn{2}{c}{29} & 30 & 31 & 32 & 33 & 34 & 35 & 36 & 37 & 38 & 39 & 40 & 41 & 42 \\
\hline $\mathrm{x}$ & 1.0219 & 1.1601 & 1.5732 & 0.6196 & 2.2542 & 0.3280 & 0.9133 & 0.8738 & 0.5381 & 1.1782 & 1.1556 & 1.7225 & 0.7233 & 1.4418 \\
$\mathrm{y}$ & 0.3545 & 2.4027 & 0.3168 & 1.1911 & 1.0662 & 2.2141 & 0.6326 & 1.1308 & 1.6990 & 1.2649 & 2.3784 & 1.7555 & 1.3436 & 2.1914 \\
$\mathrm{z}$ & 1.4122 & 0.3151 & 1.6281 & 0.9733 & 0.3551 & 0.2304 & 0.3378 & 2.0224 & 2.2723 & 1.5010 & 1.5818 & 2.4679 & 1.2861 & 1.1001 \\
$\mathrm{~d}$ & 0.5297 & 0.5360 & 0.5146 & 0.5356 & 0.5537 & 0.5405 & 0.5340 & 0.5518 & 0.5300 & 0.5381 & 0.5073 & 0.5545 & 0.5476 & 0.5276 \\
$\mathrm{t}$ & 4.8851 & 3.1925 & 8.0307 & 0.2838 & 4.1031 & 0.7106 & 4.5531 & 6.5165 & 8.6086 & 7.5584 & 8.2470 & 8.5127 & 4.1403 & 8.6926 \\
\hline
\end{tabular}
Fibre No:

\begin{tabular}{lrllllllllllllllll}
\multicolumn{1}{r}{43} & 44 & 45 & 46 & 47 & 48 & 49 & 50 & 51 & 52 & 53 & 54 & 55 & 56 \\
$\mathrm{x}$ & 1.7891 & 2.2151 & 1.2224 & 2.1647 & 2.4734 & 2.2038 & 0.5288 & 0.0015 & -1.0443 & -1.7723 & -1.5739 & -2.3804 & -0.7101 & -0.4849 \\
$\mathrm{y}$ & 2.0018 & 1.3125 & 1.6692 & 2.2250 & 0.5388 & 1.0993 & 2.4978 & 0.0022 & -1.7062 & -2.0718 & -0.5346 & -2.3689 & -1.9422 & -0.0283 \\
$\mathrm{z}$ & 1.7663 & 1.1583 & 1.7051 & 1.3599 & 1.1151 & 1.1688 & 0.3840 & 1.9334 & -1.7014 & -0.2364 & -0.5339 & -0.9746 & -1.9597 & -0.4796 \\
$\mathrm{~d}$ & 0.5290 & 0.5522 & 0.5361 & 0.5433 & 0.5221 & 0.5368 & 0.5157 & 0.5273 & 0.5354 & 0.5502 & 0.5503 & 0.5277 & 0.5093 & 0.5580 \\
$\mathrm{t}$ & 0.1909 & 7.1342 & 9.1663 & 4.5035 & 5.1466 & 3.6516 & 6.1635 & 3.1918 & 8.3642 & 7.6400 & 3.8882 & 6.9217 & 2.8216 & 2.4405
\end{tabular}




\begin{tabular}{|c|c|c|c|c|c|c|c|c|c|c|c|c|c|c|}
\hline \multicolumn{15}{|c|}{ Fibre No: } \\
\hline & 57 & 58 & 59 & 60 & 61 & 62 & 63 & 64 & 65 & 66 & 67 & 68 & 69 & 70 \\
\hline $\mathrm{x}$ & -2.0493 & -2.0709 & -0.2541 & -1.7406 & -1.4955 & -1.9064 & 1.7575 & 2.0332 & 1.3214 & 0.6471 & 0.9696 & 1.6795 & 2.3619 & 1.2929 \\
\hline $\mathrm{y}$ & -0.3411 & -0.3943 & -0.5485 & -1.8824 & -0.5675 & -1.3153 & 0.3576 & 1.3921 & 0.7768 & 0.9256 & 0.6982 & 1.6906 & 1.1517 & 1.6534 \\
\hline $\mathrm{Z}$ & -0.9954 & -2.4698 & -1.5868 & -1.6738 & -0.7969 & -1.3848 & 0.4042 & 1.8475 & 1.4703 & 0.9825 & 0.1957 & 1.2848 & 2.3504 & 1.0046 \\
\hline d & 0.5121 & 0.5291 & 0.5235 & 0.5160 & 0.5240 & 0.5106 & 0.5018 & 0.5221 & 0.5022 & 0.5064 & 0.5156 & 0.5274 & 0.5214 & 0.5127 \\
\hline$\underline{t}$ & 1.7688 & 2.3360 & 7.9477 & 0.5644 & 1.1744 & 3.2967 & 8.6023 & 1.3533 & 4.3085 & 4.7590 & 2.5392 & 7.2077 & 4.6043 & 9.8650 \\
\hline \multicolumn{15}{|c|}{ Fibre No: } \\
\hline & 71 & 72 & 73 & 74 & 75 & & & & & & & & & \\
\hline$x$ & 0.3760 & 2.0731 & 2.2946 & 1.7351 & 1.7674 & & & & & & & & & \\
\hline$y$ & 1.6752 & 0.0374 & 0.6665 & 1.1394 & 1.7696 & & & & & & & & & \\
\hline $\mathrm{z}$ & 0.8595 & 0.6855 & 2.4252 & 2.0428 & 1.0916 & & & & & & & & & \\
\hline d & 0.5050 & 0.5172 & 0.5304 & 0.5573 & 0.5099 & & & & & & & & & \\
\hline $\mathrm{t}$ & 5.2281 & 5.4614 & 8.4398 & 1.6075 & 7.5171 & & & & & & & & & \\
\hline
\end{tabular}

Table A.4.12 Myopathy MUAP - reinnervation of 25 fibres in both planes of the MU, results after 376 iterations. 


\begin{tabular}{|c|c|c|c|c|c|c|c|c|c|c|c|c|c|c|}
\hline \multicolumn{15}{|c|}{ sults after 1132 iterations. $x, y, z, d$ in $\mathrm{mm}, \mathrm{t}$ in $\mathrm{ms}$ (o_re 1000 mat) } \\
\hline \multicolumn{15}{|c|}{ bre No: } \\
\hline & L & 2 & 3 & 4 & 5 & 6 & 7 & 8 & 9 & 10 & 11 & 12 & 13 & 14 \\
\hline & -0.5510 & -0.9609 & -1.3292 & -1.0464 & -1.3223 & -2.2799 & -0.8227 & -0.6186 & -0.1829 & -1.9255 & -0.4179 & -2.2664 & -1.2628 & -1.26 \\
\hline y & -0.1180 & -1.2988 & -1.6801 & -1.7175 & -0.2302 & -1.9015 & -1.5821 & -2.4565 & -1.5797 & -1.1928 & -1.2169 & -1.2583 & -0.6970 & -0.9691 \\
\hline $\mathrm{Z}$ & -1.7033 & -2.0778 & -0.0193 & -1.4729 & -1.6372 & -0.6548 & -1.8916 & -1.8067 & -2.2126 & -0.5937 & -2.2452 & -1.2877 & -0.2376 & -0.6990 \\
\hline 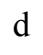 & 0.5143 & 0.5606 & 0.5194 & 0.5567 & 0.5151 & 0.5756 & 0.5627 & 0.5336 & 0.5280 & 0.5474 & 0.5504 & 0.5447 & 0.5113 & 0.5428 \\
\hline $\mathrm{t}$ & 9.3469 & 0.5346 & 0.6684 & 8.4617 & 7.0119 & 7.3608 & 3.6534 & 6.5152 & 4.3641 & 3.5926 & 0.6056 & 9.8664 & 0.7375 & 5.2975 \\
\hline
\end{tabular}
Fibre No:

\begin{tabular}{|c|c|c|c|c|c|c|c|c|c|c|c|c|c|c|}
\hline & 15 & 16 & 17 & 18 & 19 & 20 & 21 & 22 & 23 & 24 & 25 & 26 & 27 & 28 \\
\hline $\mathbf{y}$ & -1.1634 & -2.0839 & -1.5759 & -0.5895 & -2.1174 & -1.3515 & -1.4323 & -2.3910 & -2.1320 & -0.23 & -1.7768 & 2.2180 & 0.9695 & 1.4747 \\
\hline & -2.3526 & -0.2979 & -1.8398 & -0.7774 & -1.0291 & -1.1745 & -2.0061 & -1.8678 & -0.3887 & -0.7500 & -2.3433 & 360 & 28 & 2.3 \\
\hline & -0.1252 & -0.0377 & -1.8128 & -0.8908 & -2.1015 & -0.7209 & -0.0826 & -1.3843 & -0.5176 & $5-0.0082$ & -0.5994 & 0.3772 & 0.3728 & 4 \\
\hline $\mathrm{a}$ & 0.5374 & 0.6009 & 0.5692 & 1731 & 422 & 405 & 23 & 0 & 739 & 1053 & 0.5495 & 013 & 749 & 0 \\
\hline & 7.7020 & 8.6825 & 8.8857 & 5.9111 & 4.1539 & 1.5372 & 4.9848 & 6.2485 & 1.3043 & 0.2688 & 3.1754 & 3.8581 & 8.4558 & 9.8331 \\
\hline
\end{tabular}
Fibre No:

\begin{tabular}{llllllllllllllllll}
\multicolumn{2}{c}{29} & 30 & 31 & 32 & 33 & 34 & 35 & 36 & 37 & 38 & 39 & 40 & 41 & 42 \\
\hline $\mathrm{x}$ & 1.0217 & 1.1593 & 1.5733 & 0.6183 & 2.2572 & 0.3265 & 0.9214 & 0.8728 & 0.5372 & 1.1774 & 1.1550 & 1.7220 & 0.7220 & 1.4412 \\
$\mathrm{y}$ & 0.3574 & 2.4057 & 0.3182 & 1.1998 & 1.0765 & 2.2177 & 0.6682 & 1.1325 & 1.6998 & 1.2686 & 2.3796 & 1.7564 & 1.3486 & 2.1939 \\
$\mathrm{z}$ & 1.4237 & 0.3155 & 1.6353 & 0.9804 & 0.3585 & 0.2308 & 0.3568 & 2.0255 & 2.2734 & 1.5053 & 1.5827 & 2.4691 & 1.2909 & 1.1013 \\
$\mathrm{~d}$ & 0.5006 & 0.5292 & 0.4932 & 0.5109 & 0.5150 & 0.5346 & 0.4410 & 0.5444 & 0.5284 & 0.5240 & 0.5042 & 0.5510 & 0.5325 & 0.5204 \\
$\mathrm{t}$ & 4.8851 & 3.1925 & 8.0307 & 0.2838 & 4.1031 & 0.7106 & 4.5531 & 6.5165 & 8.6086 & 7.5584 & 8.2470 & 8.5127 & 4.1403 & 8.6926 \\
\hline
\end{tabular}
Fibre No:

\begin{tabular}{lrlllllllllllllll}
\multicolumn{1}{r}{43} & 44 & 45 & 46 & 47 & 48 & 49 & 50 & 51 & 52 & 53 & 54 & 55 & 56 \\
\hline $\mathrm{x}$ & 1.7887 & 2.2155 & 1.2216 & 2.1644 & 2.4754 & 2.2045 & 0.5278 & -0.0007 & -1.0464 & -1.7759 & -1.6001 & -2.3822 & -0.7113 & -0.5060 \\
$\mathrm{y}$ & 2.0034 & 1.3171 & 1.6714 & 2.2271 & 0.5422 & 1.1040 & 2.4999 & 0.0022 & -1.7065 & -2.0702 & -0.5422 & -2.3663 & -1.9424 & -0.0356 \\
$\mathrm{z}$ & 1.7678 & 1.1624 & 1.7074 & 1.3611 & 1.1221 & 1.1738 & 0.3843 & 1.9385 & -1.7017 & -0.2362 & -0.5414 & -0.9736 & -1.9599 & -0.6034 \\
$\mathrm{~d}$ & 0.5231 & 0.5323 & 0.5286 & 0.5362 & 0.4913 & 0.5140 & 0.5122 & 0.5191 & 0.5405 & 0.5636 & 0.5450 & 0.5422 & 0.5126 & 0.3871 \\
$\mathrm{t}$ & 0.1909 & 7.1342 & 9.1663 & 4.5035 & 5.1466 & 3.6516 & 6.1635 & 3.1918 & 8.3642 & 7.6400 & 3.8882 & 6.9217 & 2.8216 & 2.4405 \\
\hline
\end{tabular}




\begin{tabular}{|c|c|c|c|c|c|c|c|c|c|c|c|c|c|c|}
\hline \multicolumn{15}{|c|}{ Fibre No: } \\
\hline & 57 & 58 & 59 & 60 & 61 & 62 & 63 & 64 & 65 & 66 & 67 & 68 & 69 & 70 \\
\hline $\mathrm{x}$ & -2.0629 & -2.0729 & -0.2572 & -1.7425 & -1.5109 & -1.9104 & 1.7674 & 2.0329 & 1.3212 & 0.6463 & 0.9771 & 1.6791 & 2.3616 & 1.2923 \\
\hline $\mathrm{y}$ & -0.3396 & -0.3940 & -0.5510 & -1.8814 & -0.5729 & -1.3140 & 0.3692 & 1.3940 & 0.7803 & 0.9351 & 0.7324 & 1.6939 & 1.1527 & 1.6579 \\
\hline $\mathrm{Z}$ & -0.9910 & -2.4677 & -1.5939 & -1.6730 & -0.8045 & -1.3834 & 0.4173 & 1.8500 & 1.4768 & 0.9926 & 0.2052 & 1.2874 & 2.3523 & 1.0073 \\
\hline d & 0.5407 & 0.5420 & 0.5119 & 0.5260 & 0.5214 & 0.5259 & 0.4266 & 0.5124 & 0.4815 & 0.4741 & 0.4285 & 0.5155 & 0.5145 & 0.4984 \\
\hline$\underline{t}$ & 1.7688 & 2.3360 & 7.9477 & 0.5644 & 1.1744 & 3.2967 & 8.6023 & 1.3533 & 4.3085 & 4.7590 & 2.5392 & 7.2077 & 4.6043 & 9.8650 \\
\hline \multicolumn{15}{|c|}{ Fibre No: } \\
\hline & 71 & 72 & 73 & 74 & 75 & & & & & & & & & \\
\hline$x$ & 0.3744 & 2.0798 & 2.2944 & 1.7346 & 1.7671 & & & & & & & & & \\
\hline$y$ & 1.6802 & 0.0381 & 0.6672 & 1.1411 & 1.7730 & & & & & & & & & \\
\hline $\mathrm{z}$ & 0.8621 & 0.6988 & 2.4276 & 2.0459 & 1.0937 & & & & & & & & & \\
\hline d & 0.4935 & 0.4587 & 0.5225 & 0.5478 & 0.4976 & & & & & & & & & \\
\hline $\mathrm{t}$ & 5.2281 & 5.4614 & 8.4398 & 1.6075 & 7.5171 & & & & & & & & & \\
\hline
\end{tabular}

Table A.4.13 Myopathy MUAP - reinnervation of 25 fibres in both planes of the MU, results after 1132 iterations. 


\begin{tabular}{|c|c|c|c|c|c|c|c|c|c|c|c|c|c|c|}
\hline \multicolumn{15}{|c|}{ esults after 5294 iterations. $\mathrm{x}, \mathrm{y}, \mathrm{z}, \mathrm{d}$ in $\mathrm{mm}$. $\mathrm{t}$ in $\mathrm{ms}$ (o_re 5000 mat) } \\
\hline \multicolumn{15}{|c|}{ bre No: } \\
\hline & L & 2 & 3 & 4 & 5 & 6 & 7 & 8 & 9 & 10 & 11 & 12 & 13 & 14 \\
\hline A & -0.5506 & -0.9627 & -1.3287 & -1.0478 & -1.3217 & -2.2821 & -0.8243 & -0.6202 & -0.1842 & -1.9230 & -0.4193 & -2.2669 & -1.2787 & -1.25 \\
\hline y & -0.1184 & -1.3009 & -1.6890 & -1.7215 & -0.2315 & -1.9145 & -1.5845 & -2.4572 & -1.5808 & -1.2074 & -1.2182 & -1.2688 & -0.7414 & -0.98 \\
\hline $\mathrm{Z}$ & -1.7083 & -2.0812 & -0.0194 & -1.4763 & -1.6460 & -0.6592 & -1.8945 & -1.8073 & -2.2142 & -0.6009 & -2.2477 & -1.2984 & -0.2528 & -0.7084 \\
\hline 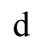 & 0.5198 & 0.5818 & 0.5227 & 0.5752 & 0.5174 & 0.5953 & 0.5834 & 0.5564 & 0.5464 & 0.5311 & 0.5694 & 0.5532 & 0.4489 & 0.52 \\
\hline & 9.3469 & 0.5346 & 0.6684 & 8.4617 & 7.0119 & 7.3608 & 3.6534 & 6.5152 & 4.3641 & 3.5926 & 0.6056 & 9.8664 & 0.7375 & 5.297 \\
\hline
\end{tabular}
Fibre No:

\begin{tabular}{rrrrrrrrrrrrrrrr}
\multicolumn{1}{l}{15} & 16 & 17 & 18 & 19 & 20 & 21 & 22 & 23 & 24 & 25 & 26 & 27 & 28 \\
\hline $\mathrm{x}$ & -1.1650 & -2.0678 & -1.5785 & -0.5847 & -2.1203 & -1.3494 & -1.4332 & -2.3948 & -2.0676 & -0.1810 & -1.7794 & 2.2175 & 0.9676 & 1.4739 \\
$\mathrm{y}$ & -2.3572 & -0.3328 & -1.8426 & -0.7814 & -1.0321 & -1.1853 & -2.0131 & -1.8746 & -0.3747 & -0.6727 & -2.3485 & 1.6397 & 1.2639 & 2.3915 \\
$\mathrm{z}$ & -0.1255 & -0.0421 & -1.8155 & -0.8954 & -2.1077 & -0.7275 & -0.0829 & -1.3894 & -0.4989 & -0.0073 & -0.6007 & 0.3780 & 0.3731 & 1.3921 \\
$\mathrm{~d}$ & 0.5576 & 0.6784 & 0.5974 & 0.4673 & 0.5702 & 0.5316 & 0.5256 & 0.5969 & 0.5122 & 0.4516 & 0.5760 & 0.5017 & 0.4762 & 0.5502 \\
$\mathrm{t}$ & 7.7020 & 8.6825 & 8.8857 & 5.9111 & 4.1539 & 1.5372 & 4.9848 & 6.2485 & 1.3043 & 0.2688 & 3.1754 & 3.8581 & 8.4558 & 9.8331 \\
\hline
\end{tabular}
Fibre No:

\begin{tabular}{llllllllllllllllll}
\multicolumn{2}{r}{29} & 30 & 31 & 32 & 33 & 34 & 35 & 36 & 37 & 38 & 39 & 40 & 41 & 42 \\
\hline $\mathrm{x}$ & 1.0204 & 1.1585 & 1.5724 & 0.6177 & 2.2568 & 0.3256 & 0.9016 & 0.8719 & 0.5362 & 1.1767 & 1.1542 & 1.7212 & 0.7213 & 1.4405 \\
$\mathrm{y}$ & 0.3579 & 2.4077 & 0.3188 & 1.2011 & 1.0866 & 2.2205 & 0.6777 & 1.1337 & 1.7006 & 1.2703 & 2.3806 & 1.7570 & 1.3506 & 2.1956 \\
$\mathrm{z}$ & 1.4258 & 0.3157 & 1.6384 & 0.9814 & 0.3618 & 0.2311 & 0.3618 & 2.0276 & 2.2745 & 1.5074 & 1.5833 & 2.4700 & 1.2929 & 1.1022 \\
$\mathrm{~d}$ & 0.5026 & 0.5403 & 0.4952 & 0.5151 & 0.5016 & 0.5467 & 0.4404 & 0.5559 & 0.5444 & 0.5308 & 0.5174 & 0.5644 & 0.5396 & 0.5308 \\
$\mathrm{t}$ & 4.8851 & 3.1925 & 8.0307 & 0.2838 & 4.1031 & 0.7106 & 4.5531 & 6.5165 & 8.6086 & 7.5584 & 8.2470 & 8.5127 & 4.1403 & 8.6926 \\
\hline
\end{tabular}
Fibre No:

\begin{tabular}{lrlllllllllllllll}
\multicolumn{1}{r}{43} & 44 & 45 & 46 & 47 & 48 & 49 & 50 & 51 & 52 & 53 & 54 & 55 & 56 \\
\hline $\mathrm{x}$ & 1.7880 & 2.2149 & 1.2208 & 2.1637 & 2.4753 & 2.2039 & 0.5269 & -0.0011 & -1.0481 & -1.7776 & -1.6079 & -2.3860 & -0.7128 & -0.3824 \\
$\mathrm{y}$ & 2.0045 & 1.3200 & 1.6729 & 2.2285 & 0.5454 & 1.1070 & 2.5018 & 0.0022 & -1.7094 & -2.0795 & -0.5359 & -2.3704 & -1.9437 & -0.0374 \\
$\mathrm{z}$ & 1.7687 & 1.1650 & 1.7089 & 1.3620 & 1.1287 & 1.1770 & 0.3846 & 1.9419 & -1.7045 & -0.2372 & -0.5351 & -0.9753 & -1.9612 & -0.6332 \\
$\mathrm{~d}$ & 0.5340 & 0.5339 & 0.5392 & 0.5460 & 0.4821 & 0.5134 & 0.5258 & 0.5282 & 0.5610 & 0.5822 & 0.5042 & 0.5777 & 0.5336 & 0.3618 \\
$\mathrm{t}$ & 0.1909 & 7.1342 & 9.1663 & 4.5035 & 5.1466 & 3.6516 & 6.1635 & 3.1918 & 8.3642 & 7.6400 & 3.8882 & 6.9217 & 2.8216 & 2.4405 \\
\hline
\end{tabular}




\begin{tabular}{|c|c|c|c|c|c|c|c|c|c|c|c|c|c|c|}
\hline \multicolumn{15}{|c|}{ Fibre No: } \\
\hline & 57 & 58 & 59 & 60 & 61 & 62 & 63 & 64 & 65 & 66 & 67 & 68 & 69 & 70 \\
\hline $\mathrm{X}$ & -2.0581 & -2.0760 & -0.2569 & -1.7449 & -1.5098 & -1.9112 & 1.7728 & 2.0323 & 1.3204 & 0.6453 & 0.9603 & 1.6784 & 2.3611 & 1.2917 \\
\hline $\mathrm{y}$ & -0.3457 & -0.3948 & -0.5522 & -1.8844 & -0.5832 & -1.3212 & 0.3957 & 1.3952 & 0.7815 & 0.9348 & 0.7459 & 1.6958 & 1.1533 & 1.6601 \\
\hline $\mathrm{Z}$ & -1.0086 & -2.4725 & -1.5975 & -1.6756 & -0.8190 & -1.3909 & 0.4472 & 1.8516 & 1.4792 & 0.9924 & 0.2090 & 1.2888 & 2.3536 & 1.0086 \\
\hline d & 0.5050 & 0.5726 & 0.5175 & 0.5527 & 0.4890 & 0.5379 & 0.3648 & 0.5201 & 0.4843 & 0.4774 & 0.4232 & 0.5225 & 0.5235 & 0.5043 \\
\hline$\underline{t}$ & 1.7688 & 2.3360 & 7.9477 & 0.5644 & 1.1744 & 3.2967 & 8.6023 & 1.3533 & 4.3085 & 4.7590 & 2.5392 & 7.2077 & 4.6043 & 9.8650 \\
\hline \multicolumn{15}{|c|}{ Fibre No: } \\
\hline & 71 & 72 & 73 & 74 & 75 & & & & & & & & & \\
\hline $\mathrm{X}$ & 0.3740 & 2.0829 & 2.2938 & 1.7339 & 1.7665 & & & & & & & & & \\
\hline$y$ & 1.6826 & 0.0394 & 0.6676 & 1.1422 & 1.7750 & & & & & & & & & \\
\hline z & 0.8633 & 0.7235 & 2.4292 & 2.0478 & 1.0950 & & & & & & & & & \\
\hline d & 0.5010 & 0.4182 & 0.5312 & 0.5570 & 0.5037 & & & & & & & & & \\
\hline $\mathrm{t}$ & 5.2281 & 5.4614 & 8.4398 & 1.6075 & 7.5171 & & & & & & & & & \\
\hline
\end{tabular}

Table A.4.14 Myopathy MUAP - reinnervation of 25fibres in both planes of the MU, results after 5294 iterations. 


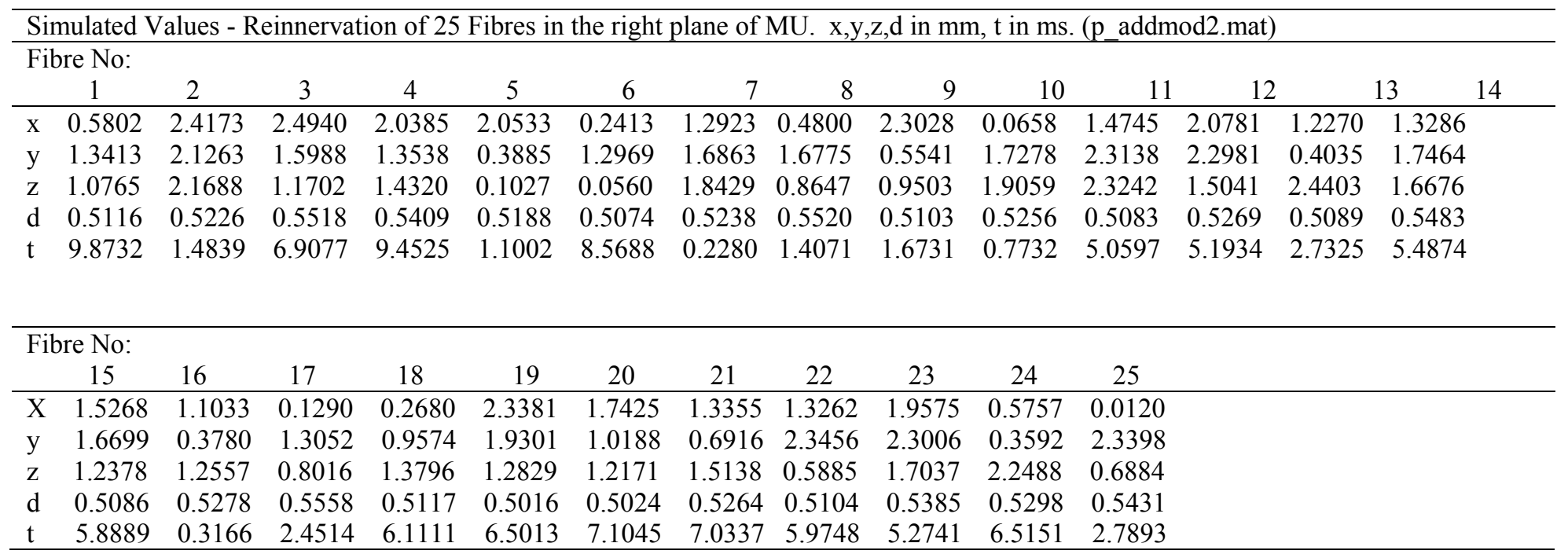

Table A4.15. Myopathic MUAP - reinnervation of 25 fibres in the right plane of the MU, simulated values. 


\begin{tabular}{|c|c|c|c|c|c|c|c|c|c|c|c|c|c|c|}
\hline \multicolumn{15}{|c|}{ Initial values $\mathrm{x}, \mathrm{y}, \mathrm{z}, \mathrm{d}$ in $\mathrm{mm}, \mathrm{t}$ in $\mathrm{ms}$ (zren2.mat) } \\
\hline \multicolumn{15}{|c|}{ Fibre No: } \\
\hline 1 & 1 & 2 & 3 & 4 & 5 & 6 & 7 & 8 & 9 & 10 & 11 & 12 & 13 & 14 \\
\hline $\mathrm{x}$ & -0.5474 & -0.9588 & -1.3243 & -1.0437 & -1.3173 & -2.2758 & -0.8206 & -0.6176 & -0.1817 & -1.9162 & -0.4163 & -2.2616 & -1.2349 & -1.2518 \\
\hline $\mathrm{y}$ & -0.1176 & -1.2985 & -1.6779 & -1.7169 & -0.2299 & -1.9055 & -1.5816 & -2.4564 & -1.5791 & -1.1943 & -1.2163 & -1.2613 & -0.6654 & -0.9604 \\
\hline z & -1.6972 & -2.0774 & -0.0192 & -1.4724 & -1.6348 & -0.6561 & -1.8910 & -1.8067 & -2.2118 & -0.5944 & -2.2441 & -1.2907 & -0.2268 & -0.6927 \\
\hline $\mathrm{d}$ & 0.5215 & 0.5559 & 0.5140 & 0.5517 & 0.5101 & 0.5543 & 0.5589 & 0.5304 & 0.5273 & 0.5270 & 0.5491 & 0.5217 & 0.5537 & 0.5497 \\
\hline $\mathrm{t}$ & 9.3469 & 0.5346 & 0.6684 & 8.4617 & 7.0119 & 7.3608 & 3.6534 & 6.5152 & 4.3641 & 3.5926 & 0.6056 & 9.8664 & 0.7375 & 5.2975 \\
\hline
\end{tabular}
Fibre No:

\begin{tabular}{lllllllllllllllllll}
\multicolumn{1}{c}{15} & 16 & 17 & 18 & 19 & 20 & 21 & 22 & 23 & 24 & 25 & 26 & 27 & 28 \\
\hline $\mathrm{x}$ & -1.1611 & -2.0695 & -1.5739 & -0.5830 & -2.1150 & -1.3433 & -1.4291 & -2.3884 & -2.1051 & -0.2275 & -1.7745 & 2.2175 & 0.9693 & 1.4753 \\
$\mathrm{y}$ & -2.3524 & -0.3134 & -1.8406 & -0.7658 & -1.0302 & -1.1698 & -2.0060 & -1.8707 & -0.3994 & -0.6865 & -2.3447 & 1.6301 & 1.2494 & 2.3885 \\
$\mathrm{z}$ & -0.1252 & -0.0397 & -1.8135 & -0.8775 & -2.1038 & -0.7180 & -0.0826 & -1.3865 & -0.5319 & -0.0075 & -0.5998 & 0.3758 & 0.3688 & 1.3904 \\
$\mathrm{~d}$ & 0.5314 & 0.5226 & 0.5599 & 0.5016 & 0.5277 & 0.5386 & 0.5041 & 0.5469 & 0.5258 & 0.5103 & 0.5383 & 0.5218 & 0.5105 & 0.5422 \\
$\mathrm{t}$ & 7.7020 & 8.6825 & 8.8857 & 5.9111 & 4.1539 & 1.5372 & 4.9848 & 6.2485 & 1.3043 & 0.2688 & 3.1754 & 3.8581 & 8.4558 & 9.8331 \\
\hline
\end{tabular}
Fibre No:

\begin{tabular}{lllllllllllllllll}
\multicolumn{2}{c}{29} & 30 & 31 & 32 & 33 & 34 & 35 & 36 & 37 & 38 & 39 & 40 & 41 & 42 \\
\hline $\mathrm{x}$ & 1.0219 & 1.1601 & 1.5732 & 0.6196 & 2.2542 & 0.3280 & 0.9133 & 0.8738 & 0.5381 & 1.1782 & 1.1556 & 1.7225 & 0.7233 & 1.4418 \\
$\mathrm{y}$ & 0.3545 & 2.4027 & 0.3168 & 1.1911 & 1.0662 & 2.2141 & 0.6326 & 1.1308 & 1.6990 & 1.2649 & 2.3784 & 1.7555 & 1.3436 & 2.1914 \\
$\mathrm{z}$ & 1.4122 & 0.3151 & 1.6281 & 0.9733 & 0.3551 & 0.2304 & 0.3378 & 2.0224 & 2.2723 & 1.5010 & 1.5818 & 2.4679 & 1.2861 & 1.1001 \\
$\mathrm{~d}$ & 0.5297 & 0.5360 & 0.5146 & 0.5356 & 0.5537 & 0.5405 & 0.5340 & 0.5518 & 0.5300 & 0.5381 & 0.5073 & 0.5545 & 0.5476 & 0.5276 \\
$\mathrm{t}$ & 4.8851 & 3.1925 & 8.0307 & 0.2838 & 4.1031 & 0.7106 & 4.5531 & 6.5165 & 8.6086 & 7.5584 & 8.2470 & 8.5127 & 4.1403 & 8.6926 \\
\hline
\end{tabular}
Fibre No:

\begin{tabular}{lllllllllllllllllll}
\multicolumn{1}{l}{43} & 44 & 45 & 46 & 47 & 48 & 49 & 50 & 51 & 52 & 53 & 54 & 55 & 56 \\
\hline $\mathrm{x}$ & 1.7891 & 2.2151 & 1.2224 & 2.1647 & 2.4734 & 2.2038 & 0.5288 & 0.0015 & 1.0443 & 1.7723 & 1.5739 & 2.3804 & 0.7101 & 0.4849 \\
$\mathrm{y}$ & 2.0018 & 1.3125 & 1.6692 & 2.2250 & 0.5388 & 1.0993 & 2.4978 & 0.0022 & 1.7062 & 2.0718 & 0.5346 & 2.3689 & 1.9422 & 0.0283 \\
$\mathrm{z}$ & 1.7663 & 1.1583 & 1.7051 & 1.3599 & 1.1151 & 1.1688 & 0.3840 & 1.9334 & 1.7014 & 0.2364 & 0.5339 & 0.9746 & 1.9597 & 0.4796 \\
$\mathrm{~d}$ & 0.5290 & 0.5522 & 0.5361 & 0.5433 & 0.5221 & 0.5368 & 0.5157 & 0.5273 & 0.5354 & 0.5502 & 0.5503 & 0.5277 & 0.5093 & 0.5580 \\
$\mathrm{t}$ & 0.1909 & 7.1342 & 9.1663 & 4.5035 & 5.1466 & 3.6516 & 6.1635 & 3.1918 & 8.3642 & 7.6400 & 3.8882 & 6.9217 & 2.8216 & 2.4405
\end{tabular}




\begin{tabular}{|c|c|c|c|c|c|c|c|c|c|c|c|c|c|c|}
\hline \multicolumn{15}{|c|}{ Fibre No: } \\
\hline & 7 & 58 & 59 & 60 & 61 & 62 & 63 & 64 & 65 & 66 & 67 & 68 & 69 & 70 \\
\hline$x$ & 2.0493 & 2.0709 & 0.2541 & 1.7406 & 1.4955 & 1.9064 & 1.7575 & 2.0332 & 1.3214 & 0.6471 & 0.9696 & 1.6795 & 2.3619 & 1.2929 \\
\hline $\mathrm{y}$ & 0.3411 & 0.3943 & 0.5485 & 1.8824 & 0.5675 & 1.3153 & 0.3576 & $5 \quad 1.3921$ & 0.7768 & 0.9256 & 0.6982 & 1.6906 & 1.1517 & 1.6534 \\
\hline Z & 0.9954 & 2.4698 & 1.5868 & 1.6738 & 0.7969 & 1.3848 & 0.4042 & 1.8475 & 1.4703 & 0.9825 & 0.1957 & 1.2848 & 2.3504 & 1.0046 \\
\hline d & 0.5121 & 0.5291 & 0.5235 & 0.5160 & 0.5240 & 0.5106 & 0.5018 & 0.5221 & 0.5022 & 0.5064 & 0.5156 & 0.5274 & 0.5214 & 0.5127 \\
\hline$\underline{t}$ & 1.7688 & 2.3360 & 7.9477 & 0.5644 & 1.1744 & 3.2967 & 8.6023 & 1.3533 & 4.3085 & 4.7590 & 2.5392 & 7.2077 & 4.6043 & 9.8650 \\
\hline \multicolumn{15}{|c|}{ Fibre No: } \\
\hline \multicolumn{2}{|c|}{71} & 72 & 73 & 74 & 75 & & & & & & & & & \\
\hline $\mathrm{X}$ & 0.3760 & 2.0731 & 2.2946 & 1.7351 & 1.7674 & & & & & & & & & \\
\hline$y$ & 1.6752 & 0.0374 & 0.6665 & 1.1394 & 1.7696 & & & & & & & & & \\
\hline$z$ & 0.8595 & 0.6855 & 2.4252 & 2.0428 & 1.0916 & & & & & & & & & \\
\hline d & 0.5050 & 0.5172 & 0.5304 & 0.5573 & 0.5099 & & & & & & & & & \\
\hline $\mathrm{t}$ & 5.2281 & 5.4614 & 8.4398 & 1.6075 & 7.5171 & & & & & & & & & \\
\hline
\end{tabular}

Table A.4.16 Myopathy MUAP - reinnervation of 25 fibres in the right plane of the MU, initial values. 


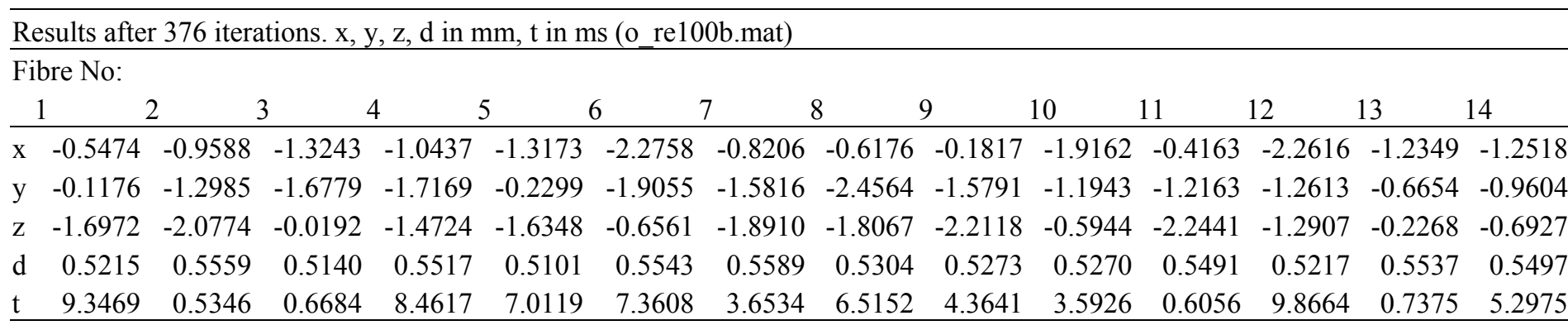
Fibre No:

\begin{tabular}{|c|c|c|c|c|c|c|c|c|c|c|c|c|c|c|}
\hline 1 & 5 & 16 & 17 & 18 & 19 & 20 & 21 & 22 & 23 & 24 & 25 & 26 & 27 & 28 \\
\hline $\mathrm{x}$ & -1.1611 & -2.0695 & -1.5739 & -0.5830 & -2.1150 & -1.3433 & -1.4291 & -2.3884 & -2.1051 & -0.2275 & -1.7745 & 2.2175 & 0.9693 & 1.4753 \\
\hline y & -2.3524 & -0.3134 & -1.8406 & -0.7658 & -1.0302 & -1.1698 & $8-2.0060$ & -1.8707 & -0.3994 & -0.6865 & -2.3447 & 01 & 1.2494 & 85 \\
\hline z & -0.1252 & -0.0397 & -1.8135 & -0.8775 & -2.1038 & -0.7180 & $0 \quad-0.0826$ & -1.3865 & -0.5319 & -0.0075 & -0.5998 & 758 & 0.3688 & 1.3904 \\
\hline d & 0.5314 & 226 & 0.5599 & 0.5016 & 277 & 386 & 5041 & 169 & 258 & 03 & 0.5383 & 218 & 0.5105 & 0.5422 \\
\hline & 7.7020 & 8.6825 & 8.8857 & 5.9111 & 4.1539 & 1.5372 & $2 \quad 4.9848$ & 6.2485 & 1.3043 & 0.2688 & 3.1754 & 3.8581 & 8.4558 & 9.8331 \\
\hline
\end{tabular}
Fibre No:

\begin{tabular}{lllllllllllllllllll}
\multicolumn{2}{c}{29} & 30 & 31 & 32 & 33 & 34 & 35 & 36 & 37 & 38 & 39 & 40 & 41 & 42 \\
\hline $\mathrm{x}$ & 1.0219 & 1.1601 & 1.5732 & 0.6196 & 2.2542 & 0.3280 & 0.9133 & 0.8738 & 0.5381 & 1.1782 & 1.1556 & 1.7225 & 0.7233 & 1.4418 \\
$\mathrm{y}$ & 0.3545 & 2.4027 & 0.3168 & 1.1911 & 1.0662 & 2.2141 & 0.6326 & 1.1308 & 1.6990 & 1.2649 & 2.3784 & 1.7555 & 1.3436 & 2.1914 \\
$\mathrm{z}$ & 1.4122 & 0.3151 & 1.6281 & 0.9733 & 0.3551 & 0.2304 & 0.3378 & 2.0224 & 2.2723 & 1.5010 & 1.5818 & 2.4679 & 1.2861 & 1.1001 \\
$\mathrm{~d}$ & 0.5297 & 0.5360 & 0.5146 & 0.5356 & 0.5537 & 0.5405 & 0.5340 & 0.5518 & 0.5300 & 0.5381 & 0.5073 & 0.5545 & 0.5476 & 0.5276 \\
$\mathrm{t}$ & 4.8851 & 3.1925 & 8.0307 & 0.2838 & 4.1031 & 0.7106 & 4.5531 & 6.5165 & 8.6086 & 7.5584 & 8.2470 & 8.5127 & 4.1403 & 8.6926 \\
\hline
\end{tabular}
Fibre No:

\begin{tabular}{llllllllllllllllllll}
\multicolumn{1}{l}{43} & 44 & 45 & 46 & 47 & 48 & 49 & 50 & 51 & 52 & 53 & 54 & 55 & 56 \\
$\mathrm{x}$ & 1.7891 & 2.2151 & 1.2224 & 2.1647 & 2.4734 & 2.2038 & 0.5288 & 0.0015 & 1.0443 & 1.7723 & 1.5739 & 2.3804 & 0.7101 & 0.4849 \\
$\mathrm{y}$ & 2.0018 & 1.3125 & 1.6692 & 2.2250 & 0.5388 & 1.0993 & 2.4978 & 0.0022 & 1.7062 & 2.0718 & 0.5346 & 2.3689 & 1.9422 & 0.0283 \\
$\mathrm{z}$ & 1.7663 & 1.1583 & 1.7051 & 1.3599 & 1.1151 & 1.1688 & 0.3840 & 1.9334 & 1.7014 & 0.2364 & 0.5339 & 0.9746 & 1.9597 & 0.4796 \\
$\mathrm{~d}$ & 0.5290 & 0.5522 & 0.5361 & 0.5433 & 0.5221 & 0.5368 & 0.5157 & 0.5273 & 0.5354 & 0.5502 & 0.5503 & 0.5277 & 0.5093 & 0.5580 \\
$\mathrm{t}$ & 0.1909 & 7.1342 & 9.1663 & 4.5035 & 5.1466 & 3.6516 & 6.1635 & 3.1918 & 8.3642 & 7.6400 & 3.8882 & 6.9217 & 2.8216 & 2.4405
\end{tabular}




\begin{tabular}{|c|c|c|c|c|c|c|c|c|c|c|c|c|c|c|}
\hline \multicolumn{15}{|c|}{ Fibre No: } \\
\hline & 7 & 58 & 59 & 60 & 61 & 62 & 63 & 64 & 65 & 66 & 67 & 68 & 69 & 70 \\
\hline $\mathrm{x}$ & 2.0493 & 2.0709 & 0.2541 & 1.7406 & 1.4955 & 1.9064 & 1.7575 & 2.0332 & 1.3214 & 0.6471 & 0.9696 & 1.6795 & 2.3619 & 1.2929 \\
\hline $\mathrm{y}$ & 0.3411 & 0.3943 & 0.5485 & 1.8824 & 0.5675 & 1.3153 & 0.3576 & 1.3921 & 0.7768 & 0.9256 & 0.6982 & 1.6906 & 1.1517 & 1.6534 \\
\hline Z & 0.9954 & 2.4698 & 1.5868 & 1.6738 & 0.7969 & 1.3848 & 0.4042 & 1.8475 & 1.4703 & 0.9825 & 0.1957 & 1.2848 & 2.3504 & 1.0046 \\
\hline d & 0.5121 & 0.5291 & 0.5235 & 0.5160 & 0.5240 & 0.5106 & 0.5018 & 0.5221 & 0.5022 & 0.5064 & 0.5156 & 0.5274 & 0.5214 & 0.5127 \\
\hline$\underline{t}$ & 1.7688 & 2.3360 & 7.9477 & 0.5644 & 1.1744 & 3.2967 & 8.6023 & 1.3533 & 4.3085 & 4.7590 & 2.5392 & 7.2077 & 4.6043 & 9.8650 \\
\hline \multicolumn{15}{|c|}{ Fibre No: } \\
\hline \multicolumn{2}{|c|}{71} & 72 & 73 & 74 & 75 & & & & & & & & & \\
\hline X & 0.3760 & 2.0731 & 2.2946 & 1.7351 & 1.7674 & & & & & & & & & \\
\hline$y$ & 1.6752 & 0.0374 & 0.6665 & 1.1394 & 1.7696 & & & & & & & & & \\
\hline$z$ & 0.8595 & 0.6855 & 2.4252 & 2.0428 & 1.0916 & & & & & & & & & \\
\hline d & 0.5050 & 0.5172 & 0.5304 & 0.5573 & 0.5099 & & & & & & & & & \\
\hline $\mathrm{t}$ & 5.2281 & 5.4614 & 8.4398 & 1.6075 & 7.5171 & & & & & & & & & \\
\hline
\end{tabular}

Table A.4.17 Myopathy MUAP - reinnervation of 25 fibres in the right plane of the MU, results after 376 iterations. 


\begin{tabular}{|c|c|c|c|c|c|c|c|c|c|c|c|c|c|c|}
\hline \multicolumn{15}{|c|}{ esults after 1133 iterations. $\mathrm{x}, \mathrm{y}, \mathrm{z}, \mathrm{d}$ in $\mathrm{mm}, \mathrm{t}$ in $\mathrm{ms}$ (o_r1000b.mat) } \\
\hline \multicolumn{15}{|c|}{ bre No: } \\
\hline & L & 2 & 3 & 4 & 5 & 6 & 7 & 8 & 9 & 10 & 11 & 12 & 13 & 14 \\
\hline A & -0.5520 & -0.9613 & -1.3294 & -1.0469 & -1.3225 & -2.2803 & -0.8231 & -0.6188 & -0.1833 & -1.9238 & -0.4184 & -2.2669 & -1.2356 & -1.26 \\
\hline y & -0.1178 & -1.2977 & -1.6763 & -1.7156 & -0.2297 & -1.8985 & -1.5807 & -2.4555 & -1.5789 & -1.1920 & -1.2161 & -1.2565 & -0.6353 & -0.95 \\
\hline Z & -1.6994 & -2.0760 & -0.0192 & -1.4713 & -1.6334 & -0.6537 & -1.8900 & -1.8060 & -2.2115 & -0.5933 & -2.2437 & -1.2858 & -0.2166 & -0.6922 \\
\hline$d$ & 0.5327 & 0.5733 & 0.5397 & 0.5710 & 0.5356 & 0.5947 & 0.5751 & 0.5419 & 0.5373 & 0.5714 & 0.5614 & 0.5650 & 0.5972 & 0.576 \\
\hline & 9.3469 & 0.5346 & 0.6684 & 8.4617 & 7.0119 & 7.3608 & 3.6534 & 6.5152 & 4.3641 & 3.5926 & 0.6056 & 9.8664 & 0.7375 & 5.2975 \\
\hline
\end{tabular}
Fibre No:

\begin{tabular}{|c|c|c|c|c|c|c|c|c|c|c|c|c|c|c|}
\hline & 15 & 16 & 17 & 18 & 19 & 20 & 21 & 22 & 23 & 24 & 25 & 26 & 27 & 28 \\
\hline $\mathbf{y}$ & -1.1638 & -2.0728 & -1.5762 & -0.5914 & -2.1177 & -1.3505 & -1.4327 & -2.391 & -2.1009 & -0.257 & -1.7772 & 2.2173 & 0.9670 & 1.4743 \\
\hline & -2.3504 & -0.3132 & -1.8382 & -0.7692 & -1.0280 & -1.1690 & -2.0035 & -1.8655 & -0.4168 & -0.7230 & -2.3410 & 71 & 46 & 97 \\
\hline & -0.1251 & -0.0396 & -1.8112 & -0.8814 & -2.0993 & -0.7175 & -0.0825 & -1.3826 & -0.5550 & -0.0079 & -0.5988 & 0.3774 & 0.3733 & 1.3911 \\
\hline $\mathrm{a}$ & 0.5506 & 160 & 18 & 5048 & 568 & 674 & 285 & 03 & 569 & 4606 & 0.5633 & 63 & 40 & 0.5444 \\
\hline & 7.7020 & 8.6825 & 8.8857 & 5.9111 & 4.1539 & 1.5372 & 4.9848 & 6.2485 & 1.3043 & 0.2688 & 3.1754 & 3.8581 & 8.4558 & 9.8331 \\
\hline
\end{tabular}
Fibre No:

\begin{tabular}{llllllllllllllllll}
\multicolumn{2}{c}{29} & 30 & 31 & 32 & 33 & 34 & 35 & 36 & 37 & 38 & 39 & 40 & 41 & 42 \\
\hline $\mathrm{x}$ & 1.0197 & 1.1587 & 1.5722 & 0.6162 & 2.2569 & 0.3257 & 0.9180 & 0.8721 & 0.5369 & 1.1764 & 1.1547 & 1.7216 & 0.7207 & 1.4407 \\
$\mathrm{y}$ & 0.3576 & 2.4050 & 0.3183 & 1.1990 & 1.0814 & 2.2162 & 0.6950 & 1.1320 & 1.6992 & 1.2682 & 2.3791 & 1.7561 & 1.3478 & 2.1934 \\
$\mathrm{z}$ & 1.4244 & 0.3154 & 1.6360 & 0.9798 & 0.3601 & 0.2307 & 0.3711 & 2.0246 & 2.2726 & 1.5050 & 1.5823 & 2.4686 & 1.2901 & 1.1011 \\
$\mathrm{~d}$ & 0.5104 & 0.5373 & 0.5003 & 0.5240 & 0.5141 & 0.5457 & 0.4244 & 0.5538 & 0.5362 & 0.5332 & 0.5108 & 0.5571 & 0.5441 & 0.5278 \\
$\mathrm{t}$ & 4.8851 & 3.1925 & 8.0307 & 0.2838 & 4.1031 & 0.7106 & 4.5531 & 6.5165 & 8.6086 & 7.5584 & 8.2470 & 8.5127 & 4.1403 & 8.6926 \\
\hline
\end{tabular}

\section{Fibre No:}

\begin{tabular}{llllllllllllllllllll}
\multicolumn{1}{c}{43} & 44 & 45 & 46 & 47 & 48 & 49 & 50 & 51 & 52 & 53 & 54 & 55 & 56 \\
$\mathrm{x}$ & 1.7883 & 2.2147 & 1.2210 & 2.1640 & 2.4751 & 2.2037 & 0.5273 & -0.0017 & 1.0429 & 1.7712 & 1.5830 & 2.3798 & 0.7089 & 0.4965 \\
$\mathrm{y}$ & 2.0031 & 1.3178 & 1.6709 & 2.2268 & 0.5436 & 1.1050 & 2.4989 & 0.0022 & 1.7078 & 2.0763 & 0.5641 & 2.3708 & 1.9426 & 0.0412 \\
$\mathrm{z}$ & 1.7675 & 1.1630 & 1.7069 & 1.3610 & 1.1250 & 1.1749 & 0.3842 & 1.9363 & 1.7030 & 0.2369 & 0.5633 & 0.9754 & 1.9602 & 0.6984 \\
$\mathrm{~d}$ & 0.5295 & 0.5379 & 0.5367 & 0.5422 & 0.4921 & 0.5188 & 0.5209 & 0.5328 & 0.5371 & 0.5443 & 0.4633 & 0.5258 & 0.5144 & 0.3213 \\
$\mathrm{t}$ & 0.1909 & 7.1342 & 9.1663 & 4.5035 & 5.1466 & 3.6516 & 6.1635 & 3.1918 & 8.3642 & 7.6400 & 3.8882 & 6.9217 & 2.8216 & 2.4405
\end{tabular}




\begin{tabular}{|c|c|c|c|c|c|c|c|c|c|c|c|c|c|c|}
\hline \multicolumn{15}{|c|}{ Fibre No: } \\
\hline & 7 & 58 & 59 & 60 & 61 & 62 & 63 & 64 & 65 & 66 & 67 & 68 & 69 & 70 \\
\hline $\mathrm{X}$ & 2.0521 & 2.0701 & 0.2505 & 1.7397 & 1.4981 & 1.9057 & 1.7729 & 2.0324 & 1.3200 & 0.6436 & 0.9745 & 1.6784 & 2.3613 & 1.2914 \\
\hline $\mathrm{y}$ & 0.3466 & 0.3947 & 0.5506 & 1.8839 & 0.5836 & 1.3188 & 0.3812 & 1.3939 & 0.7805 & 0.9349 & 0.7584 & 1.6938 & 1.1526 & 1.6577 \\
\hline $\mathrm{Z}$ & 1.0114 & 2.4721 & 1.5927 & 1.6752 & 0.8195 & 1.3885 & 0.4308 & 1.8498 & 1.4771 & 0.9925 & 0.2125 & 1.2873 & 2.3521 & 1.0072 \\
\hline d & 0.4692 & 0.5275 & 0.5214 & 0.5156 & 0.4678 & 0.5016 & 0.3992 & 0.5187 & 0.4895 & 0.4866 & 0.4126 & 0.5228 & 0.5200 & 0.5068 \\
\hline$\underline{t}$ & 1.7688 & 2.3360 & 7.9477 & 0.5644 & 1.1744 & 3.2967 & 8.6023 & 1.3533 & 4.3085 & 4.7590 & 2.5392 & 7.2077 & 4.6043 & 9.8650 \\
\hline \multicolumn{15}{|c|}{ Fibre No: } \\
\hline \multicolumn{2}{|c|}{71} & 72 & 73 & 74 & 75 & & & & & & & & & \\
\hline X & 0.3734 & 2.0824 & 2.2940 & 1.7340 & 1.7665 & & & & & & & & & \\
\hline$y$ & 1.6789 & 0.0387 & 0.6671 & 1.1409 & 1.7730 & & & & & & & & & \\
\hline$z$ & 0.8614 & 0.7100 & 2.4274 & 2.0455 & 1.0937 & & & & & & & & & \\
\hline d & 0.5055 & 0.4441 & 0.5283 & 0.5551 & 0.5044 & & & & & & & & & \\
\hline $\mathrm{t}$ & 5.2281 & 5.4614 & 8.4398 & 1.6075 & 7.5171 & & & & & & & & & \\
\hline
\end{tabular}

Table A.4.18 Myopathy MUAP - reinnervation of 25 fibres in the right plane of the MU, results after 1133 iterations. 


\begin{tabular}{|c|c|c|c|c|c|c|c|c|c|c|c|c|c|c|}
\hline \multicolumn{15}{|c|}{ esults after 5295 iterations. $\mathrm{x}, \mathrm{y}, \mathrm{z}, \mathrm{d}$ in $\mathrm{mm}, \mathrm{t}$ in $\mathrm{ms}$ (o_r5000b.mat) } \\
\hline \multicolumn{15}{|c|}{ bre No: } \\
\hline & L & 2 & 3 & 4 & 5 & 6 & 7 & 8 & 9 & 10 & 11 & 12 & 13 & 14 \\
\hline & -0.5502 & -0.9619 & -1.3253 & -1.0465 & -1.3183 & -2.2769 & -0.8236 & -0.6203 & -0.1841 & -1.9140 & -0.4190 & -2.2598 & -1.1990 & -1.25 \\
\hline $\mathrm{y}$ & -0.1180 & -1.3017 & -1.6830 & -1.7222 & -0.2306 & -1.9220 & -1.5852 & -2.4580 & -1.5811 & -1.1930 & -1.2185 & -1.2717 & -0.6687 & -0.95 \\
\hline $\mathrm{z}$ & -1.7032 & -2.0824 & -0.0193 & -1.4769 & -1.6395 & -0.6618 & -1.8954 & -1.8078 & -2.2146 & -0.5938 & -2.2483 & -1.3014 & -0.2280 & -0.6899 \\
\hline 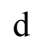 & 0.5319 & 0.5887 & 0.5302 & 0.5812 & 0.5257 & 0.5862 & 0.5907 & 0.5656 & 0.5547 & 0.5407 & 0.5776 & 0.5412 & 0.5861 & 0.564 \\
\hline & 9.3469 & 0.5346 & 0.6684 & 8.4617 & 7.0119 & 7.3608 & 3.6534 & 6.5152 & 4.3641 & 3.5926 & 0.6056 & 9.8664 & 0.7375 & 5.2975 \\
\hline
\end{tabular}
Fibre No:

\begin{tabular}{|c|c|c|c|c|c|c|c|c|c|c|c|c|c|c|}
\hline & 5 & 16 & 17 & 18 & 19 & 20 & 21 & 22 & 23 & 24 & 25 & 26 & 27 & 28 \\
\hline $\mathrm{x}$ & -1.1639 & -2.0690 & -1.5779 & -0.5896 & -2.1186 & -1.3445 & -1.4307 & -2.3929 & -2.1139 & -0.2824 & -1.7781 & 2.2176 & 0.9667 & 1.4738 \\
\hline $\mathrm{y}$ & -2.3587 & -0.3157 & -1.8450 & -0.7653 & -1.0348 & -1.1698 & -2.0133 & -1.8818 & -0.4162 & -0.6752 & -2.3525 & $5 \quad 1.6399$ & 1.2685 & 2.3915 \\
\hline $\mathrm{z}$ & -0.1255 & -0.0400 & -1.8179 & -0.8770 & -2.1131 & -0.7180 & -0.0829 & -1.3946 & -0.5543 & -0.0074 & -0.6018 & $3 \quad 0.3781$ & 0.3745 & 1.3921 \\
\hline d & 0.5631 & 0.5309 & 0.6037 & 0.5040 & 0.5704 & 0.5525 & 0.5283 & 0.5948 & 0.5429 & 0.4940 & 0.5794 & $4 \quad 0.5095$ & 0.4814 & 0.5587 \\
\hline $\mathrm{t}$ & 7.7020 & 8.6825 & 8.8857 & 5.9111 & 4.1539 & 1.5372 & 4.9848 & 6.2485 & 1.3043 & 0.2688 & 3.1754 & $4 \quad 3.8581$ & 8.4558 & 9.8331 \\
\hline
\end{tabular}
Fibre No:

\begin{tabular}{llllllllllllllllll}
\multicolumn{2}{r}{29} & 30 & 31 & 32 & 33 & 34 & 35 & 36 & 37 & 38 & 39 & 40 & 41 & 42 \\
\hline $\mathrm{x}$ & 1.0196 & 1.1585 & 1.5723 & 0.6163 & 2.2579 & 0.3257 & 0.9170 & 0.8719 & 0.5362 & 1.1765 & 1.1542 & 1.7210 & 0.7209 & 1.4404 \\
$\mathrm{y}$ & 0.3585 & 2.4077 & 0.3189 & 1.2015 & 1.0863 & 2.2201 & 0.7089 & 1.1336 & 1.7008 & 1.2703 & 2.3807 & 1.7570 & 1.3504 & 2.1956 \\
$\mathrm{z}$ & 1.4281 & 0.3157 & 1.6391 & 0.9818 & 0.3617 & 0.2311 & 0.3785 & 2.0275 & 2.2747 & 1.5074 & 1.5834 & 2.4700 & 1.2926 & 1.1022 \\
$\mathrm{~d}$ & 0.5101 & 0.5488 & 0.5029 & 0.5248 & 0.5094 & 0.5555 & 0.4048 & 0.5647 & 0.5530 & 0.5396 & 0.5255 & 0.5730 & 0.5491 & 0.5391 \\
$\mathrm{t}$ & 4.8851 & 3.1925 & 8.0307 & 0.2838 & 4.1031 & 0.7106 & 4.5531 & 6.5165 & 8.6086 & 7.5584 & 8.2470 & 8.5127 & 4.1403 & 8.6926 \\
\hline
\end{tabular}
Fibre No:

\begin{tabular}{lllllllllllllllllll}
\multicolumn{1}{l}{43} & 44 & 45 & 46 & 47 & 48 & 49 & 50 & 51 & 52 & 53 & 54 & 55 & 56 \\
\hline $\mathrm{x}$ & 1.7879 & 2.2150 & 1.2208 & 2.1637 & 2.4759 & 2.2040 & 0.5269 & -0.0011 & 1.0426 & 1.7712 & 1.5850 & 2.3795 & 0.7084 & 0.4920 \\
$\mathrm{y}$ & 2.0045 & 1.3201 & 1.6728 & 2.2284 & 0.5453 & 1.1072 & 2.5019 & 0.0022 & 1.7098 & 2.0791 & 0.5720 & 2.3724 & 1.9443 & 0.0421 \\
$\mathrm{z}$ & 1.7687 & 1.1651 & 1.7088 & 1.3619 & 1.1284 & 1.1772 & 0.3846 & 1.9404 & 1.7050 & 0.2372 & 0.5712 & 0.9761 & 1.9619 & 0.7129 \\
$\mathrm{~d}$ & 0.5422 & 0.5423 & 0.5478 & 0.5543 & 0.4901 & 0.5214 & 0.5340 & 0.5377 & 0.5486 & 0.5523 & 0.4453 & 0.5370 & 0.5293 & 0.3050 \\
$\mathrm{t}$ & 0.1909 & 7.1342 & 9.1663 & 4.5035 & 5.1466 & 3.6516 & 6.1635 & 3.1918 & 8.3642 & 7.6400 & 3.8882 & 6.9217 & 2.8216 & 2.4405
\end{tabular}




\begin{tabular}{|c|c|c|c|c|c|c|c|c|c|c|c|c|c|c|}
\hline \multicolumn{15}{|c|}{ Fibre No: } \\
\hline & 7 & 58 & 59 & 60 & 61 & 62 & 63 & 64 & 65 & 66 & 67 & 68 & 69 & 70 \\
\hline $\mathrm{X}$ & 2.0531 & 2.0699 & 0.2509 & 1.7395 & 1.4986 & 1.9057 & 1.7765 & 2.0323 & 1.3201 & 0.6433 & 0.9739 & 1.6784 & 2.3610 & 1.2915 \\
\hline $\mathrm{y}$ & 0.3483 & 0.3950 & 0.5515 & 1.8854 & 0.5881 & 1.3206 & 0.3870 & 1.3951 & 0.7819 & 0.9370 & 0.7724 & 1.6958 & 1.1533 & 1.6601 \\
\hline Z & 1.0163 & 2.4740 & 1.5955 & 1.6765 & 0.8258 & 1.3904 & 0.4375 & 1.8515 & 1.4798 & 0.9947 & 0.2164 & 1.2888 & 2.3536 & 1.0087 \\
\hline d & 0.4626 & 0.5386 & 0.5234 & 0.5269 & 0.4576 & 0.5071 & 0.3764 & 0.5282 & 0.4920 & 0.4852 & 0.3934 & 0.5308 & 0.5314 & 0.5125 \\
\hline$\underline{t}$ & 1.7688 & 2.3360 & 7.9477 & 0.5644 & 1.1744 & 3.2967 & 8.6023 & 1.3533 & 4.3085 & 4.7590 & 2.5392 & 7.2077 & 4.6043 & 9.8650 \\
\hline \multicolumn{15}{|c|}{ Fibre No: } \\
\hline \multicolumn{2}{|c|}{71} & 72 & 73 & 74 & 75 & & & & & & & & & \\
\hline X & 0.3737 & 2.0849 & 2.2937 & 1.7338 & 1.7664 & & & & & & & & & \\
\hline$y$ & 1.6817 & 0.0391 & 0.6676 & 1.1422 & 1.7750 & & & & & & & & & \\
\hline$z$ & 0.8629 & 0.7169 & 2.4292 & 2.0478 & 1.0950 & & & & & & & & & \\
\hline d & 0.5101 & 0.4288 & 0.5393 & 0.5657 & 0.5117 & & & & & & & & & \\
\hline $\mathrm{t}$ & 5.2281 & 5.4614 & 8.4398 & 1.6075 & 7.5171 & & & & & & & & & \\
\hline
\end{tabular}

Table A.4.19 Myopathy MUAP - reinnervation of 25 fibres in the right plane of the MU, results after 5295 iterations. 